US Army Corps

of Engineers

Waterways Experiment

Station

Installation Restoration Research Program

\title{
Technical Approach for In Situ Biological Treatment Research: Bench-Scale Experiments
}

by Mark E. Zappi, Douglas Gunnison, Judith Pennington, M. John Cullinane, Cynthia L. Teeter, James M. Brannon, Tommy E. Myers Environmental Laboratory

Shankha Banerji

University of Missouri-Columbia

Robert Sproull

Oregon State University

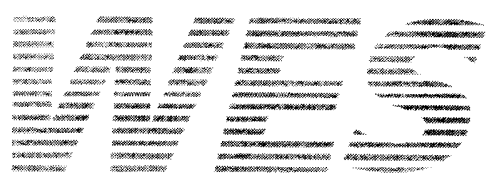

Approved For Public Release; Distribution Is Unlimited 
The contents of this report are not to be used for advertising, publication, or promotional purposes. Citation of trade names does not constitute an official endorsement or approval of the use of such commercial products. 


\section{Technical Approach for In Situ Biological Treatment Research: Bench-Scale Experiments}

by Mark E. Zappi, Douglas Gunnison, Judith Pennington, M. John Cullinane, Cynthia L. Teeter, James M. Brannon, Tommy E. Myers Environmental Laboratory

U.S. Army Corps of Engineers

Waterways Experiment Station

3909 Halls Ferry Road

Vicksburg, MS 39180-6199

Shankha Banerji

University of Missouri-Columbia

Columbia, MO 65211

Robert Sproull

Oregon State University

Corvallis, OR 97331-2702

\section{Final report}

Approved for public release; distribution is unlimited 


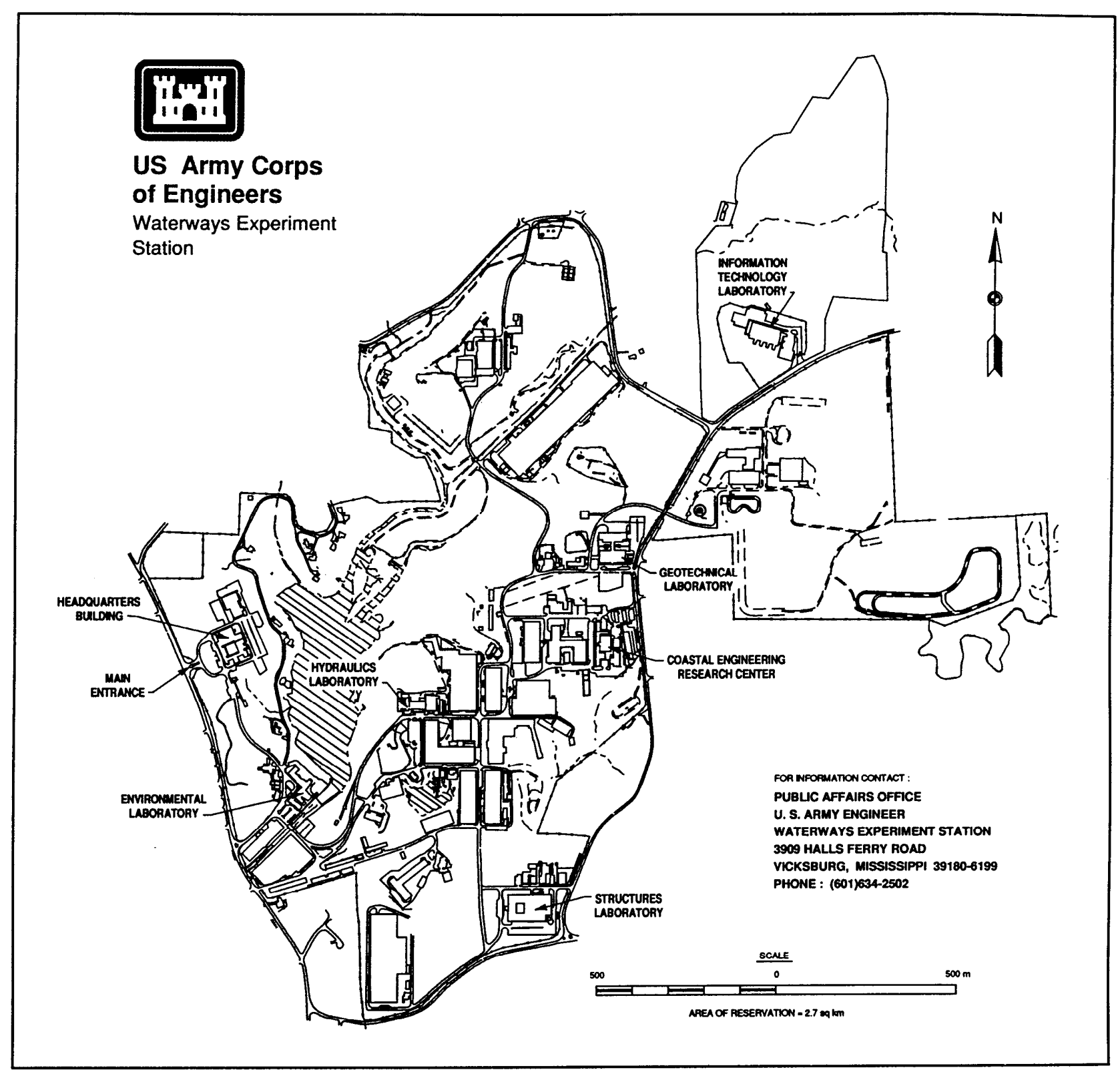

Waterways Experiment Station Cataloging-In-Publication Data

Technical approach for in situ biological treatment research : benchscale experiments / by Mark E. Zappi ... [et al.] ; prepared for U.S. Army Corps of Engineers.

[231] p. : ill. ; $28 \mathrm{~cm}$. - (Technical report ; IRRP-93-3)

Includes report by Robert D. Sproull entitled "Summary of in situ

bioremediation case studies" as Appendix A.

Includes bibliographical references.

1. Water - Purification - Biological treatment. 2. Groundwater -

Purification - Cost effectiveness. 3. Microbial biotechnology. 4. Water

- Purification - Organic compounds removal. I. Zappi, Mark E.

II. Sproull, Robert D. Summary of in situ bioremediation case studies.

III. United States. Army. Corps of Engineers. IV. U.S. Army Engineer

Waterways Experiment Station. V. Installation Restoration Research

Program. VI. Title: Summary of in situ bioremediation studies.

VII. Series: Technical report (U.S. Army Engineer Waterways Experiment Station) ; IRRP-93-3.

TA7 W34 no.IRRP-93-3 
This technical approach was developed by the US Army Engineer Waterways Experiment Station (WES), Vicksburg, MS, as part of the Department of the Army's Environmental Quality Technology Program. The work was conducted in cooperation with the US Army Environmental Center (USAEC). Mr. Wayne Sisk served as the USAEC project manager. Mr. Mark E. Zappi served as the WES project manager. Dr. Clem Myer was the Installation Restoration Research Program (IRRP) Coordinator at the Directorate of Research and Development, Headquarters, US Army Corps of Engineers (HQUSACE). Dr. Bob York, USAEC, and Mr. Jim Baliff, HQUSACE, served as the IRRP Technical Monitors. Dr. John Cullinane, WES, was the IRRP Program Manager.

This technical approach was prepared by Messrs. Zappi and Tommy E. Myers, Dr. M. John Cullinane, and Ms. Cynthia L. Teeter, Environmental Restoration Branch (ERB), Environmental Engineering Division (EED), Environmental Laboratory (EL), WES, and Drs. Douglas Gunnison, Judith Pennington, and James M. Brannon, Environmental Processes and Effects Division, EL, Dr. Shankha Banerji, University of Missouri, and Dr. Robert Sproull, Oregon State University.

The approach was developed under the general supervision of

Mr. Norman R. Francingues, Chief, ERB; Dr. Raymond L. Montgomery, Chief, EED; and Dr. John Harrison, Director, EL.

At the time of publication of this report, Director of WES was Dr. Robert W. Whalin. Commander was COL Bruce K. Howard, EN.

This report should be cited as:

Zappi, Mark, E., Gunnison, Douglas, Pennington, Judith, Cullinane, M. John, Teeter, Cynthia L., Brannon, James M., Myers, Tommy E., Banerji, Shankha, and Sproull, Robert. 1993. "Technical Approach for In Situ Biological Treatment Research: Bench-Scale Experiments, "Technical Report IRRP-93-3, US Army Engineer Waterways Experiment Station, Vicksburg, MS. 
$\underline{\text { Page }}$

PREFACE . . . . . . . . . . . . . . . . . . . . . . . . . . . . . . . . . . . . . . . . . .

CONVERSION FACTORS, NON-SI TO SI (METRIC) UNITS OF MEASUREMENT . . . . $\quad 5$

PART I : INTRODUCTION . . . . . . . . . . . . . . . . . . . . . . . . 6

Background . . . . . . . . . . . . . . . . . . . . . . . . . 6

In Situ Biotreatment Research Program Objective . . . . . . . . . 6

Alternative Physical/Chemical Technologies . . . . . . . . . . . 6

Incineration . . . . • . . . . . . . . . . . . . . . . . . . 7

Air Stripping . . . . . . . . . . . . . . . . . . . . . . . . • 7

Activated Carbon Adsorption . . . . . . . . . . . . . . . . 8

Advanced Oxidation . . . . . . . . . . . . . . . . . . . 8

Low Thermal Stripping . . . . . . . . . . . . . . . . . 8

Biological Treatment . . . . . . . . . . . . . . . . . . . . . . 9

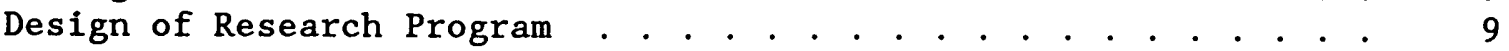

Document Organization . . . . . . . . . . . . . . . . . . . . . . . 9

Program Scope . . . . . . . . . . . . . . . . . . . . . . . . . . 10

Bench-Study Objectives . . . . . . . . . . . . . . . . . . . . . 10

PART II: CONTAMINANTS OF INTEREST . . . . . . . . . . . . . . . . . . . . . . 11

Introduction . . . . . . . . . . . . . . . . . . . . . . . . . . 11

Organic Compound Classes . . . . . . . . . . . . . . . . . . . 11

Chemical Data . . . . . . . . . . . . . . . . . . . . . . . . . 11

Methyl ethyl ketone (MEK) . . . . . . . . . . . . . . . . . 11

Benzene . . . . . . . . . . . . . . . . . . . . . . . . . 12

Ethylbenzene . . . . . . . . . . . . . . . . . . . . . . . 13

Toluene . . . . . . . . . . . . . . . . . . . . . . . . . . . . 14

Xylenes . . . . . . . . . . . . . . . . . . . . . . . . . . . . . . . 14

Trichloroethylene (TCE) . . . . . . . . . . . . . . . . . . 15

Diesel Fuel. . . . . . . . . . . . . . . . . . . . . . 16

2,4,6-Trinitrotoluene (TNT) . . . . . . . . . . . . . . . . 16

$1,3,5$-Hexahydro-1,3,5-Trinitrotriazine (RDX) . . . . . . . 17

PART III: MEDIA OF INTEREST . . . . . . . . . . . . . . . . . . . . . 18

Saturated Soils. . . . . . . . . . . . . . . . . . . . . . . . . 18

Unsaturated Soils . . . . . . . . . . . . . . . . . . . . . . . . 18

Groundwater . . . . . . . . . . . . . . . . . . . . . . . . . 18

Spent Activated Carbon . . . . . . . . . . . . . . . . . . . . . 19

Contaminated Gases . . . . . . . . . . . . . . . . . . . . . . . 19

PART IV: MICROBIAL DEGRADATION PROCESSES . . . . . . . . . . . . . . 20

Objective of Microbial Degradation Section . . . . . . . . . . . 20

Definition of Terms and Major Microbiological Processes . . . . 20

Degradational Processes . . . . . . . . . . . . . . . 20

Types of Microorganisms . . . . . . . . . . . . . . . . . 21

Major Factors Influencing Biodegradation . . . . . . . . . . . . 22

Physical State of the Contaminant . . . . . . . . . . . . 23

Concentration of the Contaminant . . . . . . . . . . . . . 24

Environmental Effects on Microorganisms . . . . . . . . . . 25

Oxygen and Alternate Electron Acceptors . . . . . . . . . . . . . . 30

Nitrate Reduction . . . . . . . . . . . . . . . . . . . . . 30

Manganese Reduction . . . . . . . . . . . . . . . . . . . 31 
Iron Reduction . . . . . . . . . . . . . . . . . . 31

Sulfate Reduction... . . . . . . . . . . . . . 32

Fermentation and Carbon Dioxide Reduction. . . . . . . . . 32

Utilization of Alternate Electron Acceptors . . . . . . . . 32

Microbial Habitats . . . . . . . . . . . . . . . . . . . . . . 33

Role of Competitive Environmental Factors in Degradation of

Contaminants . . . . . . . . . . . . . . . . . . 35

Other Factors Affecting Transformations . . . . . . . . . . . . . 36

Structural Properties of the Contaminant Molecule . . . . . 36

Adequacy of the Microbial Community . . . . . . . . . . . . 36

Mixed Chemicals and Degradation Kinetics . . . . . . . 37

Toxicity . . . . . . . . . . . . . . . . . . . . 37

Adaptation . . . . . . . . . . . . . . . . . . . . . 38

Population Levels of Degrading Microorganisms . . . . . . . 38

Acclimation and Addition of Exotic and Genetically

Engineered Species... . . . . . . . . . . . . . 38

Degradation of Individual Groups of Contaminants . . . . . . . . 40

Microbial Degradation of Volatile Chlorinated Solvents . . . . . 40

Aerobic Degradation... . . . . . . . . . . . . . . . . . 40

Anaerobic Degradation... . . . . . . . . . . . . . . . 42

Discussion and Conclusions . . . . . . . . . . . . . . . . 43

Microbial Degradation of Petroleum Hydrocarbons . . . . . . . . . 44

Degradation of Major Groups of Petroleum Hydrocarbon 45

Components
Microorganisms Degrading Petroleum Hydrocarbons

Degradation of Fuels in Soils . . . . . . . . . . . . . . . 48

Discussion and Conclusions . . . . . . . . . . . . . . . . 50

Degradation of Benzene, Toluene, Ethylbenzene, and Xylene . . . . 51

Aerobic Oxidation . . . . . . . . . . . . . . . . . 52

Anaerobic Degradation... . . . . . . . . . . . . . . . 52

Microorganisms Degrading BTEX Compounds . . . . . . . . . . 53

Discussion and Conclusions . . . . . . . . . . . . . . . . 54

Microbial Degradation of Explosives. . . . . . . . . . . . . . . 55

TNT . . . . . . . . . . . . . . . . . . . 55

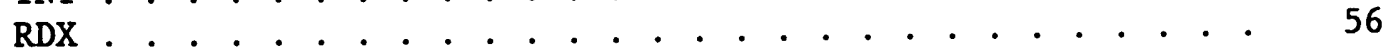

PART V: ADSORPTION/DESORPTION PROCESSES . . . . . . . . . . . . . . 58

Introduction . . . . . . . . . . . . . . . . . . . . . . . . . . 58

Sorption Characteristics of Target Contaminants . . . . . . . . . 59

Adsorption/Desorption Limitations . . . . . . . . . . . . . . . 60

Effect of Sorption on Contaminant Biodegradation . . . . . . . . 62

Sorption of Microorganisms . . . . . . . . . . . . . . . . . 63

Contaminant Desorption Enhancement . . . . . . . . . . . . . . . 64

PART VI: ENGINEERING AND APPLICATION OF BIOTREATMENT SYSTEMS . . . . 66

Introduction . . . . . . . . . . . . . . . 66

Biological Treatment Technologies............. 66

Saturated Soil Biotreatment Technologies . . . . . . . . 66

Unsaturated Soil Biotreatment . . . . . . . . . . . . . . . 84

Activated Carbon Regeneration . . . . . . . . . . . 85

Contaminated Gas Biotreatment . . . . . . . . . . . . . . 91 
PART VII: TECHNICAL APPROACH . . . . . . . . . . . . . . . . . . . . 93

Introduction . . . . . . . . . . . . . . . . . . . . . . . . . . 93

Advisory Committee . . . . . . . . . . . . . . . . . . . . 93

Analytical Chemistry . . . . . . . . . . . . . . . . . . . . 93

Analytical Methods . . . . . . . . . . . . . . . . . . . . . . . 94

Organic Compounds . . . . . . . . . . . . . . . . . . . . . 94

Gross Pollutants . . . . . . . . . . . . . . . . . . . . . 94

Inorganic Compounds . . . . . . . . . . . . . . . . . . . . 94

Miscellaneous Analyses . . . . . . . . . . . . . . . . . . 95

Intermediates . . . . . . . . . . . . . . . . . . . . . 95

Geotechnical Considerations . . . . . . . . . . . . . . . . . . . 95

Research Area Objectives . . . . . . . . . . . . . . . 96

Approach . . . . . . . . . . . . . . . . . . . . . . 96

Pretreatment Technologies . . . . . . . . . . . . . . . . . . . 99

Research Area Objectives . . . . . . . . . . . . . . . . . 99

Approach . . . . . . . . . . . . . . . . . . . . 99

Application of Additives . . . . . . . . . . . . . . . . . . . . 101

Research Area Objectives . . . . . . . . . . . . . . . . . 101

Important Definitions . . . . . . . . . . . . . . . . . . . 102

Approach . . . . . . . . . . . . . . . . . . . . . . 103

Biological Systems Research Area . . . . . . . . . . . . . . 110

Introduction . . . . . . . . . . . . . . . . . . . . . . . 110

Research Area Objectives . . . . . . . . . . . . . . . . . 111

Approach . . . . . . . . . . . . . . . . . . . . . . . . 111

Adsorption/Desorption Processes . . . . . . . . . . . . . . . 131

Research Area Objectives . . . . . . . . . . . . . . . . . 131

Approach . . . . . . . . . . . . . . . . . . . . . . . . . 131

PART VIII: REGULATORY ISSUES . . . . . . . . . . . . . . . . . . . . . 141

Research Objectives . . . . . . . . . . . . . . . . . . . . 141

Approach . . . . . . . . . . . . . . . . . . . . . . . . . . . . 141

REFERENCES . . . . . . . . . . . . . . . . . . . . . . . . . . . . . . 142

TABLES $1-4$

APPENDIX A: IN SITU IMPLEMENTATION CASE STUDIES . . . . . . . . . . . . A1

APPENDIX B: LIST OF ATTENDEES TO THE ADVISORY GROUP MEETING

AUGUST 1990 . . . . . . . . . . . . . . . . . . . . . . . B1

APPENDIX C: SUMMARY OF ADVISORY COMMITTEE MEETING AUGUST 1990 . . . . $\quad$ C1 
CONVERSION FACTORS, NON-SI TO SI (METRIC)

UNITS OF MEASUREMENT

Non-SI units of measurement used in this report can be converted to SI (metric) units as follows:

Multiply

acres

cubic yards

degrees (angle)

feet

tons (nuclear equivalent of TNT)

\begin{tabular}{c} 
By \\
\hline $4,046.873$ \\
0.7645549 \\
0.01745329 \\
0.3048 \\
4.184
\end{tabular}

To Obtain

square metres

cubic metres

radians

metres

gigajoules 


\section{TECHNICAL APPROACH FOR IN SITU BIOLOGICAL TREATMENT \\ RESEARCH: BENCH-SCALE EXPERIMENTS}

PART I: INTRODUCTION

\section{Background}

1. Many US Army installations have areas of contamination requiring environmental remediation. These sites require remedial solutions that are not only technically sound but economically feasible.

2. Typically, a site can be remediated using a variety of decontamination or treatment technologies. A list of technologies capable of meeting the site cleanup goals is normally prepared as part of the remedial investigation/ feasibility study process. From this list, the least costly and environmentally acceptable alternative is usually selected unless other special considerations are incorporated into the decision framework. With the continued development of new and innovative technologies, the "list" of available technologies will continue to grow. The goal is to develop an expanded list of technologies offering equal or better treatment at an appreciable cost savings .

\section{In situ Biotreatment Research Program Objective}

3. The objective of this program is to identify and/or develop technically viable, field implementable in situ based treatment technologies based on biological processes for treatment of contaminated soils and groundwaters, emphasizing in situ technologies. In situ is defined herein as any technology that does not require excavation of contaminated soils. The development of these technologies should result in the reduction of the costs associated with remediating both contaminated soils and groundwaters. This program is part of the Department of the Army's Installation Restoration Research Studies, Environmental Quality Technology Program.

\section{Alternative Physical/Chemical Technologies}

4. A listing of several alternative remediation technologies excluding biological processes is presented below. The intent of this section is to 
give background on technologies that are currently available for site remediation as compared with the biological treatment technologies that will be discussed later in this document.

\section{Incineration}

5. Incineration is a technology that results in the complete destruction of organic compounds using high temperatures. Typical operating temperatures are in excess of $1,500^{\circ} \mathrm{F}$. Incinerators are available in a variety of configurations. For most site remediation applications, incinerators are used for treating contaminated soils and free products; however, incineration of liquids is possible but not usually used to remediate waters from contaminated sites. One major advantage of incineration is that it is a destruction technology that results in the complete destruction of organic contaminants. The major drawbacks to this technology include high cost, poor public acceptance, and regulatory constraints.

\section{Air Stripping}

6. Air stripping is a physical treatment process that does not result in the destruction of the contaminants, but simply relies on contaminant phase change. Air stripping removes contaminants via volatilization from the aqueous phase into the gas phase. The fact that compounds that are amenable to treatment using air stripping technology are those that are volatile is apparent. The higher the contaminant's Henry's Law Constant, the easier the compound is to desorb or "strip" from the aqueous phase into the air phase. For air stripping to be cost-effective, the contaminant must be significantly more volatile than water.

7. Air stripping is commonly used to remove volatile organic compounds from contaminated groundwater. Dissolved cations such as iron can pose significant problems with stripper operation by oxidizing in the column resulting in column blockage. Many states are now requiring treatment of off-gases from air stripping units. This can significantly increase the cost of treatment because of disposal problems associated with spent carbon. Also, recent emphasis on destructive technologies has cast a shadow on the acceptance of this technology by the regulatory community. 
8. Activated carbon adsorption is a popular treatment process in which solutes (contaminants) in wastewater are adsorbed onto the surface of activated carbon. The most used form of activated carbon is granular activated carbon (GAC). The GAC is typically loaded into columns that are referred to as GAC adsorbers.

9. The activated carbon process is not a destruction technology, but a phase change technology in which the contaminants are transferred from the aqueous phase into the solid phase. GAC treatment is attractive because the phase transfer results in the concentration of the contaminants from the liquid phase onto the GAC surface. However, since destruction of the contaminants does not occur, the used carbon must be disposed of, usually as a hazardous waste, or regenerated, which can often be cost prohibitive.

Advanced Oxidation

10. Advanced oxidation processes (AOP) are destruction technologies. These processes typically rely on the synergistic effect of both ultraviolet (UV) light or other catalysts and chemical oxidizers for the destruction of organic compounds. Typical chemical oxidizers are hydrogen peroxide and ozone. The UV wavelength most commonly used is $254 \mathrm{~nm}$. Problems commonly associated with AOPs are fouling of the UV quartz tubes because of solubilized cations and formation of chemical intermediates because of incomplete oxidation of the parent compounds. The electrical power requirements of these systems can also be quite high, resulting in high operational costs.

\section{Low Thermal Stripping}

11. Low thermal stripping uses elevated temperatures to desorb organic contaminants from contaminated soils. This process can be destruction processes if the vapors produced in the desorption device are destroyed in a secondary combustion unit. An example of such a device is the $\mathrm{LT}^{3}$ unit recently field-tested by the Army. A drawback of the technology is that it can be expensive to operate depending on the fate of the gases. Also, saturated soils can be challenging to the technology because all moisture has to be driven off before contaminants can be efficiently desorbed. 


\section{Biological Treatment}

12. Biological treatment offers a cost-effective alternative to traditional site remediation technologies. The technology has been implemented at small localized sites, but, in general, has not been applied to large sites. The United States Environmental Protection Agency (USEPA) recognizes biotreatment as "the" technology of the future. Future is the key word in that statement. There is still much to be done to make this technology a viable technology. The largest knowledge gaps identified are in the areas of applications and determination of technology limitations.

\section{Design of Research Program}

13. The research program is divided into three levels of research: bench-, pilot-, and field-scale testing. The first level of study is the bench-scale studies. This document will present the technical approach for the bench studies.

14. Once the bench studies have been completed, pilot-scale evaluations of various biotreatment technologies are expected based on the results of the bench studies. The technical approach to the pilot studies will be drafted upon completion of key phases of the bench studies. After completion of the pilot studies, those technologies that are deemed technically and economically feasible as a result of the pilot studies will be evaluated during field demonstrations. Separate technical approaches will be drafted prior to implementation of each field demonstration.

15. This research program will concentrate on identifying and solving the practical limitations facing biotreatment technologies. The research performed will be applications orientated and will culminate in the verification of the technology at numerous full-scale demonstrations. If successfully developed, biological treatment or biotreatment can result in significant reductions in the cost of remediating US Army installations.

\section{Document Organization}

16. This document is divided into three main categories of discussion. First, introductory information on the target contaminants and contaminated media that will be evaluated is presented. Second, background information on 
biological processes and factors that directly affect biological processes is presented. The information provided is intended to familiarize the reader with previous accomplishments, to identify knowledge gaps that need to be bridged, and to educate the reader on some of the terminology. Third, the technical approach that will be used to accomplish the goals of the benchscale studies is presented. The logic and methods of how and why the bench studies will attempt to bridge the knowledge gaps discussed in the previous discussions are presented.

\section{Program Scope}

17. The scope of this research program is to evaluate biological processes that do not require excavation of contaminated soils. Pump-and-treat technologies will be evaluated in this research program. Other technically feasible and promising biological technologies such as bioslurry treatment, landfarming, and composting have much promise as alternative treatment technologies, but are not within the scope of this program. These technologies are being researched and further developed as separate work units not covered under this program.

\section{Bench-Study Objectives}

18. The objectives of the bench studies are based on knowledge gaps that have been identified through in-house experience, literature reviews, and interviews with researchers in the area of biological treatment and related fields. The objectives are to:

a. Evaluate potentially feasible biotreatment technologies.

b. Develop and evaluate designs for pilot-scale treatment systems.

c. Develop protocols for evaluating different site remediation alternatives that use biological processes.

d. Assess the biodegradation potential of target organic contaminants that will likely be encountered during remediation of contaminated sites at Army installations.

e. Determine appropriate treatment conditions for optimum biological degradation.

f. Determine if any potentially hazardous end point intermediates are formed during biological degradation. 


\section{Introduction}

19. Based on guidance from the US Army Toxic and Hazardous Materials Agency (USATHAMA) and information gained through discussions with various Army installations, several organic compounds will be evaluated during the course of this research program. These organic compounds are thought to be relatively inclusive of the majority of organic contaminants found at Army installations.

\section{Organic Compound Classes}

20. The contaminants evaluated in this research program will be referred to herein as target contaminants. The target contaminants were broken into classes of contaminants. The contaminant classes and the representative compound(s) that will be evaluated are listed below:

a. Ketones - methyl ethyl ketone.

b. Aromatics - benzene, ethylbenzene, toluene, and xylenes.

c. Petroleum hydrocarbons - diesel fuel.

d. Chlorinated solvents - trichloroethylene.

e. Explosives - TNT, TNT isomers, RDX, and HMX.

\section{Chemical Data}

21. To familiarize the reader with each compound, general information concerning each target compound is presented below. This information, unless noted, was taken from Verschueren (1983).

Methyl ethyl ketone (MEK)

\section{Synonyms: 2-butanone}

Manufacturing Source: Organic chemical industry

Uses: Solvent or swelling agent of resins

Intermediate of ketones and amines production

Flush-off paint stripper 
Extraction fluid of wax from lubricating oil fractions of petroleum

Solvent in nitrocellulose coatings and vinyl films

Cements and adhesives

Smokeless powder production

Printing catalysts and carrier

Cleaning fluids

Properties: Molecular weight: 72.1

Melting point: $-86.4{ }^{\circ} \mathrm{C}$

Boiling point: $79.6{ }^{\circ} \mathrm{C}$

Vapor pressure: $77.5 \mathrm{~mm}$ @ $20{ }^{\circ} \mathrm{C}$

Specific gravity: 0.805 @ $20{ }^{\circ} \mathrm{C}$

Solubility: $353,000 \mathrm{mg} / \ell$ e $10{ }^{\circ} \mathrm{C}$

$\log \mathrm{K}_{\mathrm{ow}}: 0.26$

Odor: Quality: Sweet, sharp

Hedonic tone: neutral to unpleasant

Threshold limit: $32-80 \mathrm{mg} / \mathrm{cu} \mathrm{m}$

Activated Carbon Capacity: $94 \mathrm{mg} / \mathrm{g}$ GAC

Benzene

NOTE: This compound is part of the BTEX group. The "B" in that group is benzene.

Synonyms: None

Manufacturing Source: Petroleum refinery industry, solvent recovery plant, coal tar distillation, coal processing, coal coking.

Uses: Used as component in the manufacture of--

Styrene

Phenol

Detergents

Other organic chemicals

Pesticides

Plastics and resins

Synthetic rubber

Aviation fue 1 
Dyes

Explosives

PCBs

Gasoline

Paints and coatings

Properties: Molecular weight: 78.11

Melting point: $5.5^{\circ} \mathrm{C}$

Boiling point: $80.1{ }^{\circ} \mathrm{C}$

Vapor pressure: $76 \mathrm{~mm}$ @ $20^{\circ} \mathrm{C}$

Specific gravity: 0.879 @ $20{ }^{\circ} \mathrm{C}$

Solubility: $1,780 \mathrm{mg} / \mathrm{l}$ @ $10^{\circ} \mathrm{C}$

$\log \mathrm{K}_{\text {ow }}: 2.13$

Odor: Threshold limit: $0.516 \mathrm{mg} / \mathrm{cu} \mathrm{m}$

Activated Carbon Capacity: $80 \mathrm{mg} / \mathrm{g}$ GAC

\section{Ethylbenzene}

NOTE: This compound is part of the BTEX group. The "E" in that group is ethylbenzene.

Synonyms: Phenylethylene

Manufacturing Source: Organic chemical industry, petroleum refining

Uses: Manufacture of styrene and acetophenone

Solvent

Asphalt constituent

Naphtha constituent

Properties: Molecular weight: 106.17

Melting point: $-94.97{ }^{\circ} \mathrm{C}$

Boiling point: $136.2{ }^{\circ} \mathrm{C}$

Vapor pressure: $7 \mathrm{~mm}$ @ $20{ }^{\circ} \mathrm{C}$

Specific gravity: 0.867 @ $20{ }^{\circ} \mathrm{C}$

Solubility: $152 \mathrm{mg} / \mathrm{l}$ @ $20^{\circ} \mathrm{C}$

Log $\mathrm{K}_{\text {ow }}: 3.15$

Odor: Threshold limit: 2 to $2.6 \mathrm{mg} / \mathrm{cu} \mathrm{m}$

Activated Carbon Capacity: $18 \mathrm{mg} / \mathrm{g}$ GAC 
NOTE: This compound is part of the BTEX group. The "T" in that group is toluene.

Synonyms: Methylbenzene, phenylmethane

Manufacturing Source: Petroleum refining, coal tar distillation

Uses: Used in the manufacture of - -

Benzene derivatives

Saccharin

Medicines

Dyes

TNT

Used in- -

Solvent recovery

Gasoline component

Solvent for paints and coatings

Asphalt and naphtha constituents

Adhesive solvent

Properties: Molecular weight: 92.1

Melting point: $-95.1{ }^{\circ} \mathrm{C}$

Boiling point: $110.8{ }^{\circ} \mathrm{C}$

Vapor pressure: $22 \mathrm{~mm}$ @ $20{ }^{\circ} \mathrm{C}$

Specific gravity: 0.867 @ $20^{\circ} \mathrm{C}$

Solubility: $515 \mathrm{mg} / \ell$ @ $10{ }^{\circ} \mathrm{C}$

$\log K_{\text {ow }}: 2.69$

Odor: Quality: Sour, sweet

Hedonic tone: Neutral to unpleasant

Threshold limit: $0.5-1.0 \mathrm{mg} / \mathrm{cu} \mathrm{m}$

Activated Carbon Capacity: $50 \mathrm{mg} / \mathrm{g}$ GAC

\section{Xylenes}

NOTE: These compounds are part of the BTEX group. The " $X$ " in that group is xylenes.

Synonyms: 0-Xylene: 1,2-dimethylbenzene

m-Xylene: 1,3-dimethylbenzene

p-Xylene: 1,4-dimethylbenzene 
Manufacturing Source: Petroleum distillation, coal tar distillation, coal gas distillation, organic chemical industry

Uses: Manufacture of phthalic and acid anhydride

Terephthalic acid manufacture for polyester

Solvent recovery plants

Gasolines

Protective coatings

Solvents for alkyl resins

Lacquers

Dyes

Asphalt and naphtha constituent

Rubber cements

Properties: Molecular weight: 106.17

$$
\begin{gathered}
\text { Melting point: }-25{ }^{\circ} \mathrm{C} \text { (ortho) } \\
-48{ }^{\circ} \mathrm{C} \text { (meta) } \\
13{ }^{\circ} \mathrm{C} \text { (para) } \\
\text { Boiling point: } 144.4{ }^{\circ} \mathrm{C} \\
\text { Vapor pressure: } 5 \mathrm{~mm} \text { @ } 20{ }^{\circ} \mathrm{C} \text { (o and m) } \\
\quad 6.5 \mathrm{~mm} \mathrm{@} 20{ }^{\circ} \mathrm{C} \text { (p) } \\
\text { Specific gravity: } 0.88 \text { @ } 20{ }^{\circ} \mathrm{C} \\
\text { Solubility: } 175 \mathrm{mg} / \ell \text { @ } 20{ }^{\circ} \mathrm{C} \text { (o and m) } \\
198 \mathrm{mg} / \ell \text { @ } 25{ }^{\circ} \mathrm{C} \text { (p) } \\
\text { Log } \mathrm{K}_{\text {ow }: \quad 2.77 \text { (o and m) }} 3.15 \text { (p) }
\end{gathered}
$$

Odor: Quality: Sweet

Threshold limit: $0.3-1.0 \mathrm{mg} / \mathrm{cu} \mathrm{m}$

Activated Carbon Capacity: $54 \mathrm{mg} / \mathrm{g}$ @ $0.1 \mathrm{mg} / \ell$ (Dobbs and Cohen 1980)

$$
\text { Trichloroethylene (TCE) }
$$

Synonyms: Trichloroethene, ethylenetrichloride

Manufacturing Source: Organic chemical industry

Uses: Dry cleaning

Degreasing activities

Solvents for fats, greases, and waxes

Solvents for dyeing

Refrigerant and heat exchange liquid 
Fumigant

Anesthetic

Properties: Molecular weight: 131.5

Melting point: $-87^{\circ} \mathrm{C}$

Boiling point: $86.7^{\circ} \mathrm{C}$

Vapor pressure: $60 \mathrm{~mm}$ (a $20{ }^{\circ} \mathrm{C}$

Specific gravity: 1.46 @ $20^{\circ} \mathrm{C}$

Solubility: $1.100 \mathrm{mg} / \ell$ @ $10^{\circ} \mathrm{C}$

Odor: Quality: Solventy, ethereal

Threshold limit: $1.0 \mathrm{mg} / \mathrm{cu} \mathrm{m}$

Activated Carbon Capacity: $6.7 \mathrm{mg} / \mathrm{g}$ @ 0.1 feed (Dobbs and Cohen 1980)

Diesel Fuel

Note: This target contaminant is literally made up of many organic compounds. Therefore, there is little information on this hydrocarbon in terms of specific physical/chemical data because the quality of this hydrocarbon can vary with the petroleum source.

$$
\text { 2,4,6-Trinitrotoluene (TNT) }
$$

Synonyms: Sym-trinitrotoluene, 1-methyl-2,4,6-trinitrobenzene, trotyl, tolit, trilit

Manufacturing Source: Explosives manufacture

Uses: Explosives

Intermediates in dyestuffs and photographic chemicals

Properties: Molecular weight: 227.13

Melting point: $88.7{ }^{\circ} \mathrm{C}$ (Urbanski 1964)

Boiling point: $240{ }^{\circ} \mathrm{C}$ explodes (Verschueren 1983)

Vapor pressure: $1.28 \mathrm{E}-6$ torrs @ $20^{\circ} \mathrm{C}$ (Leggett, Jenkins, and Murrman 1977)

Specific gravity: 1.654 @ $20{ }^{\circ} \mathrm{C}$ (Merck and Company 1976)

Aqueous solubility: $200 \mathrm{mg} / \ell$ @ $15{ }^{\circ} \mathrm{C}$ (Verschueren 1983)

Activated Carbon Capacity: Not found 
Synonyms: Cyclonite, hexagen, T4, sym-trimethylenetrinitramine, cyclotrimethylenetrinitramine (Merck and Company 1976)

Manufacturing Source: Explosives manufacture

Uses: Explosives

Properties: Molecular weight: 222.6 (Merck and Company 1976)

Melting point: $205-206^{\circ} \mathrm{C}$ (Merck and Company 1976)

Specific gravity: 1.82 @ $20{ }^{\circ} \mathrm{C}$ (Merck and Company 1976)

Solubility: $42.3 \mathrm{mg} / \ell$ @ $20^{\circ} \mathrm{C}$ (Sikka et al. 1980)

Activated Carbon Capacity: Not found 
22. This research program will evaluate the utility of biological processes for decontamination of five media types: saturated soils, unsaturated soils, groundwater, spend activated carbon, and gases and vapors. These media were selected because they are of significant interest to the Army and their site remediation efforts. The five contaminated media selected for evaluation in this program are listed and discussed below.

\section{$\underline{\text { Saturated Soils }}$}

23. This medium includes the soil and groundwater components of an aquifer. Treatment of this medium is very difficult because of limited accessibility. For the biological treatment study phases of this program, the sources of this medium will be contaminated, saturated soils collected from Army sites. Each target contaminant will have a representative soil selected after review of available soils have been completed and tracked through the pilot-scale studies and culminate with field-scale demonstration at the sites where the soils came from. Collection of these samples is addressed in Part VII of this document.

\section{Unsaturated Soils}

24. This medium consists of three, possibly four (free product), phases and is typically referred to as the vadose zone. The phases associated with the vadose zone are water, soil, gases, and possibly free product(s). The sources and means of collection are identical to those discussed in the saturated soils section.

\section{Groundwater}

25. This medium represents the water phase of saturated soil systems. This medium will be evaluated separately from the saturated soil system because some remediation scenarios such as pump-and-treat only treat contaminated groundwater. However, pump-and-treat activities result in the remediation of both soils and groundwater. The source of the groundwater to be used as test influents will be collected from existing observation wells or 
piezometers in a representative aquifer. If possible, sites selected as sources of the groundwater for bench-scale studies will be used as test sites for the pilot- and full-scale demonstration.

\section{Spent Activated Carbon}

26. Activated carbon is used at many Army installations as a means of decontaminating aqueous and gaseous streams. Once all of the adsorption sites on the activated carbon are occupied, the activated carbon is no longer useful for decontamination of the waste streams. Completely loaded carbon is commonly referred to as being "spent." The spent carbon is either disposed of as a hazardous waste, which is expensive, or regenerated using physical treatment technologies, such as incineration or wet air oxidation, which are also expensive. Biological treatment is an attractive alternative method for regeneration of spent carbon.

27. The source of the spent carbon for this program will be spent carbon that is generated using synthetic influents in the laboratory. The procedures used in generating the spent carbon contaminated with appropriate contaminants is detailed in Part VII.

\section{Contaminated Gases}

28. During remediation of contaminated groundwater and/or soils using both abiotic and biotic treatment systems, contaminated gas streams are generated that usually require treatment. Examples of these streams include offgasing from: (a) air stripping units, (b) low temperature thermal treatment systems $\left(\mathrm{LT}^{3}\right)$, and (c) in situ vacuum extraction systems. Contaminated gas streams from process equipment as the $\mathrm{LT}^{3}$ and air strippers are currently decontaminated using adsorption or thermal destruction systems. These systems are expensive and, in the case of activated carbon, nondestructive.

29. The sources of contaminated gases used in this research program will be artificially generated from laboratory systems. More detail on the proposed methods for generating the contaminated gases used in this research program is discussed in Part VII of this document. 


\section{Objective of Microbial Degradation Section}

30. The objective of this section is to review existing information on the capabilities and limitations of microbial degradation of contaminants. This information will then be evaluated within the context of in situ bioremediation of contaminated Army sites. The information presented here was obtained from the literature and through conversations with leading authorities in the field.

\section{Definition of Terms and Major Microbiological Processes}

\section{Degradational Processes}

31. The pathways used by microorganisms to break down contaminants are as complex and varied as the contaminants themselves. The purpose of this section is to provide an overview of the biodegradation pathways; these are described in detail in other sources (Gibson and Subramanian 1984). Details of the pathways are changing daily as increased knowledge is gained concerning the fate of various contaminants. Major steps in the degradation process for the groups of contaminants considered herein will be examined in the sections on the individual groups of contaminants. A number of terms and concepts are defined here because of their importance to the degradation process.

32. According to Larson (1984), biodegradation processes may be separated into two categories, primary degradation or biotransformation and ultimate biodegradation or mineralization.

a. Biotransformation - Primary biodegradation occurs when a discrete alteration is brought about in the structure of a chemical resulting in a loss of the basic physical-chemical properties. Larson (1984) gives as examples the hydrolytic cleavage of an ester group from malathion to form $\beta$-monoacid and the cleavage of an acetate group from nitrilotriacetic acid to form the diacid iminodiacetic acid.

b. Mineralization - Mineralization is the regeneration of inorganic forms of nutrients, primarily carbon, nitrogen, phosphorus, and sulfur, through decomposition of organic compounds. During the mineralization process, the organic compounds serve as sources of matter and energy for the microorganism carrying out the degradation. During this process, one of the terminal carbon atoms comprising the molecule may undergo the following 
sequence of changes: aldehydes to alcohols, then finally to a carboxyl group. At this point, the carbon can be released from the molecule as carbon dioxide (NOTE: This sequence is highly simplified. Many more processes are involved in the various steps presented). Under anaerobic conditions, portions of the compound containing carbon may also be released as methane.

c. Oxidation-Reduction Reactions - Microorganisms obtain their energy either from sunlight (not of concern here) or chemical energy supplied by either organic or inorganic compounds. Using a chemical substance as an energy source involves oxidation-reduction reactions wherein the energy source is oxidized and loses electrons, while another substance, the electron acceptor, is reduced and takes up electrons in the process. Either organic or inorganic compounds can serve as sources of electrons (electron donors).

d. Dehalogenation - Dehalogenation is the removal of halogen groups from a contaminant; halogen groups (or moieties) may prevent access to the nonhalogenated portion of the molecule by microbial enzymes required to degrade the compound. For this reason, dehalogenation is often a rate-limiting step in degradation. Dechlorination of aromatic compounds can occur by reductive dehalogenation (Suflita et al. 1982), hydroxylsubstitution, including hydrolytic and oxygenolytic dehalogenations (Thiele, Muller, and Lingens 1987), or by oxidation (Focht and Brunner 1985). Specific pathways of degradation are determined by the environment and the biological capabilities of the resident microorganisms.

e. Cometabolism and Cooxidation - The phenomenon of cometabolism was examined by Horvath (1972) and refers to a microbially mediated alteration in the chemical structure of a contaminant molecule so that the molecule is changed, but not to an extent sufficient to provide the responsible microbe with energy or nutrients. However, other microorganisms may then be able to degrade the modified compound, obtaining nutrients and energy in the process. As discussed by Keck et al. (1989), during the process of cooxidation, hydrocarbons not used for growth may be oxidized when present as cosubstrates in an actively growing culture where one or more different hydrocarbons are supplied for growth. Horvath (1972) distinguished cometabolism from cooxidation by using cometabolism to refer to the oxidation of any nongrowth substrate, regardless of whether or not a growth substrate is present. In this sense, cooxidation is a subset of cometabolism.

\section{Types of Microorganisms}

\section{Aerobic microorganisms}

33. Aerobic microorganisms require oxygen as a terminal electron acceptor in respiration and for biosynthesis of fatty acids (Brock 1970). Organisms utilizing organic compounds as electron donors are referred to as 
heterotrophs, while those obtaining all of their energy from sources other than organic compounds are termed autotrophs. Many bacteria, and most fungi, algae, and protozoa are obligate aerobes--i.e., they require oxygen for growth. Lack of sufficient levels of oxygen in a medium can often be responsible for poor growth of aerobic microorganisms; this can be brought about by a number of different factors (Reddy, Rao, and Patrick 1980).

\section{Anaerobic microorganisms}

34. Anaerobic microorganisms utilize biochemical reactions wherein oxidized compounds serve as electron acceptors and are reduced. This process is fueled by the oxidation of organic or inorganic compounds. In natural systems, reduction of inorganic compounds follows a stepwise sequence predicted by thermodynamics. Once almost all dissolved oxygen has been utilized, facultatively anaerobic bacteria, capable of growth in both aerobic and anaerobic environments, take over from aerobic microorganisms, and other electron acceptors are used in place of oxygen. Initially, nitrate is reduced. Upon consumption of all nitrate supplies, manganese IV is reduced, followed by iron III, sulfate, and then carbon dioxide. Most obligate anaerobes use organic materials to produce carbon dioxide and methane, and some are extremely intolerant of oxygen. Major features of these reduction steps are discussed in detail by several authors (Yoshida 1975; Ponnamperuma 1972) and are briefly summarized in the section on oxygen and alternate electron acceptors.

\section{Major Factors Influencing Biodegradation}

35. This section highlights the physical, chemical, biological, and other environmental factors necessary to bring about biodegradation of the contaminants of concern for this project. The questions being addressed are as follows: (a) Can microorganisms degrade the contaminant either in situ or in a pump-and-treat situation? (b) What substances can be supplied to the populations in aquifer soils, groundwater, or the vadose zone to encourage degradation? (c) What conditions in a pump-and-treat system can be altered to promote development of degrading populations of microorganisms? The next sections consider environmental factors and microbial requirements that serve to enhance or limit biodegradation. 
36. The physical state of the target contaminant has a strong influence on the availability. of that substance to microbiological degradation. Pure compounds present in a solid, liquid, or gaseous state tend to persist because (a) microorganisms are unable to directly interact with the compound in one of these states, (b) microorganisms require water and nutrients that are not present within the matrix of the pure solid, liquid, or gas, and (c) the target compound in concentrated form may be toxic to an organism otherwise capable of using the substance in dilute solution (Inoue and Horikoshi 1989; personal communication with Dr. Martin Alexander, Corne11 University 1990). As a result, microbial utilization of compounds that are pure solids, liquids, or gases either does not occur or else is restricted to interfaces between compounds in these states and water containing suitable nutrients.

37. Petroleum hydrocarbons, for example, are one group of compounds susceptible to degradation by microorganisms and have components present as solids, liquids, or gases. Microorganisms attacking paraffins in the solid state or in oil or gasoline (liquid) appear to live in an adjacent water layer, obtaining their substrates either from compounds that slowly dissolve into the aqueous phase from the solid or liquid or from compounds released into the aqueous phase by a microbially produced surfactant (personal communication with Dr. Martin Alexander, Cornell University, 1990). Also, the possibility exists that some microbes can utilize organic compounds directly from nonaqueous phase liquids.

38. The exact mechanism for utilization of compounds in a gaseous phase is not known, but it is believed that the gaseous compound must either first dissolve into water or else the compound is used by a microorganism having immediate access to water. The microbiologist often uses this to his advantage to isolate microorganisms capable of growth on volatile hydrocarbons. A common practice in petroleum microbiology is to streak a source containing microorganisms onto a petri plate containing an inorganic salts solution in an agar gel; the plate is then placed into a closed container where the atmosphere becomes saturated with fumes of the test hydrocarbon(s). Organisms capable of using the hydrocarbon are the only ones with access to a carbon and energy source and, therefore, are the only microorganisms able to grow under these conditions. 
39. When the contaminant is present in a dissolved state, the concentration will have a bearing on its susceptibility to microbial attack. If the compound is present at a level acceptable to a microorganism able to bring about its degradation, the organism will use the target chemical at moderate to high concentrations of the chemical (gratuitous degradation). There are also cases where the compound may be used at lower levels, as is the case with acetone; but high levels of the compound may prevent degradation if the compound is toxic to the organism (see above and also Phelps et al. (1988)). There are cases where one organism may tolerate concentrations lethal to most other species (Inoue and Horikoshi 1989).

40. If an organism capable of degrading a contaminant does not encounter concentrations of the substrate high enough to support growth, degradation may not occur because of the need for the microbes to grow in order to cause degradation (Boethling and Alexander 1979; Zaidi, Murakami, and Alexander 1988). Alternatively, if organisms destroy the compound by cometabolic mechanisms, but lack sufficient cosubstrates to support growth, then the enzymes needed to break down the contaminant will not be produced.

41. Dr. Alexander (personal communication 1990) has indicated that no microorganisms are known to be capable of growth on compounds below the partsper-billion range (ppb). He further suggests that while lack of growth at less than $5 \mathrm{ppb}$ does tend to limit degradation, demonstrated activity occurs at concentrations well below the threshold for growth--i.e., down to one part per trillion. One explanation for biodegradation of organic compounds below the threshold level is that the organisms are using higher concentrations of other compounds for maintenance energy and growth while simultaneously consuming the contaminants. Second, substrates and uncharacterized dissolved organic carbon may play an important role in controlling the rate and extent of biodegradation of organic contaminants at low concentrations (Schmidt and Alexander 1985). LaPat-Polasko, McCarty, and Zehnder (1984) reported secondary substrate utilization of methylene chloride by a Pseudomonas sp. Using both batch and continuously fed reactors, they demonstrated that this strain was capable of simultaneously consuming the substrates at different concentrations; the primary substrate, acetate, at the higher ppm level (milligrams per liter) and the secondary substrate (methylene chloride) at the lower ppb level 
(micrograms per liter). The methylene chloride was found to be inhibitory at a level of ca. $10 \mathrm{mg} / \mathrm{l}$.

Environmental Effects on Microorganisms

\section{Sorptive Effects}

42. The physical and chemical factors responsible for the resistance or susceptibility of organic compounds to biodegradation were considered in detail by Alexander (1965). Compounds are believed to be more susceptible to biodegradation in the dissolved state, and sorption is considered to limit the rate of biodegradation for many compounds (Rao 1990; Robinson, Farmer, and Novak 1990). Moreover, biodegradation kinetics are known to be affected when intraparticle mass transfer of contaminants controls the rates of desorption and biomineralization of organic contaminants (Rijnaarts et al. 1990). A detailed consideration of the effects of sorption on contaminant degradation is given in Part $\mathrm{V}$.

43. Dr. Perry McCarty of Stanford University (personal communication 1992) gave a specific example of the effects of sorption on the kinetics of degradation of sorbed contaminants. The example demonstrated that if a target contaminant must be desorbed before degradation can occur, physical-chemical factors rather than microbiological factors are limiting degradation. In one study, radiolabeled chlorobenzene in water was moved through a column of activated carbon. If oxygen was also added to the water moving through the column, a microbial population developed, the radiolabeled chlorobenzene was no longer sorbed, and radiolabeled $\mathrm{CO}_{2}$ was formed. If the oxygen flow was stopped, degradation stopped, radiolabeled $\mathrm{CO}_{2}$ was no longer formed, and radiolabeled chlorobenzene was again sorbed to the column. If the supply of radiolabeled chlorobenzene was stopped and oxygen flow restarted, a slow return to a low rate of $\mathrm{CO}_{2}$ production occurred, suggesting microbial degradation of the desorbing radiolabeled chlorobenzene (personal communication with Dr. Perry McCarty, Stanford University 1990).

44. The effects of sorption on the biodegradation of compounds mean that simple studies will be ineffective in predicting the degradability of a contaminant in the environment. According to Dr. Alexander (personal communication 1990), degradation of a compound in a defined medium containing the target compound together with a pure culture of a degrading microorganism is often not difficult to achieve in the laboratory. However, the same compound 
may persist for years in the field because of a combination of factors, of which sorption is one of the most important. An example of this is dibromochloropropane (DBCP), a compound that can be made to degrade in the laboratory but persists for years in groundwater. Another group of examples with large apparent differences in behavior between laboratory and field is the polycyclic aromatic hydrocarbons (Kayal and Connell 1990). Unfortunately, most microbiologists work with highly soluble compounds, but many of the contaminants of interest to the Army are poorly soluble. Generally, the more soluble the target contaminant, the more manageable the degradation process because, as the degrading microbe consumes the target contaminant, more contaminant is pulled into solution by the partitioning process. Microbial limitations in soil

45. Dr. Alexander (personal communication 1990) also indicated that there are many other facets to the sorption-desorption problem. In addition to the actual sorption of a contaminant to soil, there is the problem of diffusion within the soil matrix. While soil can be a highly porous medium, the microorganism must be able to get to the substrate. Moreover, tortuosity increases the difficulty for the organism and target compound to get together by increasing the complexity of the path as well as the physical distance between microbe and substrate. Also, many microorganisms are physically unable to get through micropores in the soil and therefore cannot get to the substrate.

46. According to Dr. Alexander (personal communication 1990), another mechanism whereby a microbial cell that is too large to move through soil pores may obtain its target compound is through the release of surfactants. These compounds may solubilize the compound, making it available for attack. Also, certain microorganisms may have the ability to use the compound within a solvent phase that permits direct physical contact between the organism and the compound (see Part V).

\section{other physical factors}

47. Redox potential and $\mathrm{pH}$ have strong influences on the activity of degrading microorgansisms. Redox potential is an indication of the intensity of oxidizing or reducing conditions present within the flooded soil/sediment environment. As indicated in Ponnamperuma's (1972) review, redox potentials of $+400 \mathrm{mV}$ and above are indicative of the presence of dissolved oxygen, a condition required for the activity of aerobic microorganisms. Redox values below this indicate various stages of low oxygen to anaerobic conditions. As 
indicated above, microbial activities utilizing various elements or compounds as electron acceptors tend to "poise" the redox potential at certain values that are maintained as long as the reduction activity continues. Redox values within the range of +220 to $+330 \mathrm{mV}$ permit denitrification. Manganese reduction occurs around $+200 \mathrm{mV}$, iron reduction at $+120 \mathrm{mV}$, and sulfate reduction between +50 and $-150 \mathrm{mV}$. Below $-150 \mathrm{mV}$, methanogenesis is a prevalent microbiological process. While these redox values can sometimes be used to indicate the reduction of specific electron acceptors, this is not always possible. A variety of organic redox couples are often present in flooded soils and sediments that tend to provide redox readings not in accordance with those expected based on the reduction of inorganic compounds. For this reason, it is not always possible to determine whether active reduction of inorganic electron acceptors is occurring in the soil matrix based on the redox potential.

48. Redox becomes important to the degradation of contaminants when the soil environment has redox values significantly different from the value needed for maximum degradation. Thus, if a contaminant is degraded only under aerobic conditions and anaerobic conditions prevail within the soil matrix, some means must be found to introduce oxygen. Alternatively, if methanogenic conditions are required for decomposition, but active denitrification is occurring, a different solution must be found.

49. While the soil $\mathrm{pH}$ usually does not greatly affect biodegradation, in some cases, $\mathrm{pH}$ may affect microbial activity. Anaerobic degradation proceeding in a poorly buffered soil may result in accumulation of organic acids with a consequent drop in $\mathrm{pH}$ (Ponnamperuma 1972). The increased acidity may gradually eliminate the activity of the microbe carrying out a desired degradation.

50. Rates of water flow may also be important in degradation. Novak et al. (1989) were able to characterize underground sites as either "fast" or "slow," depending on the rate of water movement through them. Fast sites had a high water flow, developed a diverse and responsive microbial population, and featured degradation of a wide variety of organic compounds. Addition of nitrate to such sites stimulated degradation, possibly by a large population of resident denitrifiers. Slow sites had a limited flow of water and were apparently dominated by sulfate-reducing and methanogenic organisms. Addition of molybdate (an inhibitor of sulfate reduction) to these sites frequently stimulated growth of methanogens, suggesting that sulfate-reducing organisms 
outcompeted other organisms for hydrogen. Addition of nitrate to these sites had little or no effect on degradation; alternatively, degradation was sometimes inhibited by nitrite accumulation.

51. Temperature can have unforeseen effects on degradation. Temperature is generally not of concern within the soil matrix because soil temperatures, while generally low, are often quite stable. However, it is important to understand the consequences of changes in temperature, particularly when aboveground treatment processes are to be utilized. Increases in temperature are usually expected to double the microbial degradation rate for every $10{ }^{\circ} \mathrm{C}$ increase in temperature $Q_{10}$ effect. However, there are exceptions, particularly at temperatures below $20^{\circ} \mathrm{C}$. Thus, Best et al. (1990) found that biodegradation rates for a plant material underwent substantially larger increases over the temperature range from 5 to $10^{\circ} \mathrm{C}$ (20 percent) than in the range of 10 to $18{ }^{\circ} \mathrm{C}$ ( 2 percent). Others have found temperature has variable effects on degradation according to the parameters being examined. For example, warmer temperatures might be expected to increase the volatilization of lower molecular weight compounds, resulting in a decrease in biodegradation because of decreased substrate concentration. However, Walker and Colwell (1974) reported that the decreased toxicity present at lower temperatures may actually enhance biodegradation of petroleum. Atlas (1981), based on his review, suggests that lower temperatures may retard volatilization of low molecular weight compounds that inhibit degradation.

\section{Limiting nutrient concentrations}

52. Several important conditions are required for biodegradation of contaminant compounds. According to Alexander (1981, 1985) these include: (a) a target compound having structure, solubility characteristics, and concentrations amenable to degradation; (b) a suitable number of microorganisms having the ability to break down the target compound; (c) conditions supporting the growth of the degrading microorganisms, including the presence of suitable growth substrates and other nutrients, suitable $\mathrm{pH}$, temperature, lack of hostile microorganisms, etc; and (d) accessibility of the degrading microorganism(s) to the target substrate.

53. Once these conditions have been met, the next requirement is environmental conditions conducive to the growth of the degrading microorganism. of particular importance here are the nutrients required by the microorganisms carrying out the desired biodegradation. In contrast to microorganisms in surface soils and sediments, microorganisms in subsurface environments are 
apparently adapted for survival and growth under nutrient-limited conditions (Ghiorse and Balkwill 1983). Since subsurface environments are often oligotrophic (low nutrient levels), native populations of subsurface microorganisms likely live under low-nutrient stressed conditions (Ghiorse and Balkwill 1983). In addition, subsurface microorganisms are often adapted to comparatively low temperatures and have relatively low metabolic rates (Grbic-Galic 1990). Thus, to facilitate biodegradation by native populations of microorganisms in situ, it is important to supply the nutrients necessary to stimulate growth of microorganisms capable of carrying out the desired degradational activity. Most often, the limiting nutrients will be nitrogen and/or phosphorus.

54. Addition of the requisite nutrients to the contaminated soil or groundwater may improve microbial destruction of the contaminants. Organic and inorganic nutrients in natural waters are known to affect the rate of mineralization of compounds in trace concentrations (Rubin and Alexander 1983), and this may also be true for low levels of organic contaminants in the soil. Rubin and Alexander found that inorganic nutrients, arginine, or yeast extract often enhanced, but glucose reduced the rate of phenol mineralization. Mineralization was slower than expected at phenol levels of $1.5 \mathrm{pg} / \mathrm{m} \ell$ or less, but occurred at predicted rates in the presence of arginine. Based on these results, increasing nutrient levels in natural environments may enhance degradation directly or by supporting a larger, more diverse microbial community that has a greater chance of containing microorganisms having the requisite degradational capabilities.

55. Addition of organic nutrients may cause problems, however, if these chemicals select for nondegrading species of microorganisms. Moreover, placing nutrients into the ground will not guarantee improved biodegradation rates, even if studies show that this should occur. Diffusion may limit degradation, while overall nutrient levels may not be limiting. Microorganisms involved in active degradation may be growing at some location where the rates of diffusion are inadequate to supply sufficient nitrogen and phosphorus to provide optimal concentrations of $C$ and $P$ for microbial growth and metabo1ism. In addition, the soil system must be considered a nutrient-limiting environment (Brunner and Focht 1984); while the concentration of nutrients such as nitrogen and phosphorus may appear to be adequate, their availability for microorganisms can still be low because of adsorption at exchange sites in the soil (Marshall 1971). In this case, soil microorganisms having a low $k_{m}$ 
for uptake of the target contaminant would do just as well as those having higher $k_{m}$ values even after addition of nitrogen and phosphorus to the soil because even the added nutrients would still be comparatively unavailable.

\section{Oxygen and Alternate Electron Acceptors}

56. All microorganisms require oxygen or some other electron acceptor to carry out their biodegradational activities. The fermentative bacteria and either facultative or obligate anaerobes carry out metabolism of various substrates producing organic acids, $\mathrm{CO}_{2}, \mathrm{NH}_{4}$, and sulfide (fermentative organisms) or $\mathrm{CO}_{2}$ and $\mathrm{CH}_{4}$ (anaerobes), respectively (Thimann 1963). In general, utilization of electron acceptors present in soil occurs in a stepwise sequence as outlined above.

\section{Nitrate Reduction}

57. Microbial reduction of nitrate may be either assimilatory or dissimilatory in nature. The product of assimilatory nitrate reduction, ammonium, is incorporated into the cell (Payne 1973). In dissimilatory nitrate reduction, the reduced product, ammonium, is released rather than being incorporated into the cell, and the process is coupled with microbial energy production (Hasan and Hall 1975). While many microorganisms may carry out dissimilatory reduction of nitrate to ammonium, only a limited number have been examined for their ability to conduct this process (Sorensen 1978).

58. Respiratory reduction of nitrate to molecular nitrogen is used as an alternative to oxygen respiration by denitrifying bacteria in aquatic systems having dissolved oxygen levels less than $0.2 \mathrm{mg} / \ell$ (Knowles 1979). In the absence of dissolved oxygen, nitrate in water stabilizes or "poises" the oxidation-reduction (redox) potential at approximately $250 \mathrm{mV}$. Reduction of nitrate to nitrogen is especially observed where anaerobic zones and oxidized soil are in close proximity or at sediment surfaces (Koike and Hattori 1978; Sorensen 1978). Ammonium is oxidized to nitrite and nitrate in aerobic areas. Nitrites and nitrates diffuse into areas of lower oxygen levels, where they are reduced to molecular nitrogen (denitrification) (Reddy and Patrick 1975). The initial step in nitrate reduction, reduction of nitrate to nitrite, can be carried out by a variety of bacteria. The ability to carry out complete 
reduction of nitrate to molecular nitrogen is apparently found in both heterotrophic and autotrophic bacteria (Alexander 1977).

Manganese Reduction

59. While manganese dioxide can be used as an electron acceptor for anaerobic respiration at an oxidation level similar to that of nitrate, manganese dioxide is not as effective as nitrate in stabilizing the redox potential (Patrick and Mikkelsen 1971; Ponnamperuma 1972). Inability to stabilize the redox potential may be a result of the very limited solubility of manganese dioxide (Ponnamperuma 1972). Nonetheless, many soil fungi and bacteria are capable of reducing oxidized forms of manganese (Bautista and Alexander 1972). When nitrate and manganese dioxide are both present in anaerobic media, nitrate is used preferentially (Takai and Kamura 1966).

\section{Iron Reduction}

60. The concentration of iron is much greater than nitrate and manganese in soils. Evidence that microbial activity is responsible for iron-III reduction is provided by increased reduction rates corresponding to the addition of organic matter to soil or an iron oxyhydroxide slurry (Gotoh and Patrick 1974). The effect on redox potential of adding iron oxyhydroxides is not as pronounced as the effect of adding manganese dioxide (Nhung and Ponnamperuma 1966). Moreover, addition of manganese dioxide or nitrate to a flooded soil system undergoing iron-III reduction retards the reduction process (Takai and Kamura 1966). The mechanism for this process is not understood; however, addition of nitrate to anaerobic microcosms rapidly oxidizes reduced forms of iron and manganese, suggesting a chemical oxidation process. Iron reduction is a capability of soil microorganisms, occurring in as many as $10^{4}$ to $10^{6}$ bacteria per gram of soil (Alexander 1977). Microbial reduction of iron-III is not only a major process in the mineralization of organic matter in aquatic systems (Lovely and Phillips 1986), but one isolate has been known to couple oxidation of aromatic compounds to iron reduction (Lovely and Lonergan 1990). 
61. Sulfate reduction is very important for the production of sulfide in natural ecosystems, particularly marine and estuarine environments. However, a narrow $\mathrm{pH}$ range $(6.8$ to 7.1$)$ and a low redox potential ( $-150 \mathrm{mV}$ ) are necessary for reduction to occur (Connell and Patrick 1968). Sulfate-reducing and methanogenic microorganisms apparently compete strongly for organic substrates, particularly acetate, and also for hydrogen (Winfrey and Zeikus 1977; Lovely, Dwyer, and Klug 1982; Lovely and Klug 1983; Winfrey and Ward 1983). Sulfate reduction can support degradation of organic contaminants (Suflita, Gibson, and Beeman 1988). Sulfate reduction is believed to be carried out primarily by members of the genus Desulfovibrio, which contains species capable of both autotrophic and heterotrophic growth and by members of the genera Desulfomonas and Desulfotomaculum (Gottschalk 1979). Sulfate reduction is inhibited by addition of nitrate, manganese dioxide, and iron (III) phosphate (Engler and Patrick 1973). Manganese dioxide is incapable of competing with sulfate as an electron acceptor, but oxidizes any hydrogen sulfide produced.

\section{Fermentation and Carbon Dioxide Reduction}

62. Fermentation is a low energy-yielding process (Sokatch 1969) that anaerobically converts organic compounds to organic acids, alcohols, and carbon dioxide (Gottschalk 1979). Major gas products released to soils by fermentative processes include carbon dioxide, nitrogen, hydrogen, and methane. Methanogenesis, the reduction of carbon dioxide to methane, is considered by some authors to be a utilization of an alternate electron acceptor since some methanogens carry out their activity by reducing carbon dioxide. However, other investigators consider methanogenesis to be fermentation (Gottschalk 1979), since methane may also be produced by the decarboxylation (transmethylation) of acetate. Methanogenesis becomes a significant terminal electron acceptor once all sulfate has been depleted.

\section{Utilization of Alternate Electron Acceptors}

63. Recent studies have considered microbial utilization of alternate electron acceptors to support biodegradation. Most of the studies have used nitrate to replace oxygen. Some examples of alternate electron acceptors used 
by microorganisms in the mineralization of contaminants are nitrate, sulfate, and carbon dioxide.

64. Kuhn et a1. (1985) found that nitrate serves as an electron acceptor for dimethylbenzene. Zeyer, Kuhn, and Schwarzenbach (1986) reported rapid microbial mineralization of toluene and 1,3-dimethylbenzene by microorganisms using nitrate as an electron acceptor. Mihelcic and Luthy (1988) reported nitrate-supported degradation of acenaphthene and naphthalene from levels of several $\mathrm{mg} / \ell$ to below detection in less than 9 weeks. Microbial degradation of polycyclic aromatic hydrocarbon (PAH) compounds apparently depends on interrelationships between desorption kinetics and the reversibility of desorption of the sorbed compound from soil, the concentration of PAHdegrading microorganisms, and competing reactions for nitrate utilization with mineralization of labile portions of the natural soil organic matter. Bouwer and McCarty (1983a) observed transformation of carbon tetrachloride and brominated trihalomethanes after 8 weeks in batch denitrification cultures.

65. Utilization of a variety of electron acceptors was reported by Kuhn et al. (1989), who examined the metabolic fate of hydroxybenzoate isomers in soil slurries from a shallow anoxic aquifer. When the slurries were incubated under denitrifying, sulfate-reducing, and methanogenic conditions, the anaerobic fate of the hydroxybenzoate isomers depended on the relative substitution pattern of the isomers and on prevailing ecological conditions within the slurries. While the same metabolites were produced from a given isomer under sulfate-reducing and methanogenic conditions, addition of nitrate to slurries sampled from these sites resulted in transformation of all substrates to phenol. Complete mineralization of hydroxybenzoates coupled with denitrification occurred within 1 month. Toluene and benzene are apparently transformed by mixed methanogenic cultures derived from ferulic acid-degrading sewage sludge enrichments (Grbic-Galic and Vogel 1987). These cultures utilize exogenously supplied $\mathrm{CO}_{2}$ for methanogenesis, but also release $\mathrm{CO}_{2}$ during the anaerobic degradation process. Other investigators have also reported degradation of organic contaminants under methanogenic conditions (see Grbic-Galic et al. (1990)).

\section{Microbial Habitats}

66. As is evident from the preceding information, microorganisms have a high affinity for surfaces. Microorganisms added to groundwater are very 
likely to sorb to the soil, unless the aquifer lacks sorptive surfaces. Degradation may be affected by either sorbed or free-living microorganisms; however, degradation is apt to be poor if the target compound has an affinity for aquifer soils.

67. Aquifer soils that are always saturated with water tend to be anaerobic, unless water moves through them quickly and the organic content of the subsurface environment is low. Native microorganisms capable of degrading target contaminants are most likely to be facultative or obligate anaerobes. If degradation of the target contaminant is best accomplished by aerobic microorganisms, much effort will be required to supply oxygen (and perhaps also microorganisms) to promote degradation in situ.

68. Vadose zone soils, by virtue of their alternate wet and dry status, can place a great deal of stress on microorganisms. At least some moisture is required by all microorganisms to carry out life processes. Bacteria require very moist environments, although many soil bacteria have resting forms (spores) capable of enduring dry periods. Fungi are more tolerant of low moisture levels and, in fact, do poorly in situations where water is abundant and dissolved oxygen levels are low. On the other hand, vadose zone soils are much more likely to have abundant levels of oxygen and will generally experience anaerobic conditions at infrequent intervals. In the vadose zone, aerobic microorganisms can best carry out the destruction of suitable target compounds .

69. Groundwater is a fairly good habitat for microorganisms, although, as indicated above, sorptive surfaces and the filtering effect of porous soils may remove many microorganisms. For this reason, the total microorganisms present in groundwater may be very low compared with numbers in the surrounding soil. Whether these microorganisms are aerobes or anaerobes depends on the nature of the aquifer. Solubility limitations may prevent free-living microorganisms from contacting large amounts of a target contaminant, thereby minimizing degradation. By contrast, investigations by Arvin et al. (1988) suggest that free-living microorganisms are very important to degradation and that there is a requirement for coarse rather than fine soil fractions to promote interactions between substrate and microorganisms. 


\section{Role of Competitive Environmental Factors}

in Degradation of Contaminants

70. Several other environmental factors may act either independently or synergistically to influence biodegradation. Abiotic losses of the target contaminant may compete with microorganisms for substrates, but abiotic losses may also have toxic or inhibitory effects. For example, volatilization can remove low molecular weight toxic components in a petroleum hydrocarbon spill, but this type of volatilization may be retarded at temperatures low enough to prevent degradation (Atlas 1981). Moreover, degradation of the residual phases in a petroleum hydrocarbon spill may be inhibited by volatilization (Atlas 1981). Also, rapid evolution of gases in soil, either through man's activities or microbial processes (denitrification, methanogenesis, $\mathrm{CO}_{2}$ evolution) may strip volatile contaminants from the soil before microorganisms have the opportunity to degrade them. Another abiotic process, photodegradation, may degrade substrates in open-surface treatment areas. According to Martin Reinhard of Stanford University (personal communication 1990), reactions such as reductive dehalogenation occur abiotically as well as microbiologically, although the abiotic process can require hundreds of years to occur. Both of these processes are desirable in that they help to remove the target contaminant. They may also serve to decrease contaminant levels to levels which are too low. In this event, biological removal may become poor because of scarcity of substrate (Zaidi, Murakami, and Alexander 1988; Zaidi, Stucki, and Alexander 1988).

71. Competitive biological reactions may influence degradation rates without affecting the concentration of the target contaminant. Competition for a common resource such as oxygen may limit degradation by adversely affecting the growth or activity of the degrading microorganism, particularly if some step(s) in the degradation process requires oxygen (i.e., oxygenasedependent reactions). Competition between populations of degrading microorganisms and other soil microorganisms for other nutrients/resources may limit the growth of the degrader. Anything inhibiting the growth of degrading microorganisms will decrease the rate of degradation. 


\section{Other Factors Affecting Transformations}

72. Several other factors may influence the ability of microorganisms to degrade target contaminants in situ. These include structural properties of the contaminant (Alexander 1965); the physiological capabilities of the microbial communities present in the aquifer, groundwater, or vadose zone (Hickman et al. 1989); the interactions of mixtures of chemicals present (Scow, Schmidt, and Alexander 1989); the toxicity of the target contaminant (Hickman et al. 1989); and the length of adaptation periods required for degradation to be initiated (Aelion, Swindoll, and Pfaender 1987).

\section{Structural Properties of the Contaminant Molecule}

73. The contaminant itself may be refractory to biodegradation. Most natural products are biodegradable, even if the process occurs very slowly, because they are produced enzymatically. However, many man-made chemicals are not subject to normal enzymatic degradation or are broken down very slowly (Alexander 1965,1971$)$. The structure of these chemicals often prevents degradation by denying access to degrading enzymes, even if the contaminant is soluble; i.e., the chemistry of the contaminant stearically hinders the enzyme, preventing access to the core of the molecule. Dr. Alexander (personal communication 1990) gave the example of the effect of chlorination in decreasing the degradability of biphenyl. (This compound is quite degradable in the absence of chlorination.) Chlorination is thought to hinder degradation of DDT and other recalcitrant pesticides and tetrachlorodioxin (TCDD). Dr. Alexander indicated, however, that many compounds previously thought resistant can be degraded, given the proper conditions.

\section{Adequacy of the Microbial Community}

74. Even if a community of microorganisms can degrade a complex contaminant under certain conditions, the contaminant may not be degraded in situ. If a consortium of organisms having certain enzymatic capabilities must all be present in association with each other to bring about degradation, it is very possible that one or more components of the suite will be missing from the in situ environment. Addition of an organism having the requisite capability may 
be feasible; however, the chances of the organism surviving are not especially good (see section on addition of exotic and genetically engineered organisms).

\section{Mixed Chemicals and Degradation Kinetics}

75. According to Dr. Alexander (personal communication 1990), the kinetics of degradation of target contaminants differ in mixtures from what they would be alone. For example, when one organism is presented with two or more substrates that it can use, one substrate often is preferred over the other. The less preferred substrate may or may not be used. Simultaneous use of two substrates, when it occurs, is concentration-dependent (Schmidt and Alexander 1985), and the presence of a second substrate does not always preclude consumption of a target contaminant. For example, methylene chloride can be used as a secondary substrate by a strain of Pseudomonas sp. in the presence of acetate (LaPat-Polasko, McCarty, and Zehnder 1984). In another case, one microorganism may prefer one contaminant over other substrates present in a mixture. However, the remaining microorganisms present may grow rapidly on the other substrates, tying up nutrients required for growth by the organism degrading the contaminant.

76. A single target chemical in the presence of a mixed microbial community may also present problems ( $\mathrm{Dr}$. Alexander, personal communication 1990). For example, an organism using the target chemical may start to multiply, consuming the contaminant, but then be attacked by a predator or parasite. In this case, the target compound will be degraded poorly, if at all, because of the lack of a sufficient population of the degrading organism.

\section{Toxicity}

77. While species or consortia capable of using a target contaminant may be present, the compound may be sufficiently toxic to preclude consumption of all but minute amounts. Alternatively, some of the intermediate daughter products released during biodegradation may be toxic, either to the degrader species or to man. Finally, some of the daughter products may not be inherently toxic, but may undergo some abiotic reaction that produces a toxic substance. For example, some of the degradation products of amines can react in nature to form a highly toxic acylated derivative (Dr. Alexander, personal communication 1990). 
Adaptation

78. Adaptation is the process wherein a microbial community increases the degradation rate of a compound with exposure to that compound (Spain and Van Veld 1983). The lag period is the time during initial exposure in which the transformation rates of the chemical are slow (Lewis, Kolig, and Hodson 1986). The length of the 1 lag period reflects the degree of adaptation (Aamand et al. 1989). Several authors (Slater 1979; Spain, Pritchard, and Bourquin 1980; Aelion, Swindoll, and Pfaender 1987; Aamand et al. 1989) have considered the factors involved in the lag period, which is the period required for (a) induction of specific enzymes to carry out degradation; (b) exchange of genetic information brought about by plasmids; (c) development of new metabolic properties resulting from genetic changes; or (d) growth of the population of organisms already present that are capable of degrading the new substrate. Adaptation-related phenomena are related to other environmental factors considered in this report. For example, Lewis, Kolig, and Hodson (1986) have demonstrated that a shortage of nutrients will increase the lag period.

Population Levels of Degrading Microorganisms

79. As discussed previously, anything inhibiting the growth of degrading microorganisms decreases the rate of degradation. However, research conducted to determine the correlation of biodegradation rates to various measures of microbial activity in soil showed that, while the two types of data were directly related, correlation coefficients were generally low. Coefficients of determination showed that, at best, 87, 92, and 37 percent of the variability in subsurface biodegradation rates of methanol, phenol, and tertbutyl alcohol, respectively, could be attributed to differences in measures of bacterial density (Hickman and Novak 1989). This suggests that not all degradational activity is related to sheer microbial numbers.

Acclimation and Addition of Exotic and Genetically Engineered Species

80. As indicated in the section on adaptation, microorganisms may need to undergo adaptation (acclimation) to the degradational system. This is 
vitally important when considering addition of a biological treatment system for treatment of groundwater or vapors. If exotic species are added to the system, acclimation becomes an even more important factor.

81. There are many factors mitigating against the success of inoculation with exotic microorganisms, particularly with respect to the success of inoculation in enhancing the biodegradation of low levels of organic chemicals. This leads to a requirement to understand those environmental factors affecting activity or influencing the isolates that can grow rapidly at low substrate levels (Zaidi, Murakami, and Alexander 1988). The inoculated microorganism will also do poorly if there is no carbon source available or if the carbon source is only marginally suitable (Brunner, Sutherland, and Focht 1985). In addition, there is often only a very limited movement of inoculum in soil (Goldstein, Mallory, and Alexander 1985). Further, the toxins present in a pollutant-containing environment and/or grazing by protozoa suppress activity of inoculum (Goldstein, Mallory, and Alexander 1985). In a specific example, studies done by Bioremediation, Inc., using soils contaminated with polychlorinated biphenyls (PCBs) and others with PAHs indicated that while the white rot fungus Phanaerochaete chrysosporium can compete successfully in the environment, several problems arise. The enzyme activity of the organism is inhibited by many carbon substrates and elevated nitrogen levels. This means that substrates have to be limited. Also, the studies conducted to date involve mixing the soil with the substrate to achieve homogeneous dispersion of the target compounds (Emery and Kopecky 1989).

82. While there are many examples in the recent press of use of various types of preparations of microorganisms to degrade oil spills, the referred literature contains only limited documentation of such work. Westlake, Jobson, and Cook (1978) reported that seeding of oil-soaked plots with oildegrading bacteria had no effect on composition of recovered oil. Ralph Portier (personal communication 1990) claims to have several microbial amendments having good activity in spill situations. However, the most successful approach appears to involve fertilizing the existing spill location to improve growth of native oil-degrading species.

83. Use of genetically engineered microorganisms specific for degradation of individual contaminants for cleanup of waste sites presently is not an attractive option ( $\mathrm{Dr}$. Alexander, personal communication 1990), at least in the short-term, although microbial degradation of certain chloroaromatics is available now (Pierce et al. 1984; Boyle 1989). The whole issue of 
environmental compatibility of introduced species is wide open for future investigation.

\section{Degradation of Individual Groups of Contaminants}

84. The following sections consider the current state of understanding of the major groups of contaminants previously identified as important candidates for bioremediation efforts at military installations (see Part II). Only methyl ethyl ketone is not included. Degradation of the latter compound, while apparently not examined in environmental samples, is fairly well understood and a part of the aerobic degradation of other compounds as a prelude to acetate formation and the tricarboxylic acid cycle (for example, see Sperl and McKae (1980)).

\section{Microbial Degradation of Volatile Chlorinated Solvents}

85. A number of the chlorinated organic solvents are susceptible to biodegradation. While biodegradation of individual compounds may be favored by either aerobic or anaerobic conditions, several compounds may be degraded under both conditions. Our ability to demonstrate biodegradation in the laboratory, however, does not mean that breakdown will occur readily in the field. Some of these compounds appear to persist for extended periods in the soil.

Aerobic Degradation

86. At present, we know that aerobic degradation of volatile chlorinated organic solvents can be brought about through the cometabolic activities of various species of methane-oxidizing bacteria (methylotrophs) (Wilson and Wilson 1985). Degradation is brought about by methane monooxygenase, an enzyme that is able to oxidize several of the halogenated organic solvents; e.g., trichloroethene, vinyl chloride, dichloromethane, dibromomethane, and dilodomethane (Klecka 1982; Patel 1984; Fogel, Taddeo, and Fogel 1986; Little et al. 1988; Henson et al. 1988). The mechanism apparently involves epoxidation of the double bond to form unstable intermediates that spontaneously degrade to produce simple molecules. In this reaction sequence, trichloroethene is cleaved to yield chloride ions, carbon dioxide, formate, and glyoxylic acid (Fogel, Taddeo, and Fogel 1986) or glyoxylic acid and 
dichloroacetate (Little et al. 1988). A similar sequence may be responsible for the degradation of vinyl chloride, which is oxidized first to chloroethylene epoxide and then spontaneously rearranges to produce chloroacetaldehyde. The latter compound subsequently hydrolyzes to glycolaldehyde (Fogel, Taddeo, and Fogel 1986).

87. The cleavage products of methane oxidase activity may be further metabolized by the methylotrophs themselves or by other organisms in the community (Fogel, Taddeo, and Fogel 1986). Alternatively, degradational activity may be a purely cometabolic process, with the responsible methylotrophs deriving little or no direct benefit from the process (Morgan and Watkinson 1989). Other investigators have indicated that aerobic degradation of trichloroethene may also produce dichloroethylene, vinylidine chloride, and, possibly, chloroform (Fliermans et al. 1988). The exact mechanism of trichloroethene degradation has not been specified.

88. An alternate pathway for aerobic degradation of trichloroethene has been described for microorganisms that use an aromatic biodegradative pathway with dioxygenase activity (Nelson et al. 1987; Nelson, Montgomery, and Pritchard 1988; Wackett and Gibson 1988). Degradation of low levels of trichloroethene by heterotrophic microorganisms has been reported by Nelson et al. (1987). However, for mineralization to proceed to form $\mathrm{CO}_{2}$, aromatic compounds such as phenol, toluene, o-cresol, or $m$-cresol, were required to support both the growth and enzymatic activity of the trichloroethene-degrading organisms.

89. Various strains of Pseudomonas (LaPat-Polasko, McCarty, and Zehnder 1984), particularly P. putida (Nelson, Montgomery, and Pritchard 1988; Wackett and Gibson 1988), are able to carry out the degradation of various halogenated organic solvents under aerobic conditions. Other active aerobic microorganisms include strain G4 of Nelson et al. (1987), Methylosinus trichosporium (Wackett and Gibson 1988), and Methylmonas MM2 (Grbic-Galic 1990), as well as aerobic microbial communities (Henson et al. 1988). Aerobic populations capable of degrading halogenated organic solvents have been found in samples of groundwater (Bouwer, Rittmann, and McCarty 1981), sandy soil (wilson and Wilson 1985), sewage treatment plants (Tabak et al. 1981), sediments (Fogel, Taddeo, and Fogel 1986) and "subsurface sediments" (Fliermans et al. 1988), and in soll from Moffet field (Grbic-Galic et al. 1990). 
90. Anaerobic degradation of halogenated organic solvents can be supported by several-different processes. Microbial populations isolated from sewage treatment plants have been observed to transform several halogenated organic compounds, including carbon tetrachloride, dichloromethane, and brominated trihalomethanes, under denitrifying conditions (Bouwer and McCarty 1983a). While the exact mechanism for this activity is not known, the appearance of chloroform in cultures degrading carbon tetrachloride suggest reductive dechlorination as one possible mechanism. However, chloroform was never detected as a reaction intermediate in batch and continuous flow studies on transformability of halogenated organic compounds under methanogenic conditions. Dehalogenation is also possible under sulfate-reducing conditions, where tribromomethane (bromoform), carbon tetrachloride, bromodichloromethane, and hexachloroethane are attacked (Bouwer and Wright 1988), although some results contradict this (Suflita, Gibson, and Beeman 1988).

91. Reductive dehalogenation is also known to occur under methanogenic conditions, and this may be followed by at least partial mineralization of the products to $\mathrm{CO}_{2}$ (Vogel and McCarty 1985). In reductive dehalogenation, the chlorinated compound accepts electrons and is reduced to the corresponding hydrogenated compound, while the chlorine is released as chloride (Milde, Nerger, and Mergler 1988). Electron donors for this process may be provided by other contaminants present or by natural sources of carbon in the soil. A pathway for reductive dehalogenation of tetrachloroethene was proposed by Vogel and McCarty (1985) and subsequently modified by others (Milde, Nerger, and Mergler 1988). This process can be highly effective in soil and may result in rapid dehalogenation of highly chlorinated compounds that are only slowly degraded under aerobic conditions. Reductive dechlorination has been shown to form trichloroethylene from tetrachloroethane and 1,2,2-trichloroethane from 1,1,2,2-tetrachloroethane (Bouwer and McCarty 1983 a,b; Vogel and McCarty 1985). Other compounds degraded include 1,1,2-trichloroethylene (Barrio-Lage, Parsons, and Lorenzo 1988; Vogel and McCarty 1987) and tetrachloroethylene (Fathepure and Boyd 1988). It is important to note that the products of reductive dehalogenation may be completely dehalogenated or only partially metabolized, resulting in a risk of persistent, undesirable metabolites, such as vinyl chloride from tetrachloroethene. However, vinyl chloride 
will degrade to methane and ethylene under the proper environmental conditions.

92. Little is known about the organisms carrying out anaerobic degradation of halogenated organic compounds. The concern here has been with anaerobic degradation as a process, rather than with specific microorganisms.

Milde, Nerger, and Mergler (1988) listed the following organisms as being active in the degradation of tetrachloroethene: Clostridium cadaveris, Clostridium limnosum, Pseudomonas maltophilia, Pseudomonas fluorescens, Proteus vulgaris, Enterobacter cloacae, Escherichia coli, Pseudomonas aeroginosa, plus a variety of gram positive and gram negative bacteria. Trichloroethene degrading populations have been observed in continuous-flow fixed-film methanogenic columns (Vogel and McCarty 1985), batch cultures and continuous-flow methanogenic fixed-film laboratory-scale columns (Bouwer and McCarty 1983b; Vogel and McCarty 1985), active denitrifying bacterial cultures obtained by seeding with sewage effluent (Bouwer and McCarty 1983a), groundwater (Milde, Nerger, and Mergler 1988), and methanogenic mixed cultures grown in laboratory-scale digestors fed with waste activated sludge (Bouwer, Rittman, and McCarty 1981).

\section{Discussion and Conclusions}

93. Based on the above 1iterature, it is clear that the pathways for biodegradation of volatile chlorinated organic solvents are fairly well understood. The feasibility of using microbiological degradation for cleanup of soil and groundwater contaminated with halogenated organic compounds was considered in detail by Morgan and Watkinson (1989). They noted that these compounds were "prime targets" for bioremediation because of their frequent occurrence in the environment and the fact that many of the commonly used compounds are degradable. Nonetheless, the chlorinated solvents as a group do not appear to be readily available and cannot be used by microorganisms as a primary source of carbon. Their review of technologies available for bioremediation of contaminated soils and groundwaters examined both in situ and extraction-treatment techniques. Based on the information presented above and also from Morgan and Watkinson's article, the individual factors limiting or preventing biodegradation under in situ conditions will be highly sitespecific. Clearly, research emphasis should be placed on procedures that will assess the need for cometabolites and the limiting factors present at a given 
site to determine which factors are associated with the persistence of these otherwise biodegradable compounds.

\section{Microbial Degradation of Petroleum Hydrocarbons}

94. Petroleum is a complex heterogenous mixture of organic compounds that vary from easily degraded to recalcitrant. From the hundreds of possible compounds present in petroleum, several classes can be broken out, based on commonality of structure. According to Atlas (1981), the mixture can be broken down into a saturated or aliphatic fraction, an aromatic fraction, and an asphaltic or polar fraction. Saturated hydrocarbons include the n-alkanes (linear or straight-chain molecules), branched alkanes, and cycloalkanes (naphthenes). Aromatic compounds are ring-containing compounds based on benzene as the parent type and may be considered as derivatives of benzene. Some polar compounds are highly complex structures that are difficult to analyze.

95. Most of the petroleum hydrocarbons of military importance are fuels. Fuels are complex mixtures of petroleum hydrocarbons, but are comprised of groups of compounds within a range of molecular weights. The fuels most commonly used in the Army at present are gasoline and diesel oil, although heating oil will also be important for military installations located in the temperate regions. The following percent analysis for gasoline, heating oil, and diesel oils is based on that given by Song, Wang, and Bartha (1990) in a recent bioremediation study.

\begin{tabular}{|c|c|c|c|c|}
\hline Fue1 & Saturates & Aromatics & Polar Compounds & $\begin{array}{c}\text { Carbon } \\
\text { Range }\end{array}$ \\
\hline Gasoline & & Not analyzed & & $\mathrm{C} 6-\mathrm{C} 11$ \\
\hline $\begin{array}{l}\text { Heating } \\
\text { Oil }\end{array}$ & 62.5 & 32.9 & 1.9 & $C 9-\mathrm{C} 22$ \\
\hline $\begin{array}{c}\text { Diesel } \\
\text { Oil }\end{array}$ & 53.7 & 45.0 & 1.3 & $\mathrm{C} 9-\mathrm{C} 23$ \\
\hline
\end{tabular}

96. Based on this analysis, fuels are made up of different kinds and amounts of hydrocarbons, and the average number of carbon atoms in each hydrocarbon also varies.

97. Several studies have been conducted to determine the metabolic pathways for biodegradation of petroleum hydrocarbons. These include two recent reviews on the subject (Atlas 1981, 1984). However, few reports on 
petroleum hydrocarbon degradation in soils and soil enrichments appear in comparison to aquatic systems. Most of the attention has focussed on the aquatic environment as a result of the severe problems encountered with spill containment in water (Jones 1977; Bossert and Bartha 1984). However, spills on land can also be a problem because of lateral and vertical spreading and potential access to the water table (Atlas 1981).

Degradation of Major Groups of Petroleum Hydrocarbon Components

98. A large body of information exists on the biodegradation of components of the various groups of compounds present in fuel. Relevant portions of this information will be summarized here. Details on degradation of each of the classes are available in the references cited.

99. The $n$-alkanes are thought to be the most readily degradable portion of a petroleum mixture (Atlas 1981), and biodegradation of the n-alkanes has been demonstrated for compounds having molecular weights ranging up to 44 carbon atoms (Haines and Alexander 1974). The following summary of the microbial metabolism of straight-chain and branched chain alkanes is based on a review by Singer and Finnerty (1984). Both short and long-chain alkanes are oxidized through one of three mechanisms. Degradation of $n$-alkanes usually occurs via sequential monoterminal alkane oxidation (oxidation of one end of the chain) mediated by mono- or dioxygenases to the corresponding alcohol, aldehyde, and then fatty acid, and then to acetate by beta oxidation. Degradation of acetate can proceed to produce shorter fatty acids and, eventually, $\mathrm{CO}_{2}$. Some organisms can carry out diterminal or omega oxidation of both ends of an alkane, while still other organisms perform subterminal oxidation (oxidation near the end of the molecule). Subterminal oxidation can also occur, forming a secondary alcohol and then a ketone; but this is apparently a minor degradative pathway. Branched chain alkane degradation also occurs; however, the metabolic process becomes more complicated because the presence of chains can sometimes hinder microbial oxidation of the molecule. Thus, 2-methyl branched compounds are normally capable of supporting good growth, while 3-methyl branched compounds are attacked by very few microorganisms.

100. Cycloalkanes are major components of petroleum that are notably resistant to microbial degradation; and complex substances, such as the tripentacyclic compounds, are extremely persistent (Atlas 1981). The following summary on metabolism of cycloalkanes, otherwise known as alicyclic or 
cycloparaffinic compounds, is based on the reviews of Perry (1984) and Trudgill (1984). Examples of representative cycloalkanes present in gasoline include cyclopentane, cyclohexane, methylcyclopentane, and trimethylcyclopentane (Perry 1984). These compounds have been present on earth for millions of years, are actively produced by both plants and bacteria, and are components of waxes, oils, and secondary metabolites in plants, microbial lipids, and insect secretions (Trudgil1 1984). Based on this, Perry indicates that it is surprising that investigators have failed to demonstrate the presence of large numbers of cycloalkane-utilizing microorganisms. Trudgill indicates that there is a general consensus that alicyclic hydrocarbons are more resistant to microbial degradation than most other hydrocarbons. Trudgill (1984) and Perry (1984) speculate that this may be due to one or more of several different factors, including the broad specificity of monooxygenase enzymes that convert cycloalkanes to cycloalkanones. Consequently, the cycloalkanes may be metabolized rapidly through cooxidation and commensalism (the process wherein the growth of one organism promotes the growth of a second species, but the growth of the latter does not affect the former (Linton and Drozd 1982)); thus the compounds may not have ever accumulated in levels great enough to select for individual organisms capable of using them. Alternatively, certain cycloalkanes may be sufficiently complex as to preclude utilization by pure cultures of organisms. Based on this understanding, several workers have sought and found indications that biodegradation of recalcitrant cycloparaffinic molecules occurs through cooxidation and commensalism.

101. Gibson and Subramanian (1984), Cerniglia (1984), and others (Edwards 1983; Bossert and Bartha 1986) have summarized the results of recent investigations of aromatic hydrocarbon degradation. The light aromatic hydrocarbons undergo both evaporation and microbial degradation while in the dissolved state (Kappeler and Wuhrmann 1978). The fate of PAHs is particularly important because many of them are both toxic and carcinogenic and occur naturally, perhaps even forming important structural units of humic substances (Martin, Haider, and Bondiett 1972).

102. Cerniglia has noted that many bacteria, fungi, and algae are enzymatically capable of metabolizing aromatic compounds, but the hydroxylation mechanisms among these groups of organisms are fundamentally different. Moreover, most of the studies of microbial metabolism of aromatic hydrocarbons have been carried out using pure cultures and/or purified enzymes. While this work provides a good understanding of the ability of microorganisms to degrade 
these compounds and the mechanisms involved in the degradation, little is known about the environmental conditions required for degradation. At the time Cerniglia's article was written, the degradation pathways that had been worked out generally required aerobic conditions to proceed. He and Gibson and Subramanian (1984) have summarized literature describing pathways for a vast array of compounds, including benzene, naphthalene, phenanthrene, anthracene, benzo[a]pyrene, benz [a]anthracene, 7-methylbenz [a] anthracene, and 3-methylcholanthracene. According to these authors, many of the pathways for the more complex compounds require participation by several organisms, although there are exceptions. For example, Heitkamp, Franklin, and Cerniglia (1988) have recently isolated a Mycobacterium that can completely mineralize pyrene. Bacteria oxygenate compounds aerobically using a mono- or dioxygenase to form dihydrodiols having a cis-configuration. Fungi use a mono-oxygenase to form trans-dihydrodiols. Later work by Mihelcic and Luthy (1988), for example, has shown that several PAHs degrade readily under denitrifying conditions. This process is carried out by bacteria, but the details of the pathways have not been established. Recent research has shown that mono- and polynuclear aromatic hydrocarbons can also be transformed by anaerobic microbial communities under denitrifying, fermentative (including methanogenic) and in some cases, sulfate-reducing conditions (Grbic-Galic 1990). However, very little work has been done to document the importance of anaerobic degradation of these compounds (Young 1984; Grbic-Galic 1989, 1990).

103. Atlas (1981) indicated that the metabolic pathways for degradation of the asphaltic or polar compounds are not very well understood because of their complex structure. He also suggested that advances in determining degradative pathways for these compounds will depend on improvements in analytical procedures.

104. The rate of microbial degradation of individual hydrocarbons in general and complex mixtures of hydrocarbons, such as fuels in particular, is considerably reduced because of the low solubility of many of the components. Microorganisms using hydrocarbons as substrates produce a number of different surfactants and emulsifiers, some of which are derived from the hydrocarbons being degraded (Rosenberg et a1. 1979; Desai, Patel, and Desai 1988; Zajick and Mahomedy 1984). Surfactants and bioemulsifiers undoubtedly provide the generating microorganism with the means to more readily obtain insoluble carbon sources (Desai, Patel, and Desai 1988). 
105. Bossert and Bartha (1984) have summarized the structure, degradability, and toxicity of hydrocarbons in soil based on the reviews of Bartha and Atlas (1977), Atlas (1981), and the National Academy of Sciences (1975). According to this information, petroleum components including the $n$-alkanes, the $n$-alkylaromatics, and the aromatic compounds in the $C_{10}-C_{22}$ range are the least toxic, but the most biodegradable. While the n-alkanes, alkylaromatics, and aromatic hydrocarbons in the $\mathrm{C}_{5}-\mathrm{C}_{9}$ range are degradable at low concentrations, they are highly toxic and are removed primarily by volatilization. The branched alkanes in the $\mathrm{C}_{10}-\mathrm{C}_{22}$ range are less biodegradable because the branching hinders microbial attack. As indicated above, biodegradation of the cycloalkanes requires some sort of interaction by two or more microbial species. The complex aromatic and cycloparaffinic compounds having four or more condensed rings are also extremely resistant to degradation, with degradation requiring the participation by several species of microorganisms (Cerniglia 1984; Gibson and Subramanian 1984).

\section{- Microorganisms Degrading Petroleum Hydrocarbons}

106. Much work has been done with individual microorganisms that degrade one or a variety of petroleum hydrocarbons. A list of these organisms is too lengthy to enumerate in detail here (Atlas 1981 and the other reviews cited above). Representative organisms degrading alkanes include the bacteria Pseudomonas aeruginosa, Pseudomonas putida, various species of Acinetobacter, and the yeast Candida (Singer and Fennerty 1984). Organisms active on cyclic alkanes include several species of Pseudomonas, Nocardia, and Acinetobacter (Perry 1984). One of the most well-known organisms active on aromatic hydrocarbons is the fungus Cunninghamella elegans; although there are many bacteria capable of degrading aromatic hydrocarbon, algae are also active on this group of compounds (Cerniglia 1984). An understanding of the environmental characteristics and microbial ecology of specific microorganisms that are active on petroleum hydrocarbons in nature is very limited, since past work has not involved extensive use of environmental samples.

\section{Degradation of Fuels in Soils}

107. Despite the wealth of information on microbial degradation of individual petroleum hydrocarbon components and the pathways responsible for 
degradation of these compounds, considerably less information exists in the open literature on the rate and extent of fuel degradation in soil. Nonetheless, there is some information available (in addition to the reviews of Atlas (1981), (1984), see Jones (1977); Westlake, Jobson, and Cook (1978); Dibble and Bartha (1979); Fedorak and Westlake 1981; Aamand et al. (1989); and Carberry and Lee (1990)). There are also numerous reports in the grey literature-for example, the work of Ridgeway et al. (1988). Soils contaminated by petroleum fuel spills are considered hazardous wastes, and the disposal of large volumes of contaminated soil by burial in secure sites or incineration is very expensive. By contrast, land treatment of oily refinery sludges has been conducted for years with generally good results (Song, Wang, and Bartha 1990).

108. Initial steps of hydrocarbon degradation are apparently either oxygen-dependent or proceed much more rapidly under aerobic conditions (Bossert and Bartha 1984). While Mihelcic and Luthy (1988) were able to demonstrate anaerobic degradation of some PAHs under denitrifying conditions in soil slurries, other authors have not been as successful. Ward and Brock (1978) and DeLaune, Hambrick, and Patrick (1980) have indicated that anaerobic degradation of petroleum hydrocarbons is either negligible or nonexistent. Hambrick, Delaune, and Patrick (1980) examined degradation of radiolabeled hydrocarbons in estuarine sediment and found up to 15 percent degradation at redox potentials of $-220 \mathrm{mV}$ and $\mathrm{pH} 8.0$. Biodegradation, however, reached considerably higher levels at more positive redox potentials. Bossert and Bartha (1984) expressed reservations about the results of this work because measurements were made of volatile $14 \mathrm{CO}_{2}$ trap results only, and other volatile compounds were not assessed.

109. Dibble and Bartha (1979), Atlas (1981), and Bossert and Atlas (1984) have considered the environmental factors influencing biodegradation of petroleum hydrocarbons in soils. Soils do not always provide the necessary combinations of nutrients, moisture, $\mathrm{pH}$, and temperature for optimal microbial activity. Too much moisture interferes with oxygen movement into the soil, inhibiting aerobic degradation. In addition, the growth of fungi, some of the most effective agents of degradation, can be limited by too much water. While sulfate can serve as an alternate electron acceptor for anaerobic degradation, many soils do not have high sulfate levels. Unlike many other situations on military installations where organic compounds are found at very low levels, petroleum hydrocarbon spills can inundate the soil with high levels of 
carbon-rich compounds. Under these circumstances, nitrogen and phosphorus can easily become limiting, as has been evidenced by the vast improvement in degradation brought about by addition of fertilizers (Dibble and Bartha 1979).

110. Several reports in the literature deal with fuel spills and/or are focused on degradation of one or more components of more complex fuel mixtures (Ridgeway et al. 1988; Wilson et al. 1988; Aamand et al. 1989; Ostendorf 1990). Reportedly, Song, Wang, and Bartha (1990) investigated a bioremediation approach for cost-effective land treatment for five different fuels: gasoline, jet fuel, heating oil, diesel oil, and bunker $C$. They found that biodegradation makes only a modest contribution to the disappearance of gasoline from soil, while volatilization can play a major role. A similar role for volatilization was reported by Ostendorf (1990), who indicated that volatilization may account for 30 percent of the original gasoline spilled at the US Coast Guard Air Station, Traverse City, MI. Song, Wong, and Bartha (1990) also found that bunker $\mathrm{C}$ was structurally recalcitrant (resisted degradation), with nearly 80 percent persisting for a year after initiating treatment. The three medium distillates, jet fuel, heating oil, and diesel oil, increased in persistence in that order, but responded well to bioremediation treatment. Song and Bartha (1990) examined the effects of jet fuel spills on soil microbial community structure, and function and disappearance from surface soils was much more rapid than from poorly aerated subsurface soil. Jones (1977) studied the effects of kerosene spills on soil and found very little change in the polluted sites. He speculated that decomposition was reduced because of hydrocarbons and fatty end products by preventing gas exchange at the soil surface. The studies of Song, Wong, and Bartha, Song and Bartha, and Jones dealt with fuel spills restricted to the upper half meter or less of the soil surface. Conditions here are much different from those in the deeper soils that will be the focus of most in situ work.

\section{Discussion and Conclusions}

111. A large body of information exists on the microbial degradation of individual compounds in petroleum hydrocarbon mixtures, such as crude oil and fuels. The mechanisms and pathways for many of these compounds have been established, and many of the responsible organisms have been identified. By contrast, only a limited amount of information on degradation of these 
mixtures in soil is available, although a substantial amount of information on fuel and petroleum spills in water can be found.

112. The information available on individual petroleum components indicates that under the proper circumstances, the linear alkanes and aromatic hydrocarbons degrade fairly readily, with the cyclic alkanes being considerably more resistant to degradation. Most of the known pathways of degradation require the presence of oxygen. However, recent research indicates that for the aromatic hydrocarbons, at least, oxygen may be supplied by nitrate, sulfate, or even iron oxyhydroxides. It is unclear at this point whether or not anaerobic degradation supported by these alternate electron acceptors is capable of removing enough hydrocarbons to achieve acceptable levels of bioremediation in situ.

113. It appears that while there is a potential for petroleum hydrocarbon removal under anaerobic conditions in situ, sources of oxygen, the necessary nutrients, and the requisite microorganisms must all be present in the contaminated soil to obtain the most complete degradation of petroleum hydrocarbons (Wilson et al. 1985; Aamand et al. 1989; Grbic-Galic 1990). This may not always be enough, however. In a recent study assessing the potential for in situ biotreatment of hydrocarbon-contaminated soils, Morgan and Watkinson (1990) found that while available data indicated that biotreatment of a gasoline and lubricating oil-contaminated site was viable, the addition of nutrients inhibited mineralization in the soils. For these reasons, research on in situ biotreatment of saturated soils contaminated with petroleum hydrocarbons should focus on development of procedures to determine the best means to provide oxygen to the degrading microflora and on the assessment of limiting factors present at a given site.

\section{Degradation of Benzene, Toluene, Ethylbenzene, and Xylene}

114. Benzene, toluene, ethylbenzene, and xylene (BTEX) are homoaromatic hydrocarbons derived from benzene or other petroleum products or formed biologically by microorganisms during transformation of aromatic lignin or other compounds and from leakage of underground storage tanks containing gasoline (Healy, Young, and Reinhard 1980; Grbic-Galic and Young 1985). BTEX compounds are common soil and groundwater pollutants because of their widespread use in the chemical and manufacturing industries as solvents and in synthesis of plastics, paints, and other chemicals (Cerniglia 1984; Grbic-Galic 1990). 
General information on aromatic hydrocarbons and their microbial degradation can be found in the review section on degradation of petroleum hydrocarbons.

Aerobic Oxidation

115. Microbial oxidation of benzene has been observed by various investigators (McFarlane et al. 1981) and reviewed by Cerniglia (1984) and by Gibson and Subramanian (1984). The aerobic pathway involves utilization of molecular oxygen in a dioxygenase-catalyzed reaction to produce a cis-1,2dihydroxy-1,2-dihydrobenzene (some investigators indicate that this compound is really a cis-benzene dihydrodiol). This compound is then enzymatically converted to catechol. Catechol is subsequently oxidized by either the ortho or the meta fission pathway, both of which feed directly into major biochemical pathways in microorganisms.

116. As reviewed by Cerniglia (1984), toluene is initially oxidized aerobically at the methyl group to form benzoic acid or on the benzene ring to form a dihydrodiol having a cis-configuration. The benzoic acid is then susceptible to oxidation to catechol and subsequent ring fission as described for benzene, while the dihydroxy-dihydrotoluene first undergoes conversion to the methylcatechol before ring fission can occur. Davey and Gibson (1974) demonstrated that bacteria capable of utilizing $m$ - and $p$-xylene oxidized these compounds to the corresponding toluates through sequential oxidation of a methyl group to the level of an alcohol, then an aldehyde, and finally the acid.

117. According to Gibson and Subramanian (1984), ethylbenzene is oxidized to a cis-dihydrodiol by Pseudomonas putida. The reactions beyond this point cleaves the ring.

Anaerobic Degradation

118. The anaerobic degradation of these compounds was unknown until recently (Grbic-Galic 1990). This was in large part due to the belief that the buildup of petroleum hydrocarbon deposits in anaerobic subsurface areas resulted from the inability of microorganisms to utilize these compounds in the absence of oxygen. The anaerobic degradation pathways of benzene and toluene differ substantially from the aerobic pathways. According to GrbicGalic (1990), there appear to be three degradation pathways for toluene 
degradation occurring in complex methanogenic cultures. The initial step in each pathway involves addition of oxygen obtained from water. The principal pathway involves ring oxidation of toluene at the para position to produce $p$ cresol. A second pathway involves oxidation of the methyl group to form benzyl alcohol, which is subsequently oxidized to benzaldehyde and benzoic acid. A minor pathway utilizes ring fission at the ortho position to produce $o$-cresol. Once these oxidized intermediates have been formed, they are further transformed via established pathways for anaerobic degradation of aromatic alcohols and acids. Benzene is also initially oxidized with oxygen derived from water and can be completely degraded to $\mathrm{CO}_{2}$ and $\mathrm{CH}_{4}$. The pathway for ethylbenzene degradation is not specified, but would probably be similar to the pathway for toluene--i.e., first the terminal methyl group on the molecule would be attacked and oxidized to alcohol, aldehyde, acid, and then released as $\mathrm{CO}_{2}$, after which the new toluene molecule would be degraded as described above.

119. A number of investigations have been conducted to examine the metabolism of homocyclic aromatic compounds under anaerobic conditions (see Berry, Francis, and Bollag (1987) and Grbic-Galic (1990)). Other investigators have demonstrated anaerobic degradation of toluene, benzene, ethylbenzene, and xylene using methanogenic materials from an aquifer (Wilson, Smith, and Rees 1986). Kuhn et al. (1985) demonstrated microbial transformations of substituted benzenes during infiltration of river water to groundwater. Both dimethyl and dichlorobenzenes were transformed aerobically, while the dimethylbenzenes were degraded anaerobically by denitrifying bacteria. Zeyer, Kuhn, and Schwarzenbach (1986) showed that nitrate could be utilized as an electron acceptor in place of $\mathrm{O}_{2}$, and that microorganisms adapted to growth on $m$-xylene were also able to degrade toluene under denitrifying conditions. Lovely and Lonergan (1990) found that iron reduction supported removal of toluene, phenol, and $p$-cresol and proposed a pathway for the degradation process. Thomas et al. (1990) found that toluene, ethylbenzene, and $m$-xylene were removed anaerobically from core samples taken from subsurface materials contaminated with gasoline.

\section{Microorganisms Degrading BTEX Compounds}

120. As indicated in the section on petroleum hydrocarbon degradation, many organisms are capable of degrading aromatic hydrocarbons, including the 
BTEX compounds. Aerobic microorganisms especially active on BTEX compounds are strains of Pseudomonas and Nocardia (Omori and Yamada 1979; Cerniglia 1984; Gibson and Subramanian 1984; Inoue and Horikoshi 1989). Pseudomonas putida is a particularly interesting organism in that some strains produce a toluene dioxygenase that is also active against trichloroethylene (Wackett and Gibson 1988). Anaerobic microorganisms degrading BTEX compounds include methanogenic consortia (Grbic-Galic and Voge1 1987; Grbic-Galic 1990), denitrifiers (Kuhn et al. 1985; Major, Mayfield, and Barker 1987), or sulfate reducers (Cord-Ruwisck and Garcia 1985; Berry, Francis, and Bollag 1987). A recent report indicates that anaerobic degradation of some BTEX compounds can also be supported through the use of ferric iron as an alternate electron acceptor by a pure culture of GS-15 (Lovely and Lonergan 1990).

\section{Discussion and Conclusions}

121. Just as for the other aromatic hydrocarbons, the degradation of the BTEX compounds is influenced by a number of environmental factors, and these will not be repeated here. Of the many environmental variables, perhaps the most important is the presence of oxygen. Although many pathways have been shown to occur in the presence of electron acceptors (see reviews cited above), the BTEX compounds disappear more rapidly in the presence of oxygen. It is important to note, however, that other factors in addition to the lack of oxygen may affect the degradation of hydrocarbons (Alexander 1965; Berry, Francis, and Bollag 1987). If alternate electron acceptors are utilized for anaerobic degradation, then the concentration of these compounds becomes important, assuming that suitable microorganisms are present. Investigations by Thomas et al. (1990) indicate that enhancement of in situ biodegradation for removal of BTEX compounds does work, but that degradation is slow. While microbial numbers and activity in the biostimulated zone remained enhanced for at least 2 years after the in situ process had been terminated, residual hydrocarbons still remained. 
TNT

122. Until recently, mineralization of TNT by microorganisms had not been demonstrated. The only microbial degradation described in the literature was transformations of the nitro moieties to amino and hydroxylamino groups or coupling of rings to azo or azoxy compounds (Carpenter et al. 1978; Hoffsommer et al. 1978; Kaplan and Kaplan 1982; Kaplan et al. 1985; McCormick, Cornell, and Kaplan 1978; Naumova, Amerkhanova, and Shaykhutdinov 1979). Kaplan and Kaplan (1982) identified the following products: 2-amino-4, 6-dinitrotoluene, 4-amino-2,6-dinitrotoluene, 2',4,6',6-tetranitro-2,4'-azoxytoluene, 2, 2',6, 6'-tetranitro-4,4',-azoxytoluene, 2,4-diamino-6-nitrotoluene, and 2,6-diamino4-nitrotoluene. They also proposed a biotransformation scheme which included three products, 4-hydroxylamino-2, 6-dinitrotoluene, 2-hydroxylamino-4, 6dinitrotoluene, and 4,4',6,6'-tetranitro-2,2'-azoxytoluene, which they were unable to identify in their extracts. The microorganism(s) active in TNT transformation was not identified.

123. Naumova, Selivanovskaya, and Cherepneva (1988) report a similar transformation pathway by Pseudomonas fluorescens. However, they suggest that the formation of the monoamino compounds and the subsequent formation of 2,4dinitrotoluene are favored under anaerobic conditions. Furthermore, phloroglucinol and pyrogallol accumulated in cultures provided with 2,4dinitrotoluene as sole carbon source (Selivanovskaya et al. 1986), suggesting that these compounds are intermediates in further transformation reactions. This reaction was more favorable under aerobic conditions. Formation of these intermediates leads to ring cleavage and, ultimately, to mineralization of TNT (Naumova, Selivanovskaya, and Mingatina 1988).

124. A group of Chinese researchers ( $\mathrm{Li}$, Yin, and Yang 1987) report TNT degradation by a strain of Citrobacter freundii under aerobic and microaerophilic conditions. The pathway and the enzymes involved are not described in the English translation of the abstract (entire report in English not yet available); however, several TNT analogues are reported to inhibit the reactions; m-dinitrobenzene, 2,4-dinitrophyno1, and p-nitrobenzene.

125. Three Americans (Fernando, Bumpus, and Aust 1990) report TNT degradation by the white rot fungus, Phanerochaete chrysosporium. Using ${ }^{14} \mathrm{C}-$ labeled TNT, the authors observed 18 percent mineralization of $10,000 \mathrm{mg}$ 
TNT $/ \mathrm{kg}$ of soil and almost 20 percent mineralization of $100 \mathrm{mg} \mathrm{TNT} / \mathrm{kg}$ of water to ${ }^{14} \mathrm{CO}_{2}$ in 90 days. Untransformed TNT remaining was about 15 percent. Identification of enzymes and intermediates is incomplete.

126. In addition to these findings, two independent American research groups report mineralization of TNT by unidentified consortia of environmental isolates (Unkefer et al. 1989; Montemagno and Irvine 1989). While neither of these researchers has yet published details of his work, personal communications suggest that the degradation pathway observed by Unkefer may be similar to that reported by Selivanovskaya and associates.

127. To implement in situ bioremediation of TNT-contaminated soils or groundwater, a more complete understanding of the microbial degradation processes is needed. Although a mineralization pathway for TNT has been described, the accumulation rate, environmental effects, and ultimate fate of intermediates are not known. It is likely that some of the amino compounds will be oxylated, as demonstrated by, for example, Hallas and Alexander (1983). Environmental factors required for optimization of degradation rate are incompletely understood, and how ubiquitous TNT-degrading microorganisms are at contaminated sites is unclear. Effective operational strategies for in situ bioremediation cannot be developed without additional information on microbial degradation of TNT.

\section{RDX}

128. Degradation of $\mathrm{RDX}$ in sediments and mineralization to $\mathrm{CO}_{2}$ under anaerobic conditions were demonstrated by Spanggord et al. (1980) and Sikka et al. (1980), respectively. However, these researchers were unable to identify the products of degradation. McCormick, Cornell, and Kaplan (1981) identified the products of microbial degradation to include hexahydro-1-nitroso-3,5-dinitro-1,3,5-triazine, hexahydro-1,3-dinitroso-5-nitro-1,3,5-triazine, hexahydro1,3,5-trinitroso-1,3,5-triazine, hydrazine, 1,1-dimethylhydrazine, 1,2dimethylhydrazine, formaldehyde, and methanol. Some of these products may be carcinogens, especially the $\mathrm{N}$-nitroso compounds. McCormick, Corne1l, and Kaplan (1981) also proposed a biodegradation scheme which accounted for all of the observed products. McCormick, Corne11, and Kaplan demonstrated conclusively that biodegradation proceeds only under anaerobic conditions. The authors suggest that remediation efforts include an initial anaerobic step to reduce $\mathrm{RDX}$ wastes to hydrazines and methanol, followed by an aerobic step to 
oxidize the methanol. The biodegradability of hydrazines is unknown, but their mutagenicity and carcinogenicity are well established (Fiala 1977;

Greenhouse 1976). Therefore, biodegradability of hydrazines must be determined before implementing a technology that would generate them. 


\section{Introduction}

129. Adsorption and desorption processes often exert a dramatic effect upon movement of contaminants through vadose and aquifer soils (Freeze and Cherry 1979; Thibodeaux 1979; Curtis, Roberts, and Reinhard 1986; Brusseau and Rao 1989; Mercer, Skipp, and Giffin 1990; Travis and Doty 1990). Interphase transfer of contaminants between soil solids and pore water is potentially limited by diffusive mass-transfer resistance (Rao et al. 1980; Liu and Weber 1981; Nkedi-Kizza et al. 1982; Aguwa et al. 1984; Roberts, Cornel, and Summers 1985; McKay, McKee, and Walters 1987; Roberts et al. 1987). Slow rates of interphase transfer can greatly limit the effectiveness of pump-and-treat remediation by preventing sufficient and timely removal of contaminants from soil (Mercer, Skipp, and Giffin 1990; Travis and Doty 1990). Slow desorption can also restrict microbial access to contaminants during in situ bioremediation. Contaminants bound to intraparticle pores and adsorbed to walls of minute tortuous channels must desorb before diffusing into areas where microbial degradation is occurring.

130. Soil sorption of organic contaminants has been widely studied in recent years (DiToro, Jeris, and Ciarcia 1985; Hassett and Anderson 1982; Karickhoff 1981; Karickhoff, Brown, and Scott 1979; Landrum et a1. 1984; O'Connor and Connolly 1980; Voice, Rice, and Weber 1983). Brusseau and Rao (1989) reviewed the literature and concluded that diffusive mass-transfer resistances account for the most strongly sorbed contaminant fraction. This fraction is associated with intraparticle porosity, intra-aggregate porosity, and low-conductivity regions. Much of the soil sorption data has been limited to studies conducted with contaminant amended soils rather than anthropogenically contaminated soils from the field. Field contaminated soils, however, have been subjected to fluctuations in environmental factors such as temperature, moisture, leaching, sunlight, penetration by plant roots, and even perturbation by burrowing animals and insects. Contaminant binding in soils subjected to such dynamic changes may differ greatly from binding in amended soils. Time of contact between soil and contaminant also affects binding characteristics. Longer exposure times allow contaminants to migrate to intraparticle sorption sites in soils, clays, or organic carbon. As clay and organic carbon swell and shrink with wetting and drying cycles, adsorption to 
less accessible areas can increase. Desorption from these pores is much slower than from surface sorption sites. Therefore, laboratory results obtained by quickly amending soils with contaminants of interest must be verified using field contaminated soils. Information on desorption of contaminants of interest from field contaminated soils is extremely limited. Therefore, desorption studies using field contaminated soils will be necessary for most, if not all, of the contaminants of interest.

\section{Sorption Characteristics of Target Contaminants}

131. Soil adsorption of low molecular weight halocarbons, such as trichloroethylene (TCE), can occur from the vapor or liquid phase and generally follows a linear isotherm (Marrin and Thompson 1987). These compounds can adsorb onto several soil components, e.g., organic matter and clays. Generally, desorption may be relatively rapid, rendering the compounds readily bioavailable. However, evidence suggests that adsorption to certain expanding clays is irreversible, or that desorption is extremely slow.

132. Adsorption of BTEX compounds in organic soils is extensive and occurs at a rapid initial rate. However, slower adsorption also occurs, suggesting that significant periods of time may be required to achieve equilibrium of contaminant between soil solution and solids. Desorption of most of the contaminant into water occurs rapidly, but a smaller fraction of the contaminant desorbs slowly. Microbial degradation of toluene, for example, progresses at a rate directly related to desorption, i.e., biodegradation is rate limited by desorption (Robinson, Farmer, and Novak 1990). Implementation of pump-and-treat or in situ bioremediation requires knowledge of desorption rates and an understanding of the effects of sorption processes on microbial degradation.

133. Adsorption and desorption of aliphatic and aromatic hydrocarbons from the vapor phase is controlled by properties of the specific compound, soil properties, and, to a great extent, soil moisture content. As soil moisture increases, clays become hydrated, reducing available adsorption sites. Therefore, adsorption is controlled by organic carbon in saturated soils. Desorption rate is inversely related to soil organic carbon content and increases with soil moisture. Polyaromatic hydrocarbons, e.g., pyrene and phenanthrene, adsorb rapidly onto readily available surface sites in the soil and more slowly onto less accessible sorption sites (Karickhoff 1980). 
Desorption is a reversal of the adsorption process, except that the slowly adsorbed fraction is desorbed more slowly than it was adsorbed. Surfactants may enhance desorption of petroleum hydrocarbons.

134. Most of the data on MEK describes sorption to activated carbon used to treat wastewater streams. In these systems $0.094 \mathrm{~g}$ MEK are adsorbed per gram of carbon (Verschueren 1983). No information in the available literature was found on the adsorption/desorption characteristics of MEK with respect to soil systems.

135. Adsorption of TNT to soils exhibits a rapid initial component and a slower long-term component. Desorption is also rapid, but a small fraction is recalcitrant. Soil adsorption and desorption are governed by cation exchange capacity and clay content of soils (Pennington and Patrick 1990). TNT undergoes microbial degradation to several persistent intermediate compounds of greater environmental hazard than the parent compound (Kaplan and Kaplan 1982). Sorption properties and microbial availability of most of these intermediates have not been studied. A partitioning coefficient for RDX is similar to that of TNT, and clay content is more important than organic carbon content in RDX sorption (Sikka et al. 1980). Many of the important factors governing sorption of these two explosives are unknown. Therefore, special emphasis will be placed on sorption studies of TNT and RDX.

\section{Adsorption/Desorption Limitations}

136. Adsorption followed by a slow desorption rate often limits movement and bioavailability of contaminants. Therefore, special emphasis must be given to processes that control the rate and extent of adsorption/desorption in saturated and unsaturated systems. Partitioning of a contaminant between solid (sediment or soil) and aqueous phases (water) occurs via equilibrium or nonequilibrium processes at the solid/aqueous phase interface (Hill, Myers, and Brannon 1988; Brusseau and Rao 1989).. In equilibrium processes, the contaminant introduced on one side of the interface will move across the interface until a steady state exists (Thibodeaux 1979). If the rate of contaminant transfer across the solid/aqueous interface is rapid relative to convective and dispersive transport, equilibrium is established. In this case, the equilibrium distribution coefficients can be used to describe distribution of the contaminant between the two phases; the partitioning is equilibrium controlled, and the local equilibrium assumption is met (Valocchi 
1985; Parker and Valocchi 1986; Bahr and Rubin 1987; Valocchi 1988). The local equilibrium assumption means that the contaminant is in equilibrium with the solid and aqueous phases at any given point in the soil. This is true if the rate at which sorption reactions occur is fast relative to the velocity of pore water, i.e., sufficient contact time allows a local equilibrium distribution of the contaminant between aqueous and solid phases.

137. In nonequilibrium processes the distribution of contaminant between solid and aqueous phases has not reached equilibrium and, therefore, has a potential to change. The rate of change becomes important and may be controlled by such factors as external diffusion through a film, internal diffusion within the interstices of the solid phase, or reaction kinetics at the solid/aqueous interface (Weber 1972; Brusseau and Rao 1989). Generally, rate processes are controlled by mass transfer, rather than by the kinetics of chemical reactions. Mass transfer generally means movement of contaminant between phases, but movement may also occur between one or more regions within the solid/aqueous system. Transfer processes may, therefore, include (a) nonsteady diffusion through a stagnant layer of water immediately adjacent to the external surface of each solid particle, (b) intraparticle, aqueous-phase diffusion from desorption sites within the porous structure of the solid particle to the exterior, (c) intraparticle, solid-phase diffusion to desorption sites, and (d) combined internal (solid and aqueous phases) diffusion and external diffusion (film) (Weber 1972; Brusseau and Rao 1989).

138. Equilibrium processes are easily described mathematically, and chemical measurements for such processes are easier to quantify analytically than are those for nonequilibrium processes. Testing required to completely define all aspects of the nonequilibrium system is elaborate. Therefore, a general model can be used to account for all of the mass transfer processes that may be operative when one region cannot be assumed as rate controlling (Weber 1972; Thibodeaux 1979; Brusseau and Rao 1989).

139. Soils within aquifers are more or less constantly saturated with water, whereas vadose zone soils are only infrequently saturated. However, contaminant leaching from vadose zones is potentially greatest when the soil is saturated and water is moving through it. Therefore, when partitioning is considered, saturated conditions have relevancy to both aquifer and vadose zone soils.

140. The number of pore volumes of water that must be flushed through the soil profile to meet a cleanup goal during aquifer pump-and-treat 
operations depends on (a) the sorptive tendencies of the contaminant and the soil and (b) the pore water velocities attained during remediation (Mercer, Skipp, and Giffin 1990). Contaminant transfer from soil solids to the bulk flow of pore water may be controlled by either equilibrium or nonequilibrium processes, depending on hydraulic flux and sorption/dissolution kinetics. The local equilibrium assumption is often used to simplify the analysis of sorption effects on contaminant extraction by soil flushing. The local equilibrium assumption, valid when pore fluid velocities are low relative to the rate of contaminant desorption, is an important concept that significantly simplifies analysis of fluid extraction and in situ bioremediation technologies. Both equilibrium and nonequilibrium desorption introduces a tailing effect that requires longer pumping times and greater volumes pumped to reach a specified cleanup goal compared with a nonsorbing chemical. The amount of time and the volume of water needed to desorb contaminants can be significantly greater for nonequilibrium than for equilibrium-controlled processes.

\section{Effect of Sorption on Contaminant Biodegradation}

141. Organic compounds may be stabilized against rapid biodegradation through sorption to clay (Subba-Rao and Alexander 1982), other microorganisms (Herbes 1977; Bell and Tsezos 1987), and organic matter (Scott, Wolf, and Lavy 1982; Murphy, Zachara, and Smith 1990; Robinson, Farmer and Novak 1990). Sorption to clay can occur through electrostatic attractions or by interactions with coatings on particles. Using montmorillonite clay and benzylamine, Subba-Rao and Alexander (1982) found that increases in clay concentration decreased the percentage of aromatic compounds mineralized. Mineralization was more rapid than desorption during early periods of decomposition when amine level in solution was comparatively high. However, desorption was faster than decomposition during later periods when amine level in solution was very low. Orgam et al. (1985) showed that sorbed 2,4-D was completely protected from biological degradation, but that bacteria sorbed to soil surfaces degraded 2,4-D in the soil solution as efficiently as unattached bacteria. If a contaminant must be desorbed before degradation can occur, physical-chemical factors rather than microbiological factors may limit degradation.

142. The inability of microorganisms to degrade compounds sorbed to surfaces is attributed to the fact that sorption to surfaces renders some 
compounds inaccessible to enzymatic attack and/or incorporation into microbial cells. However, some compounds remain accessible to biological transformation even though tightly bound to sediments (Remberger, Allard, and Neilson 1986). Sorption may even increase the degradation of certain compounds, e.g., the triazine herbicides (Orgam et al. 1985; Armstrong and Chesters 1969; Russel1 et al. 1968), and compounds adsorbed to the surface of granular activated carbon (DeWaters and DiGiano 1990). Microbial access to contaminant sorbed to intraparticle spaces within the soil matrix is a potentially limiting factor for microbial degradation. Tortuosity of soil microchannels increases the difficulty for the organism and contaminant to interact by increasing the complexity of the path as well as the physical distance between microbe and substrate. Under these circumstances, diffusion limits degradation. Not only does pore size and tortuosity limit microbial access to contaminant, but microorganisms are capable of physically blocking pores, adversely affecting movement of fluids. This condition is well known as well-plugging and has even been demonstrated in rocks (Updegraff 1983). Methods of improving microbial access to contaminant in intraparticle spaces include use of electrolytic treatment, application of chemical measures that shrink the soil fabric, which effectively increases the pore size, and introduction of surfactants or taking advantage of surfactants produced by the microorganisms. Another potentially useful method of improving penetration of microorganisms into the soil matrix is introduction of ultrabacteria. Recent research has shown that many bacteria occur in the natural environment as small filterable particles induced by starvation. These organisms, the so-called ultrabacteria (Roszak and Colwell 1987), are sometimes less than $0.08 \mu \mathrm{m}$ in diameter. Lappin-Scott, Cusak, and Costerton (1988) were able to resuscitate ultrabacteria and grow them in sandstone cores, demonstrating their ability to move into small pores. Macleod, Lappin-Scott, and Costerton (1988) further demonstrated that the starved bacteria penetrated much deeper into sandstone core sections than their vegetative counterparts, but that the recovered cells reduced permeability because of cell growth and polymer production.

\section{Sorption of Microorganisms}

143. Organisms often live attached to surfaces, and surfaces tend to attract microorganisms from water because of net charge differences (Marshall 1980). Although sorption to soil surfaces enables microorganisms to scavenge 
nutrients and oxygen from passing water with little or no expenditure of energy, attachment precludes searching for substrates, such as polar organic contaminants which are likely to be adsorbed. Microorganisms may attach themselves directly to the surface of an insoluble contaminant that may or may not be subsequently utilized (Efroymson and Alexander 1991). Finally, microorganisms may produce substances that enhance the solubility of the substrate or influence its rate of movement into aqueous solution (Rosenberg et al. 1979; Zajick and Mahomedy 1984; Desai, Patel, and Desai 1988).

144. The attached biomass level in an aquifer is often much higher than the free-living biomass (Arvin et al. 1988). If flow of water and contaminant is limited because of low hydraulic conductivity in fine-grained soil, access of contaminant to the soil-attached bacteria will be reduced. In this instance, degradation would rely upon the free-1iving biomass. However, attachment does not necessarily reduce degradation (Orgam et al. 1985).

\section{Contaminant Desorption Enhancement}

145. Transport of organic contaminants can often be enhanced by introduction of surfactants and/or other materials to the system. Effects of these additives, termed facilitated transport, increase the apparent aqueous solubility of the contaminant and, thus, its mobility. Transport can be increased by cosolvents, organic colloids, and inorganic colloids (Nkedi-Kizza, Rao, and Hornsby 1987; Bengtsson, Enfield, and Lindqvist 1987; Gschwend and Reynolds 1987; Enfield and Bengtsson 1988; Huling 1989). Cosolvents are particularly effective in enhancing desorption of hydrophobic organic contaminants such as petroleum hydrocarbons or PAHs. When a mixed solvent system is present, e.g., water and methanol, acetone, or MEK, sorption does not follow the same partitioning behavior seen with water as the only solvent. Partitioning depends upon the relative proportions and characteristics of a mixture. Organic colloids, of which surfactants are one example, can serve as sorbent in sorption reactions with the contaminant or as solvent in solvent-cosolvent reactions. Surfactants form micelles, which can be readily transported in aqueous media. Inorganic colloids include clay, metal oxides, and submicrometer-sized precipitates of inorganic materials. Colloids that have adsorbed contaminants may pass readily through soil media. Failure to account for these systems can lead to greatly underestimating the potential mobility of contaminants. Furthermore, employment of facilitated transport can improve recoveries of 
contaminants from soils and, perhaps, increase bioavailability of contaminants for in situ bioremediation. 
146. The proper implementation of biotreatment technologies requires expertise in microbiology, chemistry, and engineering. Scientists and engineers have performed enough research to justify the belief that biotreatment technologies are technically feasible. The microbiologists have generally determined overall treatment conditions required to stimulate enough biological activity to mineralize many xenobiotic compounds; however, some research in the optimization of biological activity remains to be done.

147. Given input from the microbiologists on what conditions are required by the microbial communities to establish an effective treatment system, the engineer must determine ways of supplying the proper treatment environments conducive to these conditions. Unfortunately, especially with in situ applications, much of the research efforts in application of the technol. ogy remains to be done.

148. Various engineering aspects of several biotreatment systems, including the application scenarios used to date, promising application techniques, and problems associated with various implementation scenarios will be discussed in this part.

\section{Biological Treatment Technologies}

149. This section presents biotreatment technologies that can be implemented without excavation of soil, which are the technologies within the scope of this research program. There are other biotreatment technologies that do have great potential that will not be evaluated within this research program. These technologies require excavation of solls and are considered outside the scope of this program.

Saturated Soil Biotreatment Technologies

150. Implementation scenarios based on biological processes for remediation of saturated soil systems can be generalized into two main categories. These categories are based on the implementation methods used to apply the technologies. The first category is pump-and-treat (PAT) based 
biotreatment technologies. The difficulties remaining with this scenario are the effective extraction of the contaminants from the subsurface to the aboveground treatment systems. Once the contaminants are brought to the surface, then treatment is usually not difficult. A recent evaluation of PAT by Travis and Doty (1990) indicated that desorbing the contaminants from the soils to the extent that treatment levels are reached can be very difficult (Part $V$ presents discussion on the limitations associated with the desorption kinetics of the contaminants from the aquifer solids). The USEPA estimates that in many cases it can literally take tens of years to reach target cleanup levels (Travis and Doty 1990).

151. Once the contaminated groundwater is brought to the biotreatment system, treatment conditions required in terms of biological degradation are contaminant specific. Treatment of xenobiotic (man-made) compounds is well established in industrial wastewater literature. Much information, except for explosives, is available on the biological degradation of the target contaminants in this program. A review of the degradation potential of the target contaminants for this research program is presented in Part IV of this document.

152. The second category of biotreatment implementation scenarios for saturated soil systems is in situ treatment. With this scenario, the saturated zone or the aquifer essentially becomes the reactor. Various additives are injected into the treatment zone to enhance or establish biological activity. These zones of biological activity are referred to as biological active zones (BAZs). In situ treatment was first proposed in the 1970's by R.L. Raymond, who was granted a patent for his process for in situ bioremediation of petroleum hydrocarbons (Thomas and Ward 1989). Each implementation scenario will be discussed in more detail below.

\section{PAT based biological treatment systems}

153. PAT is an implementation scenario for treatment technologies; it is not a treatment technology within itself. PAT is always associated with some treatment technology. In reference to this program, PAT will be associated with biotreatment technologies. PAT was selected as an implementation approach in approximately 68 percent of the Records of Decision (RODs) listed for Superfund Sites (Travis and Doty 1990).

154. PAT can be associated with biological treatment processes as well as chemical/physical treatment processes. Typically PAT systems perform the following steps: 
Remove the contaminants from the aquifer

155. Contaminants are removed via flushing the contaminated aquifer using new groundwater entering the site or using treated groundwater recirculated back into the site from the treatment system (Keely 1989). The purpose of flushing is to remove the adsorbed contaminants from the contaminated aquifer solids. This process is usually sorption controlled and, depending on the contaminant, can be very slow (Keely 1989; Travis and Doty 1990).

Pumping contaminated ground-

water to the treatment system

156. Contaminated groundwater is collected and pumped to the surface using dewatering wells and/or trenches. This process can be facilitated using hydraulic or bentonite barriers to assist in the containment of the groundwater (Zappi, Shafer, and Adrian 1990). Improper design of dewatering systems can result in the treatment of large quantities of water that really do not require treatment. Excessive water collection can also dilute the influent to a bioreactor to levels that cannot sustain biological activity. The benefits of a good modeling support are realized during the design phase of the dewatering/recharge system. A good modeling effort will ensure that proper circulation of the groundwater through the contaminated areas will occur and that noncontaminated water will not be drawn into the collection system resulting in unnecessary treatment of larger volumes of influent.

Treatment of the contaminated groundwater

157. During the treatment phase, bioreactors or a physical/chemical treatment system can be used to decontaminate the groundwater. In the case of biological processes, proper selection of a biotreatment system is critical to the successful application of PAT systems. Limitations of the various bioreactors must be understood. Factors such as the minimal amount of substrate that will support an active biomass must be determined. Information on the minimal amount of contaminant that can support a microcosm is available from the microbiology community; however, a properly functioning biological system requires much more than simple cell maintenance. Proper operating bioreactors require proper settling biomass, biomass that does not slough because of the shearing forces of flowing influent, significant cell density to withstand shock loadings, and a generally healthy population that will ensure treatment goals are met. 
Injection of treated water (effluent)

158. Reinjection of the treated groundwater back into the aquifer is typically accomplished using recharge wells and/or trenches. The location of the injection points is dependent on whether the treated groundwater is recirculated back through the contaminated section of the aquifer or if the natural groundwater flow is used to flush the aquifer (i.e., no recirculation). Once again, proper modeling support is needed during the design phase to ensure efficient operation of the PAT system.

159. PAT can also be used as a means of preventing contamination from exiting a contaminated site and contaminating clean areas of the aquifer downgradient of the site. Contaminated groundwater is the mechanism for transport of the contaminants into the clean aquifer down-gradient of the site. PAT systems may be better used as a means of containment, not treatment, because of the slow desorption rate of some organic compounds. The boundary systems at Rocky Mountain Arsenal (RMA) are good examples of such systems (Zappi, Adrian, and Francingues 1990). RMA personnel operate two PAT systems, the North Boundary and Northwest Boundary treatment systems, which have average flow rates of $300 \mathrm{gpm}$ and $700 \mathrm{gpm}$, respectively. These systems use activated carbon to treat the collected contaminated groundwaters. The northnorthwesterly flow of the alluvial aquifer is halted by a hydraulic and soil/bentonite barrier system, the groundwater treated using the GAC, then reinjected on the clean side without disrupting flow.

160. Summary of problems associated with PAT systems. As discussed in Part $V$ of this document, the most significant limitation to the successful implementation of PAT is the transport of the contaminants from the contaminated zone to the bioreactor. The amount of contaminant that can be transported is either solubility or desorption limited. Most of the time, desorption of the contaminant from the aquifer solids into the groundwater is the limiting factor. Repeated permeation of a contaminated aquifer will eventually result in the decontamination of the contaminated solids in the aquifer. This process could take many years to accomplish; therefore, research into enhanced removal of adsorbed contaminant using such chemicals as surfactants could result in appreciable cost savings (enhanced removal is discussed in Part V).

161. As levels of contaminants in the aquifer are reduced, treatment system influent concentrations are also reduced. Variations in influent contaminant concentrations can be so significant that there is not enough 
contaminant (substrate) remaining to support biological growth, resulting in bioreactor failure. The effect of changing influent strengths needs to be investigated to determine minimum and maximum levels of the target contaminants that properly functioning bioreactors can withstand without failure. 162. All of the target compounds in this research program are biodegradable to some degree. Conditions conducive to biodegradation of the contaminants determine bioreactor design and configuration. These same conditions can result in significant losses of the contaminants because of abiotic pathways such as volatilization if conventional bioreactor types are used. For example, cometabolic degradation of TCE using methane can be theoretically under aerobic conditions. Supplying oxygen in a bioreactor can be very challenging without resulting in a significant loss of the TCE because of air stripping.

163. Another limitation of PAT is treatment or disposal of residuals. If activated carbon is used, then disposal or regeneration of spent activated carbon can significantly increase the operating cost of the system. Biological regeneration of spent activated carbon can potentially decrease the cost of handling spent carbon. The use of activated carbon will always have a place in site remediation activities. Some groundwaters are not contaminated at high enough levels of contamination to support an active bioreactor. Also, as contaminants are flushed from the aquifer, eventually influent quality will drop below the minimal amount of substrate required to support an active bioreactor.

164. Bioreactor types. Contaminants are removed from the aquifer using collected groundwater; therefore, PAT is most often associated with treatment of contaminated groundwaters because the groundwater is the transport mechanism for the contaminants. The type and design of the bioreactor employed is dependent on the contaminant and influent flow rate. Bioreactor systems are divided into two main reactor types: attached growth and suspended growth systems (Zappi, Teeter, and Francingues 1991). A brief description of each bioreactor type is presented below.

165. Attached growth bioreactors. Attached growth systems use bacteria that grow on fixed structures and remove their substrate as it flows by in the water circulated through the bioreactor. Attached growth or biofilm waste treatment processes, such as trickling filters or packed bed columns, were developed in the latter part of the 19th century (Peters and Alleman 1982). Over the years, many different types of attached growth processes have been 
successfully developed to treat domestic and industrial wastewaters (Antonic 1975; Li et al. 1986), landfill leachates (Opatken et al. 1988), and hazardous wastes (Donaldson et al. 1987).

166. There are many different types of attached growth bioreactors (Metcalf and Eddy, Inc. 1982). The more common include: trickling or rock filters, rotating biological contactors (RBCs), and submerged biological filters (SBFs). All of the above-mentioned bioreactors are aerobic; but anaerobic attached growth systems are also used, especially for high strength wastes. Typically SBFs are used as anaerobic attached growth bioreactors. The types of structural support materials used for attached growth can also vary. Typical support media include: rocks, wood slates or crates, plastic commercial packing media, such as Pall rings and Flexirings, GAC, and plastic corrugated sheets. Rittman (1982) evaluated the relative performances of several attached growth systems using a numerical modeling method. He concluded that a fluidized bed system was capable of producing a better quality effluent because of its operation in plug flow which evenly distributes the biofilm growth across the cross-sectional area of the bioreactor.

167. Advantages of attached growth systems over suspended growth systems are that attached growth systems are (Zappi, Teeter, and Francingues 1991; Banerji 1990):

a. Usually more resistive to shock loadings of highly contaminated influents or influents containing unusually high concentrations of toxins.

b. Usually require less operator attention.

c. Require lower influent concentrations to support biological activity.

d. Can be configured to eliminate or substantially reduce the amount of off-gasing of volatile contaminants from the bioreactor.

e. Washout of biomass at unusually high influent flow rates are minimized.

f. Detached or sloughed off biomass settle very well in clarification systems.

g. Require less energy per gallon treated.

168. Some disadvantages are:

a. That soluble cations such as iron and manganese can be oxidized in the bioreactor resulting in significant head loss across the system.

b. They typically produce a lower quality effluent than suspended growth systems. 
169. Suspended growth bioreactors. Suspended growth systems derive their name from the fact that the biological growth is held in suspension within the reactor. In a suspended growth bioreactor, high populations of bacteria are kept in suspension by mechanical means or, more commonly, by diffused air. The diffused air not only provides mixing, but also provides oxygen for the microorganisms. The bacterial activity, which results in the degradation of the contaminants, occurs in the aeration tank. As influent flows into the aeration tank, effluent flows into the clarifier. The clarifier separates the biomass from the effluent. Some of the thickened biomass is recycled back to the aeration tank to keep a constant population of microbes in the system. Some of the thickened biomass or sludge is wasted daily because the microbes are constantly multiplying.

170. The population of microorganisms in suspended growth systems is called activated sludge, and the biomass/effluent slurry in the aeration tank is referred to as mixed 1iquor. The microbial populations in the aeration tank are so great that they are typically measured using solids analyses such as total suspended solids or volatile suspended solids.

171. Although activated sludge may be the most popular suspended growth system, there are many other configurations that are feasible. Sequential batch reactors (SBRs) (Benefield and Randall 1980; Irvine et al. 1985) are similar to activated sludge, except that the aeration tank also serves as the clarifier. This system is operated in batch cycles. The cycles are fill, aerate, settle, and rest (idle). The SBR process can easily account for influent strength extremes and has great potential for application to contaminated sites. Other configurations of suspended growth systems are extended aeration (Gaudy and Lim 1980), contact stabilization (Gaudy and Lim 1980), and powdered activated carbon (PAC) activated sludge (Zimpro Inc., Meidel, and Peterson 1990).

172. Some advantages of suspended growth systems over attached growth systems are that suspended growth systems (Zappi, Teeter, and Francingues 1990; Banerji 1990):

a. Can usually handle higher daily organic loadings.

b. Will usually yield better quality effluents.

c. Are usually more dependable.

d. Are able to handle temperature extremes better.

173. In summary, suspended growth systems are either currently being used or are planned for use during the remediation of several of the sites 
reviewed. There are several reasons for this. One of the biggest reasons could be that much more engineering experience exists in the private sector for suspended growth systems than attached growth systems. Another reason is that more package suspended growth systems capable of handling low flows are commercially available than for attached growth systems. The third and final reason is that bench protocol for evaluating suspended growth systems are well documented (Metcalf and Eddy, Inc. 1982). Both systems have potential for treating contaminated groundwaters, but a thorough understanding of the limitations of both systems in terms of Army specific contaminants must be gained.

174. Treatment of xenobiotic compounds using aboveground bioreactors. The suitability of bioreactors for the degradation of a variety of complex organic contaminants has been demonstrated by many researchers (Kim and Maier 1986; Venkataramani and Ahlert 1984; Kelly 1987; Hamoda and Al-Haddad 1988). Most of the activities reviewed generally used the same technical approach to evaluate biological treatment technologies.

175. Sanford and Smallbeck (1987) used a mixed culture of bacteria and yeast to degrade a synthetic wastewater composed of $100 \mathrm{ppm}$ acetone, $50 \mathrm{ppm}$ MEK (2-butanone), and $125 \mathrm{ppm}$ methyl isobutyl ketone in bench-scale activated sludge bioreactors. They concluded that treatment of ketones was successful within $48 \mathrm{hr}$ of batch treatment utilizing a stable culture of microorganisms and yeast.

176. Palit and Qasim (1977) performed bench-scale treatability studies on leachates from a simulated landfill that contained various municipal wastes, including construction debris. The leachate chemical oxygen demand (COD) was diluted to yield an influent COD concentration of $366 \mathrm{mg} / \mathrm{l}$. A growth yield of 0.59 was estimated using graphical approximation methods. They concluded that contaminants typically found in municipal landfill leachates can be effectively degraded using activated sludge systems.

177. Kim and Maier (1986) evaluated the acclimation and biodegradation potential of chlorinated organic compounds in the presence of cometabolites. They were able to acclimate a culture of bacteria from a municipal activated sludge plant capable of degrading 2,4-D (2,4 dichlorophenoxyacetic acid) and 3,5-DCB (3,5 dichlorobenzoate) under aerobic conditions. Combined contaminant concentrations as high as $100 \mathrm{mg} / \ell$ were successfully degraded. They concluded that seed bacterial consortiums should contain as diverse a population of microorganisms as possible to increase the probability of plasmid exchange and 
that the acclimation phase should began with an influent containing very low concentrations of the target compounds to avoid inhibitory effects.

178. Bieszkiewiez and Pieniadz-Urbaniak (1984) evaluated the effects of benzene and xylene at concentrations as high as $75 \mathrm{mg} / \ell$ and $150 \mathrm{mg} / \ell$, respectively, on the work of an activated sludge system. They concluded that increased concentrations of the target compounds generally decreased COD removals, increased sludge volume index (SVI), increased the number of free swimming bacteria, and, finally, altered the morphology of the bacterial flocs.

179. Rozich and Gaudy (1985) evaluated the response of an activated sludge system to quantitative shock loadings of phenol. A phenol concentration of $500 \mathrm{mg} / \ell$ was evaluated as a base influent concentration. Initially, shock loadings of $1,000 \mathrm{mg} / \mathrm{l}$ of phenol were imposed on the activated sludge system without significant disturbances in treatment occurring. The activated sludge system was then shocked with $2,000 \mathrm{mg} / \ell$ of phenol, which resulted in a collapse of the stability of the system. They concluded that design engineers should design systems that will be treating possible inhibitory and/or toxic compounds activated sludge systems with high design sludge retention times (especially systems that could be subjected to periodic shock loadings of contaminants).

180. Hannah et al. (1986) evaluated the capability of different biological reactor types to remove various priority pollutants in the sub-ppm levels from wastewater influents. The bioreactor types evaluated were trickling filters, activated sludge, aerated lagoons, and facultative lagoons. They concluded that activated sludge followed by the facultative lagoon provided the best treatment as compared with the other bioreactor types. However, they did not indicate analyses of the off-gases from the bioreactors.

181. Zappi, Teeter, and Francinques (1990) evaluated the effectiveness of activated sludge and activated sludge with PAC addition for the removal of 2-butanone (MEK) from a contaminated groundwater from a Superfund Site. They concluded that biological processes were the main method of contaminant degradation and that off-gasing of the MEK only resulted in less than 5 percent of the total removal. They also concluded that the addition of PAC enhanced biological activity, resulting in increased gross pollutant removals.

182. Implementation of PAT systems. There have been many reported applications of PAT scenarios in the remediation of contaminated sites (McShane et al. 1986; Dietrich et al. 1988; Copa and Meidl 1986). Recent 
reviews of the success of PAT of meeting treatment objectives have not been favorable (Keely 1989; Travis and Doty 1990). Most of these applications have been for treatment of easy-to-degrade contaminants. Decision frameworks have been proposed to assist in the evaluation of the PAT scenario for site remediation (Mercer, Skipp, and Giffin 1990). A major knowledge gap associated with $P A T$ is the effect of varying influent concentration ranges on the performance of the bioreactors. Limited information exists on the proper design of the bioreactors for controlling such factors as contaminant loss and effective control of the oxidation of dissolved cations in aerated bioreactors.

183. Pretreatment of influents using chemical oxidation. The potential exists for the development of chemical oxidation as a pretreatment for conversion of refractory compounds to more biodegradable chemical intermediates. It is envisioned that such pretreatment technologies could convert biological system influents containing refractory compounds with relatively slow degradation rates (which increases the required hydraulic retention time) into influents requiring reduced treatment times. Significant reductions in the required treatment time can increase the technical feasibility as well as economics of blological processes for treatment of a contaminated groundwater.

184. Bowers, Gaddipati, and Eckenfelder (1989) used hydrogen peroxide catalyzed with a Fenton's reagent to increase the biological potential of several phenolic compounds. Their studies indicated that the intermediates produced via incomplete oxidation were an order of magnitude less toxic than the respective parent compounds.

185. Wang, Pai, and Latchaw (1989) evaluated reductions in inhibitory effects of o-cresol in anaerobic biological systems. They conclude that preozonation significantly improved the biodegradation potential of o-cresol. They further concluded that chemical intermediates from incomplete ozonation formed in the basic $\mathrm{pH}$ range were more biodegradable than those produced in the acidic $\mathrm{pH}$ ranges.

186. Miller et al. (1988) evaluated the effects of UV irradiation (photolysis) pretreatment for increasing the biodegradational potential of 2,4dichlorophenol and 2,4,5-trichlorophenol. They conclude that UV pretreatment greatly facilitates the removal of the two chlorophenols in activated sludge bioreactors through accelerated mineralization and binding of the polar bonds.

187. In summary, improvements in the biological treatment potential using chemical pretreatment technologies can result in an improvement in 
treatment efficiency and costs. Recent studies indicate that pretreatment with chemical oxidation processes with and without chemical and photolytic catalysts can significantly improve the effectiveness of biological processes. In situ biological technologies

188. This form of biotreatment has seen very limited use in terms of full-scale remediation of contaminated aquifer systems. Many vendors in the private sector have claimed success; however, there is very little data with which to verify their claims. The vendors claim proprietary concerns or client protection for not verifying their claims of success.

189. The concept of in situ biotreatment is simple. The actual implementation can be very difficult. In most cases, the basic microbiological conditions required for in situ treatment are relatively well understood. The major knowledge gaps are the applied aspects of microbiological concepts and the proper design of an implementation scenario.

190. The major components required for successful implementation of in situ biotreatment are listed and discussed below.

Microbial populations

191. The presence of active microbes in an aquifer being remediated using an in situ biological technology is paramount. Most studies have indicated that aquifer systems have the appropriate microbial types required for contaminant degradation. This is especially true for sites that have been contaminated for several years allowing native microbial strains to become acclimated to the contaminants. If the proper microbes are not present, then an exotic microbial inoculum or seed can be added (there is little documentation that this will be successful with in situ applications). In the case of chemical spills, appropriate microbes may not be present. Therefore, standardized protocols for evaluating existing microbial ecosystems in soils must be developed. Moris and Novak (1989) examined the mineralization of tertiary butyl alcohol (TBA) using nitrate as an electron acceptor in two previously uncontaminated soils. They concluded that one soil had appropriate microbes (denitrifying bacteria) to mineralize TBA while the other soil did not. They further concluded that understanding the ecological system of a soil during the remediation process is critical to successful technology implementation.

192. Since the "appropriate" microbes are usually already present within the aquifer, one must question why the contaminant(s) still persist. The reason why the contaminants still persist is that the soil ecosystem usually has a deficit of a single or many key constituents preventing an increase 
in biological activity. Once the key component(s) are added, BAZs can be established. Proper understanding of the microbial ecosystem is very important in selecting the additives required to efficiently and effectively degrade the contaminants. These concepts are addressed in much more detail in Part IV of this document.

Electron acceptors

193. Part of the microbial degradation process of contaminants includes the transfer of electrons or energy via an electron acceptor (EA) (See Part IV). These EAs are potentially one of the key constituents missing from potential BAZs. Typical EAs are oxygen (aerobic), nitrate (anaerobic), manganese and iron (anaerobic), and carbon dioxide (anaerobic). Using soil columns, Arthur et al. (1988) concluded that oxygen (or some EA) was the limiting factor in establishing an active microbial community for their systems. They further state that their conclusion substantiates the findings of others (American Petroleum Institute 1978). The mechanisms of EA usage by various microbes is fairly well understood. The main problem is how to get the EAs to the microbes at significant concentrations. Delivery of EAs presents several problems in terms of additive losses (sinks) during transport through porous media. Raymond, Jamison, and Hudson (1976) reported significant problems with both the transport of oxygen and nutrients into a contaminated aquifer.

194. Aerobic degradation of most of the target compounds in this study offers the fastest degradation rates; however, oxygen transport through aquifers is very difficult (Raymond, Jamison, and Hudson 1976; Thomas and Ward 1989). The two main mechanisms for getting oxygen to BAZs are oxygen via diffused air (Raymond, Jamison, and Hudson 1976) and hydrogen peroxide transported through the aquifer (Thomas and Ward 1989).

195. Hydrogen peroxide is very reactive. Its transport through an aquifer can be very difficult (Thomas and Ward 1989). Natural soil constituents such as iron can serve as a Fenton's reagent, which catalyzes the rapid degradation of hydrogen peroxide. Also, the presence of organic matter can result in the reaction and degradation of hydrogen peroxide (Thomas and Ward 1989). Soil constituents and threshold problem concentrations that initiate hydrogen peroxide degradation, posing problems with transport through aquifer systems, must be identified. Once this information is developed, design criteria and guidelines can be established. This information will be very useful to the design engineer during feasibility evaluation of in situ bioremediation. 
196. Wilson, Smith, and Rees (1986) evaluated the use of nitrate as an alternate electron acceptor (AEA) to degrade organic contaminants (BTEX).

They concluded that these compounds could be successfully degraded using AEAs. Riss and Schwiesfurth (1988) using soil slurry systems operated in a batch mode successfully used nitrates as an EA in the degradation of fuel oils. Although anaerobic degradation is usually kinetically slower, the sources of these AEAs seem to be more stable in an aquifer environment. Increased stability results in better transport properties in porous systems. Therefore, emphasis will be placed in this program on evaluating AEAs for establishing BAZs .

197. One innovative technology that has potential for providing oxygen to BAZs is colloidal gas amphrons (CGAs). CGAs were first produced by Sebba (1971). Since then they have been used in a variety of applications. They have been used in air flotation systems (Gregory, Barnett, and Deluise 1980; Ciriello, Barnett, and Deluise 1980) and to remove finely dispersed oil droplets (Sebba and Bennett 1981). Two methods are used to produce CGAs (Sebba 1971; Sebba and Bennett 1981). One method involves the passing of a dilute surfactant through a venturi throat at high rates. The second and newer method uses a spinning disk immersed in a surfactant solution. Michelsen, Smith, and Suggs (1988) have developed a method for producing CGAs or microbubbles at the rate of 30 to $70 \mathrm{l} / \mathrm{min}$ with low energy requirements. The current minimum size is greater than $15 \mu \mathrm{m}$. Michelsen, Smith, and Suggs (1988) describe some of the physical properties of CGAs. Recent studies by Michelsen, Smith, and Suggs (1988) indicate promise for using CGAs as a source of oxygen in water because of their fluid-like properties. More study in the area of microbe usage of the CGAs, the oxygen demand exerted by the surfactants used to manufacture the CGAs, and improved production methods must be performed before this technology can be applied. It is also possible to charge CGA's with microbial inocula and nutrients, thereby, introducing a true seed for the stimulation of BAZ production. Combining this technology with electrokinetics, which will be discussed later, may offer an innovative method of transporting the CGAs through tight aquifer systems.

198. One final consideration is that the participants of this research program must be sensitive to regulatory considerations that may eliminate technically preferred AEAs. A good example of this is nitrates that have a drinking water standard of $10 \mathrm{mg} / \ell$. It is difficult to believe that some states will allow the introduction of nitrates into an aquifer. Therefore, an 
evaluation of regulations that may prevent application of in situ technologies must be made. These restrictions will not hinder research in this program. However, it is believed that to be prudent, one must be sensitive to these issues.

Nutrients

199. Another key constituent that potentially could limit the extent of $\mathrm{BAZ}$ coverage is nutrients. Microbes require a variety of primary and secondary nutrients. The two primary nutrients required are nitrogen and phosphorous. Swindoll, Aelion, and Pfaender (1988) evaluated the effect of nitrogen and phosphate addition on the biological degradation rate of various aromatic compounds in subsurface soil samples. They conclude that the addition of these nutrients generally induced microbial mineralization of the contaminants indicating that the systems were nutrient limited.

200. Adsorption of the nutrient species can make transport of these species difficult. For example, ammonia, which is a popularly selected source for nitrogen, is actually used as one of the standardized methods to determine the cation exchange capacity of soils (USEPA 1986). Cation exchange capacity is the measurement of the soils ability to retain cations within the pore spaces and clay layers of the soil matrix.

201. A general rule of thumb typically used in wastewater treatment for the amount of nitrogen and phosphorous required is expressed in the form of the $C: N: P$ ratio. This ratio is the amount of substrate to nitrogen nutrient to phosphorous nutrient (Metcalf and Eddy, Inc. 1982) required by a microbial community. Typical C:N:P ratios range from 100:20:1 to 100:50:10. Recent communications with research personnel at Cornell University indicate that $C: N: P$ ratio has a much more significant effect on the mineralization rates of xenobiotic compounds than traditionally thought (personal communication with Dr. Martin Alexander 1990). Alexander's findings indicate that an increased presence of $\mathrm{N}$ and $\mathrm{P}$ as compared with those suggested by traditional $\mathrm{C}: \mathrm{N}: \mathrm{P}$ ratios can significantly increase the degradation rate.

202. Other inorganic nutrients are also required by microbes. These other nutrients are commonly referred to as secondary or micronutrients. They represent a host of inorganic compounds, but are rarely considered limiting because they are typically at appreciable amounts in soil systems. 203. Much 1ike EAs, the biggest problem with nutrients and in situ biotreatment is transport of the nutrients to the BAZs. Candidate nutrient complexes must not adsorb or react with constituents in the soil matrix thus 
preventing their introduction to the BAZs located far away from the injection points. Van der Zee, van Riemsdijk, and de Haan (1985) evaluated the fate of phosphate in noncalcareous soil. They conclude that reaction of phosphate with inorganic complexes can significantly reduce phosphate mobility. Nutrients can precipitate in an aquifer resulting in a reduction of the aquifer hydraulic conductivity (Thomas and Ward 1989). Reduction in the hydraulic conductivity of an aquifer being remediated can be detrimental to the success of remediation activities. Groundwater flow through a contaminated area is the only means, outside of excavation, by which the aquifer can be remediated. Therefore, extreme care must be taken when injecting additives so that the aquifer is not essentially "sealed" because of severe reductions in hydraulic conductivity.

Introduction of additives

204. Based on the above discussions, it is easy to see why injection systems are so important to an in situ biotreatment system. An injection system is only as good as the groundwater hydraulic model used to design and determine proper location. Injection systems also represent a large portion of the costs associated with in situ technologies. Wells are expensive to install, and pumps are expensive to operate. Injection of additives must be carefully planned. Nutrients and EAs should not be made simultaneously to prevent excessive growth of microbes around the injection points resulting in localized clogging of the aquifer (Thomas and Ward 1989).

205. One promising and innovative technology that has not been evaluated as a means of transporting additives is electrokinetics (EK). EK was developed by Casagrande in 1947 to dewater and, thereby, stabilize railroad foundations in Norway (Acar, Hamed, and Gale 1990). EK has the potential to electronically move water through low permeability media at velocities much higher than those obtainable even using high hydraulic gradients. It is envisioned that by properly locating EK electrodes, additives can be introduced into tight formations of an aquifer system that would have been dead zones in the aquifer during site remediation. These dead zones could contain contaminants within the pore spaces that could serve as a continual source of contamination throughout the remediation of the site. Dead zone contaminant sources are one of the contributing factors to the failures of PAT and in situ technologies. Acar, Hamed, and Gale (1990) present a good description of EK and the state of the art in current applications. 
206. EK also has the potential to move inorganic and organic compounds through water systems at rates much faster than the respective velocity of the pore water. If additives or possibly microbes (realizing that the effect of EK-induced electrical fields on the microbes must be studied) could be transported through aquifer systems with little water displacement, then the cost of remediating contaminated sites in terms of time and pumping costs could be substantially reduced. Also as mentioned above, EK could be used to establish BAZs in dead zones that could not have been established using conventional injection systems.

Groundwater modeling

207. The selection and design of remedial actions has been largely accomplished through the collection of field data and best engineering judgment. These approaches could become very costly in areas where the site characteristics differ substantially from previous known applications. Predictive tools could be used to make quantitative evaluations of remedial action performance in complex environments. Application of best engineering judgment in association with the quantitative results would then lead to more accurate and confident decisions.

208. Models are used to support the analysis of alternatives and aid in the conceptual design of the selected alternative. Some examples include the location of wells, the number of wells, and pumping rates required in the capture and treatment of a contaminated plume. Another example is the injection of chemicals to destroy or immobilize a pollutant. A contaminant transport model can be used to evaluate the size of the treatment zone and the extent to which chemical injection will reduce the groundwater contamination. 209. The use of models in the bioremediation program will aid in the selection of cost-effective alternatives and in the actual operation of the selected alternative. Chemodynamic pathways evaluated in the laboratory will be incorporated in the models to project field performance. Areas where the estimation tools can greatly assist site remediation planners are:

a. Location (arrangement) of pumping wells and rates.

b. The movement of nutrients in the subsurface environment.

c. The movement of oxygen (peroxide) in the subsurface environment.

d. Simulation of biogeochemical processes as described by the laboratory investigation.

e. Simulation of contaminant exposure levels away from the cleanup site. 
Application of in situ biotreatment

210. A review of selected case studies on site remediation activities using some form of in situ biological treatment of contaminated aquifer systems indicated that most sites were relatively small in terms of area (typically a few acres) and usually contaminated with petroleum hydrocarbons. However, some case studies reviewed were contaminated with chemicals other than hydrocarbons, such as ketones and solvents. Most of the information obtained was from either survey (summary-type) papers authored by various researchers or papers with very limited detail from private engineering firms (private sector). In general, these sources of information lacked much detail in terms of implementation techniques employed. There was also a lack of confidence felt concerning the comprehensiveness of the evaluation methods used to monitor remediation progress during the majority of the case studies reviewed.

211. Al1 of the case studies reviewed except for the two Air Force case studies generally indicated successful treatment. This is not surprising since most pilot- or full-scale efforts have been performed by the private sector. During the course of this evaluation, it was noted that the private sector seemed to be hesitant in sharing information on their past efforts in biotreatment. If they had a successful bioremediation project, they did not want to share their techniques. If they experienced an unsuccessful attempt at bioremediation, they were even more hesitant to share this information. Neither case is surprising since it must be kept in mind that the private sector is situated in a very competitive world.

212. A review of 26 case studies is presented in Appendix A of this document. A summary of the key components of the various site implementation scenarios discussed in Appendix $A$ are presented in the following paragraphs of this section. Efforts are ongoing for retrieval of more case studies. There is much information that can be gained from successes and, even more important, failures experienced during bioremediation activities. Various oxygen sources were used during the remediation efforts reviewed. Eight sites used air sparging as a means of introducing oxygen into the BAZs, while eight other sites used hydrogen peroxide, typically at 35 percent concentration, as an oxygen source. Two sites used a combination of air sparging and hydrogen peroxide injection to provide required levels of oxygen to the BAZs. In both cases where a combination of air sparging and hydrogen peroxide were used, air sparging alone was initially attempted with limited success. Later, hydrogen 
peroxide addition was initiated as an attempt to improve oxygen transport into the BAZs. One site used a combination of nitrate and sparged air as a source of electron acceptors.

213. Eighteen. sites used native (indigenous) bacteria for establishment of BAZs. Five sites used commercial inocula for establishing BAZs. This trend generally follows the experiences shared by various people interviewed who are actively involved in site remediation activities using biological processes. Most efforts indicated the presence of contaminant degrading microorganisms at the sites. The native bacteria seemed to be nutrient and/or EA limited because BAZs were established once these additives were provided.

214. The geologic formations of all of the sites were composed of predominately high permeability sands. Most sites were relatively shallow, completely saturated aquifers. These conditions seem optimal for attempting in situ biotreatment.

215. In summary, the pulse of the environmental remediation industry is that private sector is generally overly optimistic with in situ biotreatment as a developed technology, while the research community is much more pessimistic. Based on review of the available literature, it is felt that in situ biotreatment is technically feasible with many implementation questions yet to be answered. It is not a technology that is readily implementable unless suitable site conditions exist. Ideal site conditions include highly biodegradable contaminants contaminating a shallow, highly permeable, welldefined aquifer. Two problems that were identified by the private sector during the review of the various case studies were reduction in aquifer permeability and inability to introduce appropriate amounts of EAs into the BAZs. As concluded earlier, the biggest factor limiting implementation of in situ biotreatment seems to be the inability to properly introduce additives into contaminated aquifers.

\section{Summary of findings}

216. There is not a clear-cut optimal system in terms of whether to treat a site using PAT or in situ biotreatment methods. Several factors must be incorporated into the decision framework. Much of the information required is not clear in terms of how key problems associated with each scenario can be addressed. These knowledge gaps will be addressed as part of this research program.

217. Even after some of the knowledge gaps have been bridged, the limitations of both scenarios must be understood (this is true for all treatment 
technologies). The more understanding of all potential technologies that is gained, then the more technically and economically sound decisions will be made in terms of an optimal selection of a treatment technology.

218. Other considerations must be kept in mind when choosing whether PAT or in situ should be attempted. Time limitations often are more pertinent in the decision framework then technical feasibility. This program will hopefully give the design engineer enough information on the technical and regulatory issues that currently hinder application of the technology.

219. This program will also lay down the framework for combining PAT with in situ treatment by an increased understanding of the two technologies. A potential situation where implementation of the two would be when a PAT system has an influent of reduced strength such that a bioreactor cannot be operated any longer. At this point, in situ treatment can be used to reduce the residual amount of contamination in the aquifer to acceptable levels.

220. In closing this section, biotreatment offers a potentially great alternative to physical/chemical treatment technologies. A better understanding on how to implement this technology will be gained through this research program.

Unsaturated Soil Biotreatment

221: The concept of biological treatment of the unsaturated zone or vadose zone is relatively new. There has been little or no real field data on the successful implementation of biological technologies for remediating vadose zones (NOTE: land-farming is not considered an in situ technology with respect to this program). The intent of this research program is to evaluate various potential implementation scenarios that may result in biological treatment of vadose zones or joint treatment using in situ volatilization and biological treatment.

222. Some proposed implementation scenarios for contaminated vadose zones are presented below:

Flooding the site

223. This scenario basically converts a vadose zone into a saturated soil system by raising the water table of the site into the contaminated zone. Once saturated, the site remediation can be handled much like other saturated soil systems. It is obvious that this scenario must be limited to medium to small sites because of the restrictions associated with flooding of a site. 
It is difficult to use aerobic processes to remediate flooded sites because supplying oxygen to the BAzs can be very difficult but not impossible.

Anaerobic biological degradation approaches seem to offer an easier means of remediating vadose zones using flooding techniques.

Induced aeration

224. This method of vadose zone remediation uses in situ volatilization technologies such as soil venting to supply oxygen to the BAZs. Arthur et al. (1988) conclude that forced venting of air or induced aeration can result in a significant increase in the biological activity of a soil. Their studies include the degradation of JP-4 in unsaturated soil columns.

225. These sites are saturated because saturating would severely hinder gas transport through the pore spaces. However, microbes require moist or saturated environments. Therefore, induced air treatment scenarios must supp1y water to keep the BAZs moist. Water used to maintain moist conditions can be charged with nutrients. This offers a mechanism for keeping the required amount of nutrients available to the microbes in the BAZs. Little is known on the exact moisture levels required to optimize biological activity. Too much moisture can hinder transport of oxygen through the soil, while too little moisture can hinder biological growth.

\section{Activated Carbon Regeneration}

226. The use of GAC beds for adsorbing dissolved organic matter from wastes promotes biological activity in the system. This biological activity often gives higher organic removals than predicted by adsorption alone. In addition, the biological growth can foul the GAC beds, which may cause higher hydraulic head loss (plugging) and anoxic conditions (H2S odors). The use of fluidized carbon bed can eliminate some of the plugging and anoxic condition problems if the fluidizing liquid has sufficient oxygen.

227. The concept of using microbes to degrade the adsorbed organic compounds on the activated carbon particles is called "bioregeneration." The enhanced organic removals by biologically active carbon systems has been attributed to "bioregeneration." In addition, in many GAC columns the life of the media (before regeneration) has been extended beyond the theoretical values because of the biological activity in the column and indirect "bioregeneration" (Benedek 1980). 
Past efforts in the

bioregeneration of spent GAC

228. Although activated carbon has been used for drinking water treatment for quite some time, the biological activity associated with the activated carbon was-not noticed for quite some time, because the organics removed were generally biostable natural compounds that did not cause significant biological growths. It was not until activated carbon treatment was proposed for wastewater that investigators observed biological growth and activity (Benedek 1980).

229. Some of the advantages claimed for combined adsorption and biological degradation are (Kim and Pirbazari 1989):

a. Enhanced biodegradation of slowly biodegradable compounds by their retention on carbon surfaces.

b. Favorable environmental conditions for microbial growth through enrichment of substrate and oxygen.

c. Protection of microorganisms from toxic substances by carbon adsorption.

d. Elimination or dampening the effect of concentration fluctuations of the adsorbable compounds in the feed.

e. Lower capital and operational costs for a single reactor.

$\underline{f}$. Lower energy expenditure in comparison with thermal or steam regeneration of the carbon.

230. Much of the reported literature dealt with modeling the performance of GAC systems that are biologically active. Benedek (1980) proposed a uniform biofilm model for the biologically active carbon granule, where the mechanism for the organic removal was based on the following steps:

a. Transfer from solution to the bacterial film.

b. Simultaneous diffusion and reaction in the film.

c. Adsorption of nondegradable and nondegraded organics.

d. Desorption of organics from the carbon when feed concentration decreases below equilibrium concentration or by bacterial acclimation to previously poorly degraded organics.

e. Biodegradation of the desorbed organic molecules.

231. The details of the mathematical model and its solution are given by Peel and Benedek (1976). The model when applied to pilot plant data was reasonably successful in predicting the performance.

232. Speitel et al. (1990) proposed a more complicated model that included consideration of processes occurring in the liquid film surrounding the GAC particles in the biofilm attached to the GAC and within the GAC. They 
used radio-labeled phenol and para-nitrophenol (PNP)adsorption/desorption/ biodegradation in laboratory GAC columns to validate their model. The concentrations of phenol and PNP used in the experiments were 1 ess than $100 \mathrm{mg} / \ell$, simulating levels encountered in water treatment plants. The model was able to predict the bioregeneration rate of the carbon after adjustment of some of the coefficients, but it failed to predict the liquid phase substrate concentration.

233. In a related study, Speitel et al. (1990) reported that sensitivity analyses identified three important parameters in their earlier models that affected the predictions. The parameters were: the Monod half-saturation coefficient, the GAC surface diffusion coefficient, and the initial biomass content. Smaller values of the Monod coefficient than experimentally measured were needed to fit experimental data satisfactorily. The GAC surface diffusion coefficient mainly affected the sorbed substrate biodegradation rate. Larger values reflected more rapid transport of the substrate, which promoted an increased bioregeneration rate. Some evidence of irreversible adsorption was noted based on the fact that predicted bioregeneration rates exceeded the measured rates during the later portions of column operation. The amount of biomass initially attached to the GAC caused an effect on the biodegradation of substrate from both the sorbed and liquid phases. The amount of initial biomass was difficult to determine experimentally. This was probably the largest impediment to developing a good predictive model.

234. Kim and Pirbazari (1989) developed a model for a biologically active granular activated carbon in a recycle fluidized bed configuration. This type of reactor configuration had less problems with clogging of the bed and provided better mixing. The model incorporated liquid film transfer, biodegradation and diffusion in biofilm, adsorption onto activated carbon, and biofilm growth. Computer simulations of the model were used for adsorber performance predictions from parameters obtained from adsorption equilibrium and kinetic studies, biokinetic experiments, and correlation techniques. Two easily biodegradable substrates, glucose and sucrose, and two actual wastewaters, dairy waste and a landfill leachate, were used to compare the predicted model profiles with experimental data for abiotic and bioactive adsorbers. The predicted profiles matched the experimental data fairly well. 235. Tesezo and Benedek (1980) investigated the removal behavior of nondegradable organic compounds generated during the biological treatment process by various adsorbents to see if these compounds could be removed by 
biologically active adsorbent columns. They found that granular activated carbon was capable of adsorbing the biogenerated residual organics. They observed no biogenerated residual organics in the effluent of the fluidizedbed reactor to which phenol was being fed at 15 to $50 \mathrm{mg} / \ell$. The fluidized-bed reactor with granular carbon had been seeded with acclimated microbial cultures. Presumably, the biogenerated residual organics were removed by adsorption on the carbon. The phenol removal by the reactor was high initially because of adsorption on the carbon. The removals then decreased as the capacity of carbon to adsorb the phenol got exhausted. With time, the removals slowly increased to high levels because of biodegradation by the attached microbes. The biodegradation rates of phenol were high as long as the dissolved oxygen levels were maintained high. Anoxic conditions caused phenol biodegradation rate to be more than order of magnitude slower than the rate under aerobic conditions.

236. Schultz and Keinath (1984) investigated the mechanisms that are involved in the removal of phenol in the powdered activated carbon treatment (PACT) system. They found that the respiration rates from acclimated PACT and suspended biomass cultures were about the same, which refutes the hypothesis that PAC addition enhances the biodegradation rate of phenol. Complete bioregeneration of carbon did occur when phenol was used as a substrate. The controlling mechanism for bioregeneration is desorption by a lowering of the liquid-phase phenol concentration and subsequent biodegradation. The metabolic end product organics of aerobic metabolism were adsorbed irreversibly to the carbon and could not be significantly desorbed by dilution or displaced by phenol. The adsorption of the metabolic end products accounts for the increased removals observed in PACT systems. The irreversible adsorption of metabolic end products explains the incomplete bioregeneration observed previously by others and supports the hypothesis that reversibility of adsorption is the controlling mechanism for bioregeneration. A very small fraction ( 4 percent) of the metabolic end products adsorbed on the carbon could be biodegraded by direct bacterial or exoenzymatic action.

237. Kim et al. (1986) studied the adsorption and biodegradation of phenol (up to $2000 \mathrm{mg} / \mathrm{l}$ ) in an anaerobic fluidized GAC reactor. They observed bioregeneration of the carbon media through desorption of adsorbed phenol and subsequent biogas production. They found that the contribution of biogas production, adsorption, and biomass production were all important in the removal of phenol. 
238. Speitel et al. (1989) evaluated the biodegradation of synthetic organic chemicals in GAC columns. Such biodegradation could extend the GAC column service life by in situ bioregeneration and also provide a more stable operation of the columns. They studied the biodegradation of p-nitrophenol (PNP) , 2,4-dichlorophenol (DCP), and pentachlorophenol (PCP) over a concentration range of 1 to $25 \mathrm{mg} / \ell$. Biodegradation measurements were made of both sorbed and liquid-phase substrate. PNP and DCP were found to be readily biodegradable in the GAC columns, but PCP degraded at a much slower rate. Significant biodegradation of sorbed PNP occurred only. The DCP appeared to be irreversibly sorbed onto the carbon, with negligible bioregeneration occurring even though the liquid phase concentration was lower than equilibrium levels. The absence of bioregeneration with PCP was due to its slow biodegradation rate.

239. Dobrevski and Zvezdova (1989) investigated the effect of activated carbon pore structure on the bioregeneration process. They used a phenolictype waste at an initial concentration of $400 \mathrm{mg} / \ell$ in a completely mixed batch reactor. Heterogenous microorganisms and cell extracts were added to the reactor for bioregeneration experiments. They found that activated carbon media with predominant pore size between 5 and $50 \mathrm{~nm}$ were more suitable for bioregeneration than other carbons examined. The cell extracts were able to bioregenerate a higher capacity of the carbon $(\mathrm{mg} / \mathrm{g})$ compared with microbial cells. As much as 67 percent of the original carbon adsorption capacity was restored by cell extract bioregeneration.

240. Off-line bioregeneration of GAC was studied by Goeddertz et al. (1988). They derived a mathematical model for the off-line bioregeneration reactor, which consisted of a recirculating expanded bed reactor, and compared the predicted bioregeneration of the carbon with the measured values. The reactor was supplied with acclimated bacteria, nutrients, and dissolved oxygen. Phenol was used as a substrate for the experiments. The duration of bioregeneration ( 1 to 4 days) and initial reactor volatile solids (biomass) (100 to $1,000 \mathrm{mg} / \mathrm{l}$ ) were the two parameters that were varied during the experiments. As expected, increased biomass and longest bioregeneration period gave the highest percent regeneration. The maximum bioregeneration approached 80 percent of the original capacity of the carbon. It was theorized that 20 to 25 percent of the phenol was irreversibly adsorbed. The upper limit of regeneration would depend upon the adsorption characteristics of the carbon 
and the limiting phenol concentration in solution. The mathematical model proposed provided a good representation of the process.

\section{Summary of findings}

241. Most of the work reported deals with compounds that are quite biodegradable and also adsorb/desorb easily, e.g., phenol. Even with phenol there was sometimes incomplete bioregeneration, although some researchers found complete bioregeneration of phenol-saturated PAC.

242. The microbial metabolic end products adsorb well on the carbon surface, although they do not compete for the same sites as the phenol. A large fraction of the metabolic end products are irreversibly adsorbed onto the carbon and are not easily biodegraded while in this state. This would cause an incomplete bioregeneration of the activated carbon.

243. Very little work has been done with hazardous compounds at higher levels (10 to $100 \mathrm{mg} / \ell$ ). Speitel et al. (1989) evaluated the biodegradation of substituted phenols in GAC columns at 1 to $25 \mathrm{mg} / \ell$ concentration range, a range common in water supply applications but not in situations where hazardous wastes are encountered. Kim and Pirbazari (1989) did use a landfill leachate containing milligrams-per-liter levels of some hazardous wastes, but the leachate had large amounts of biodegradable organics (BOD5 $-1,603 \mathrm{mg} / \ell$ ), which made the results rather unique.

244. The mechanism for GAC bioregeneration proposed by most investigators is as follows: substrate adsorption onto the carbon (fairly rapid process); biofilm growth and biodegradation of the substrate, if external substrate level decreases below equilibrium levels; desorption of substrate from the carbon occurs, which is subsequently also biodegraded; and, finally, some fraction of the substrate (or a metabolic product) could be irreversibly adsorbed that could not be bioregenerated. For some compounds, the desorption would govern the bioregeneration rate; and, for others, slow biodegradation rate of the compound would be the rate-limiting step.

245. Most of the studies reported used fluidized bed granular activated carbon reactors for experimentation on bioregeneration under aerobic conditions, although one report mentioned the use of anaerobic fixed bed and fluidized bed GAC reactor (Kim et al. 1986).

246. In summary, technically, bioregeneration of spent activated carbon seems technically feasible. Most of the research efforts reported bioregeneration of spent GAC as a secondary objective. Also, the majority of these studies used phenol as the substrate. Research using target compounds for 
this research program must be performed as well as evaluating improved methods to increase the biological degradation kinetics of spent GAC bioregeneration.

Contaminated Gas Biotreatment

247. This section describes some of the work that has been performed on biological treatment of contaminated gases. Also presented is a brief overview of some of the physical/chemical (abiotic) processes currently being used to treat these gases.

Abiotic processes for treatment of contaminated gases

248. Blaney and Branscome (1988) reviewed existing technologies for decontamination of off-gases from air stripping units. Three major types of technology groups were identified. These groups were activated carbon, thermal treatment, and catalytic incineration. It was concluded that activated carbon was by far the most commonly used treatment. No clear costs were given for carbon usage. Typically, the off-gases from air stripping units are heated prior to entering the GAC beds to lower the humidity, because vapor phase GAC adsorption works best at humidities of less than 50 percent. Another case study presented by Blaney that used a secondary combustion unit operated at $1,800^{\circ} \mathrm{F}$. This form of off-gas treatment is expensive and has a bad public perception associated with it.

Potential of biological processes for treating contaminated gases

249. McFarlane et al. (1981) evaluated the fate of benzene in soil, plant, and water systems. They concluded that microbial activity resulted in the complete mineralization of benzene to predominately carbon dioxide. Although this study was directed more toward the atmospheric fate of benzene in terms of mineralization by soil and plant microbial populations, the study does indicate that gas phase organic compounds are mineralized by solid phase based microbes.

250. Kosky and Neff (1990) summarize the state of the art of gas phase for biotreatment as being a proven technology for decontaminating gases contaminated with a "wide" variety of hydrocarbons. Several alternate design criteria were discussed. The authors discussed a patented design that has potential for removing various volatile organics from contaminated gas streams. Neff (1990) compared the cost of treating a hydrogen sulfide 
contaminated gas using a biofilter with various other physical/chemical treatment technologies. He concludes that the biofilter's annual cost was approximately half of the cost of the cheapest abiotic technology.

251. Lith (1989) presents more information on the design of an air phase biofilter. Methyl-formate was treated at a mass rate of $500 \mathrm{~g} / \mathrm{m} 3 / \mathrm{hr}$. Lith points out the importance of humid conditions in the biofilter to optimize microbial activity. Ottengraf and van den Oever (1983) presented the results of bench-scale studies evaluating the feasibility of removing toluene, butyl-acetate, ethyl-acetate, and butanol. Gas velocities of 0.84 and $14.8 \mathrm{~cm} / \mathrm{sec}$ were evaluated. Various biokinetic constants were determined and removal was achieved. The authors conclude that biotreatment of contaminated gases is feasible and that this promising technology should be further studied. Kampbell et al. (1987) evaluated biofilters for removing a variety of nonchlorinated aliphatic hydrocarbons and TCE. They conclude that biofiltration was feasible and that biotreatment of contaminated gases had potential to be cheaper than conventional treatment methods. They state that further work must be performed to optimize system design and performance. Personal discussions with one of the authors indicate that the size requirement of the unit would be restrictive in terms of implementation (personal communication with Wilson 1990). However, with more work, the system could be optimized.

252. Two commercial vendors were identified as having units that could be used for treatment of contaminate gases (Biofiltration, Inc. 1990; . ClairTech, Inc. 1990). Unfortunately, there is little or no field experience on the performance of these systems of complex organic compounds.

\section{Summary of findings}

253. In summary, the application of biological processes for treatment of contaminated gases is technically feasible. Research efforts should be directed toward a better understanding of the microbial communities associated with contaminant uptake and degradation. Once a firm understanding of these concepts is obtained, then optimization can take place resulting in a fieldable technology that has potential for significant cost savings. The literature reviewed indicated feasibility, but little is known as to the practicality of this process. 


\section{Introduction}

254. This part describes the general approach that will be used during the bench-scale phase of this program. It is proposed that at the beginning of each fiscal year, a detailed study plan will be submitted for each work unit. At the end of each fiscal year, a yearly progress report will be drafted summarizing the results and conclusions of the year's activities.

255. Several research areas have been identified for evaluation on the bench scale. Each research area has several phases of study associated with it. The phases will be approached by performance of a series of tasks. The research areas for this program are:
a. Geotechnical considerations.
b. Pretreatment technologies.
c. Additives application.
d. Biological systems.
e. Adsorption/desorption.
f. Regulatory issues.

\section{Advisory Committee}

256. An advisory group, not inclusive of all experts in the field of bioremediation, gathered at the US Army Engineer Waterways Experiment Station (WES) by invitation on August 14 and 15, 1990, to review the first draft of the technical approach to this program. A list of attendees of the meeting is presented as Appendix $B$ of this document.

257. Many good and constructive comments were received. These comments were incorporated into the drafting of the technical approach presented in this document. An annual meeting of an advisory group will be conducted for bridging key knowledge gaps identified earlier in this document. Appendix $C$ presents a summary of the minutes of the August 1990 meeting.

\section{Analytical Chemistry}

258. Throughout the subsequent sections of the technical approach, various chemical analyses will be discussed as being performed as part of a 
study task. This section presents the chemical methods that will be used to analyze for the various analytes of concern.

a. Category I. Organic Compounds - Base neutral/acid extractables, straight chain aliphatics, volatile organic compounds, explosives.

b. Category II. Gross Pollutants - Biochemical oxygen demand (BOD), chemical oxygen demand (COD), total organic carbon (TOC), total petroleum hydrocarbons (TPHs).

c. Category III. Inorganic Compounds - Metals.

d. Category IV. Miscellaneous Analyses - Nutrients, solids, cation exchange capacity (CEC), $\mathrm{pH}$, temperature, oxygen.

e. Category V. Intermediates - Library searches, identification of potentially identifiable compounds (PICs).

\section{Analytical Methods}

259. This section discusses the groups of analyses that will be performed during this research program.

\section{Organic Compounds}

260. The methods used in analyzing the target organic compounds for this program are listed in Table 1 . Table 1 lists the various matrices that will be analyzed and the respective analytical procedure that will be followed. Also listed is the instrumentation type and brand name used in the analyses of the various organic compounds.

\section{Gross Pollutants}

261. The methods used in analyzing the gross pollutants for this program are listed in Table 2. Table 2 lists the various matrices that will be analyzed and the respective analytical procedure that will be followed.

\section{Inorganic Compounds}

262. The methods used in analyzing the inorganic compounds for this program are listed in Table 3. Table 3 lists the various matrices that will be analyzed and the respective analytical procedures that will be followed. 
Also listed is the instrumentation type and brand name used in the analyses of the various inorganic compounds.

Miscellaneous Analyses

263. The methods used in analyzing the miscellaneous analytes for this program are listed in Table 4 . Table 4 lists the various matrices that will be analyzed and the respective analytical procedure that will be followed. Also listed is the instrumentation type and brand name used in the analyses of the various analytes.

Intermediates

264. Gas chromatography (GC)/mass spectroscopy (MS) and GC will be used to identify chemical intermediates produced because of incomplete oxidation of the organic compounds. Potentially identifiable compounds detected on chromatographs will be identified using several chromatographic library systems. Known intermediates identified from literature reviews will also be tracked using appropriate analytical methods.

\section{Geotechnical Considerations}

265. This area of research will focus on traditional support required from the geosciences and engineering community for the proper evaluation, design, and implementation of both PAT and in situ based biotreatment systems.

266. Groundwater models describing processes such as biological growth and degradation kinetics, contaminant transport, and water flow within the aquifer will be used as a means of determining whether PAT or in situ, or possibly both, should be implemented at a site. Models are the one of the few ways we can estimate what is occurring underground in the aquifer. Models are essentially our "eyes" into the aquifer environment.

267. Other geotechnical subjects that will be investigated are the development of innovative additive injection systems. These systems could potentially result in improved biotreatment system performance and significant cost savings. 
268. The following objectives have been identified under this research area:

a. Compile, evaluate, improve, and, possibly, develop near-field groundwater models that can be used during implementation of both PAT and in situ technologies.

b. Identify and/or develop procedures for collection of undis turbed soil samples while maintaining sample integrity.

c. Modify the cone penetrometer to: (1) measure in situ dissolved oxygen (DO) concentrations, (2) collect groundwater/ soil samples to be used for chemical analysis to determine contaminant and additive concentrations and/or microbial enumeration, and ( 3 ) inject additives required for stimulation of biological activity during in situ remediation scenarios.

\section{Approach}

269. The technical approach used in this research area will be phased as follows:

a. Phase I. Near-field groundwater models.

Task I. Assessment of existing models.

Task II. Modify existing models.

Task III. Development of new model.

b. Phase II. Subsurface sample collection device.

Task I. Develop capability.

Task II. Collection of soil samples.

c. Phase III.

Task I. Additive injection.

Task II. Subsurface sampling.

Task III. Emplaced penetrometer rod removal.

Phase I. Near-field groundwater models

270. The approach for obtaining proper modeling support will be accomplished depending on the utility of existing model(s) for evaluation, design; and operation of in situ bioremediation strategies. For sake of discussion, the state of utility of the models will be classified into three scenarios. The first scenario is that existing models that may be sufficient for use as a site near-field groundwater model will be evaluated. If the existing model(s) is deemed acceptable, then no further efforts will be made on this work effort except for establishing familiarity prior to pilot- or full-scale evaluations. 
271. The second scenario is that existing models are deemed inadequate as is. The feasibility and benefits from modifying or manipulating existing models to perform the required tasks will be evaluated.

272. The third and final scenario is that modifications to existing model(s) are deemed infeasible; therefore, an adequate model will be developed.

273. The development of appropriate groundwater model(s) for use in supporting field implementation will be approached as discussed below:

Task I. Assessment of existing models

274. A thorough assessment of existing models will be performed. If it is determined that existing models can be used without major modifications, the models will be obtained by WES. Once the models are obtained, WES will gain familiarity with the model(s). Full modeling capability will be in place prior to needed modeling support during the pilot-or full-scale studies. Task II. Modify existing models

275. If existing models are not adequate in their present state, then the possibility of modifying the model(s) will be evaluated. The model(s) must be flexible enough to incorporate new information and/or algorithms as this information is available from the other work units in this program. Requiring this task is very likely to establish proper modeling support capabilities.

Task III. Development of a new model(s)

276. If the existing models are inadequate, a new model development may be beneficial. This work effort will be discussed in a separate work plan should pursuing this issue be necessary.

Phase II. Subsurface soil collection device

277. A subsurface collection device is required for the proper collection of samples used during the course of this research program. This requirement will be met through completion of the following tasks.

Task I. Development capability

278. WES will develop the capability for collecting in situ soil samples while maintaining microbial and chemical sample integrity. This capability will be obtained by evaluating existing equipment or through design and construction of new equipment. This sampling system must be capable of: (a) being sterilized, (b) collecting undisturbed samples at various depths, and (c) anaerobically extracting soil samples. This system will be used to collect soil samples for use in this program. 
Task II. Collection of soil samples

279. The sampling device will be used to collect soil samples, both saturated and unsaturated, for use in other research areas in this program. Field use of the unit will serve as validation of the unit's capability. Modification of the unit will probably take place as the unit is used.

Phase III. Application potential of the cone penetrometer

280. The cone penetrometer will be evaluated for use as an applications tool for implementing in situ bioremediation. The proposed applications of the cone penetrometer to be evaluated in this phase of study is listed and discussed below.

Task I. Additive injection

281. The potential for using the cone penetrometer to inject additives required for establishing biologically actives zones in the subsurface will be evaluated. The injection capacity of various available rods will be estimated and compared with the injection capacity of wells and trenches. If the injection capacity of the penetrometer system is deemed technically and economically feasible, then further development and evaluation of the system will be performed. The potential for use of larger diameter rods in the existing penetrometer equipment will also be evaluated. Once the penetrometer injection system has been developed, then the system will be field tested at an appropriate site that has not yet been selected.

Task II. Subsurface sampling

282. The potential for use of the cone penetrometer system for use in the collection of groundwater and soil samples from saturated and unsaturated zones being biologically remediated will be evaluated. These samples will be used for determining if the proper conditions required for biological remediation are being maintained in the subsurface. Note that this sampling capability is for small samples and should not be confused with the sub-surface sampling device discussed in Phase II.

283. Existing equipment will be modified with prototype equipment capable of collecting analytical quality groundwater samples. Designs for modification of the existing equipment for collection of subsurface soil samples will also be drafted and prototype equipment constructed then installed. Part of the development and evaluation process of this equipment will include verification of the sample collection capabilities of the penetrometer system in the field at an undetermined site. 
Task III. Emplaced

penetrometer rod removal

284. Development of a method to remove emplaced rods from soils will be needed. To accomplish this, the existing penetrometer rods may have to be modified to improve the capability of the penetrometer for these new application ideas. The new designs will eventually be evaluated at a test well field to be determined at that time. If deemed technically or cost prohibitive, this work effort will not be pursued beyond the design or developmental stage.

\section{Pretreatment Technologies}

Research Area Objectives

285. The objective of this research area is to evaluate chemical oxidation treatment technologies as a pretreatment technology prior to biological treatment. It is believed that chemical oxidation may be capable of converting contaminants that are biologically difficult to degrade into easier to degrade chemical intermediates of incomplete chemical oxidation. Mechanisms to improve the degradability of compounds include dehalogenation, ring cleavage, and removal of other substitutional groups such as nitro and hydroxyl groups.

Approach

286. This research area will investigate TCE and explosives as target contaminants. The other target contaminants in this research program are considered biodegradable to the extent that pretreatment is probably not cost effective.

287. Focus will be placed on controlling the oxidation process to convert the contaminants into easier to degrade intermediates. These intermediates will then be treated in a bioreactor after pretreatment.

288. Pretreatment processes to be evaluated are:

a. Chemical oxidation.

b. Chemical oxidation with UV catalyzation.

c. UV irradiation (photolysis). 
289. All of the above listed processes will be considered oxidationbased processes. A brief description of the three proposed pretreatment technologies that will be evaluated is presented below:

a. Chemical oxidation - This process uses strong oxidizers, such as hydrogen peroxide and ozone, to oxidize contaminants into environmentally safe inorganic species. The advantage of this process is that it can cleave aromatic rings and remove substitutional groups. The major drawback of the process is that, in some cases, chemical intermediates that are difficult to oxidize are produced. Many of these intermediates produced during incomplete chemical oxidation are easy to degrade biologically, whereas many of the parent compounds are difficult to degrade biologically. It is theorized that combining the two technologies (chemical oxidation and biological treatment technologies) may be the most efficient method of treating contaminated groundwater.

b. UV/Chemical oxidation - This process is very similar to chemical oxidation with the exception that UV light is added for the following reasons: (1) to increase the energy level of the compound (if the compound is UV sensitive), (2) the UV degrades the chemical oxidizer into hydroxyl radicals (a very strong oxidizing species), and (3) to utilize the synergistic effect of both UV and oxidizer to degrade the contaminants.

c. Photolysis - This process utilizes the destructive effects of UV irradiation on the chemical bonds of organics to destroy them. Photolysis is one of the primary destructive forces of xenobiotic compounds in the environment (especially in the atmosphere). Not all contaminants are fully destroyed using photolysis; however, the process can be useful in the deduction of refractory compounds into more biodegradable compounds.

290. The evaluation of these processes will be approached as follows:

Task I. Determination of reaction kinetics

291. Reactors ( 1 to $10 \ell$ in volume) will be used to determine chemical intermediate formation kinetics during oxidation of TCE, TNT, and RDX. A11 processes will be evaluated using the same reactors. The various intermediates of the oxidation reactions will be identified and target intermediates selected based on information on the biological degradation potentials presented in literature. The point during the reaction where the reaction can be halted resulting in the production of the target intermediate that is potentially easier to treat biologically will be determined. Intermediate formation kinetics will be described using simple mathematical relationships so that influents of varying strengths can be treated with minimal effort. One process for each target contaminant will be selected for further optimization 
studies. Intermediates will be analyzed using GC/MS and GC. Unknown peaks will be identified if possible by matching with various chromatographic libraries.

Task II. Optimization of intermediate formation reactions

292. Various catalysts, such as Fenton's reagents, and operating conditions, such as varying $\mathrm{pH}$ and temperatures, will be evaluated for increasing the rate of intermediate formation of chemical intermediates. Identified target intermediates will be tracked using procedures selected based on the results from Task $I$.

Task III. Biotreatment of oxidation intermediates

293. A bench-scale treatment system using the selected pretreatment technology and a bioreactor will be evaluated using contaminated groundwaters as influents. The type of bioreactor that will be selected will be based on the target intermediates. Operation of the bench-scale treatment system will be based on the intermediates selected. The source of the microbial inoculum used to establish an active biomass in the bioreactor will be determined based on the target intermediate. Candidate sources include mixed liquor from an activated sludge system, commercial microbes, and microbial populations isolated from other research activities. Bioreactor performance will be evaluated based on pretreatment intermediate and gross pollutant removals from the influent. The bioreactors will be run in duplicate.

\section{Application of Additives}

294. This research area will investigate solutions to problems associated with injection of additives to BAZs. These additives will serve as sources of nutrients, EAs, and microbial inocula. Much of the information on the theoretical requirements of an active microbial community in a contaminated environment are understood. The knowledge gaps associated with additives and BAZs are mechanisms for proper application of the additives.

\section{Research Area Objectives}

295. The objectives of this research area are to: 

a. Determine the effects of different additives on the hydraulic conductivity of the aquifer.
b. Evaluate innovative oxygen sources for microbial populations in the BAZs during in situ treatment.
c. Determine potential additive sinks.
d. Evaluate innovative methods to transport required additives into the BAZs.

\section{Important Definitions}

296. The following definitions are presented to familiarize the reader with some proposed research topics:

\section{Colloidal gas amphrons (CGAs)}

297. CGAs are small microbubbles (5 to $10 \mu \mathrm{m}$ in diameter) that have the potential to be used as an oxygen source for microbial populations and to transport additives through soils having low hydraulic conductivities. CGAs are manufactured by vigorously stirring water containing a small dose of surfactant. The final product is a foamy liquid composed of many small durable microbubbles that have the consistency of shaving cream.

\section{Electrokinetics (EK)}

298. EK is a process in which water and small colloids may be transported through porous media at velocities much faster than is achievable using advective forces. This increased velocity is induced by the addition of a small electrical potential across the porous media. In some cases, charged ions and possibly colloids can be transported through porous media via pore passages at velocities faster than the pore water.

\section{Additives}

299. Additives are chemicals that are added to BAZs to enhance the biotreatment process during remediation activities.

300. Additives to be evaluated during this program are listed and discussed below:

\section{a. Oxygen sources.}

The successful delivery of oxygen to aerobic microbial populations in the subsurface is a crucial step toward the development of viable in situ biological treatment systems. The application of the following candidate oxygen sources into saturated soil systems will be evaluated:

\section{(1) Hydrogen peroxide.}

(2) Air-enriched water. 
(3) Oxygen-enriched water.

(4) CGAs composed of air or pure oxygen.

b. AEAs .

Some microbes have the capability of utilizing chemical species other than oxygen as their EA required for contaminant oxidation. These chemical species are commonly referred to as AEAs. AEAs potentially are easier to transport through soil systems than oxygen sources because they are more soluble and much less reactive than oxygen sources. The following AEAs will be evaluated in this study phase:
(1) Nitrate.
(2) Manganese-II.
(3) Iron-II.

c. Nutrients.

Nutrients are required for the establishment of BAZs in soil systems. Therefore, the fate of nutrient species during transport through soil systems will be evaluated. Three nitrogen and three phosphate compounds will be evaluated. Inorganic compounds containing ammonia and/or phosphate that will be used in this study will be selected as part of this task. Selection will be made on both a technical and economic basis.

Approach

301. The technical approach used in this research area will be phased as follows :

a. Phase I. Selection of test soils.

b. Phase II. Effect of additives on soil fabric.

c. Phase III. Batch studies.

Task I. Hydrogen peroxide.

Task II. Oxygen.

Task III. Nutrients.

Task IV. AEAs.

d. Phase IV. Abiotic fate of additives in soils systems.

e. Phase V. Innovative transport mechanisms.

Task I. CGAs.

Task II. Gas enriched CGAs.

Task III. Microbe usage of CGAs.

Task IV. CGA transport of microbial inocula.

Task V. Electrokinetically induced transport.

Task VI. CGA and CGA/EK transport of microbes.

Phase I. Selection of test soils 
302. The soils used in this research area will be selected based on various chemical and physical characteristics of the soils. Eight soils will be evaluated in this research area. These soils include:

a. Two soils, one with high and one with low total iron and manganese concentrations, but similar classification according to the United Soils Classification System (USCS).

b. Two soils, one with high and one with low calcium concentrations, but with similar USCS classification.

c. Two soils, one with high and one with low organic carbon concentrations, but similar USCS classifications.

d. Two soils, one with high and one with low CEC, but similar USCS classifications.

e. Cleaned sand.

303. Soils will be collected as part of this phase. All soils will be characterized in terms of $\mathrm{CEC}, \mathrm{pH}$, USCS classification, sodium, potassium, calcium, iron, manganese, and TOC.

Phase II. Effect of additives on soil fabric

304. Permeameter testing will be performed because, as reactions and/or adsorption of the additives occur within the soil matrix, significant changes in soil fabric, and thereby hydraulic conductivity, can occur. If significant changes in hydraulic conductivity does occur, the ability and energy required to transport the additives through the soil increases dramatically.

305. The effect of the various additives on the hydraulic conductivity of the three soil types will be evaluated in flexible wall permeameters. Test permeants will be prepared by mixing tap water with the appropriate compounds. Permeants containing low, medium, and high concentrations of the additives will be evaluated using duplicate permeameter cells for each concentration. Changes in hydraulic conductivity will be measured and plotted against the number of pore water volumes permeated. A duplicate set of permeameters will be permeated with a bromide tracer to determine hydraulic characteristics of the permeameter. Additive breakthroughs will be monitored and compared with the breakthrough of the conservative tracer.

Phase III. Batch studies

306. Batch studies will be used to determine the reaction/adsorption characteristics of the additives with the various soil types. The approach used for each additive will vary depending on the additive; therefore, the 
additive batch studies will be discussed independently based on the individual additives.

Task I. Hydrogen peroxide

307. Various-soil constituents that react with hydrogen peroxide will be evaluated as potential hydrogen peroxide sinks. Soils will be completely characterized chemically and physically. Batch systems composed of soil and water containing various concentrations of hydrogen peroxide will be mixed in 250-ml plastic sample bottles on recipicating shaker tables. At test times of $10,20,40$, and $80 \mathrm{~min}$, the concentration of hydrogen peroxide will be measured from duplicate bottles. The liquid-to-solid ratio in the bottle will be 4:1 on a by-weight basis. The data from this task will be used to estimate the degradation rate of hydrogen peroxide in soils of varying chemical and physical composition. Hydrogen peroxide will be analyzed for using a colormetric method currently under development by WES and Mississippi State University.

Task II. Oxygen

308. The oxygen uptake rate (OUR) of the various soils will be determined in batch soil slurry/oxygenated distilled water systems. A liquid-tosolid ratio of $4: 1$ will be used. The batch studies will be performed in a BOD glass bottle with a dissolved oxygen plastic membrane probe inserted into the bottle. Oxygen uptake will be determined by measuring the dissolved oxygen concentrations every 5 min over a 1 -hr period. This task will be used to estimate the various oxygen uptake rates of the soils.

Task III. Nutrients

309. Batch adsorption isotherms will be used to determine the fate of solubilized nutrient sources with the various soils. These studies will be performed similar to batch adsorption tests performed during evaluation of activated carbon. The information from the batch adsorption tests will be used to determine a partitioning coefficient between the soil and the nutrients. It is assumed that the majority of the nutrients lost will be due to adsorption of the nutrient sources to the soil. These studies will be performed similar to activated carbon isotherm studies using 250-ml plastic bottles.

310. Initially, equilibrium times will be determined by measuring nutrient concentrations in duplicate bottles containing a liquid-to-solid ratio of $10: 1$ at test times of $1,2,4,8,24$, and $48 \mathrm{hr}$ of agitation. The aqueous phase will be distilled water with nutrient concentrations to be 
determined at the initiation of testing. Soil nutrient concentrations will be determined prior to testing. A recipitating table will be used to provide agitation. Equilibrium time will be determined by plotting aqueous phase nutrient concentration versus test time. The test time that correlates to a constant aqueous phase nutrient concentration will be considered the equilibrium time.

311. Once an equilibrium time has been determined, data required to develop the adsorption isotherm will be generated. Liquid-to-solid ratios of $100: 1,50: 1,25: 1,10: 1$, and $5: 1$ will be agitated for in duplicate bottles for period time equal to the equilibrium time. A plot of solid phase versus aqueous phase concentrations will be developed. These plots are commonly known as adsorption isotherms. A partitioning coefficient for each nutrient source will be estimated from the adsorption isotherms.

312. Nutrient sources with the lowest adsorptive characteristics will be considered optimal. Also, because soils of varying chemical composition will be evaluated, the chemical constituent that may prove most detrimental to each nutrient source in terms of chemical loss will be determined.

Task IV. AEAs

313. The same approach used in the nutrient sources will be used to evaluate the sorptive characteristics of the various AEAs; however, it is believed that chemical reactions may also serve as a significant AEA sink. One deviation from the above discussed protocol is that AEA soil concentrations will be determined analytically to complete a mass balance of the AEA. The soils will be separated from the aqueous phase by centrification prior to chemical analysis.

Phase IV. Abiotic fate of additives in soil systems

314. The results of this task will be used to verify the results of the batch studies (Phase III). From Phases II and III, four of the eight soils, two nitrogen and two phosphate compounds, and all three AEAs will be evaluated in this phase.

315. This work will be performed in 6 -ft-long by 6 -in.-diameter columns with sampling taps located every 6 in. of length. The size of the columns may change based on the hydraulic conductivity of the various soils. All column tests will be operated in an up-flow mode and run in duplicate.

316. The schedule for sample collection will be collected based on the hydraulic conductivity of the soils. Flow and volumetric outflow readings 
will be collected periodically to determine if changes in hydraulic conductivity is occurring as predicted from the permeameter tests. Changes in head loss across the columns will be monitored by measuring the pressure differential across the column using pressure gauges located on both ends of the column. High pressure, low flow positive displacements pumps will be used to force permeants up through the columns.

317. Numerical estimation methods describing loss over distance will be verified. The information obtained during the column studies will be used to "fine tune" these methods. The value of this task is to develop a predictive capability to optimize location of injection systems such as injection wells based not only on the physical characteristics of the aquifer, but also on the chemical composition of the aquifer soils.

318. This work effort is especially important for evaluating the degradation kinetics of hydrogen peroxide in soils. The constituents in soils that cause hydrogen peroxide degradation will be determined. Constituents such as iron complexes may serve as Fenton's reagents and other cations such as nickel that are known to initiate the degradation of hydrogen peroxide into hydroxyl radicals, oxygen, and water. Methods for inducing degradation of hydrogen peroxide will be investigated, because in some cases such as clean sands, hydrogen peroxide may be too conservative to allow for the formation of enough oxygen for the microbial populations to utilize (assuming that microbes cannot directly utilize hydrogen peroxide as an oxygen source because of chemical limitations and/or toxic effects). The experimental approach used for evaluating the ability of microbes to utilize hydrogen peroxide directly as an oxygen source will be described later in this document.

319. The potential for the gas-enriched permeants to cause air or gas binding in porous media will be investigated during the permeameter tests; however, the degree of binding occurring in the columns will also be evaluated by monitoring any changes in column hydraulic conductivity.

320. Microbial populations will be enumerated before and after performing the column tests to determine if any change in hydraulic conductivity may be due to blockage of the pore channels by microbial growth.

Phase V. Innovative transport mechanisms

321. Two innovative transport mechanisms have been identified as having a high potential for improving the state of the art in additive application. 
Task I. CGAs

322. The transport of oxygen using CGAs transported by advective means (bulk flow) will initially be evaluated using large rigid wall permeameters (1 ft long by 4-in. diameter) to monitor changes in soil hydraulic conductivity and the hydraulic head required to transport the CGAs through various soils. The effect of surfactant dosage and CGA diameter on the hydraulic gradient required to move the CGAs through soil will be evaluated using flexible wall permeameters. Once transport and head losses are determined using the rigid wall permeameters, transport will be evaluated using a large soil flume used to simulate an aquifer system. The overall benefit of CGAs will be evaluated by the increase of available oxygen to microbial populations.

Task II. Gas-enriched CGAs

323. The potential for generation of CGAs containing oxygen and/or methane will also be evaluated. A system will be designed to manufacture CGAs filled with oxygen and methane. Optimal gas solutions containing appropriate ratios of both oxygen and methane will be investigated for use in producing a CGA containing both gases. A CGA enriched with both oxygen and methane is an attractive method for transporting methane and oxygen to methanothrophic bacteria used in degrading chlorinated solvents in situ. Transport will be evaluated using the rigid wall permeameters and the soil flume.

Task III. Microbe usage of CGAs

324. The ability of microbes to degrade MEK in a soil system using CGA encapsulated air and/or oxygen as an oxygen source will be investigated. This evaluation will be first performed in aqueous phase bioreactors (chemostats). The ability of microbes to utilize CGAs in soil systems will be evaluated using saturated soil columns (rigid wall permeameters) filled with MEKcontaminated soil. MEK degrading microorganisms from the groundwater microbiology research phase of this program will be used. These studies should determine if microbes can utilize CGAs directly or if degradation of the CGA into oxygen must first occur prior to microbial utilization. If CGAs cannot be used directly, changing the surfactant used in the production of CGAs or surfactant dose will be investigated.

Task IV. CGA trans port of microbial inocula

325. The possibility of using CGAs to transport a microbial inoculum and nutrients through porous media will be investigated. This work effort will be performed in the large permeameters. This is very basic research; 
therefore, the technical approach used in this phase of study will be based on the progression and successes of the research. CGA-enhanced transport will be compared with hydraulically induced transport and system controls that will be composed of permeameters with no transport mechanism induced. Transport will be evaluated through enumeration of microbial populations along the one-dimentional flow axis within the permeameters.

326. Once the above-discussed work has been completed, the transport of CGAs through porous media will be evaluated using a large soil flume. Degradation rates and oxygen production will be determined by the generation of oxygen and CGA profiles. This work will be used to "fine tune" CGA formulations because CGAs must not be too conservative nor quick to degrade. CGAs must be able to produce appropriate available oxygen profiles along the length of flow.

327. Compatibility of surfactants used to formulate the CGAs with microbial populations will be evaluated using surfactants that are considered nontoxic to microbes based on available information. However, after the results of the sorption studies using various surfactants to increase contaminant solubilization are available, the potential for use of these surfactants to produce physically acceptable CGAs (i.e., size and survivability), while also increasing contaminant solubilization rates, will be investigated. Compatibility of the selected surfactant with the selected microbes from the microbiology research efforts in terms of toxicity will also have to be verified once the microbes are selected.

Task V. EK-induced transport

328. The transport of additives through porous media using CGAs with EK-induced flow will be evaluated using permeameter systems coupled with EK electrodes. The effect of EK on the velocity of CGA transport will be compared with the breakthrough data generated from the permeability studies (Task II), which uses advective forces to transport the CGAs through porous media. This work will also be performed in permeameters containing EK electrodes. Permeameter tests in this task will be run in duplicate.

Task VI. CGA and CGA/EK transport of microbes

329. The transport of microbes through soil systems using advective transport methods, inoculum-enriched CGAs, inoculum-enriched CGAs with EKinduced transport, and transport of the microbes using EK will be investigated. These studies will be performed in small columns ( $<2 \mathrm{ft}$ long) to 
determine feasibility. The retardation (sorption) of microbes during transport through the soils will be quantified by using conservative tracers such as bromide. If deemed feasible, microbial transport will be evaluated in larger columns. Potential toxicity effects of the surfactants used to manufacture the CGAs and the electrical fields associated with EK on the microbial populations will also evaluated.

\section{Biological Systems Research Area}

Introduction

330. This area of research will address the microbiological aspects of biological treatment of contaminated groundwater, saturated soils, unsaturated soils, and gases and activated carbon bioregeneration. This research area will be divided into the following research phases:

a. Groundwater treatment.

b. Saturated soil treatment.

c. Unsaturated soil treatment.

d. Activated carbon biological regeneration.

e. Off-gas biological treatment.

331. Each topic will be discussed separately, although information gained in one topic will be used to refine the efforts used in another. Separate objectives and approaches will be discussed according to the individual phases.

332. Groundwater treatment will be associated with PAT systems. The rationale will be that once the groundwater is extracted, treatment will be performed in an aboveground bioreactor. Therefore, this topic will focus primarily on the evaluation of various bioreactors and their respective limitations.

333. In terms of in situ treatment, it is assumed that during the performance of this area of research, no limitations exist in the deliverance of required chemicals to the soil systems. In other words, the soil systems have adequate hydraulic conductivity and are homogeneous. Although these conditions are not in agreement with "real world" systems, the deviations from the stated conditions and their effects on biotreatment will be evaluated in the other research areas. 
334. The objectives of this research area are listed below:

a. To determine optimal treatment scenarios for remediation of contaminated sites.

b. To identify the limitations of the various bioreactor types.

c. To ensure all contaminants are being degraded to nontoxic and nonregulated chemical species.

d. To provide an organized protocol for evaluation of the potential of applying biotechnologies at various sites.

e. To provide pertinent information of implementation of pilotscale demonstration of the technologies.

f. To perform a mass balance around the bioreactors to determine which is the extent of biological degradation.

g. To design and evaluate bench-scale innovative bioreactor designs that will reduce abiotic losses.

\section{Approach}

335. The technical approach used in this research area will be phased as follows:

a. Phase I. Groundwater treatment.

Task I. Screening of treatment conditions.

Task II. Evaluation of bioreactor types.

Task III. Limitations of bioreactors.

Task IV. Treatment trench evaluation.

Task V. Isolation of degrading microbes.

b. Phase II. Saturated soil systems.

Task I. Slurry systems.

Task II. Static column studies.

Task III. Optimization static column runs.

Task IV. Advective column studies.

c. Phase III. Unsaturated soils.

Task I. Design of bench-scale unsaturated soil columns.

Task II. Column biological degradation studies.

Task III. Isolation of degrading microbes.

d. Phase IV. Bioregeneration of spent activated carbon.

Task I. Production of spent carbon.

Task II. Potential for carbon fines generation.

Task III. Bioregeneration of MEK spent activated carbon. 
e. Phase V. Gas streams.

Task I. Development of a cometabolic population.

Task II. Design and construction of prototype bench biofilters.

Task III. Evaluation of selected biofilter design.

\section{Phase I. Groundwater treatment}

336. The thrust of this phase of research is to evaluate the feasibility of treating contaminated groundwaters using aboveground bioreactors.

Essentially, this task will address treatment of contaminated groundwaters produced during implementation of PAT systems.

Phase I objectives

337. The objectives of this phase are to:

a. Evaluate various sources of microbial inocula.

b. Evaluate the benefits of various bioreactor designs and types.

c. Identify inappropriate end-point chemical intermediates that may prove to be resistant to biological degradation.

d. Optimize bioreactor performance.

e. Determine the limitations of the various bioreactor types.

Phase I target contaminants

338. A11 of the target contaminants (TCE, MEK, BTEX, PAH, and explosives) will be evaluated in this phase of study.

Phase I Approach

339. Various tasks will be performed during the implementation of this phase of research. The information gained in each task will be used in the subsequent task. The approach presented below will be used for all of the target contaminants.

340. Task I: Screening of treatment conditions. The objective of this phase is to determine the treatment conditions required to obtain degradation of the target contaminants in aqueous solutions and to determine the parameters and/or additives that will yield optimal treatment conditions. Optimal degradation is not necessarily the most rapid degradation rate, but the fastest degradation rate that coincides with minimal or no intermediate formation.

341. Both aerobic and anaerobic biodegradation will be evaluated. Aerobic incubation will be conducted in electrolytic respirometers to determine degradation based on oxygen uptake and shake flasks to determine degradation based on substrate removal. The anaerobic incubation will be performed in nitrogen-purged sealed bottles to ensure anaerobic conditions. The sealed anaerobic microcosms will be mixed using end-over-end tumblers or rotated on 
their sides (large glass or Teflon shot may be added to facilitate mixing). Degradation in the anaerobic microcosms will be evaluated by monitoring substrate removal and product formation.

342. Alternating aerobic/anaerobic cycling will also be evaluated for explosives because TNT seems to be better transformed aerobically, while RDX degrades better anaerobically. The frequency, length, and orientation of the cycling will be determined after the other runs have been made.

343. For all incubations, a minimum of three replicates will be prepared for each treatment to be examined. Both poisoned (abiotic) and unpoisoned controls will be included. Poisoning of the microcosms will be accomplished using mercuric chloride. Unpoisoned controls are simply groundwater added to a microcosm with other additives such as inoculum or nutrients.

344. Treatment conditions to be evaluated are listed below:

a. Various $\mathrm{N}$ and $\mathrm{P}$ nutrient levels.

b. Benefits of adding various cometabolites for the explosives studies.

c. Various sources of microbial inoculum--potential sources include activated sludge systems, commercial seeds, contaminated soils, and microbes cultured from other research studies (WES and other research institutions).

d. For aerobic systems, evaluate various DO levels.

e. For anaerobic systems, evaluate various AEAs such as nitrate, manganese and iron complexes, and carbon dioxide.

345. Various operational type parameters will be measured periodically throughout the course of testing. The extent of sampling and specific analytes to be analyzed will be determined according to the contaminant being tested. At a minimum, these parameters are:

a. Levels of the target contaminants, intermediates, and final degradation products.

b. Levels of amendments.

c. Microbes to be monitored include total heterotrophic microorganisms (aerobes or anaerobes according to the system being evaluated) and total microbes capable of using the target contaminant as a carbon and energy source; for the anaerobic systems, microbes capable of using the AEAs selected.

d. $\mathrm{pH}$.

e. DO, where appropriate.

346. Degradation kinetics will be determined based on the results of this phase of testing. This information will be verified during the bioreactor phase of study which is discussed next. 
347. Task II. Evaluation of bioreactor types. This phase of research will be used to compare the performance of attached growth systems with the performance of a suspended growth system. Seed sources for each target contaminant will be taken from Task I or from sources identified in Task I.

348. A downflow-induced draft biofilter will be used as the test attached growth system. An SBR will be used to represent suspended growth systems. Essentially the treatment condition deemed optimal will give direction to the exact reactor designs. Both bioreactor systems will be on the order of 1 to $5 \ell$ in volume. Also, both systems will be designed to facilitate proper analytical sampling. Once the exact reactor designs deemed most suitable for treatment of each of the target contaminants have been determined, studies will be conducted to establish the effectiveness of each system in treating the target contaminants.

349. Potential pathways for loss of target contaminants via abiotic means such as volatilization will be addressed by operation of poisoned controls. This information will be used to further refine the reactor systems. Analysis of reactor off-gases will also be performed to determine the loss of volatile compounds via volatilization. Waste sludges produced during operation of the bioreactors will be evaluated for parent and intermediate organic compounds.

350. The performance of the bioreactors will be monitored using a series of process analyses. These analyses are listed below:

a. DO (where appropriate).

b. AEA concentrations (where appropriate).

c. Suspended and volatile suspended solids.

d. SVI.

e. $\mathrm{pH}$.

f. Influent and effluent nutrient concentrations.

g. Influent and effluent BOD, COD, and TOC concentrations.

h. Influent and effluent cometabolites (where appropriate).

i. Influent, effluent, waste sludges, and off-gas target contaminants and identified intermediates (from literature and Task I concentrations).

351. The frequency for collection of the above-listed analytes will be based on the kinetic rates determined in Task I. At a minimal pH, SVI, temperature, DO, suspended solids, and volatile suspended solids will be analyzed daily. Nutrients, AEAs, BOD, COD, TOC, and cometabolites will be analyzed 
twice a week. Influent and effluent samples will be collected every other week and analyzed for target compounds to assess contaminant removal. Off-gas and waste sludge analyses will be run at least two times during this phase.

352. Task III: Limitations of bioreactors. The objective of this phase is to determine the limitations of bioreactor systems in terms of influent quality. It is generally accepted that bacteria can mineralize low and relatively high levels of contaminants. However, mineralization of contaminants by bacteria in flasks or plates indicates that the bacteria can survive wide concentration extremes. Operation of properly functioning bioreactors can require a much more narrow range of influent strengths. Too low influent strength can result in activated sludge that has poor settling characteristics or dramatic loss of biomass in attached growth systems. Influent with too high influent strength can approach toxicity or inhibitory levels also upsetting a properly operating bioreactor.

353. This phase of study will use both bioreactor types from Task II. One replicate of each bioreactor type will be fed influent diluted in terms of the target contaminants but not nutrients. The influent will be periodically reduced until failure in bioreactor operation is observed. The second bioreactor will be used to determine an upward limitation in influent target contaminant concentrations. The third bioreactor will be used as a control; therefore, it will be operated throughout the source of Phase III just like it was operated in Task II.

354. The same operational analyses that was performed in Task II will be performed in Task III. These analyses will be determined using the same schedule used in Task II. Bioreactor failure will be considered to have occurred when contaminant removal is essentially nonexistent.

355. Task IV. Treatment trench evaluation. A biologically active treatment trench is proposed for treatment of contaminated, unconfined aquifers that do not exceed $75-\mathrm{ft}$ depths. This system is essentially an in-place attached growth system. It incorporates the benefits of both in situ and aboveground bioreactors. Much like in situ treatment, the treatment trench requires little or no pumping to facilitate treatment. Conversely, much like a bioreactor, treatment conditions in the trench can be carefully controlled.

356. Only MEK will be evaluated in this task. If deemed feasible, treatment of the other target contaminants will be evaluated as a separate work unit. 
357. A sma1l, saturated, unconfined aquifer will be used to evaluate this concept. A small trench will be maintained in the model aquifer. Actual design of the trench will be drafted as part of this task. Expected design is that the trench will be constructed much like a bentonite-soil slurry wall. Backfill will be media used in trickling filter systems.

358. Influent will flow through the aquifer and trench at a velocity on the order of $10 \mathrm{E}-5 \mathrm{~cm} / \mathrm{sec}$. Oxygen will be supplied to the attached growth biomass via air diffusers located in the front of the trench. Nutrients will be injected via injectors located in front of the trench. The trench will be evaluated by sampling for:

a. DO.

b. Suspended and volatile suspended solids.

c. $\mathrm{pH}$.

d. Influent and effluent nutrient concentrations.

e. Influent and effluent BOD, COD, and TOC concentration.

f. Influent, effluent, waste sludges, and off-gas target contaminants and identified intermediates.

359. The frequency for collection of the above-1isted analytes will

follow the same general schedule presented in Phase III.

360. Task V. Isolation of degrading microbes. Studies will be conducted to develop a reproducible procedure for isolating and characterizing individual native microbes or microbial consortia having the ability to degrade target compounds. This research effort will be performed to further facilitate the application of biological treatment technologies at various sites. Particular emphasis will be placed on determining those tests that will be most effective in selecting organisms having degradational activity against those contaminants having a high resistance to biological treatment. Developmental work will consist of the following tasks in the order indicated:

a. Develop basal media that can be readily supplemented with the target contaminants or analogs of the target contaminants as required to isolate the microbes having the desired activity.

b. Develop a series of screening tests to assay both the spectrum and intensity of degradational activity of individual microbes or microbial consortia.

c. Develop isolated microbes or microbial consortia to survive and grow in the soil medium or PAT systems to be evaluated.

d. Develop in situ activity assays to determine the actual activity of individual microbes or microbial consortia against the target contaminants within the soil medium or PAT system. 
e. Verify the procedure through application to all of the test soils media and groundwater from appropriate field sites.

Phase II. Saturated soil systems

361. In this phase of research, all target contaminants will be evaluated. This phase will focus on the ability of microbes to degrade the contaminants using in situ treatment scenarios.

Phase II Objectives

362. The objectives of this phase of research are:

a. Identify appropriate microbes for optimum degradation of the target contaminants.

b. Determine appropriate treatment conditions conducive to the optimal performance of the selected microbes.

c. Evaluate the efficiency of various additives such as cometabolites for initiating degradation and/or decreasing contaminant degradation rates.

Phase II Approach

363. An experimental approach has been developed for the evaluation of treatment of saturated soils. The experimental approach will be divided into levels of evaluation efforts referred to herein as tasks. To minimize the amount of testing done at the pilot-scale level, the tiered approach will eliminate all but those treatment conditions most likely to prove successful in the more advanced testing. The approach progresses from a basic, broad screening method (Task I) into a much more realistic, detailed level of screening (Task IV).

364. Task I. Slurry systems. Only explosives will be evaluated at the Task I level of effort. The treatment requirements for biodegradation of the other contaminants are better understood and therefore will be initiated at the Task II level.

365. Task I Objective. The objective of Task I is to determine whether a compound will degrade in soil systems under optimal mixing conditions. High mixing conditions are believed to be achieved using soil slurry systems because of improved mass-transfer conditions.

366. Task I Approach. In Task I, soils media slurried at approximately 30 percent solids concentration with groundwater from a local well will be incubated under aerobic and anaerobic conditions in reactors that will continuously be mixed to ensure maximum contact efficiency between the contaminants, additives, and microbes. The water used to formulate the slurries for the test microcosms will contain concentrations of the target compounds at those 
equal to or slightly higher than the concentration of the target compounds measured in the site groundwater during soll sample collection activities.

367. Aerobic incubation will be conducted in electrolytic respirometers to determine degradation based on oxygen uptake and substrate removal. The anaerobic incubation will be performed in nitrogen-purged sealed bottles to ensure anaerobic conditions. The anaerobic slurries in the sealed anaerobic microcosms will be mixed using end-over-end tumblers or rotated on their sides (large glass or Teflon shot may be added to facilitate mixing, if required). Degradation in the anaerobic microcosms will be evaluated by monitoring substrate removal and product formation.

368. For both aerobic and anaerobic incubations, a minimum of three replicates will be prepared for each treatment to be examined. Both poisoned (abiotic) and unpoisoned controls will be included. Exact sampling intervals for Task I will be determined based on a series of preliminary screening tests. These screening tests will be run using a carbon:nitrogen:phosphate ratio of 100:20:5, a $\mathrm{pH}$ of approximately 7.00 , and an approximate temperature range of 20 to $30^{\circ} \mathrm{C}$. The aerobic systems will be maintained at a minimum DO of $2.0 \mathrm{mg} / \ell$. These screening tests will yield a baseline degradation rate that will be used for comparison with the other conditions; however, the conditions used in the screening tests will be repeated in Task II. Sampling intervals during the screening tests will be at $0,1,5,10,15,30,60$, and 90 days.

369. Treatment conditions to be evaluated during Task I, include several different chemical additives that may enhance degradation rates. Cometabolites, such as methane, naphthalene, phenanthrene, toluene, phenol, and other analogues of the target contaminants may be added (actual levels added will be determined based on further review of literature). Surfactants that may be added to the soil to enhance contaminant accessibility will also be evaluated. The degradation potential and possible toxic effects of the surfactants will be determined. Candidate surfactants will be selected from those surfactants found promising during the sorption phase of the saturated soil phase of study. Surfactant levels will be determined using batch equilibrium leach tests. Nutrient levels, particularly those of nitrogen and phosphorous, will be examined for their ability to support and enhance degradation activity. During the anaerobic portion of Task I, potential EAs will be evaluated. Candidate AEAs include nitrate, manganese-IV, iron-III, sulfate, and carbon dioxide. The potential utility of exotic microbe addition 
will be evaluated by the addition of various microbes that have the ability to degrade the target contaminants or compounds that are structurally similar. Some sources of the exotic microbes that may be used include commercially available microbes, microbes isolated from other studies at WES, microbes isolated from other sites, and microbes isolated by other researchers.

Finally, the effect of increasing the $N$ and $P$ levels higher than traditional values will be evaluated.

370. As indicated in the degradation section, mineralization pathways for TNT and RDX have been suggested by various independent investigators; however, many factors relevant to in situ bioremediation are poorly understood. For example, what conditions favor mineralization of TNT to innocuous products, rather than the often observed transformation that results in environmentally undesirable products? The accumulation rate, persistence, environmental effects, and ultimate fate of intermediates must be determined before initiating microbial processes that generate potentially undesirable intermediates. For example, microbial degradation of RDX produces hydrazines that are environmentally unsafe. Unless the degradation of this product can be controlled, microbial remediation of RDX cannot be successful. The importance of environmental factors such as aerobic and anaerobic conditions, temperature, $\mathrm{pH}$, and nutrient status on production and accumulation of intermediates must be determined to optimize bioremediation strategies. Before effects of intermediates upon implementation of bioremediation can be determined, the intermediates must be identified. The following procedures for identifying intermediates of microbial degradation of TNT and RDX are proposed.

371. Identification of intermediate products for the explosives will be accomplished using standard procedures in microbial physiology, biochemistry, and organic chemistry. In this process, individual microorganisms capable of degrading or cometabolizing the explosives will be isolated in enrichment cultures. Identification of intermediate compounds produced by degrading microorganisms in a normal, noncometabolic pathway will be conducted. The degrading microorganisms will be grown in pure culture using the target explosive as the sole carbon and energy source. The spent culture medium will be sampled and the compound(s) appearing in the spent culture solution will be isolated and identified. Verification of both the isolated and any other proposed compound(s) as intermediates will be carried out by growing a pure culture of the microorganism up on the compound or proposed intermediate. 
Resting cells of the organism will be obtained from this culture. These organisms will then be examined in a Warburg respirometer or similar apparatus for their ability to consume the proposed intermediate and any compounds believed to be precursors and successors to the proposed intermediate. Where appropriate, information obtained in this manner for several compounds in sequence will then be examined to determined where the compounds fit into known pathways or else used to construct a proposed degradation pathway. The pathway may be validated with the use of the parent explosive with a radioactive label and then by looking for radiolabeled intermediates and daughter products in the spent culture medium.

372. Identification of intermediate products of an explosives degradation pathway that involves cometabolism is somewhat more difficult. The initial or cometabolic step(s) occurs at the beginning of the pathway, and the microorganism carrying out this activity will not be able to degrade the resulting intermediate compound. This means that, at a minimum, a second organism must take over and carry the degradation process to completion. Occasionally, more organisms are involved. To delineate the pathway and establish the intermediate products, all of the responsible organisms must be identified and the various portions of the pathway for which each organism is responsible must be determined. From this point, the work can go on to identify and validate the intermediate compounds in the same manner as described for a noncometabolic degradation pathway in the preceding paragraph.

373. Task II. Static column studies. In this task, columns with no advection of water through the columns (hence, the name static columns) will be used to evaluate a variety of factors that may impact biodegradation. Information from the Task I explosives studies will be used to set up the Task II explosives testing. The evaluation of biotreatment in saturated soils for the other target contaminants will be initiated in this task.

374. Task II Objective. The objective of this task is to evaluate various individual treatment conditions using the static columns, which are an evaluation method similar to actual soil conditions.

375. Task II approach. Task II will be used to provide a more realistic simulation of a saturated soil system than the slurry microcosms used in Task I. This Task will use small soil columns that will be filled with the various contaminated soils. The columns will be charged with enough soil to perform one complete analysis for pertinent chemicals. For each sample interval, a duplicate set of columns will be opened and the contents analyzed. 
Therefore, for every sample point required throughout the course of testing, a duplicate set of columns will be required.

376. For evaluation of aerobic conditions, the columns will be charged with the soils; oxygenated water is then passed through the columns until saturation is reached. The water will be groundwater from a local well that is saturated with pure oxygen. Pure oxygen will allow for much more available oxygen for biodegradation to occur in the columns then if air saturated water was used (oxygen saturation using air is approximately $8.5 \mathrm{mg} / \ell$ while oxygen saturation for oxygen gas is approximately $40 \mathrm{mg} / \ell$ ). The water exiting two of the columns will be analyzed to ensure that little or no washing of the soils had occurred during the saturation of the soils.

377. For evaluation of anaerobic conditions, various AEAs will be added to the water. The AEAs to be evaluated will be discussed below.

378. Treatment conditions to be evaluated during Task II, include several different chemical additives that may enhance degradation rates. Cometabolites such as methane, naphthalene, phenanthrene, toluene, phenol, and other analogues of the target contaminants may be added. Surfactants that may be added to the soil to enhance contaminant accessibility will also be evaluated. The degradation potential and possible toxic effects of the surfactants will be determined. Candidate surfactants will be selected from those surfactants found promising during the sorption phase of the saturated soil phase of study. Nutrient levels, $\mathrm{N}$ and $\mathrm{P}$, will be examined for their ability to support and enhance degradation activity. During the anaerobic portion of Task II, potential EAs will be evaluated. Candidate AEAs include nitrate, manganese-IV, iron-III, sulfate, and carbon dioxide. The potential utility of exotic microbe addition will be evaluated by the addition of various microbes that have the ability to degrade the target contaminants or compounds that are structurally similar. Some sources of the exotic microbes that may be used include commercially available microbes, microbes isolated from other studies at WES, microbes isolated from other sites, and microbes isolated by other researchers.

379. Analyses of the samples collected from the Task II columns will be based on the target contaminants being tested and the different additives that were added to the columns. At a minimum, those analyses will include the following: 
a. Target compounds and identified intermediates (from literature).

b. Final degradation compounds.

c. Additive concentrations.

d. Direct microbial counts using acridine orange stain.

e. Heterotrophic microorganisms (aerobes or anaerobes).

f. Total microbes capable of utilizing target compounds and/or cometabolites as a carbon and energy source.

g. For the anaerobic systems, total microbes capable of using the AEAs .

h. $\mathrm{pH}$.

i. DO (where appropriate).

j. Temperature.

380. Task III. Optimization static column runs. Based on results of Task II, several optimal conditions will be combined and further evaluated during Task III using the static columns. For example, if both phosphorous (used as a nutrient) and nitrate (used as an EA) were found during Task II and have comparatively higher rates than the other test additives, various ratios of the two will be evaluated in Task III.

381. Task IV. Advective column studies. The most successful treatment conditions for each contaminant from Task III will be evaluated in Task IV. Task IV will serve as a confirmatory experimental phase to assess the true potential of the selected treatment conditions to promote degradation.

382. Task IV will use 5-ft-long by 6-in.-diameter columns operated in an up-flow mode. All runs will be run in duplicate columns. Tap water will be pumped through the columns using high pressure, low-flow, positive displacement pumps. Abiotic (poisoned) and biotic control duplicate columns will be run. The results of the additives research area will be used to select oxygen and nutrient sources.

383. Changes in system head loss will be monitored by measuring the pressure differential across the column to ensure that biofouling is not occurring within the columns. Flow rate will be monitored using small rotameter flow indicators.

384. Soil samples will be collected weekly at depths of 1,3 , and $5 \mathrm{ft}$. These samples will be analyzed for target contaminants and known intermediates. Water flowing out of the columns will be analyzed for target contaminants, nutrients, oxygen, $\mathrm{pH}$, and temperature. Microbial populations in the soils and effluent will be enumerated twice a month. Column influents and 
effluents will be analyzed for other parameters deemed pertinent based on the results from Task III.

Phase III. Unsaturated soils

385. As was the case for groundwater in saturated soils systems, the vapor phase of volatile organic contaminants held within the unsaturated soil matrix will be considered as part of the soil. Treatment of volatiles that are removed from the soil by processes such as soil venting are considered in the section of this document on the microbiology of biotreatment systems for vapors.

Phase III Objectives

386. The objectives of this phase of the research plan are to:

a. Determine whether or not a target contaminant will degrade under in situ conditions in unsaturated soils.

b. Identify appropriate microorganisms for optimum degradation of the target contaminants.

c. Determine appropriate treatment conditions conducive to optimum degradation performance by the microorganisms selected.

d. Evaluate the efficacy of additives, such as cometabolites, for initiating degradation and/or increasing degradation rates.

e. Determine if treatment conditions can be established that will encourage in situ biodegradation of a target contaminant to occur at rates acceptable for cleanup applications.

f. Design and evaluate bench-scale screening procedures to determine whether or not a compound can be degraded biologically in situ. These systems will examine microbial degradation of target contaminants within the various unsaturated soil media. The system must be capable of determining the environmental conditions required for degradation and defining the treatments required to obtain the maximum rate and extent of degradation.

Phase III Approach

387. The objectives for the three-tiered screening system for unsaturated soils are to test for the initial capability for unsaturated zone soils to support microbial degradation of each target contaminant in situ, to assess the environmental requirements of microorganisms degrading the target contaminant, and to establish the treatment conditions required to obtain the maximum rate and extent of biodegradation.

388. Task I. Design of bench-scale unsaturated soil columns, The vadose zone will be simulated at the bench scale using small bench-top columns. No one unsaturated bench-top column has been established to the point where it is believed that it can be applied without some developmental work; 
however, further investigation such as into the adequacy of systems being used in other studies will conducted.

389. If continued efforts to locate an appropriate system are unsuccessful, two column designs will be evaluated. The two candidate designs are presented below.

a. Annular ring system - This design is an annular ring system wherein three major zones are separated from each other by a system of fritted or microporous cylinders. A stainless steel outer tank holding water or a nutrient solution will form the outermost ring. The inside wall of this vessel will be either stainless steel or ceramic frit. Inside of this will be the unsaturated soil being tested. The inside wall of the unsaturated soil area will also be fritted to allow for drainage. Water or the nutrient solution in the outer tank will be allowed to seep into the soil for a certain period, and the tank will then be emptied to prevent soil saturation from occurring. A gentle stream of air will be passed up through the soil ring from the bottom to the top to maintain aerobic conditions. In another version of this, the water or solution would be placed on top of the soil with a fritted disc located between the liquid and soil phases. In this case, the soil would be supported on a second fritted disc that serves as a drain, and air would enter and leave this soil through the sides of the cylinder; the latter will also be fritted.

b. Column system - In this alternative, the vadose zone soil will be held in a vertical column, and a series of ports will be placed at approximately $7.5-\mathrm{cm}$ intervals down the length of the column and will be aligned either straight or in a helix. The ports will serve as entry points for the water or nutrient solution. A syringe pump will drive the system, but fluid will be added only in quantities sufficient to wet the soil immediately around the entry point needle. The soil column itself will be supported on a frit, and air will be gently introduced from the top of the column down with the bottom frit serving as a drain for both the soil and any excess water.

390. Either or both of the above systems will be examined for their ability to support microbial degradation of the contaminants of interest. Prototype systems will be constructed, and the better of the two (or possibly another design) will be used as the test apparatus.

391. Task II. Biodegradation studies. Vadose zone physical models used in Task III will be run in triplicate along with poisoned and true controls. Radio-labeled isotopes will be used to determine the degree of contaminant degradation biologically. Microbial enumeration and identification will be performed to assess the degree and diversity of microbial growth. Suitable nutrients and/or cometabolites will be added at levels determined in Tasks I 
and II testing to stimulate degradation at maximum levels. For either of these devices, the conditions and the parameters to be sampled will all be the same as for the saturated soils tests.

392. The impacts of the following parameters will be evaluated regarding their effect on the degradation rates of the contaminants.

a. Water content - The effect of the water content of the soils on contaminant degradation and microbial population growth rates will be evaluated. Tensiometers will be installed in the columns to measure the water content of the soils.

b. Contamination levels - Different levels of contamination of the various target compounds will be evaluated to determine if there is a lower and an upper boundary to what concentrations can be degraded.

c. Oxygen - Various air flow rates through the columns will be evaluated. Oxygen probes will be located inside the columns.

d. Soil processing - Some technologies already exists that may be used to mix soils and additives in situ. These mixing technologies will be mimicked in the laboratory, then loaded into the columns to assess the potential benefits of such activities.

393. Task III. Isolation of degrading microorganisms. This task will be performed identically as Task IV proposed in Phase I (Saturated Soils) under research area objectives, except that unsaturated soils will be substituted for saturated soils, and little, if any, work will be conducted under anaerobic conditions.

Phase IV. Bioregeneration of spent activated carbon

394. This section describes the experimental methods that will be used in the development of regeneration techniques for spent activated carbon based on biological processes.

Phase IV Objectives

395. The objectives are to determine on the bench scale, the feasibility of using biological processes to regenerate spent activated carbon. Feasibility will be based on technical and economic considerations. Objectives are to:

a. Determine if microbes are capable of degrading contaminants that are adsorbed onto activated carbon.

b. Determine the effect of the degree of contaminant loading on degradation rate.

c. Make selection of appropriate microbes effective in regeneration of spent carbon. 
d. Determine the potential for microbial clogging during and after regeneration using biological processes.

e. Determine if microbial growth in the columns may enhance the contaminant removal potential of the regenerated activated carbon.

f. Determine the potential for fines generation during bioslurry and fluidized bed treatment.

g. Determine the change in sorptive capacity of the regenerated activated carbon.

h. Evaluate a variety of extraction liquids that may extract the contaminants from the spent carbon. The contaminant/ extraction aqueous solution can either be phase separated, then the contaminants biodegraded, or degrade both the extraction fluid/contaminant aqueous solutions together.

Phase IV Approach

396. Four implementation scenarios will be evaluated for feasibility in the regeneration of spent activated carbon. These scenarios are:

a. In-bed regeneration.

b. Bioslurry regeneration.

c. Fluidized bed treatment.

d. Contaminant extraction followed by treatment of the extraction fluid using biological processes.

397. The development of biological processes for regeneration of spent activated carbon will proceed using a series of tasks. Each task will be discussed below.

398. Contaminants to be evaluated. Since the concept of using biological processes to regenerate spent activated carbon is relatively unproven, this phase will first determine feasibility. Feasibility will be established by using MEK as the contaminant. MEK will also be used to evaluate various process designs for optimal regeneration of the activated carbon. MEK was selected because it is easily biodegraded and can be adsorbed on activated carbon. After feasibility is established, bioregeneration of GAC saturated with BTEX and explosives will be evaluated.

399. Task I. Production of spent carbon. To evaluate bioregeneration of spent activated carbon, spent carbon must be generated. The methods proposed are taken from methods typically used in small GAC pilot-scale studies. Small 2-ft-long by 2-in.-diameter activated carbon columns will be set up in triplicate in the laboratory. Influent contaminated with the target compound will be pumped through the columns using a peristaltic pump. A hydraulic flux of $2.0 \mathrm{gpm} / \mathrm{ft}^{2}$ of column will be used. Breakthrough will be considered 
complete and the activated carbon spent when the effluent target contaminant concentration is equal to the influent concentration. This will be determined by periodic analysis of the effluent and influent for the target contaminant.

400. Task II, Potential for carbon fines generation. Anytime granular activated carbon is handled, there is always concern for the generation of carbon fines. This concern is also valid for this study phase. Therefore, the extent of carbon fines generation during the various methods of bioregeneration will also be a factor in the evaluation of the various bioregeneration methods.

401. Fines generation will be evaluated by rinsing a load of GAC in the columns until the GAC seems clear of fines. This will be determined by turbidity analysis of the rinse water. After the GAC is considered clean, the GAC will be handled in the various regeneration bioreactors. Handling will simulate identical conditions that the GAC will undergo doing actual regeneration. After 2 weeks of operation, the bioreactors will be turned off and the GAC rinsed. Two weeks was selected as a guess of the time required for GAC bioregeneration. Fines generation will be evaluated through turbidity measurements and suspended solids concentrations in the rinse water.

402. Task III. Bioregeneration of MEK spent activated carbon. Several potential treatment configurations have been identified and will be evaluated. The two best configurations will be used to evaluate the other contaminants. The reasoning behind using the two optimal configurations is that the actual biological degradation kinetics for each contaminant may vary. Because of this variation, different treatment conditions may be required. The proposed treatment configuration is listed and discussed below.

403. In-bed regeneration. This form of bioregeneration will be performed in the actual vessel or bed used during activated carbon treatment. The bed for this study will be the same beds used for producing the spent carbon. Once breakthrough is observed, additives including an inoculum of the acclimated consortium will be added via recirculated water. Oxygen and possibly cometabolites will also be added to the recirculation water using an in-line mixing reactor. Cometabolites will be selected based on their ability to pass through a GAC bed without significant loss because of adsorption onto the GAC. Oxygen and additive levels will be monitored in the recirculation water exiting the column. Periodically, carbon will be extracted from the column and analyzed for the target contaminant and microbial enumeration. Preregeneration and post-regeneration head loss through the column will be 
measured to determine if biofouling has significantly increased the head loss through the column. After regeneration is completed, the column will be operated using the original influent to assess the postregeneration sorptive capacity of the activated carbon.

404. Slurry reactor regeneration. A column identical to the system described above will be run until breakthrough is observed. The solid phase concentration of the target contaminant will be measured using freon extractions followed by GC/MS analyses for the volatile compounds and acetone extraction followed by HPLC analysis for the explosive compounds. The spent carbon will be removed from the column and deposited into a reactor, which will be slurried in a liquid medium containing various additives such as microbial inoculum, nutrients, cometabolites, and possibly surfactants. A gas diffuser will be located inside the slurry reactor so that oxygen and possibly methane can be solubilized into the liquid phase. Once regeneration has been determined by the disappearance of the adsorbed compounds from the carbon, the carbon will be reloaded into the column for reevaluation similar to that described above. The potential for generation of fines will be evaluated under a variety of mixing energies (if fine generation is too excessive, the economic benefit of the system is reduced).

405. Fluidized regeneration. A column will be operated until the carbon is spent; the column design will allow for the spent carbon to be fluidized in the adsorber column. The fluidized bed will be configured similar to the columns described in Task I (Production of spent carbon). Once regeneration is complete, the sorptive capacity of the column will be determined to evaluate if loss of sorptive capacity has occurred and if biofouling is significant. Also, the generation of carbon fines will be evaluated.

406. In-bed contaminant extraction. A variety of extraction solutions will be evaluated for extraction of the sorbed contaminants from the spent bed. The extraction fluid will be pumped through the spent bed after the column apparatus described in the previous sections has become spent. Carbon phase contaminant levels will be determined prior to extraction. The contaminant-ladened extraction fluid will be treated in a biological reactor set-up off-line. Essentially, absorber will serve as a concentrating step for the contaminants. The extraction fluid should have higher concentrations more fit for biological treatment than most influent carbon adsorbers receive. One particularly attractive system that will be evaluated is a system that utilizes a volatile extraction fluid, like methylene chloride, in which 
contaminants are removed by the solvent from the spent carbon. The contaminant-ladened fluid will be passed through a distillation system in which the fluid is recovered for future use, while the less volatile target contaminant is concentrated, then treated in a biological reactor. This approach will be limited to the lesser volatile compounds.

\section{Phase V. Gas streams}

407. Emphasis will be placed on thoroughly understanding the kinetics of microbial uptake of contaminants from a gaseous stream. Only TCE will be investigated in this study phase. If biological treatment of contaminated off-gases is deemed technically and economically feasible, evaluation of other compounds may be investigated under a separate scope of work.

Phase V Objectives

408. The objectives are to:

a. Determine whether or not TCE will degrade in a vapor phase treatment system.

b. Devise a cometabolic system to bring about the degradation of TCE concentrations not supporting microbial growth.

c. Determine the organic products formed during cometabolic degradation and establish a mechanism to destroy these substances.

d. Determine the need for specialized microorganisms to degrade compounds sorbed to solid supports and establish a means for promoting development of these microorganisms on the solid phase.

e. Determine there is a threshold for biodegradation of volatile organic compounds that are not cometabolized, but that are degraded as a consequence of microbial growth.

$\underline{f}$. Provide information that can be used to design and construct a pilot-scale treatment system that utilizes biological processes capable of efficiently treating contaminated gas streams from other process equipment producing contaminated gas streams.

Phase V Approach

409. This research area will be approached using the following tasks: 410. Task I. Development of a cometabolic population. Liquid phase and solid support systems will be used in initial work. Two approaches will be used to obtain a microbial population that carries out cometabolism. The first approach will use the principle that microorganisms able to grow aerobically on compounds structurally related to the target contaminant are also able to degrade organic compounds that are only attacked through cometabolism. For example, for the halogenated alkanes, microbial populations can be 
developed supported by nonhalogenated analogs of the target compound. These analogs may contain fewer halogens or other nonhalogenated substituents.

411. The second approach to develop populations active in cometabolizing the target compounds will be to favor a population that oxidizes methane, a compound that is readily destroyed by microbes that use this compound for growth. Such methane-oxidizing microbes are known to be able to destroy a number of chlorinated aliphatic compounds. The population of cometabolizing microbes will be maintained in the treatment system by alternately providing the volatile compound and the growth substrate or by providing a gas stream containing both the pollutant and a carbon source sustaining growth. The existence of a threshold and the precise threshold level will be determined by using different concentrations of test chemicals, either in the gas phase or in the soluble phase, and measuring the concentrations below which there is no detectable activity.

412. Task II. Design and construction of prototype bench biofilters. Once gas phase treatment is proven potentially implementable, various biofilter designs will be evaluated. These designs will be based on the results of the above-mentioned experimental approaches. The various designs will afford the microbial populations treatment conditions that are most conducive to the complete microbial degradation of the contaminant at an optimal rate. Several types of structural growth support media will be investigated. The candidate support media include granular activated carbon, soils, plastic media, and various wood products, such as wood chips.

413. The three best system designs will be further evaluated in terms of their abilities to treat gas streams entering the systems at increased flow rates. The intent of this task is to determine the benefits and limitations of each design. Physical characteristics of the three systems, such as head loss, treatment efficiency, and potential for biofouling to occur, will be evaluated. The best system design will be selected for future testing in the subsequent task.

414. Task III. Evaluation of selected biofilter design. The optimal design selected in Task II will be evaluated for its ability to treat contaminated gas streams of varying strengths. The intent of this task is to determine if a lower influent contaminant concentration that cannot support active biomass or require excessive cometabolite addition exists. A high limit will also be investigated to determine if a toxic level exists that can disrupt system performance because of the loss of active biomass. 
Research Area Objectives

415. The overall objective of this research area is to gain a general understanding of the adsorption/desorption processes of the target contaminants and aquifer media. The impacts of these processes on the biodegradation of contaminants and on the various biological treatment implementation scenarios will be investigated.

\section{Approach}

416. The approach used in the evaluation of sorption processes will be described in a series of phases listed below:

a. Phase I. Saturated soils.

Task I. Soil characterization.

Task II. Sorption kinetics.

Task III. Batch sorption tests.

Task IV. Sequential leaching.

Task V. Intraparticle sorption.

Task VI. Correlation between sorption properties of contaminants and soil properties.

Task VII. Column leach tests.

Task VIII. Validation of batch-based concepts.

b. Phase II. Effects of sorption on microbial degradation.

Task I. Diffusion versus degradation rate.

Task II. Sorption sites on and within soil properties.

Task III. Effects of intraparticle sorption on bioavailability.

Task IV. Effects of microbial attachment on degradation rate.

c. Phase III. Desorption enhancement.

Task I. Batch testing.

Task II. Column testing.

Task III. Film and immobile water effects.

Task IV. Operational strategies.

Task V. Compatibility with degrading microbes.

d. Phase IV. Unsaturated soils.

Task I. Bench-scale physical model design and construction.

Task II. Shake-down testing of physical model.

Task III. Simulation of contaminant transport.

Task IV. Development of predictive equations. 


\section{Phase I. Saturated soils}

417. Research will focus on determining (a) the reversibility and rate of adsorption and desorption of the contaminants of interest, (b) the importance of diffusion to bioavailability of contaminants to microorganisms, and (c) the relationship between soil properties and sorption. The data obtained will be used for estimating PAT cleanup times when the local equilibrium assumption applies and when mass-transfer limitations apply. Conditions for which mass-transfer effects are limiting to bioremediation will be identified. Empirical relationships for estimating sorption properties of contaminants of interest using minimal site-specific information on soil properties will be developed. Transient microscale processes that influence rates of in situ biodegradation will be explored.

Phase I Objectives

418. The objectives identified for this research phase are:

a. To define and quantify sorption and mass-transfer limitations on bioremediation of contaminated soils and groundwater in aquifers.

b. To minimize adverse effects of sorption processes on in situ bioremediation of saturated soils and groundwater.

Phase I Approach

419. Sorption and mass-transfer limitations of in situ bioremediation in saturated zones will be addressed in a series of batch and column experiments using selected soils and contaminants. Batch tests are needed to separate the processes associated with fluid mechanics of porous media flow from processes acting at particle-water interfaces and within particles (Nathwani and Phillips 1979; Hill, Myers, and Brannon 1988). In batch tests, parameters intrinsic to the particle-water interfaces and intraparticle pore phenomena will be determined. Batch tests will be conducted as a two phase system with water and soil. Fluid mechanics can affect processes acting at particle-water interfaces. Therefore, column tests are needed as a simple physical analog to the field. Laboratory column testing provides a means for coupling fluid mechanics with processes acting at particle-water interfaces and within particles under controlled conditions. The tests are essential to avoid the variability and lack of control that characterize the field and complicate data interpretation. Batch and column experiments will provide information on microscale- and particle-scale processes and, thus, provide a baseline for evaluation of field-scale processes. 
420. Task I. Soil characterization. Both contaminated and uncontaminated soils will be collected and characterized. Characterization will include $\mathrm{pH}$ determination, particle size distribution by using the method of Day (1956) as modified by Patrick (1958), CEC using the ammonium saturation method of Schollenberger and Simon (1945), ammonium oxalate/oxalic acid extractable iron, manganese, aluminum, and calcium (Brannon and Patrick 1985), percent organic carbon (Nelson and Sommers 1982), and contaminant concentration using USEPA standardized procedures except for explosives. Explosives will be determined according to Jenkins and Walsh (1987) and/or procedures developed by the Analytical Laboratory Group, Environmental Laboratory, WES.

421. Task II. Batch sorption tests. The apparatus and testing procedures for batch testing are more varied than for column testing (Lowenbach 1978; Conway and Malloy 1981; Conway and Gulledge 1983) because so many variables can be manipulated. Batch testing can provide information for determining whether equilibrium or nonequilibrium processes are controlling contaminant transfer from soil to solution. Batch testing procedures are sufficiently flexible to address both situations. Equilibrium batch testing, however, is similar because it is based on the assumption that equilibrium processes control soil/water interactions in the field environment. The time required to reach equilibrium or steady-state adsorption is usually rapid, but desorption steady state for some contaminants is very slow. Batch desorption, or leaching, offers an advantage in testing over column testing by decreasing testing time. Renewal rate of leachate at the solid surface in a batch test is virtually infinite compared with renewal rate in a column. Therefore, batch tests can be used to determine particle scale adsorption/desorption processes, to rapidly identify the processes controlling soil/contaminant interactions, and to infer the long-term leaching response of contaminants.

422. Task III. Sorption kinetics. Batch kinetic testing for adsorption and desorption will be conducted to determine the time required to reach steady-state sorption on particle surfaces. Sorption data from batch experiments indicate an initial phase of rapid uptake on the order of minutes to hours followed by an extended period of slow uptake (Karickhoff 1980; McCall and Agin 1985; Oliver 1985). Results will be used to evaluate the local equilibrium assumption.

423. Task IV. Sequential leaching. Sequential batch leaching consists of challenging soil solids with successive aliquots of water (Myers and Brannon 1988). Solid and aqueous phases are separated by centrifugation/ 
filtration between leaching cycles. The contact time required to reach steady state determined in batch kinetic testing is used. Leachates are chemically analyzed and plots of solid phase concentration versus aqueous phase concentration are prepared. The plots yield desorption isotherms from which distribution coefficients can be obtained. The plots also yield information on the linearity of desorption and the relative significance of the irreversibly sorbed fraction. This information is needed to properly formulate the sorption term in transport equations of sorbing solutes and to determine the longterm leaching responses of contaminants.

424. Task V. Intraparticle sorption. The time for elimination of intraparticle contamination using PAT technology may be years, because diffusion is slow and is retarded by sorption to walls of minute pores in soil particles (Brusseau and Rao 1989). Furthermore, contaminants in intraparticle pores may be inaccessible to microorganisms if the pores are smaller than the microorganisms or too tortuous for them to traverse. Batch adsorption/ desorption tests conducted with water will be followed by quantification of contaminant residing in intraparticle pores. Unextractable contaminant will be quantified by complete combustion of soil residue and recovery of ${ }^{14} \mathrm{CO}_{2}$. Mass balance of tests will also be determined using radiolabeled contaminant. Gas liquid chromatography and/or high performance liquid chromatography will be used to further characterize contaminants when warranted. Results will be used to evaluate bioavailability of contaminants to microorganisms and extractability of contaminants in PAT technology.

425. Task VI. Correlation between sorption properties of contaminants and soil properties. Sorption coefficients from the sequential leach tests will be correlated with soil properties. Many empirical relationships for estimating soil-contaminant sorption coefficients have been published (Hance 1967; Chiou, Peters, and Freed 1979; Karickhoff, Brown, and Scott 1979; Karickhoff 1980; Schwarzenbach and Westal1 1981; Chiou, Porter, and Schmedding 1983). Griffin and Roy (1985) critically reviewed the literature on predicting sorption coefficients using empirical relationships, and Lyman, Reehl, and Rosenblatt (1990) provide example calculations and additional guidance on using available relationships. The available relationships include correlations of a chemical property (octanol-water partitioning coefficient or aqueous solubility) to a soil property (organic carbon content). Most of these relationships, however, focus on pesticides and nonionic organic chemicals. Pennington and Patrick (1990) showed that soil sorption of TNT correlates with 
soil CEC. CEC may, therefore, be as important as or more so than soil organic carbon in the sorption of this explosive. Empirical relationships for predicting sorption of contaminants of interest as a function of soil properties will be developed. The relationships to be developed in this study are anticipated to be contaminant dependent and dependent on one or more of the following soil properties: organic carbon, CEC, percent sand, silt and clay, and concentration of iron, aluminum, and manganese. Empirical results can be used to make a priori estimation of soil sorption coefficients using minimal information about the soils at candidate sites for bioremediation.

426. Task VII. Column leach tests. The column testing described below is designed to use batch concepts to interpret column behavior. Beginning with the local equilibrium assumption, appropriate concepts for evaluation of the feasibility of bioremediation technology will be developed (Hill, Myers, and Brannon 1988; Myers and Brannon 1988).

427. Task VIII, Validation of batch-based concepts. Pore fluid velocity is a key parameter for design and operation of field bioremediation systems. Therefore, elution curves from continuous-flow column tests conducted at various pore fluid velocities will be compared with curves predicted from the batch tests described above. If predicted and observed elution curves agree, the conclusion may be reached that interphase transfer of contaminant to pore fluid has been adequately described. If not, conceptualizations other than those investigated in the batch tests may be needed.

Phase II. Effects of sorption on microbial degradation

428. Two modes of sorption potentially affect microbial degradation of contaminants: sorption of the contaminant to soil components and sorption of degrading microorganisms to soil components. The mechanisms and ramification of these two sorption modes are discussed under research focus below.

\section{Phase II Objectives}

429. The objectives of Phase II are to:

a. Determine the significance of adsorption of contaminants on implementation of in situ bioremediation.

b. Determine the significance of adsorption of microorganisms on implementation of in situ bioremediation.

Phase II Approach

430. This phase will be approached by completion of the tasks discussed below. 
431. Task I, Diffusion versus degradation rate. To determine whether the diffusion rate of the contaminant of interest or the microbial degradation rate is limiting to in situ bioremediation, a series of tests will be conducted. The diffusion rate for contaminants of interest will be determined from desorption kinetics testing using soils from contaminated sites under biotic and abiotic conditions. Microbial degradation rates for contaminants of interest will be determined by disappearance of substrate and appearance of degradation products.

432. Task II. Sorption sites on and within soil particles. Quantifying sorption on and within soil particles will be achieved by using radiolabeled contaminants. Easily leached fraction will be separated from fractions requiring more rigorous extraction procedures, and any remaining unextractable residues will be quantified by complete combustion techniques.

433. Task III. Effects of intraparticle sorption on bioavailability. The importance of intraparticle sorption to bioavailability will be determined by comparing diffusion rates with results of sorption studies described above. In instances where intraparticle sorption is limiting to bioavailability and bioremediation, predictive techniques will be developed to allow assessment of intraparticle sorption at any site for which specific characteristics can be easily measured. These results will be integrated with results of desorption enhancement studies to provide protocols for improving bioremediation.

434. Task IV. Effects of microbial attachment on degradation rate. Effects of microbial attachment on degradation rate of contaminants of interest will be studied using a modification of the methods of Orgam et al. (1985). Controlled laboratory studies will examine degradation with microorganisms held in solution and sorbed to soil surfaces. The influence of contaminant sorption on degradation rate with suspended and soil-attached microorganisms will also be studied (Orgam et al. 1985). Radiolabeled cells will be used to determine the location of microorganisms using modifications of procedures developed by Efroymson and Alexander (1991). Radiolabeled contaminants will also be used to assess degradation rate and extent. Pure culture studies will be conducted to assess degradation of the contaminant by microorganisms attached directly to the contaminant. Similar studies will be used to assess degradation of insoluble contaminants via microbial secretions. Phase III, Desorption enhancement

435. The research will focus on optimization of in situ bioremediation by enhancing desorption of the contaminants of interest. Agents potentially 
enhancing removal or bioremediation will be tested with soil-contaminant sys-

tems for effectiveness and with microorganisms for compatibility.

Phase III Objectives

436. The objectives of Phase III are:

a. To improve movement of contaminants through soils.

b. To increase bioavailability of sorbed contaminants.

Phase III Approach

437. This phase will be approached using the following tasks:

438. Task I. Batch testing. Facilitated transport by colloids will be investigated in batch tests by fractionation of leachate into truly soluble and colloidal fractions. This will be accomplished by using radiotracers when appropriate and by passing leachates through $\mathrm{C}^{18}$ SepPaks, which retain truly dissolved fractions. Results will be used to assess the importance of facilitated transport to bioavailability of contaminants. Cosolvent enhancement of desorption will also be investigated in batch tests. Test systems will include water/MEK and water with one or more petroleum hydrocarbons with explosives or PAHs as contaminants.

439. Transport of many organic contaminants can be increased by leaching soils with hot water, since solubility is greater at higher temperatures. Batch partitioning tests using hot water will be performed with petroleum hydrocarbons and explosives.

440. Selected surfactants and colloids will be screened for their effectiveness in mobilizing contaminants in saturated soil systems. Aqueous solutions of test chemicals will be equilibrated with test soils, or contaminated soils will be tested with the chemicals. Batch desorption tests will be conducted and the data compared with data obtained without addition of surfactants and colloids. Since surfactants may enhance biodegradation without increasing the mobility of the contaminant (Aronstein, Cavillo, and Alexander 1991), effects of surfacants on the biodegradation process will be evaluated. 441. Task II. Column testing. Column testing will be conducted to determine if extraction enhancement techniques, e.g., addition of surfactants or colloids, can minimize or eliminate sorption and mass-transfer effects in a continuous and/or pulsed flow system.

442. Task III. Film and immobile water effects. Batch testing cannot adequately simulate some important porous media phenomena. These include film and immobile water effects. Immobile water regions act as long-term reservoirs for contaminant elution because diffusion controls transfer of 
contaminant to the bulk flow of pore fluid. If pore fluid velocities are too high, water may be advected away and replaced by water up gradient with little transport of contaminant out of the soil. Films at particle surfaces can also provide significant resistances to desorption of contaminant from aquifer materials to the bulk flow of pore fluid.

443. Column tests will be conducted at various flows using tritium and other tracers to investigate the significance of film effects and immobile water regions. Some columns will be eluted with water, followed by alcohol, and then water again to investigate the hydraulic regime. Both packed and "undisturbed" soil cores will be studied at two temperatures. Effects of leaching with hot water will also be investigated. Contaminant elution curves will be used to evaluate the significance of immobile water and film effects on in situ bioremediation.

444. Task IV, Operational strategies. Once a basic understanding of the processes governing bioavailability and desorption is developed, column tests will be conducted to simulate various operational strategies for the field, e.g., continuous pumping and pulsed pumping. Continuous pumping maintains a constant hydraulic gradient, providing control over advection of contaminant. Pulsed pumping, while allowing maximum concentrations of contaminant to be extracted efficiently and reducing energy requirements and costs, may require longer remediation times. The effect of these operational strategies on cleanup will be evaluated.

445. Task V. Compatibility with degrading microorganisms, Effective desorption enhancing agents will be tested with degrading microorganisms to determine the effects of the agent on the survival and growth of the degrading microorganisms, the extent and rate of degradation, and the products formed. Phase IV. Unsaturated soils

446. Special consideration must be given to unsaturated soils, because the presence of air in the soil and the drying cycles to which the soil is subjected create sorption properties that differ from those of saturated soils.

447. The presence of air, the reduced water content, and the potential for volatilization result in significantly more complicated microscale contaminant transport processes in the unsaturated zone than in the saturated zone. Because of the complexity of the unsaturated zone, research will focus on the effects of sorption and mass-transfer limitations rather than on separate microscale processes such as intraparticle diffusion, sorption, and film 
effects as proposed for the saturated zone research. Intraparticle diffusion, sorption, and film effects are certain to be present; therefore, information obtained in the saturated soil research should be applicable, with some modifications, to the unsaturated zone.

Phase IV Objectives

448. Objectives of sorption studies in unsaturated soils are as follows :

a. To define and quantify sorption and mass-transfer limitations on bioremediation of contaminated soils in the vadose zone.

b. To minimize adverse effects of sorption processes on in situ bioremediation of vadose zone soils.

c. To evaluate the significance of volatilization in the vadose zone.

Phase IV Approach

449. Contaminant movement in the unsaturated zone above a water table aquifer will be modeled. Physical modeling will provide information on overall sorption and mass-transfer parameters in the unsaturated and saturated zones. Predicted and observed sorption and mass-transfer effects in the experimental system described below will be compared.

450. Task I. Bench-scale physical model design and construction. The physical model will be patterned after the flume used by Jubboori, Stewart, and Adrian (1974) to investigate recharge well clogging. The Jubboori, Stewart, and Adrian (1974) model has the advantage of providing a concave piezometric surface that increases the volumetric extent of the unsaturated zone over the volume achievable using a rectangular flume. The model will be constructed as a 15-deg, pie-shaped flume with approximate dimensions of 1 to $2 \mathrm{~m}$ in length and 1 to $2 \mathrm{~m}$ deep. Exact dimensions will be developed after further analysis of physical modeling requirements. Launders will be placed on each end to provide a constant hydraulic gradient. The hydraulic gradient(s) will be selected after further analysis of modeling requirements. Piezometers and tensiometers will be used to monitor water levels in the saturated zone and water content in the unsaturated zone, respectively. Sampling locations for the piezometers and tensiometers will be determined after further analysis of modeling requirements. The model will be equipped with soil gas probes that connect to a dedicated gas chromatograph. Sampling, data acquisition, and data reduction will be controlled by a microcomputer. 451. Task II. Shake-down testing of physical model. Dye tests with clean sand will be conducted to identify the principal regions of flow and 
operational problems in the physical model. The three regions of flow are anticipated to be as follows: saturated zone, a wet zone above the saturated zone, and a relatively dry zone above the wet zone. Modifications to the physical model will be made according to the needs indicated by the shake-down tests. Shake-down tests are also needed to refine the automated soil gas sampling and analysis system.

452. Task III. Simulation of contaminant transport. The model will be loaded with contaminated soil that has been mixed and compacted during loading to provide as much spatial homogeneity as possible. Analysis of soil cores from the unsaturated and saturated zones and data from the automated soil gas monitoring system will be used to determine the movement of contaminant in the model.

453. Several types of experiments will be required. Abiotic and biotic tests and tests using clean soil, as well as contaminated soil from field sites, will be necessary. Abiotic and biotic simulations are needed to separate biodegradation effects from sorption and mass-transfer effects. Clean and contaminated soil tests will provide information on how sorption and masstransfer limitations affect the rates at which soils become contaminated and the rate at which contaminants are eluted from contaminated soils. In addition, tests must be conducted for various periods of time to fully develop contaminant profiles as a function of space and time.

454. Task IV. Development of predictive equations. Information from the physical model and from saturated soil research will be used to evaluate sorption and mass-transfer limitations in unsaturated zones. Contaminant transport equations will be developed for vadose and saturated flow regions. Sorption and mass-transfer limitations will be evaluated by comparing observed and predicted contaminant distributions in the physical model. 


\section{Research Objectives}

455. The objective of this task is to identify regulations that may prevent some of the additives used in the in situ and PAT application scenarios from being injected into aquifers during remediation of contaminated

sites. This information will be used to assess the practicality of implementing biological technologies developed during this program.

\section{Approach}

456. This investigation will be focused primarily toward federal laws; however, some states with reputations for tough environmental regulations will be investigated. The outcome of these investigations will in no way hinder or limit the scope of this research program.

457. This task will be accomplished by performing a thorough review of pertinent regulations and by interviews with key regulatory personnel. 


\section{REFERENCES}

Aamand, J., Jorgensen, C., Arvin, E., and Jensen, B. K. 1989. "Microbial Adaptation to Degradation of Hydrocarbons in Polluted and Unpolluted Groundwater," Journal of Contaminant Hydrology, Vol 4, pp 299-312.

Acar, Y. B., Hamed, J., and Gale, R. J. 1990 (Jun). "Electrokinetic Soil Processing; An Emerging Technology in Waste Remediation, " Proceedings of Hazmat 90 Conference.

Aelion, C. M., Swindoll, C. M., and Pfaender, F. K. 1987. "Adaptation to and Biodegradation of Xenobiotic Compounds by Microbial Communities from a Pristine Aquifer," Applied and Environmental Microbiology, Vol 53, pp 2212-2217.

Aguwa, A. A., Patterson, J. W., Haas, C. N., and Kno11, K. E. 1984. "Estimation of Effective Intraparticle Diffusion Coefficients With Differential Reactor Columns," Journal Water Pollution Control Federation, Vol 56, No. 5, pp $442-448$.

Alexander, M. 1965. "Biodegradation: Problems of Molecular Recalcitrance and Microbial Fallibility," Advances in Applied Microbiology, Vo1 7, pp 35-80.

- 1971. "Biochemical Ecology of Microorganisms," Annual Reviews of Microbiology, Vo1 25, pp 361-392.

1977. Introduction to Soil Microbiology, 2nd ed., John Wiley and Sons, Inc., New York.

- 1981. "Biodegradation of Chemicals of Environmental Concern," Science, Vol 211, pp 132-138.

. 1985. "Biodegradation of Organic Chemicals, " Environmental Science and Technology, Vol 18, pp 106-111.

American Petroleum Institute. 1978. "Field Application of Subsurface Biodegradation of Gasoline in a Sand Aquifer," API Publication No, 4430.

Antonic, R. L. 1975. Fixed Biological Surfaces-Wastewater Treatment, CRC Press Inc., West Palm Beach, FL.

Armstrong, D. E., and Chesters, G. 1969. "Adsorption Catalyzed Chemical Hydrolysis of Atrazine," Environmental Science and Technology, Vol 2, pp 683-689, cited in Orgam, A. V., Jessup, R. E., Ou, L. T., and Rao, P. S. C. 1985. "Effects of Sorption on Biological Degradation Rates of (2,4-Dich1orophenoxy)acetic Acid in Soils," Applied and Environmental Microbiology, Vo1 49, pp 582-587.

Aronstein, B. N., Calvillo, Y. M., and Alexander, M. 1991. "Effect of Surfactants at Low Concentrations on the Desorption and Biodegradation of Sorbed Aromatic Compounds in Soils, " Environmental Science and Technology, Vol 25, pp $1728-1731$.

Arthur, M. F., O'Brien, G. K., Marsh, S. S., and Zwick, T. C. 1988 (Aug).

"Evaluation of Innovative Approaches to Stimulate Degradation of Jet Fuel in Subsoils and Groundwater," Naval Civil Engineering Laboratory.

Arvin, E., Jensen, B., Aamand, J., and Jorgensen, C. 1988. "The Potential of Free-Living Ground Water Bacteria to Degrade Aromatic Hydrocarbons and Heterocyclic Compounds," Water Science Technology, Vol 20, pp 109-118. 
Atlas, R. M. 1981. "Microbial Degradation of Petroleum Hydrocarbons: An Environmental Perspective," Microbiological Reviews, Vo1 45, pp 180-209.

Atlas, R. M. (Editor). 1984. Petroleum Microbiology, Macmillan Publishing Company, New York.

Bahr, J. M., and Rubin, J. 1987. "Direct Comparison of Kinetic and Local Equilibrium Formulations for Solute Transport Affected by Surface Reactions," Water Resources Research, Vo1 23, p 438.

Banerji, S. K. 1990. "Draft-Attached Growth Biological Wastewater Treatment Process," Draft- USAE Waterways Experiment Station, Vicksburg, MS.

Barrio-Lage, G. A., Parsons, F. Z., and Lorenzo, P. A. 1988. "Biostimulation of Trichloroethylene Biodegradation in Microaerophilic Microcosms, "Environmental Toxicology and Chemistry, Vo1 7, pp 889-895.

Bartha, R., and Atlas, R. M. 1977. "The Microbiology of Aquatic Oil Spills," Advances in Applied Microbiology, Vo1 22, pp 225-266.

Bautista, E. M., and Alexander, M. 1972. "Reduction of Inorganic Compounds by Soil Microorganisms," Proceedings of the Soil Science Society of America, Vol 36, pp 918-920.

Be11, J. P., and Tsezos, M. 1987. "Removal of Hazardous Organic Pollutants by Adsorption on Microbial Biomass," Water Science Technology, Vol 19, pp 409-416.

Benedek, A. 1980. "Simultaneous Biodegradation and Activated Carbon Adsorption-A Mechanistic Look," Activated Carbon Adsorption of Organics From the Aqueous Phase, McGuire, M. J. and Suffet, I. H. -Editors, Ann Arbor Science Pub.

Benefield, L. D., and Randa11, C. W. 1980. Biological Process Design for Wastewater Treatment, Prentice-Hall Inc.

Bengtsson, G., Enfield, C., and Lindqvist, R. 1987. "Macromolecules Facilitate the Transport of Trace Organics," Science of the Total Environment, Vo1 67, pp 159-164.

Berry, D. L., Francis, A. J., and Bollag, J. M. 1987. "Microbial Metabolism of Homocyclic and Heterocyclic Aromatic Compounds Under Anaerobic Conditions," Microbiological Reviews, Vol 51, pp 43-59.

Best, E. P. H., Dassen, J. H. A., Boon, J. J., and Wiegers, G. 1990. "Studies on Decomposition of Ceratophyllum demersum Litter Under Laboratory and Field Conditions: Losses of Dry Mass and Nutrients, Qualitative Changes in Organic Compounds and Consequences for Ambient Water and Sediments," Hydrobiologia, Vol 194, pp 91-114.

Bieszkiewiez, E., and Pieniadz-Urbaniak, A. 1984. "Effect of Benzene and Xylene on the Work of Activated Sludge," ACTA Microbiology Pollution, Vo1 33, No. $3 / 4$.

Biofiltration, Inc. 1990. "Biofiltration Technology for the Control and Treatment of Odor and VOC Emissions at Wastewater Treatment Plants," Technical Brochure, Biofiltration, Inc., Gainesville, FL.

Blaney, B. L., and Branscome, M. 1988 (Aug). "Air Strippers and Their Emissions Control at Superfund Sites," Environmental Protection Agency Report No. $600 / D-88 / 153$. 
Boethling, R. S., and Alexander, M. 1979. "Effect of Concentration of Organic Chemicals on Their Biodegradation by Natural Microbial Communities," Applied and Environmental Microbiology, Vol 37, pp 1211-1216.

Bossert, I. D., and Bartha, R. 1984. "The Fate of Petroleum in Soil Ecosys tems," in R. M. Atlas (Ed), Petroleum Microbiology, Macmillan Publishing Company, New York, pp 436-473.

- 1986. "Structure-Biodegradability Relationships of Polycyclic Aromatic Hydrocarbons in Soil," Bulletin of Environmental Contamination and Toxicology, Vol 37, pp 490-495.

Bouwer, E. J., and McCarty, P. L. 1983a. "Transformations of Halogenated Organic Compounds Under Denitrification Conditions," Applied and Environmental Microbiology, Vol 45, pp 1295-1299.

1983b. "Transformations of 1- and 2-Carbon Halogenated Aliphatic Organic Compounds Under Methanogenic Conditions," Applied and Environmental Microbiology, Vol 45, pp 1286-1294.

Bouwer, E. J., Rittmann, B. E., and McCarty, P. L. 1981. "Anaerobic Degradation of Halogenated 1- and 2-Carbon Organic Compounds," Environmental Science and Technology, Vol 15, pp 596-599.

Bouwer, E. J., and Wright, J. P. 1988. "Transformations of Trace Halogenated Aliphatics in Anoxic Biofilm Columns, "Journal of Contaminant Hydrology, Vol 2, pp 155-169.

Bowers, A. R., Gaddipati, P., Eckenfelder, W. W. 1989. "Treatment of Toxic or Refractory Wastewaters with Hydrogen Peroxide, "Water Science Techology, Vol 21.

Boyle, M. 1989. "The Environmental Microbiology of Chlorinated Aromatic Decomposition," Journal of Environmental Quality, Vol 18, pp 395-402.

Brannon, J. M., and Patrick, W. H., Jr. 1985. "Fixation and Mobilization of Antimony in Sediments, " Environmental Pollution (Series B), Vol 9, pp 107-126.

Brock, T. D. 1970. The Biology of Microorganisms, Prentice-Ha11, Inc., Englewood Cliffs, NJ.

Brunner, W., and Focht, D. D. 1984. "Deterministic Three-Half-Order Kinetic Mode1 for Microbial Degradation of Added Carbon Substrates in Soil, " Applied and Environmental Microbiology, Vol 47, pp 167-172.

Brunner, W., Sutherland, F. H., and Focht, D. D. 1985. "Enhanced Biodegradation of Polychlorinated Biphenyls in Soil by Analog Enrichment and Bacterial Inoculation," Journal of Environmental Quality, Vol 14, pp 324-328.

Brusseau, M. L., and Rao, P. S. C. 1989. "Sorption Nonideality During Organic Contaminant Transport in Porous Media," CRC Critical Reviews In Environmental Control, Vol 19, No. 1, pp 33-99.

Carberry, J. B., and Lee, S. H. 1990. "Fate and Transport of Petroleum in the Unsaturated Soil Zone Under Biotic and Abiotic Conditions, "Water Science Technology, Vo1 22, No. 6, pp 45-52.

Carpenter, D. F., McCormick, N. G., Cornell, J.H., and Kaplan, A. M. 1978. "Microbial Transformation of ${ }^{14} \mathrm{C}$-Labeled 2,4,6-Trinitrotoluene in an Activated-Sludge System," Applied and Environmental Microbiology, Vol 35, pp 949-954. 
Cerniglia, C. E. 1984. "Microbial Transformation of Aromatic Hydrocarbons," in R. M. Atlas (Ed), Petroleum Microbiology, Macmillan Publishing Company, New York, pp 99-128.

Chiou, C. T., Peters, L. J., and Freed, V. H. 1979. "A Physical concept of Soil-Water Equilibría for Nonionic Organic Compounds," Science, Vol 206, pp 831-832.

Chiou, C. T., Porter, P. E., and Schmedding, D. W. 1983. "Partition Equilibria of Nonionic Organic Compounds Between Soil Organic Matter and Water," Environmental Science and Technology, Vo1 17, No. 4, pp 227-231.

Ciriello, S., Barnett, S. M., and Deluise, F. J. 1980. Separation Science Technology, Vol 17, p 521.

ClairTech, Inc. "Biotron Brochure," ClairTech.

Conne11, W. E., and Patrick, W. H., Jr. 1968. "Sulfate Reduction in Soil: Effect of Redox Potential and pH," Science, Vol 159, pp 86-87.

Conway, R. A., and Gulledge, W. P. 1983. Hazardous and Industrial Solid Waste Testing, 2nd Symposium. ASTM STP 805, American Society for Testing and Materials, Philadelphia, PA.

Conway, R. A., and Malloy, B. C. 1981. Hazardous Solid Waste Testing: First Conference, ASTM STP 760, American Society for Testing and Materials, Philadelphia, PA.

Copa, W. M., and Meidl, J. A. 1986. "Powdered Carbon Effectively Treats Toxic Leachate," Pollution Engineering, Vol 18, Number 7.

Cord-Ruwisch, R., and Garcia, J. L. 1985. "Isolation and Characterization of an Anaerobic Benzoate-Degrading Spore-Forming Sulfate-Reducing Bacterium, Desulfotomaculum sapomandens sp. nov., " FEMS Microbiology Letters, Vol 29 , pp 325-330.

Curtis, G. P., Roberts, P. V., and Reinhard, M. 1986. "A Natural Gradient Experiment on Solute Transport in a Sand Aquifer, IV. Sorption of Organic Solutes and Its Influence on Mobility, "Water Resources Research, Vo1 22, p 2059.

Davey, J. F., and Gibson, D. T. 1974. "Bacterial Metabolism of Para and Meta-Xylene: Oxidation of a Methyl Substituent," Journal of Bacteriology, Vo1 119, pp 923-929.

Day, P. R. 1956. "Report of the Committee on Physical Analyses, 1954-1955, Soil Science Society of America," Proceedings, Soil Science Society of America, Vol 20, pp 167-169.

DeLaune, R. D., Hambrick, G. A., III, and Patrick, W. H., Jr. 1980. "Degradation of Hydrocarbons in Oxidized and Reduced Sediments, " Marine Pollution Bulletin, Vo1 11, pp 103-106.

Desai, A. J., Patel, K. M., and Desai, J. D. 1988. "Emulsifier Production by Pseudomonas fluorescens during the Growth on Hydrocarbons," Current Science, Vo1 57, pp 500-501.

DeWaters, J. E., and DiGiano, F. A. 1990. "The Influence of Ozonated Natural Organic Matter on the Biodegradation of a Micropollutant in a GAC Bed," Journal of the American Water Works Association, pp 69-75. 
Dibble, J. T., and Bartha, R. 1979. "Effect of Environmental Parameters on the Biodegradation of Oil Sludge," Applied and Environmental Microbiology, Vol 37, pp 729-739.

Dietrich, M. J., Copa, W. M. , Chowdhury, A. K., and Randa11, T. L. 1988. "Removal of Pollutants from Dilute Wastewater by the PACT Treatment Process," Environmental Progress, Vol 7, No. 2.

DiToro, D. M., Jeris, J. S., and Ciarcia, D. 1985. "Diffusion and Partitioning of Hexachlorobiphenyl in Sediments," Environmental Science and Technology, Vol 19, No. 12, pp 1169-1176.

Dobbs, R. A., and Cohen, J. M. 1980. Carbon Absorption Isotherms For Toxic Organics, USEPA Report No. EPA-600-8-023, Cincinnati, OH.

Dobrevski, I., and Zvezdova, L. 1989. "Biological Regeneration of Activated Carbon," Water Science and Technology, Vol 21, No. 1, p 144.

Donaldson, T. L. et al. 1987. "Biooxidation of Coal Gasification Wastewater Using Fluidized-Bed Bioreactors," Environmental Progress, AiCHE Vol 6, No. 4.

Edwards, N. T. 1983. "Polycyclic Aromatic Hydrocarbons (PAHs) in the Terrestrial Environment - A Review, " Journal of Environmental Quality, Vol 12, No. 4, pp 427-441.

Efroymson R. A., and Alexander, M. 1991. "Biodegradation by an Arthrobacter Species of Hydrocarbons Partitioned into An Organic Solvent," Applied and Environmental Microbiology, Vol 57, pp 1441-1447.

Emery, D. D., and Kopecky, A. L. 1989 (Dec). "In Situ Biodegradation of Creosotes and PCB's Using White Rot Fungus. 1," Presented at the Institute of Gas Technology Symposium on Gas, Oil, and Coal Biotechnology, New Orleans, LA.

Enfield, C., and Bengtsson, G. 1988. "Macromolecular Transport of Hydrophobic Contaminants in Aqueous Environments," Ground Water, Vol 26, No. 1, pp $64-70$.

Engler, R. M., and Patrick, W. H., Jr. 1973. "Sulfate Reduction and Sulfide Oxidation in Flooded Soll as Affected by Chemical Oxidants, " Proceedings of the Soil Science Society of America, Vo1 37, pp 685-688.

Fathepure, B. Z., and Boyd, S. A. 1988. "Reductive Dechlorination of Perchloroethylene and the Role of Methanogens," FEMS Microbiological Letters, Vo1 49, pp 149-156.

Fedorak, P. M., and Westlake, D. W. S. 1981. "Degradation of Aromatics and Saturates in Crude Oil by Soil Enrichments," Water. Air, and Soil Pollution, Vo1 16, pp 367-375.

Fernando, T., Bumpus, J. A., and Aust, S. D. 1990. "Biodegradation of TNT (2,4,6-Trinitrotoluene) by Phanerochaete chrysosporium," Applied and Environmental Microbiology, Vol 56, pp 1666-1671.

Fiala, E. S. 1977. "Investigations into the Metabolism and Mode of Action of the Colon Carcinogens 1,2-dimethylhydrazine and Azoxymethane," Cancer, Vol 40, pp 2436-2445.

Fliermans, C. B., Phelps, T. J., Ringelberg, D., Mikell, A. T., and White, D. C. 1988. "Mineralization of Trichloroethylene by Heterotrophic Enrichment Cultures," Applied and Environmental Microbiology, Vol 54, pp 1709-1714. 
Focht, D. D., and Brunner, W. 1985. "Kinetics of Biphenyl and Polychlorinated Biphenyl Metabolism in Soil, " Applied and Environmental Microbiology, Vo1 50, pp 1058-1063.

Fogel, M. M., Taddeo, A. R., and Fogel, S. 1986. "Biodegradation of Chlorinated Ethenes by a Methane-Utilizing Mixed Culture," Applied and Environmental Microbiology, Vol 51, pp 720-724.

Freeze, R. A., and Cherry, J. A. 1979. Groundwater, Prentice-Hall, Englewood Cliffs, NJ.

Gaudy, C. P. and Lim, H. C. 1980. Biological Wastewater Treatment, Marcel Dekker, Inc., New York.

Ghiorse, W. C., and Balkwill, D. L. 1983. "Enumeration and Morphological Characterization of Bacteria Indigenous to Subsurface Environments, " Developments in Industrial Microbiology, Vo1 24, pp 213-224.

Gibson, D. T., and Subramanian, V. 1984. "Microbial Degradation of Aromatic Hydrocarbons," in D. T. Gibson (Ed), Microbial Degradation of Organic Compounds, Marcel Dekker, Inc., New York, pp 181-252.

Goeddertz, J. G. et a1. 1988. "Offline Bioregeneration of Granular Activated Carbon," Journal of Environmental Engineering, ASCE, Vol 114, No. 5, p 1063.

Goldstein, R. M., Mallory, L. M., and Alexander, M. 1985. "Reasons for Possible Failure of Inoculation to Enhance Biodegradation," Applied and Environmental Microbiology, Vo1 50, pp 977-983.

Gotoh, S., and Patrick, W. H., Jr. 1974. "Transformation of Iron in a Waterlogged Soil as Influenced by Redox Potential and pH," Proceedings of the Soil Science Society of America, Vol 38, pp 66-70.

Gottschalk, G. 1979. Bacterial Metabolism, Springer-Verlag Publishing Company, New York.

Grbic-Galic, D. 1989. "Microbial Degradation of Homocyclic and Heterocyclic Aromatic Hydrocarbons Under Anaerobic Conditions," in J. Cooney and 0 . Sebek (Eds), Developments in Industrial Microbiology (Journal of Industrial Microbiology. Supp1. No. 4), Vo1 30, pp 237-253.

1990. "Anaerobic Microbial Transformation of Nonoxygenated Aromatic and Alicyclic Compounds in Soil, Subsurface, and Freshwater Sediments," in J.-M. Bollag and G. Stotzky (Eds), Soil Biochemistry, Vol 6, Marcel Dekker, Inc., New York, pp 117-189.

Grbic-Galic, D., Henry, S. M., Godsy, E. M., Edwards, E., and Mayer, K. P. 1990. "Anaerobic Degradation of Aromatic Hydrocarbons and Aerobic Degradation of Trichloroethylene by Subsurface Microorganisms," in Proceedings of the Meeting of the American Chemical Society. Boston. MA.

Grbic-Galic, D., and Voge1, T. M. 1987. "Transformation of Toluene and Benzene by Mixed Methanogenic Cultures," Applied and Environmental Microbiology, Vol 53, pp 254-260.

Grbic-Galic, D., and Young, L. J. 1985. "Methane Fermentation of Ferulate and Benzoate: Anaerobic Degradation Pathways," Applied and Environmental Microbiology, Vol 50, pp 292-297.

Greenhouse, G. 1976. "Evaluation of the Teratogenic Effects of Hydrazine, Methylhydrazine, and Dimethylhydrazine On Embryos of Xinopus laevis, the South African Clawed Toad," Teratology, Vo1 13, pp 167-177. 
Gregory, 0. J., Barnett, S. M., and Deluise, F. J. 1982. "Separation Science Technology," Vo1 17, p 521.

Griffin, R. A., and Roy, W. R. 1985. "Interaction of Organic Solvents with Saturated Soil-Water Systems," Open File Report No. 3, Environmental Institute for Waste Management Studies, University of Alabama, Tuscalooosa, AL.

Gschwend, P. M., and Reynolds, M. D. 1987. "Monodisperse Ferrous Phosphate Colloids in an Anoxic Groundwater Plume," Journal of Contaminant Hydrology, Vol 1, No. 3, pp 309-327.

Haines, J. R., and Alexander, M. 1974. "Microbial Degradation of HighMolecular Weight Alkanes," Applied Microbiology, Vol 28, pp 1084-1085.

Hallas, L. F., and Alexander, M. 1983. "Microbial Transformation of Nitroaromatic Compounds in Sewage Effluent," Applied and Environmental Microbiology, Vol 45, No. 4.

Hambrick, G. A., III, DeLaune, R. D., and Patrick, W. H., Jx. 1980. "Effect of Estuarine Sediment $\mathrm{pH}$ and Oxidation-Reduction Potential on Microbial Hydrocarbon Degradation," Applied and Environmental Microbiology, Vol 40, pp 365-369.

Hamoda, M. F., and Al-Haddad, A. A. 1988. "Treatment of Petroleum Refinery Effluents in a Fixed-Film Reactor," Water Science Technology, Vol 20.

Hance, R. J. 1967. "Relationship between Partition Data and the Adsorption of Some Herbicides by Soils," Nature, Vol 214, pp 630-631.

Hannah, S. A., Austern, B. M., Eralp, A. E., and Wise, R. H. 1986 (Jan). "Comparative Removal of Toxic Pollutants by Six Wastewater Treatment Processes," Journal Water Pollution Control Federation.

Hasan, S. M., and Hall, J. B. 1975. "The Physiological Function of Nitrate Reduction in Clostridium perfringens, " Journal of General Microbiology, Vol 87, pp 120-128.

Hassett, J. P., and Anderson, M. A. 1982. "Effects of Dissolved Organic Matter on Adsorption of Hydrophobic Organic Compounds by River - and SewageBorne Particles," Water Research, Vol 16, pp 681-686.

Healy, J. B., Young, L. J., and Reinhard, M. 1980. "Methanogenic Decomposition of Ferulic Acid, a Model Lignin Derivative," Applied and Environmental Microbiology, Vo1 39, pp 436-444.

Heitkamp, M. A., Franklin, W., and Cerniglia, C. E. 1988. "Microbial Metabolism of Polycyclic Aromatic Hydrocarbons: Isolation and Characterization of a Pyrene-Degrading Bacterium," Applied and Environmental Microbiology, Vol 54, pp 2549-2555.

Henson, J. M., Yates, M. V., Cochran, J. W., and Shackleford, D. L. 1988 . "Microbial Removal of Halogenated Methanes, Ethanes, and Ethylenes in an Aerobic Soil Exposed to Methane," FEMS Microbiology Ecology, Vol 53, pp 193-201.

Herbes, S. E. 1977. "Partitioning of Polycyclic Aromatic Hydrocarbons Between Dissolved and Particulate Phases in Natural Waters, "Water Research, Vol 11, pp 493-496.

Hickman, G. T., and Novak, J. T. 1989. "Relationship Between Subsurface Biodegradation Rates and Microbial Density," Environmental Science and Technology, Vol 23, No. 5, pp 525-532. 
Hickman, G. T., Novak, J. T., Morris, M. S., and Rebhun, M. 1989. "Effects of Site Variations on Subsurface Biodegradation Potential, " Journal of the Water Pollution Control Federation, Vol 61, No. 9, pp 1564-1575.

Hi11, D. O., Myers, T. E., and Brannon, J. M. 1988. "Development and Application of Techniques for Predicting Leachate Quality in Confined Disposal

Facilities: Background and Theory," Miscellaneous Paper D-88-1, US Army Engineer Waterways Experiment Station, Vicksburg, MS.

Hoffsommer, J. C., Kaplan, L. A., Glover, D. J., Kubose, D. A., Dickinson, C., Goya, H., Kayser, E. G., and Groves, C. L. 1978. "Biodegradability of TNT: A Three-Year Pilot Plant Study," Naval Surface Weapons Center, NSWC/WOL TR 77-136, Silver Springs, MD.

Horvath, R. S. 1972. "Microbial Co-metabolism and the Degradation of Organic Compounds in Nature," Bacteriological Reviews, Vo1 36, pp 146-155.

Huling, S. G. 1989. "Superfund Ground Water Issue," EPA/540/4-89/003, US Environmental Protection Agency, Robert S. Kerr Environmental Research Laboratory, Ada, OK.

Inoue, A., and Horikoshi, K. 1989. "A Pseudomonas Thrives in High Concentrations of Toluene," Nature, Vol 338, pp 264-266.

Irvine, R. L., Ketchum, L. H., Arora, M. L., and Barth, E. F. 1985 (Aug).

"An Organic Loading Study of Full-Scale Sequencing Batch Reactors," Journal Water Pollution Control Federation.

Jenkins, T. F., and Walsh, M. E. 1987. "Development of An Analytical Method for Explosive Residues in Soil," Report 87-7, US Army Engineer Cold Regions Research and Engineering Laboratory, Hanover, NH.

Jones, J. G. 1977. "The Long Term Effects of Kerosene Pollution on the Microflora of a Moorland Soil," Journal of Applied Bacteriology, Vol 43, pp $123-128$.

Jubboori, S. A., Stewart, G. L., and Adrian, D. D. 1974. "Aquifer Clogging in Combined Wastewater Recharge," Journal Water Pollution Control Federation, Vol 46, No. 13, pp 2732-2744.

Kampbe11, D. H., Wilson, J. T., Reed, H. W., and Stocksdale, T. T. 1987 (Oct) "Removal of Volatile Aliphatic Hydrocarbons in a Soil Bioreactor," International Journal of Air Pollution and Hazardous Waste Management, Vol 37 , No. 10 .

Kaplan, D. L., and Kaplan, A. M. 1982. "Thermophilic Biotransformations of 2,4,6-Trinitrotoluene under Simulated Composting Conditions, " Applied and Environmental Microbiology, Vol 44, pp 757-760.

Kaplan, D. L., Ross, E., Emerson, D., LeDoux, R., Mayer, J., and Kaplan, A. M. 1985. "Effects of Environmental Factors on the Transformation of 2,4, 6-Trinitrotoluene in Soils," Natick/TR-85/052, USANRDC, Natick, MA.

Kappeler, T., and Wuhrmann, K. 1978. "Microbial Degradation of the WaterSoluble Fraction of Gas 0il-II. Bioassays with Pure Strains, "Water Research, Vo1 12, pp 335-342.

Karickhoff, S. W. 1980. "Sorption Kinetics of Hydrophobic Pollutants in Natural Sediments," EPA/600J-80-105, Environmental Research Laboratory, Athens, GA. 
Karickhoff, S. W. 1981. "Semi-empirical Estimation of Sorption of Hydrophobic Pollutants on Natural Sediments and Soils," Chemosphere, Vol 10, pp 833-846.

Karickhoff, S. W., Brown, D. S., and Scott, T. A. 1979. "Sorption of Hydrophobic Pollutants on Natural Sediments," Water Res,, Vol 13, pp 241-248.

Kayal, S. I., and Connel1, D. W. 1990. "Partitioning of Unsubstituted Polycyclic Aromatic Hydrocarbons Between Surface Sediments and the Water Column in the Brisbane River Estuary," Australian Journal of Marine and Freshwater Research, Vo1 41, pp 443-456.

Keck, J., Sims, R. C., Coover, M., Park, K., and Symons, B. 1989. "Evidence for Cooxidation of Polynuclear Aromatic Hydrocarbons in Soil, "Water Research, Vol 21, No. 12, pp 1467-1476.

Keely J. F. 1989 (Oct). "Performance Evaluations of Pump-and-Treat Remediations," Ground Water Issue.

Kelly, H. G. 1987. "Pilot Testing for Combined Treatment of Leachate from a Domestic Waste Landfill Site," Journal WPCF, Vol 59, No. 5.

Kim, B. R., Chian, E. S., Cross, W. H., and Cheng, S. S. 1986 (Jan).

"Adsorption, Desorption, and Bioregeneration in an Anaerobic, Granular Activated Carbon Reactor for the Removal of Phenol," Journal Water Pollution Control Federation.

Kim, J. K., and Maier, W. J. 1986 (Feb). "Acclimation and Biodegradation of Chlorinated Organic Compounds in the Presence of Alternate Substrates," Journal Water Pollution Control Federation.

Kim, S. H., and Pirbazari, M. 1989. "Bioactive Adsorber Model for Industrial Wastewater Treatment," Journal of Environmental Engineer, ASCE, Vol 115, No. $6, \mathrm{p} 1235$.

Klecka, G. 1982. "Fate and Effects of Methylene Chloride in Activated Sludge," Applied and Environmental Microbiology, Vol 44, pp 701-707.

Knowles, R. 1979. "Denitrification and Ammonia Formation in Anaerobic Coastal Sediments," Applied and Environmental Microbiology, Vol 38, pp 486-493.

Koike, I., and Hattori, A. 1978. "Denitrification and Ammonia Formation in Anaerobic Coastal Sediments," Applied and Environmental Microbiology, Vol 35, pp 278-282.

Kosky, K. F., and Neff, C. R. 1990. "Innovative Biological Degradation System for Hydrocarbon treatment," Biofiltration Brochure.

Kuhn, E. P., Colberg, P. J., Schnoor, J. L., Wanner, 0., Zehnder, A. J. B., and Schwarzenbach, R. P. 1985. "Microbial Transformations of Substituted Benzenes During Infiltration of River Water to Groundwater: Laboratory Column Studies," Environmental Science and Technology, Vol 19, pp 961-968.

Kuhn, E. P., Suflita, J. M., Rivera, M. D., and Young, L. Y. 1989. "Influence of Alternate Electron Acceptors on the Metabolic Fate of Hydroxybenzoate Isomers in Anoxic Aquifer Slurries," Applied and Environmental Microbiology, Vol 55, pp 590-598. 
Landrum, P. F., Nihart, S. R., Eadie, B. J., and Gardner, W. S. 1984.

"Reverse-Phase Separation Method for Determining Pollutant Binding to Aldrich Humic Acid and Dissolved Organic Carbon of Natural Waters," Environmental Science and Technology, Vo1 18, pp 187-192.

LaPat-Polasko, L., McCarty, P. L., and Zehnder, A. J. B. 1984. "Secondary Substrate Utilization of Methylene Chloride by an Isolated Strain of Pseudomonas sp," Applied and Environmental Microbiology, Vol 47, pp 825-830.

Lappin-Scott, H. M., Cusack, F., and Costerton, J. W. 1988. "Nutrient Resuscitation and Growth of Starved Cells in Sandstone Cores: A Novel Approach to Enhanced Oil Recovery," Applied and Environmental Microbiology, Vol 54, pp 1373-1382.

Larson, R. J. 1984. "Kinetic and Ecological Approaches for Predicting Biodegradation Rates of Xenobiotic Organic Chemicals in Natural Ecosystems," in $M$. J. Klug and C. A. Reddy (Eds), Current Perspectives in Microbial Ecology, American Society for Microbiology, Washington, DC, pp 677-686.

Leggett, D. C., Jenkins, T. F., and Murrmann, R. P. 1977. "Composition of Vapors Evolved from Military TNT as Influenced by Temperature, Solid Composition Age, and Source," Special Report 77-16, US Army Engineer Cold Regions Research and Engineering Laboratory, Hanover, NH.

Lewis, D. L., Kolig, H. P., and Hodson, R. E. 1986. "Nutrient Limitation and Adaptation of Microbial Populations to Chemical Transformations." Applied and Environmental Microbiology, Vo1 51, pp 598-603.

Li, C. T., et. al. 1986. "Chemical Bio-Fluidized Bed Treatment of Slaughterhouse Wastewater," Journal of Environmental Engineering Division, American Society of Civil Engineers, Vol 4, p 718.

Li, W., Yin, P., and Yang, Y. 1987. "Properties of TNT-Degrading Enzymes in Intact Cells of Citrobacter freundi," ACTA Microbial Sinica, Vol 27, No. 3, pp 257-263.

Linton, J. D., and Drozd, J. W. 1982. "Microbial Interactions and Communities in Biotechnology," in A. T. Bull and J. H. Slater (Eds), Microbial Interactions and Communities, Vol 1, Academic Press, London, pp 357-406.

Lith, C. V. 1989. "Design Criteria for Biofilters," Air and Waste Management Association, 82nd Meeting and Exhibition.

Little, C. D., Palumbo, A. V., Herbes, S. E., Lidstrom, M. E., Tyndal1, R. L., and Gilmer, P. J. 1988. "Trichloroethylene Biodegradation by a MethaneOxidizing Bacterium," Applied and Environmental Microbiology, Vol 54, pp 951-956.

Liu, K. T., and Weber, W. J. 1981. "Characterization of Mass Transfer Parameters for Adsorber Modeling and Design," Journal of the Water Pollution Control Federation, Vo1 53, p 1541.

Lovely, D. R., Dwyer, D. F., and Klug, M. J. 1982. "Kinetic Analysis of Competition Between Sulfate Reducers and Methanogens for Hydrogen in Sediments," Applied and Environmental Microbiology, Vol 43, pp 1373-1379.

Lovely, D. R., and Klug, M. J. 1983. "Sulfate Reducers Can Outcompete Methanogens at Freshwater Sulfate Concentrations," Applied and Environmental Microbiology, Vol 45, pp 187-192. 
Lovely, D. R., and Lonergan, D. J. 1990. "Anaerobic Oxidation of Toluene, Phenol, and $p$-Cresol by the Dissimilatory Iron-Reducing Organism, GS-15." Applied and Environmental Microbiology, Vol 56, pp 1858-1864.

Loveley, D. R., and Phillips, E. J. 1986. "Availability of Ferric Iron for Microbial Reduction in Bottom Sediments of the Freshwater Tidal Potomac River," Applied and Environmental Microbiology, Vo1 52, pp 751-757.

Lowenbach, W. 1978. "Compilation and Evaluation of Leaching Test Methods," Contract Report D-75-4, US Army Engineer Waterways Experiment Station, Vicksburg, MS .

Lyman, W. J., Reehl, W. F., and Rosenblatt, D. H. 1990. Handbook of Chemical Property Estimation Methods, American Chemical Society, Washington, DC.

MacLeod, F. A., Lappin-Scott, H. M., and Costerton, J. W. 1988. "Plugging of a Model Rock System by Using Starved Bacteria," Applied and Environmental Microbiology, Vol 54, pp 1365-1372.

Major, D. W., Mayfield, C. I., and Barker, J. F. 1987. "Biotransformation of Benzene by Dentrification in Aquifer Sand," Ground Water, Vo1 26, pp 8-14.

Marrin, D. L., and Thompson, B. M. 1987. "Gaseous Behavior of TCE Overlying a Contaminated Aquifer," Ground Water, Vol 25, No. 1, pp 21-27.

Marsha11, K. C. 1971. "Sorption Interactions Between Soil Particles and Microorganisms," in A.D. McLaren and J. Skujins (Eds), Soil Biochemistry, Vol 2. Marcel Dekker, Inc., New York, pp 409-422.

1980. "Adsorption of Microorganisms to Soils and Sediments," in G. Bitton and K. C. Marshall (Eds), Adsorption of Microorganisms to Surfaces, John Wiley and Sons, Inc., New York, pp 317-329.

Martin, J. P., Haider, K., and Bondiett, E. 1972. "Properties of Model Humic Acids Synthesized by Phenoloxidase and Autooxidation of Phenols and Other Compounds Formed by Soil Fungi," in Proceedings of the International Meeting on Humic Substances, Wageningen. Belgium, pp 171-186.

McCa11, P. J., and Agin, G. L. 1985. "Desorption Kinetics of Picloram as Affected by Residence Time in the Soil, "Environmental Toxicology and Chemistry, Vol 4, p 37.

McCormick, N. G., Corne11, J. H., and Kaplan, A. M. 1978. "Identification of Biotransformation Products from 2,3-Dinitrotoluene," Applied and Environmental Microbiology, Vol 35, No 5, pp 945-948.

1981. "Biodegradation of Hexahydro-1,3,5-Trinitro-1,3,5-
Triazine," Applied and Environmental Microbiology, Vol 42, No. 5, pp 817-823.

McFarlane, J. C., Cross, A., Frank, C., and Rogers, R. D. 1981. "Atmospheric Benzene Depletion by Soil Microorganisms," Environmental Monitoring and Assessment, Vol 1, pp 75-81.

McKay, G., McKee, S., and Walters, H. R. J. 1987. "Solid-Liquid Adsorption Based on External Mass transfer, Macropore and Micropore Diffusion, " Chemical Engineering Science, Vo1 42, p 1145.

McShane, S. F., Lebel, A., Pollock, T. E., and Stirrat, B. A. 1986. "Biophysical Treatment of Landfill Leachate Containing Organic Compounds," 41st Purdue Industrial Waste Conference Proceedings, West Lafayette, IN. 
Mercer, J. W., Skipp, D. C., and Giffin, D. 1990. "Basics of Pump-And-Treat Ground-Water Remediation Technology," EPA/600/8-90-003, Robert S. Kerr Environmental Research Laboratory, US Environmental Protection Agency, Ada, OK.

Merck and Company. 1976. The Merck Index, 9th ed., Rahway, NJ .

Metcalf and Eddy, Inc. 1982. Wastewater Engineering, MacGraw Hill Inc.

Michelsen, D. L., Smith, J. W., and Suggs, J. A. 1988. "Use of Collidal Gas Aphrons for In-Situ Biodegradation of Contaminated Ground Water, "Proceedings of 14th Hazardous Waste Symposium, USEPA, Cincinnati, $\mathrm{OH}$.

Mihelcic, J. R., and Luthy, R. G. 1988. "Microbial Degradation of Acenaphtene and Naphtalene Under Denitrification Conditions in Soil-Water Systems," Applied and Environmental Microbiology, Vol 54, pp 1188-1198.

Milde, G., Nerger, F., and Merlger, R. 1988. "Biological Degration of Volatile Chlorinated Hydrocarbons in Grounwater, " Water Science Technology, Vo1 20, pp 67-73.

Miller, R. M., Singer, G. M., Rosen, J. D., and Bartha R. 1988. "Sequential Degradation of Chlorophenols by Photolytic and Microbial Treatment, "Environmental Science and Technology, Vol 22, No. 10.

Montemagno, C. D., and Irvine, R. L. 1989. "Feasibility of Biodegrading Trinitrotoluene (TNT) Contaminated Soils," Proceedings for the 14th Annual Army Environmental R \& D Symposium, US Army Toxic and Hazardous Materials Agency, Report No. CETHA-TE-TR-90055, pp 471-490.

Morgan, P., and Watkinson, R. J. 1989. "Microbiological Methods for the Cleanup of Soil and Ground Contaminated with Halogenated Organic Compounds." FEMS Microbiology Reviews, Vo1 63, pp 277-299.

1990. "Assessment of the Potential for In Situ Biotreatment of Hydrocarbon-Contaminated Soils," Water Science Technology, Vol 22, pp 63-68.

Morris, M. S., and Novak, J. T. 1989. "Mechanisms Responsible for the Biodegradation of Organic Compounds in the Subsurface," Journal of Hazardous Materials.

Murphy, E. M., Zachara, J. M., and Smith, S. C. 1990. "Influence of MineralBound Humic Substances on the Sorption of Hydrophobic Organic Compounds," Environmental Science and Technology, Vol 24, No. 10, pp 1507-1516.

Myers, T. E., and Brannon, J. M. 1988. "New Bedford Harbor Superfund Project, Acushnet River Estuary Engineering Feasibility Study of Dredging and Dredged Material Disposal Alternatives; Report 5, Evaluation of Leachate Quality," Technical Report EL-88-15, US Army Engineer Waterways Experiment Station, Vicksburg, MS.

Nathwani, J. S., and Phillips, C. R. 1979. "Rate Controlling Processes in the Release of Radium-226 From Uranium Mill Tailings," Water. Air, and Soil Pollution, Vol 11, pp 309-317.

National Academy of Sciences. 1975. "Fate of Petroleum in the Marine Environment," National Academy of Sciences, Washington, DC.

Naumova, R. P., Amerkhanova, N. N., and Shaykhutdinov, V. A. 1979. "Study of the First Stage of Trinitrotoluene Transformation by Psuedomanas denitrificans," Prikladnaya Biokhimiya i Mikrobiologiya, Vol 15, No. 1, pp 45-50.

Naumova, R. P., Selivanovskaya, S. Yu., and Cherepneva, I. E. 1988. "Trans formation of 2,4,6-Trinitrotoluene in Psuedomanas fluorescens in Case of Oxy- 
gen and Nitrate Respiration," Prikladnaya Biokhimiya i Mikrobiologiya, Vol 24, No. 4, pp 493-498.

Naumova, R. P., Selivanovskaya, S. Yu., and Mingatina, F. A. 1988. "The Possibility of 2,4,6-Trinitrotoluene Deep Destruction by Bacteria," Mikrobiologiya, Vol 57, No, 2, pp 218-222.

Neff, C. R. 1990. "Biofilter Technology for Odor Control," Biofiltration Inc. Brochure.

Nelson, D. W., and Sommers, L. E. 1982. "Total Carbon, Organic Carbon, and Organic Matter," Methods of Soil Analysis, Part 2 - Chemical and Microbiological Properties, 2nd ed., No. 9, American Society of Agronomy, Madison, WI.

Nelson, M. J. K., Montgomery, S. O., Mahaffey, W. R., and Pritchard, P. H. 1987. "Biodegradation of Trichloroethylene and Involvement of an Aromatic Biodegradative Pathway," Applied and Environmental Microbiology, Vo1 53, pp 949-954.

Ne1son, M. J. K., Montgomery, S. O., and Pritchard, P. H. 1988. "Trichloroethylene Metabolism by Microorganisms that Degrade Aromatic Compounds," Applied and Environmental Microbiology, Vol 54, No. 2, pp 604-606.

Nhung, M. F. M., and Ponnamperuma, F. N. 1966. "Effects of Calcium Carbonate, Manganese Dioxide, Ferric Hydroxide, and Prolonged Flooding on Chemical and Electrochemical Changes and Growth of Rice in a Flooded Acid Sulfate Soil," Soil Science, Vol 102, pp 29-41.

Nkedi-Kizza, P., Rao, P. S. C., and Hornsby, A. G. 1987. "Influence of Organic Cosolvents on Leaching of Hydrophobic Organic Chemicals Through Soils," Environmental Science and Technology, Vol 21, No. 11, pp 1107-1111.

Nkedi-Kizza, P., Rao, P. S. C., Jessup, R. E., and Davidson, J. M. 1982. "Ion Exchange and Diffusive Mass Transfer During Miscible Displacement Through an Aggregated Oxisol," Soil Science Society of America Journal, Vol 46, p 471.

Novak, J. T., Hickman, G. T., Morris, M. A., and Benoit, R. E. 1989. "An Investigation of the Biodegradation Potential of Groundwater Contaminants," Bulletin No. 165, Virginia Water Resources Research Center, Virginia Polytechnic Institute and State University, Blacksburg, VA.

0'Connor, D. J., and Connolly, J. P. 1980. "The Effect of Concentration of Adsorbing Solids on the Partition Coefficient," Water Research, Vol 14, pp 1517-1523.

Oliver, B. G. 1985. "Desorption of Chlorinated Hydrocarbons from Spiked and Anthropogenically Contaminated Sediments," Chemosphere, Vo1 14, p 1087.

Omori, T., and Yamada, K. 1979. "Studies on Utilization of Hydrocarbons by Microorganisms. Part XIII. Oxidation of $m$-Xylene and Pseudocumene by Pseudomonas aeruginosa," Agricultural Biology and Chemistry, Vol 33, No. 7, pp 979-985.

Opatken, E. J., et al. 1988. "Stringfellow Lechate Treatment with a RBC," Journal of Environmental Engineering Division, American Society of Civil Engineers, Vol 1, p. 41 .

Orgam, A. V., Jessup, R. E., Ou, L. T., and Rao, P. S. C. 1985. "Effects of Sorption on Biological Degradation Rates of (2,4-Dichlorophenoxy)acetic Acid in Soils," Applied and Environmental Microbiology, Vol 49, pp 582-587. 
Ostendorf, D. W. 1990. "Long Term Fate and Transport of Immiscible Aviation Gasoline in the Subsurface Environment, "Water Science Technology, Vol 22, No. 6 , pp $37-44$.

Ottengraf, S. P., and van den Oever, A. H. 1983. "Kinetics of Organic Compound Removal from Waste Gases with a Biological Filter," Biotechnology and Bioengineering, Vol XXV.

Palit, T., and Qasim, M. 1977. "Biological Treatment Kinetics of Landfill Leachate," Journal of Environmental Engineering Division, American Society of Civil Engineers, Vol 103, No. 2.

Parker, J. C., and Valocchi, A. J. 1986. "Constraints on the Validity of Equilibrium and First-Order Kinetic Transport Models in Structured Soils," Water Resources Research, Vol 23, pp 2187.

Pate1, R. N. 1984. "Methane Monooxygenase from Methylobacterium sp. Strain CRL-26," in R. L Crawford and R. S. Hanson (Eds), Microbial Growth on C1 Compounds, American Society for Microbiology, Washington, DC, pp 83-90.

Patrick, W. H., Jr. 1958. "Modification of Method of Particle Size Analysis," Proceedings, Soil Science Society of America, Vol 22, pp 366-367.

Patrick, W. H., Jr., and Mikkelsen, D. S. 1971. "Plant Nutrient Behavior in Flooded Soil," in Fertilizer Technology and Use, Soil Science Society of America, Madison, WI.

Payne, W. J. 1973. "Reduction of Nitrogenous Oxides by Microorganisms," Bacteriological Reviews, Vol 37, pp 409-452.

Peel, R., and Benedek, A. 1976. "The Modelling of Activated Carbon Adsorbers in the Presence of Biooxidation, "Water, AiCHE Series No. 166.

Pennington, J. C., and Patrick, W. H., Jr. 1990. "Adsorption and Desorption of 2,4,6-Trinitrotoluene by Soils," Journal of Environmental Quality, Vol 19, No. 3, pp 559-567.

Perry, J. J. 1984. "Microbial Metabolism of Cyclic Alkanes," in R. M. Atlas (Ed), Petroleum Microbiology, Macmillan Publishing Company, New York, pp 61-97.

Peters, R. W., and Alleman, J. E. 1982. "The History of Fixed-Film Wastewater Treatment Systems, " Proceedings of First International Conference on Fixed-Film Biological Processes, USAE-CERL, Champaign, IL.

Phelps, T. J., Ringelberg, D., Hedrick, D., Davis, J., Fliermans, C. B., and White, D. C. 1988. "Microbial Biomass and Activities Associated with Subsurface Environments Contaminated with Chlorinated Hydrocarbons," Geomicrobiology Journal, Vol 6, pp 157-170.

Pierce, G. E., Robinson, J. B., Garrett, G. E., and Sojka, S. A. 1984. "Cloning of the Chlorotoluene Gene," Developments in Industrial Microbiology, Vol 25, pp 597-602.

Ponnamperuma, F. N. 1972. "The Chemistry of Submerged Soils," Advances in Agronomy, Vol 24, pp 29-88.

Rao, P. S. C. 1990. "Sorption of Organic Contaminants," Water Science Technology, Vol 22, No. 6, pp 1-6. 
Rao, P. S. C., Rolston, D. E., Jessup, R. E., Davidson, J. M., and Kilcrease, D. P. 1980. "Experimental and Mathematical Description of Nonabsorbed Solute Transfer by Diffusion in Spherical Aggregates," Soil Science Society of America Journal, Vo1 44, p 1139.

Raymond, R. L., Jamison, V. W., and Hudson, J. O. 1976. "Beneficial Stimulation of Bacterial Activity in Groundwaters Containing Petroleum Products," Physical, Chemical Wastewater Treatment, AiCHe Symposium Series.

Reddy, K. R., and Patrick, W. H., Jr. 1975. "Effect of Alternate Aerobic and Anaerobic Conditions on Redox Potential, Organic Matter Decomposition, and Nitrogen Loss in a Flooded Soil." Soil Biology and Biochemistry, Vol 7 , pp 87-94.

Reddy, K. R., Rao, P. S. C., and Patrick, W. H., Jr. 1980. "Factors Influencing Oxygen Consumption Rates in Flooded Soils, " Journal of the Soil Science Society of America, Vo1 44, pp 741-744.

Remberger, M., Allard, A.-S., and Neilson, A. H. 1986. "Biotransformation of Chloroguaiacols, Chlorocatechols, and Chloroveratrols in Sediments, "Applied and Environmental Microbiology, Vol 51, pp 552-558.

Ridgeway, H. F., Phipps, D. W., Safarik, J., Haag, F., Reinhard, M. Ball, and McCarty, P. L. 1988. "Investigation of the Transport and Fate of Gasoline Hydrocarbon Pollutants in Groundwater, "Final Report Submitted by Orange County Water District to US Geological Survey, Reston, VA.

Rijnaarts, H. H. M. , Backmann, A., Jumelet, J. C., and Zehnder, A. J. B. 1990. "Effect of Desorption and Intraparticle Mass Transfer on the Aerobic Biomineralization of alpha-Hexachlorophene in a Contaminated Calcareous Soil," Environmental Science and Technology, Vol 24, No. 9, pp 1349-1354.

Riss, A., and Schwiesfurth, R. 1988. "Degradation of Hydrocarbons Using Nitrate as an Electron Acceptor-Selected Findings," Dechema Biotechnology Conferences.

Rittman, B. E. 1982. "Comparative Performance of Biofilm Teactor Types," Biotechnology and Bioengineering, Vol XXIV.

Roberts, P. V., Cornel, P., and Summers, R. S. 1985. "External Mass-Transfer Rate in Fixed-Bed Adsorption," Journal Environmental Engineering Division, American Society of Civil Engineers, Vol 111.

Roberts, P. V., Goltz, M. N., Summers, R. S., Crittenden, J. C., and NkediKizza, P. 1987. "The Influence of Mass Transfer on Solute Transport in Co1umn Experiments with an Aggregated Soil, " Journal of Contaminant Hydrology, Vo1 1, pp 375-393.

Robinson, K. G., Farmer, W. S., and Novak, J. T. 1990. "Availability of Sorbed Toluene in Soils for Biodegradation by Acclimated Bacteria," Water Research, Vol 24, pp 345-350.

Rosenberg, E., Zuckerberg, A., Rubinovitz, C., and Gutnik, D. L. 1979. "Emulsifier of Arthrobacter RAG-1: Isolation and Emulsifying Properties," Applied and Environmental Microbiology, Vol 37, pp 403-408.

Roszak, D. B., and Colwell, R. R. 1987. "Survival Strategies of Bacteria in the Natural Environment," Microbiological Reviews, Vo1 51, No. 3, pp 365-379.

Rozich, A. F., and Gaudy, A. F. 1985 (Jul). "Response of Phenol Activated Sludge Process to Quantitative Shock Loadings," Journal Water Pollution Control Federation. 
Rubin, H. E., and Alexander, M. 1983. "Effect of Nutrients on the Rates of Mineralization of Trace Concentrations of Phenol and $p$-Nitrophenol, "Environmental Science and Technology, Vol 17, pp 104-107.

Russel1, J. D., Cruz, M., White, J. L., Bailey, G. W., Payne, W. R., Pope, J. D., and Teasley, J. I. 1968. "Mode of Chemical Degradation of s-Triazines by Montmorillonite," Science, Vol 160, pp 1340-1342, cited in Orgam, A. V., Jessup, R. E., and Rao, P. S. C. 1985. "Effects of Sorption on Biological Degradation Rates of (2,4-Dichlorophenoxy)acetic Acid in Soils, "Applied and Environmental Microbiology, Vol 49, pp 582-587.

Sanford, R., and Smallbeck, D. 1987. "The Enrichment and Isolation of a Ketone Degrading Microbial Consortium by Continuous Culture Techniques to Model Contaminated Groundwater Treatment," Abstracts from the 1987 Annual Meeting of the American Society of Microbiology.

Schmidt, S. K., and Alexander, M. 1985. "Effects of Dissolved Organic Carbon and Second Substrates on the Biodegradation of Organic Compounds at Low Concentrations," Applied and Environmental Microbiology, Vol 49, pp 822-827.

Schollenberger, L. J., and Simon, R. H. 1945. "Determination of Exchange Capacity and Exchangeable Bases in Soil-Ammonium Acetate Method," Soil Science, Vol 9, pp 13-25.

Schultz, J. R., and Keinath, T. M. 1984. "Powdered Activated Carbon Treatment Process Mechanisms, " Journal Water Pollution Control Federation, Vol 56, No. 2, p 143

Schwarzenbach, R. P., and Westall, J. 1981. "Transport of Nonpolar Organic Compounds from Surface Water to Groundwater. Laboratory Sorption Studies," Environmental Science and Technology, Vol 15, pp 1360-1367.

Scott, H. D., Wolf, D. C., and Lavy, T. L. 1982. "Apparent Adsorption and Microbial Degradation of Phenol by Soil, " Journal of Environmental Quality, Vol 11, No. 1, pp 107-112.

Scow, K. M., Schmidt, S. K., and Alexander, M. 1989. "Kinetics of Biodegradation of Mixtures of Substrates in Soil, " Soil Biology and Biochemistry, Vol 21, No. 5, pp 703-708.

Sebba, F. 1971. Journal Colloid International Science, Vo1 35, p. 643. Sebba, F., and Bennett, S. M. 1981. Proceedings of the Second International Congress on Chemical Engineering, Vol 4, p. 27.

Selivanovskaya, S. Yu., Akhmetova, D. Z., Naumova, R. P., and Ulyanov-Lenin, V. I. 1986. "Terminal Steps in the Preparatory Metabolism of 2,4,6-Trinitrotoluene in Pseudomonas fluorescens," Mikrobiologiya, Vol 55, No. 6, pp 1040-1041.

Sikka, H. C., Banerjee, S., Pack, E. J., and Appleton, H. T. 1980. "Environmental Fate of RDX and TNT," Final Report Contract No. DAMD17-77-C-7026, Syracuse Research Corporation, Syracuse, NY, for US Army Medical Research and Development Command, Fort Detrick, Frederick, MD.

Singer, M. E., and Finnerty, W. R. 1984. "Microbial Metabolism of StraightChain and Branched Alkanes," in R. M. Atlas (Ed), Petroleum Microbiology, Macmillan Publishing Company, New York, pp 1-59.

Slater, J. H. 1979. "Microbial Population and Community Dynamics," in J. M. Lynch and N. J. Poole (Eds), Microbial Ecology: A Conceptual Approach, John Wiley and Sons, New York, pp 45-63. 
Smith, B. E., and Sykes, J. F. 1988. "Recovery of DNAPL-Theory and Practice", Hazardous Wastes and Hazardous Materials.

Sokatch, J. R. 1969. Bacterial Physiology and Metabolism, Academic Press, New York.

Song, H.-G., and.Bartha, R. 1990. "Effects of Jet Fuel Spills on the Microbial Community of Soil," Applied and Environmental Microbiology, Vol 56, pp 646-651.

Song, H.-G., Wang, X., and Bartha, R. 1990. "Bioremediation of Terrestrial Fuel Spills," Applied and Environmental MIcrobiology, Vol 56, pp 652-656.

Sorensen, J. 1978. "Capacity for Denitrification and Reduction of Nitrate to Ammonia in a Coastal Marine Sediment," Applied and Environmental Microbiology, Vo1 35, pp 301-305.

Spain, J. C., Pritchard, P. H., and Bourquin, A. W. 1980. "Effects of Adaptation on Biodegradation Rates in Sediment/Water Cores from Estuarine and Freshwater Environments," Applied and Environmental Microbiology, Vol 40, pp $726-734$.

Spain, J. C., and Van Veld, P. A. 1983. "Adaptation of Natural Microbial Communities to Degradation of Xenobiotic Compounds: Effects of Concentration, Exposure, Time, Inoculum, and Chemical Structure," Applied and Environmental Microbiology, Vo1 45, pp 428-435.

Spanggord, R. J., Mill, T., Chou, T. - W., Mabey, W. R., Smith, J. H., and Lee, S. 1980. "Environmental Fate Studies on Certain Munition Wastewater Constituents: Final Report, Phase II-Laboratory Studies," AD82372, US Army Medical Research and Development Command, Fort Detrick, Frederick, MD.

Speitel, G. E. et al. 1989. "Biodegradation of Trace Concentration of Substituted Phenols in Granular Activated Carbon Columns," Environmental Science and Technology, Vol 23, No. 1, p 68.

Speitel, G. E. et al. 1990. "Mathematical Modelling of Bioregeneration in GAC Columns," Journal of Environmental Engineering, ASCE, Vol 116, No. 1, p. 32 .

Sper1, G. T., and McKae, J. 1980. "Microbial Growth on 2-Bromobutane," Antonie van Leeuwenhoek, Vo1 46, pp 331-341.

Subba-Rao, R. V., and Alexander, M. 1982. "Effect of Sorption on Mineralization of Low Concentrations of Aromatic Compounds in Lake Water Samples," Applied and Environmental Microbiology, Vol 44, pp 659-668.

Suflita, J. M., Gibson, S. A., and Beeman, R. E. 1988. "Anaerobic Biotransformations of Pollutant Chemicals in Aquifers," Journal of Industrial Microbiology, Vol 3, pp 179-194.

Suflita, J. M., Horowitz, A., Shelton, D. R., and Tiedje, J. M. 1982. "Dehalogenation: A Novel Pathway for Anaerobic Biodegradation of Haloaromatic Compounds," Science, Vol 218, pp 1115-1117.

Swindoll, C. M., Aelion, C. M., and Pfaender, F. K. 1988 (Jan). "Influence of Inorganic and Organic Nutrients on Aerobic Biodegradation and on the Adaptation Response of Subsurface Microbial Communities," Applied and Environmental Microbiology. 
Tabak, H. H., Quave, S. A., Mashni, C. I., and Barth, E. F. 1981. "Biodegradability Studies with Organic Priority Pollutant Compounds," Journal of the Water Pollution Control Federation, Vol 53, pp 1503-1518.

Takai, Y., and Kamura, T. 1966. "The Mechanism of Reduction in Waterlogged Paddy Soil," Folia Microbiologia, Vol 11, pp 304-313.

Tesezo, M., and Benedek, A. 1980. "Removal of Organic Substrates by Biologically Activated Carbon in a Fluidized Bed Reactor," Journal Water Pollution Control Federation, Vo1 52, No. 3, p 578.

Thibodeaux, L. J. 1979. Chemodynamics, John Wiley and Sons, New York.

Thiele, J., Muller, R., and Lingens, K. 1987. "Initial Characterization of a 4-Chlorobenzoate Dehalogenase from Pseudomonas sp. CBS3." FEMS Microbiological Letters, Vol 41, pp 115-119.

Thimann, K. V. 1963. The Life of Bacteria, The Macmillan Company, New York.

Thomas, J. M., Gordy, V. R., Fiorenza, S., and Ward, C. H. 1990. "Biodegradation of BTEX in Subsurface Materials Contaminated with Gasoline: Granger, Indiana," Water Science Technology, Vol 22, pp 53-62.

Thomas, J. M., and Ward, C. H. 1989. "In Situ Biorestoration of Organic Contaminants in the Subsurface," Environmental Science and Technology, Vol 23, No. 7 .

Travis, C. C., and Doty, C. B. 1990. "Can Contaminated Aquifers and Superfund Sites Be Remediated," Environmental Science and Technology, Vol 24, No. 10, pp 1464-1466.

Trudgill, P. W. 1984. "Microbial Degradation of the Alicyclic Ring," in D. T. Gibson (Ed), Microbial Degradation of Organic Compounds, Marcel Dekker, Inc., New York, pp 131-180.

Unkefer, P. J., Hanners, J. L., Unkefer, C. J., and Krammer, J. F. 1989. "Microbial Culturing for Explosives Degradation," Proceedings for the 14th Annual Army Environmental R \& D Symposium, US Army Toxic and Hazardous Materials Agency, Report No. CETHA-TE-TR-90055, p 151.

Updegraff, D. M. 1983. "The Effect of Microorganisms on the Permeability and Porosity of Petroleum Reservoir Rock," in J. E. Zajick, D. G. Cooper, T. R. Jack, and N. Kosaric (Eds), Microbial Enhanced Oil Recovery, PennWell Publishing Company, Tulsa, OK, pp 37-44.

Urbanski, T. 1964. Chemistry and Technology of Explosives, Vol. 1, The Macmillan Co., New York.

US Environmental Protection Agency. 1986. Test Methods for Evaluating Solid Waste: 5W846, USEPA Report No. 5W846, Washington, DC.

Valocchi, A. J. 1985. "Validity of the Local Equilibrium Assumption for Modeling Sorbing Solute Transport through Homogeneous Soils," Water Resources Research, Vol 21, p 808 .

- 1988. "Theoretical Analysis of Deviations from Local Equilibrium during Sorbing Solute Transport through Idealized Stratified Aquifers," Journal of Contaminant Hydrology, Vol 2, p 191.

van der Zee, S. E., van Riemsdijk, W. H., and de Haan, F. A. 1985 (Nov).

"Reaction Kinetics and Transport of Phosphate Assessment and Modeling," Contaminated Soil, First International TNO Conference on Contaminated Soil. 
Venkataramani, E. S., and Ahlert, R. C. 1984. "Rapid Aerobic Biostabilization of High Strength Industrial Landfill Leachate," Journal WPCF, 56, 11.

Verschueren, K., ed. 1983. Handbook of Environmental Data on Organic Chemicals, Van Nostrand Reinhold Company, New York.

Voge1, T. M., and McCarty, P. L. 1985. "Biotransformation of Tetrachloroethylene to Trichloroethylene, Dichloroethylene, Vinyl Chloride, and Carbon Dioxide Under Methanogenic Conditions," Applied and Environmental Microbiology, Vol 49, pp 1080-1083.

1987. "Abiotic and Biotic Transformations of 1,1

1-Trichloroethylene Under Methanogenic Conditions," Environmental Science and Technology, Vol 21, pp 1208-1213.

Voice, T. C., Rice, C. P., and Weber, W. J., Jr. 1983. "Effect of Solids Concentration on the Sorption Partitioning of Hydrophobic Pollutants in Aquatic Systems," Environmental Science and Technology, Vol 17, pp 513-518.

Wackett, L. P., and Gibson, D. T. 1988. "Degradation of Trichloroethylene by Toluene Dioxygenase in Whole-Cel1 Studies with Pseudomonas putida F1, " Applied and Environmental Microbiology, Vo1 54, pp 1703-1708.

Walker, J. D., and Colwell, R. R. 1974. "Microbial Degradation of Mode1 Petroleum at Low Temperature," Microbial Ecology, Vol 1, pp 63-95.

Wang, Y. T., Pai, P. C., and Latchaw, J. L. 1989 (Apr). "Effects of Preozonation on Anaerobic Biodegradability of 0-Cresol," Journal of Environmental Engineering, ASCE.

Ward, D. M., and Brock, T. D. 1978. "Anaerobic Metabolism of Hexadecane in Marine Sediments," Geomicrobiology Journal, Vol 1, pp 1-9.

Weber, W. J., Jr. 1972. Physicochemical Processes for Water Quality Control, Wiley-Interscience, New York.

Westlake, D. W. S., Jobson, A. M., and Cook, F. D. 1978. "In Situ Degradation of Oil in a Soil of the Boreal Region of the Northwest Territories." Canadian Journal of Microbiology, Vol 24, pp 254-260.

Wilson, B. H., Smith, G. B., and Rees, J. F. 1986. "Biotransformations of Selected Alkylbenzenes and Halogenated Aliphatic Hydrocarbons in Methanogenic Aquifer Material," Environmental Science and Technology, Vol 20, pp 997-1002.

Wilson, J. T., Henson, J. M., Piwoni, M. D., Wilson, B. H., and Banerjee, P. 1988. "Biodegradation and Sorption of Organic Solvents and Hydrocarbon Fuel Constituents in Subsurface Environments," Technical Report ESL-TR-87-52, US Environmental Protection Agency, Robert S. Kerr Environmental Research Laboratory, Ada, OK.

Wilson, J. T., McNabb, Cochran, J. W., Wang, T. H., Tomson, M. B., and Bedient, P. B. 1985. "Influence of Microbial Adaptation on the Fate of Organic Pollutants in Ground Water, "Environmental Toxicology and Chemistry, Vo1 4, pp 721-726.

Wilson, J. T., and Wilson, B. H. 1985. "Biotransformation of Trichloroethylene in Soil," Applied and Environmental Microbiology, Vol 49, pp 242-243.

Winfrey, M. R., and Ward, D. M. 1983. "Substrates for Sulfate Reduction and Methane Production in Intertidal Sediments, "Applied and Environmental Microbiology, Vo1 45, pp 193-199. 
Winfrey, M. R., and Zeikus, J. G. 1977. "Effect of Sulfate on Carbon and Electron Flow during Microbial Methanogenesis in Freshwater Sediments," Applied and Environmental Microbiology, Vol 33, pp 275-281.

Yoshida, T. 1975. "Microbial Metabolism of Flooded Soils," in E. A. Paul and A. D. McLaren (eds) Soil Biochemistry Vo1 1, Marcel Dekker, Inc., New York.

Young, L. Y. 1984. "Anaerobic Degradation of Aromatic Compounds," in D. T. Gibson (ed), Microbial Degradation of Organic Compounds, Marcel Dekker, Inc., New York, p 487-523.

Zaidi, B. R., Murakami, Y., and Alexander, M. 1988. "Factors Limiting Success of Inoculation to Enhance Biodegradation at Low Concentrations of Organic Chemicals," Environmental Science and Technology, Vol 22, pp 1419-1425.

Zaidi, B. R., Stucki, G., and Alexander, M. 1988. "Low Chemical Concentration and $\mathrm{pH}$ as Factors Limiting the Success of Inoculation to Enhance Biodegradation," Environmental Toxicology and Chemistry, Vol 7, pp 143-151.

Zajick, J. E., and Mahomedy, A. Y. 1984. "Biosurfactants: Intermediates in the Biosynthesis of Amphipathic Molecules in Microbes," in R. M. Atlas (ed), Petroleum Microbiology, Macmillan Publishing Company, New York, pp 221-297.

Zappi, M. E., Adrian, D. D., and Francingues, N. R. 1990. "Reduction of Effluent Recharge Capacity at The North Boundary Treatment System, Rocky Mountain Arsenal, Commerce City, Colorado," 1990 HMCRI Hazardous Wastes and Hazardous Materials Conference, HMCRI.

Zappi, M. E., Shafer, R. A., and Adrian, D. D. 1990. Compatability of Ninth Avenue Site Groundwater with Two Soil-Bentonite Slurry Wall Backfill Mixtures, Report No. MP-EL-90-9, US Army Engineer Waterways Experiment Station, Vicksburg, MS .

Zappi, M. E., Teeter, C. L., and Francingues, N. R. 1990. "Treatability of Contaminated Groundwater using Biological Processes," 11th Annual Superfund 1990 Conference, HMCRI.

- 1991. Treatability of Ninth Avenue Superfund Site Groundwater,

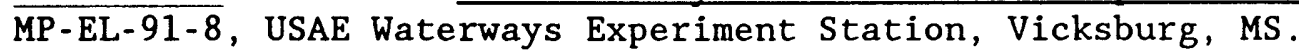

Zeyer, J., Kuhn, E. P., and Schwarzenbach, R. P. 1986. "Rapid Microbial Mineralization of Toluene and 1,3-Dimethylbenzene in the Absence of Molecular Oxygen, Applied and Environmental Microbiology, Vol 54, pp 490-496

Zimpro Inc., Meida1, John A., and Peterson, R. L. 1990. "The Treatment of Contaminated Groundwater and RCRA Wastewater at Bofors-Nobel, Inc., " Zimpro Inc. Technical Report No. HT-601, Presented at the 4th Annual RCRA Conference on Hazardous Waste and Hazardous Materials (HMCRI). 
Table 1

\section{Analytical Methods}

Organic Compounds

\begin{tabular}{|c|c|c|c|}
\hline Parameter & Matrix & Method* & Instrument \\
\hline $\begin{array}{l}\text { Methyl ethyl ketone } \\
\text { (MEK) }\end{array}$ & $\begin{array}{l}\text { Liquid } \\
\text { Soil } \\
\text { Vapor }\end{array}$ & $\begin{array}{lll}\text { EPA } & \text { SW } & 846-8260 \\
\text { EPA } & \text { SW } & 846-8260 \\
\text { EPA } & 600 / 4-84-041\end{array}$ & $\begin{array}{l}\text { MS /GC } \\
\text { MS/GC } \\
\text { MS/GC }\end{array}$ \\
\hline $\begin{array}{l}\text { Benzene, ethylbenzene } \\
\text { toluene, xylene } \\
\text { (BETX) }\end{array}$ & $\begin{array}{l}\text { Liquid } \\
\text { Soil } \\
\text { Vapor }\end{array}$ & $\begin{array}{lll}\text { EPA } & \text { SW } & 846-8260 \\
\text { EPA } & \text { SW } 846-8260 \\
\text { EPA } & 600 / 4-84-041\end{array}$ & $\begin{array}{l}\text { MS /GC } \\
\text { MS/GC } \\
\text { MS /GC }\end{array}$ \\
\hline $\begin{array}{l}\text { Tricholorethene } \\
\text { (TCE) }\end{array}$ & $\begin{array}{l}\text { Liquid } \\
\text { Soil } \\
\text { Vapor }\end{array}$ & $\begin{array}{lll}\text { EPA } & \text { SW } 846-8260 \\
\text { EPA } & \text { SW } 846-8260 \\
\text { EPA } & 600 / 4-84-041\end{array}$ & $\begin{array}{l}\text { MS /GC } \\
\text { MS/GC } \\
\text { MS/GC }\end{array}$ \\
\hline $\begin{array}{l}2,4,6 \text {-trinitrotoluene } \\
\quad(\text { TNT) }\end{array}$ & $\begin{array}{l}\text { Liquid } \\
\text { Soil } \\
\text { Vapor }\end{array}$ & $\begin{array}{lll}\text { EPA } & \text { SW } & 846-8330 \\
\text { EPA } & \text { SW } 846-8330 \\
\text { EPA } & 600 / 4-84-041\end{array}$ & $\begin{array}{l}\text { HPLC } \\
\text { HPLC } \\
\text { MS/GC }\end{array}$ \\
\hline $\begin{array}{l}\text { Hexahydro }-1,3,5- \\
\text { trinitro-1, 3, 5- } \\
\text { triazine (RDX) }\end{array}$ & $\begin{array}{l}\text { Liquid } \\
\text { Soil } \\
\text { Vapor }\end{array}$ & $\begin{array}{lll}\text { EPA } & \text { SW } 846-8330 \\
\text { EPA } & \text { SW } 846-8330 \\
\text { EPA } & 600 / 4-84-041\end{array}$ & $\begin{array}{l}\text { HPLC } \\
\text { HPLC } \\
\text { MS /GC }\end{array}$ \\
\hline $\begin{array}{l}\text { Polynuclear Aromatic } \\
\text { Hydrocarbons } \\
\text { (PAH's) }\end{array}$ & $\begin{array}{l}\text { Liquid } \\
\text { Soil } \\
\text { Vapor }\end{array}$ & $\begin{array}{lll}\text { EPA } & \text { SW } & 846-3510 \\
\text { EQP } & \text { SW } & 846-8270 \\
\text { EPA } & \text { SW } & 846-3540 \\
\text { EPA } & \text { SW } & 846-8270 \\
\text { EPA } & 600 / 4-84-041\end{array}$ & $\begin{array}{l}\text { MS /GC } \\
\text { MS /GC } \\
\text { MS /GC }\end{array}$ \\
\hline BNA's & $\begin{array}{l}\text { Water } \\
\text { Water and } \\
\text { Solid Extr. }\end{array}$ & $\begin{array}{ll}\text { Fed } & \text { Reg } 1984625 \\
\text { EPA } & \text { SW } 846-8270\end{array}$ & MS /GC \\
\hline
\end{tabular}

MS/GC - Mass spec/gas chromatography, Hewlett-Packard Model 5996.

HPLC - High Performance Liquid Chromatography, Waters 911-MS.

* EPA SW-846 - USEPA, Test Methods for Evaluating Solid Waste, SW 846. Third Edition, November 1986 with December 1988 revisions Office of Solid Waste and Emergency Response. Washington, DC 20460. 
Table 2

Analytical Methods

Gross Pollutants

\begin{tabular}{|c|c|c|c|}
\hline Parameter & Matrix & Methodx & Instrument \\
\hline $\begin{array}{c}\text { Chemical Oxygen } \\
\text { Demand (COD) }\end{array}$ & Water & $\begin{array}{l}\text { EPA } 600-4-79-020 \\
410.4\end{array}$ & $\begin{array}{l}\text { Technicon } \\
\text { AutoAnalyzer }\end{array}$ \\
\hline $\begin{array}{l}\text { Biochemical Oxygen } \\
\text { Demand (BOD) }\end{array}$ & Water & Std Methods 507 & $\begin{array}{ll}\text { YSI Probe } & 5720 \\
\text { YSI Model } 57\end{array}$ \\
\hline $\begin{array}{l}\text { Suspended Solids } \\
\text { (SS) }\end{array}$ & Water & Std Methods 209C & $\begin{array}{l}\text { VWR Scientific } \\
\text { Oven }\end{array}$ \\
\hline $\begin{array}{l}\text { Total Organic } \\
\text { Carbon (TOC) }\end{array}$ & $\begin{array}{l}\text { Soil/ } \\
\text { Water }\end{array}$ & SW $846-9060$ & $\begin{array}{l}\text { Total Organic } \\
\text { Carbon Analyzer }\end{array}$ \\
\hline $\begin{array}{l}\text { Total Recoverable } \\
\text { Petroleum Hydro- } \\
\text { carbons (TRPH) }\end{array}$ & $\begin{array}{l}\text { Soil/ } \\
\text { Water }\end{array}$ & EPA $600 / 4-79-020-418.1$ & IR \\
\hline
\end{tabular}

* Std Methods - American Public Health Association, Standard Methods for Examination of Water and Wastewater, 16th Edition, 1985. 1015 Fifteenth Street NW, Washington, DC 20005. EPA SW-846 - USEPA, Test Methods for Evaluating Solid Waste, SW 846. Third Edition, November 1986 with December 1988 revisions. Office of Solid.Waste and Emergency Response. Washington, DC 20460. EPA 600-4-79-020 - EPA Methods for Water and Waste. 
Table 3

Analytical Methods

Metals

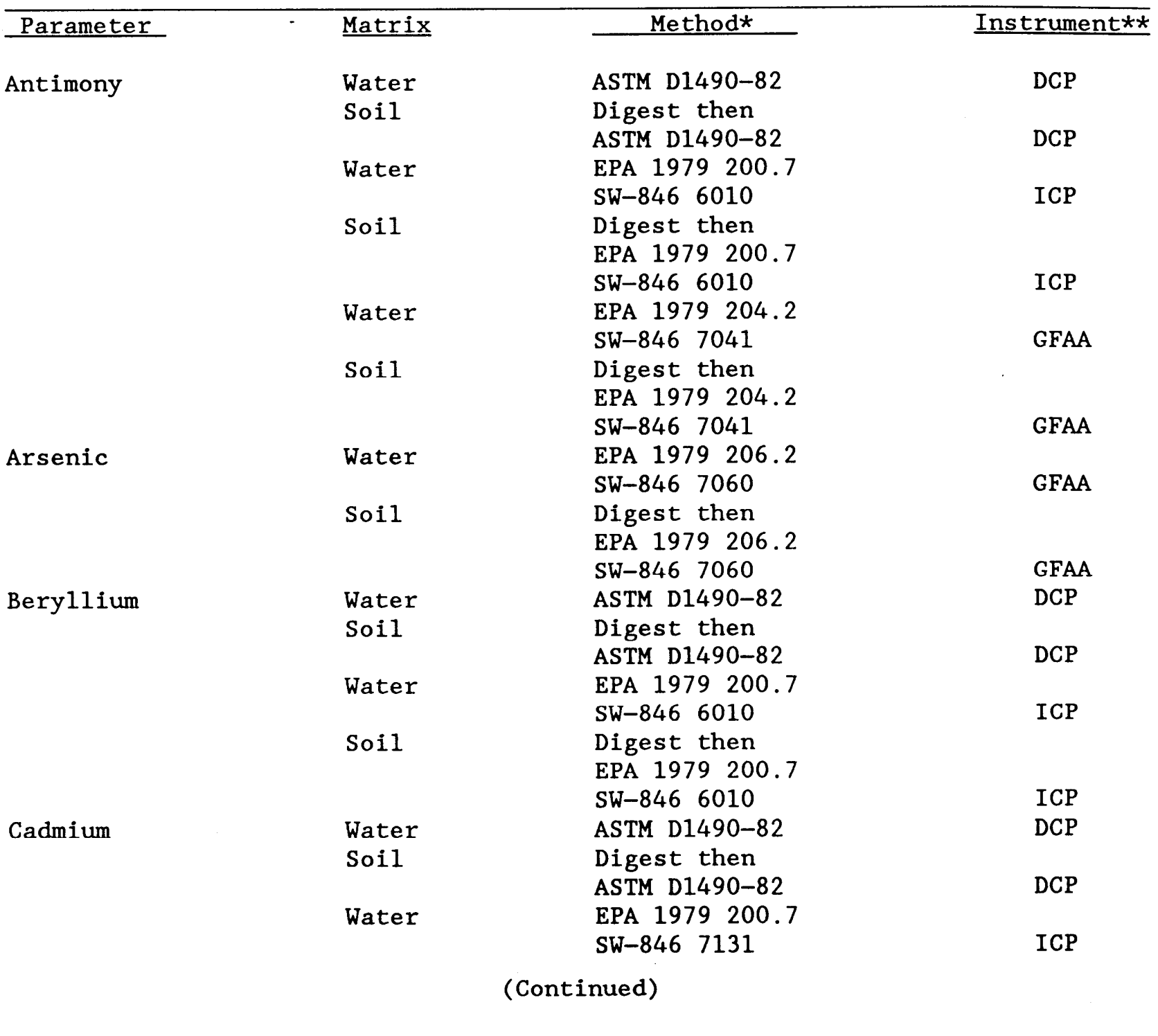

* ASTM - 19187 Annual Book of ASTM Standards, Water and Environmental Technology Vo1 11.01 and 11.02. 1987. 1916 Race Street, Philadelphia, PA 19103. EPA 1979 - USEPA, Methods of Chemical Analysis of Water and Wastes, EPA 600/4-79-020, March 1979 and EPA 600/4-82-055 Dec 1982. Environmental Monitoring and Support. Laboratory, Cincinnati, OH 45268. SW-846 - USEPA, Test Methods for Evaluating Solid Waste, SW-846 Third Edition, November 1986 with December 1988 revisions. Office of Solid Waste and Emergency Response, Washington, DC 20460.

** DCP - Direct Current Argon Emission Spectrometer; ICP - Inductively Coupled Argon Plasma Emission Spectrometer; GFAA - Graphite Furnace Atomic Adsorption; AA - Atomic Adsorption. 
Table 3 (Continued)

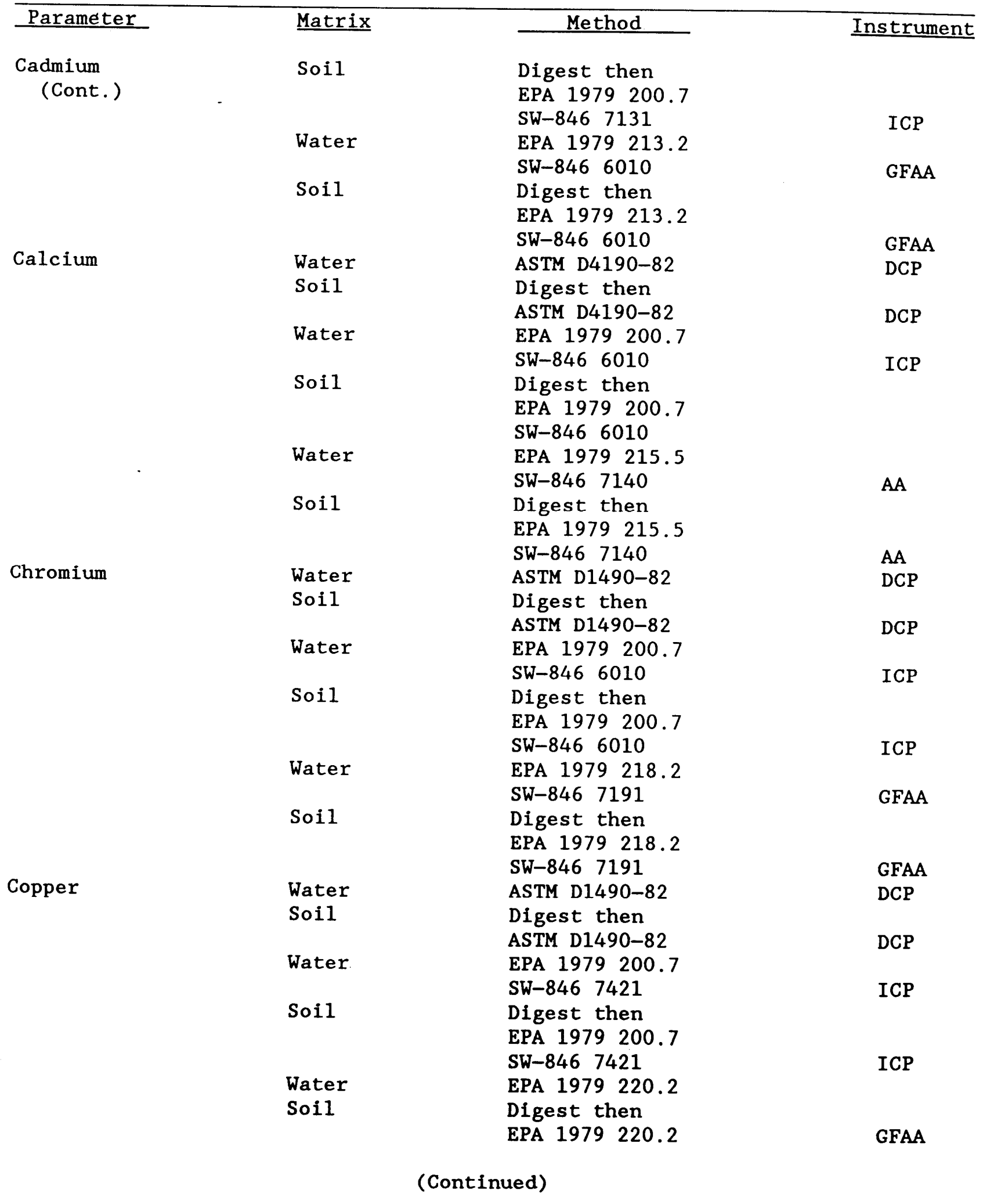


Table 3 (Continued)

\begin{tabular}{|c|c|c|c|}
\hline Parameter & Matrix & Method & Instrument \\
\hline \multirow[t]{16}{*}{ Iron } & $\begin{array}{l}\text { Water } \\
\text { Soil }\end{array}$ & $\begin{array}{l}\text { ASTM D1490-82 } \\
\text { Digest then }\end{array}$ & DCP \\
\hline & & ASTM D1490-82 & DCP \\
\hline & Water & EPA 1979200.7 & \\
\hline & & SW-846 6010 & ICP \\
\hline & Soil & Soil Digest & \\
\hline & & $\begin{array}{l}\text { EPA } 1979200.7 \\
\text { SW-846 } 6010\end{array}$ & ICP \\
\hline & Water & EPA 1979236.1 & 101 \\
\hline & & SW-846 7380 & AA \\
\hline & Soil & Digest then & \\
\hline & & EPA 1979236.1 & \\
\hline & & SW-846 7380 & AA \\
\hline & Water & EPA 1979236.2 & \\
\hline & & SW-846 7381 & GFAA \\
\hline & Soil & Digest then & \\
\hline & & EPA 1979236.2 & \\
\hline & & SW-846 7381 & GFAA \\
\hline \multirow[t]{12}{*}{ Lead } & Water & ASTM D1490-82 & DCP \\
\hline & Soil & Digest then & \\
\hline & & ASTM D1490-82 & DCP \\
\hline & Water & EPA 1979200.7 & \\
\hline & & SW-846 6010 & ICP \\
\hline & Soil & Digest then & \\
\hline & & EPA 1979200.7 & \\
\hline & & SW-846 6010 & ICP \\
\hline & Water & EPA 1979245.1 & \\
\hline & Soit & SW-846 6010 & GFAA \\
\hline & 5011 & Digest then & \\
\hline & & SW-846 6010 & GFAA \\
\hline \multirow[t]{14}{*}{ Manganese } & Water & ASTM D1490-82 & DCP \\
\hline & Soil & Digest then & \\
\hline & & ASTM D1490-82 & DCP \\
\hline & Water & EPA 1979200.7 & \\
\hline & & SW-846 6010 & ICP \\
\hline & Soil & Digest then & \\
\hline & & $\begin{array}{l}\text { EPA } 1979200.7 \\
\text { SW-846 } 6010\end{array}$ & ICP \\
\hline & Water & EPA 1979243.2 & \\
\hline & & SW-846 7460 & AA \\
\hline & Soil & Digest then & \\
\hline & & $\begin{array}{l}\text { EPA } 1979243.2 \\
\text { SW-846 } 7460\end{array}$ & AA \\
\hline & Water & EPA 1979243.1 & GFAA \\
\hline & Soil & Digest then & \\
\hline & & EPA 1979243.1 & GFAA \\
\hline
\end{tabular}


Table 3 (Continued)

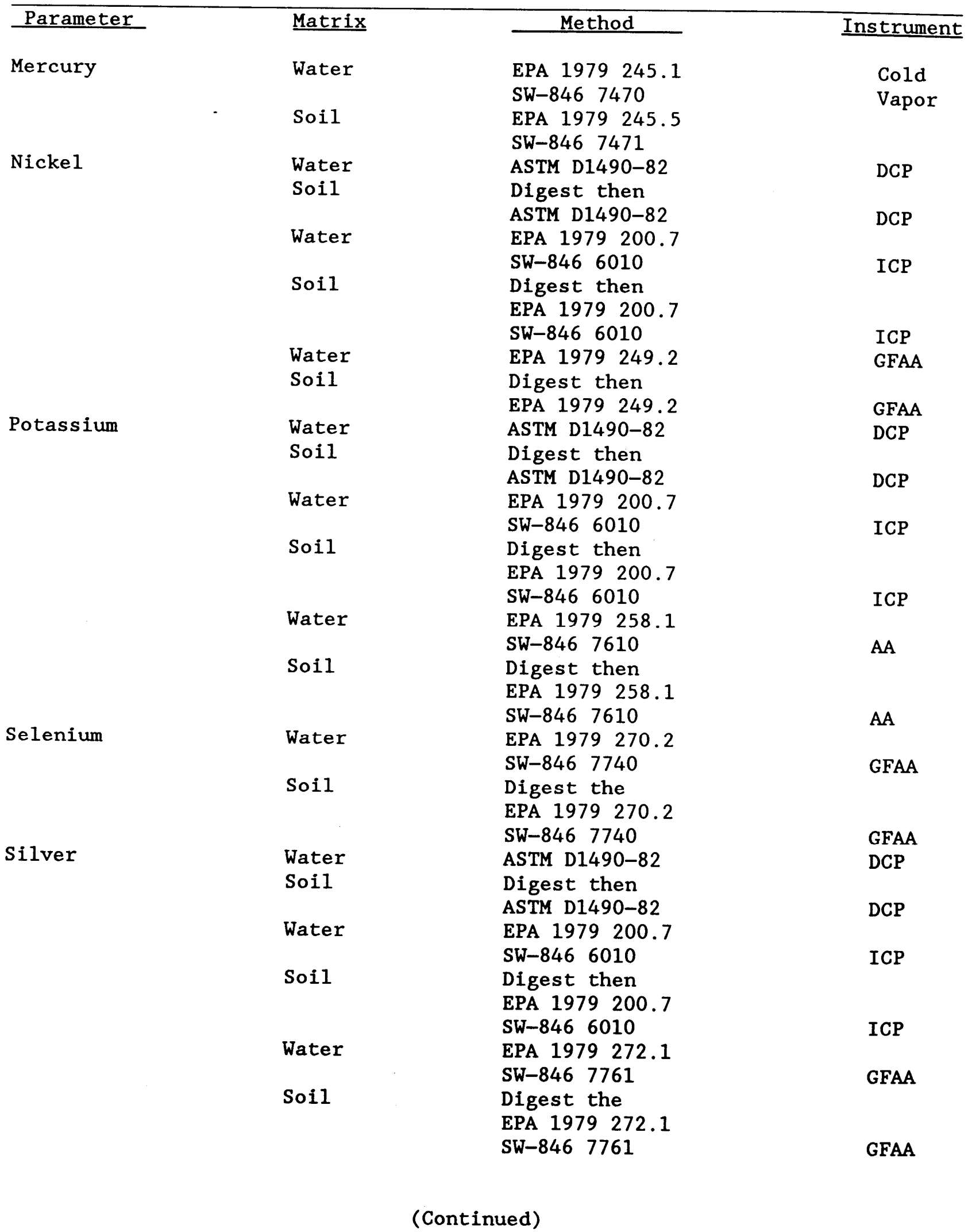


Table 3 (Concluded)

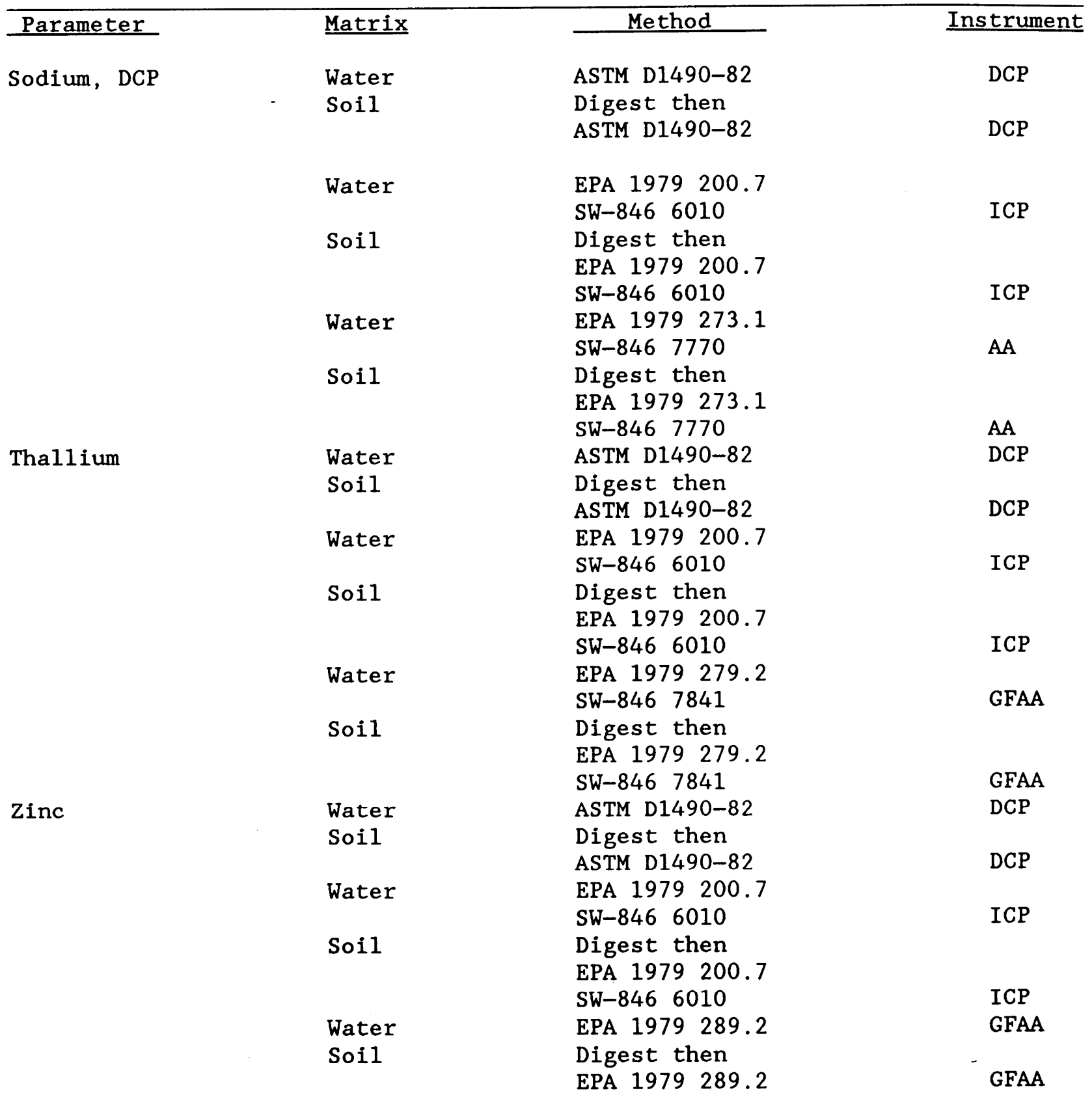


Table 4

Analytical Methods

Miscellaneous

\begin{tabular}{|c|c|c|c|}
\hline Parameter & Matrix & Methods* & Instrument \\
\hline Ammonia-N & Water & EPA 1979350.1 & $\begin{array}{l}\text { Technicon } \\
\text { Autoanalyzer }\end{array}$ \\
\hline Kjeldah1-N & Water & EPA 1979351 & $\begin{array}{l}\text { Technicon } \\
\text { Autoanalyzer }\end{array}$ \\
\hline $\begin{array}{l}\text { Nitrate- } \\
\quad \text { Nitrite-N }\end{array}$ & Water & EPA 1979353.2 & $\begin{array}{l}\text { Technicon } \\
\text { Autoanalyzer }\end{array}$ \\
\hline Total Phosphate & Water & EPA 1979365.4 & $\begin{array}{l}\text { Technicon } \\
\text { Autoanalyzer }\end{array}$ \\
\hline Orthophosphate & Water & EPA 1979365.1 & $\begin{array}{l}\text { Technicon } \\
\text { Autoanalyzer }\end{array}$ \\
\hline $\begin{array}{l}\text { Dissolved Oxygen } \\
\text { pH Value }\end{array}$ & Water & $\begin{array}{l}\text { Std Methods } 421 F \\
\text { Std Methods } 423\end{array}$ & $\begin{array}{l}\text { YSI Model } 57 \\
\text { Fisher Scientific } \\
\text { pH Meter }\end{array}$ \\
\hline Suspended Solids & Water & Std Methods 209C & $\begin{array}{l}\text { VWR Scientific } \\
\text { Oven }\end{array}$ \\
\hline $\begin{array}{c}\text { Volatile Suspended } \\
\text { Solids (VSS) }\end{array}$ & Water & Std Methods 209D & $\begin{array}{l}\text { Thermolyne } \\
\text { Furnatrol } \\
\text { Furnace }\end{array}$ \\
\hline $\begin{array}{l}\text { Cation Exchange } \\
\text { Capacity (CEC) }\end{array}$ & Soils & EPA SW-846-9080 & $\begin{array}{l}\text { Technicon } \\
\text { Autoanalyzer }\end{array}$ \\
\hline
\end{tabular}

* Std Methods - American Public Health Association, Standard Methods for Examination of Water and Wastewater 16th Edition, 1985. 1015 Fifteenth Street NW, Washington, DC 20005. EPA-SW 846. Third Edition, November 1986 with December 1988 revisions Office of Solid Waste and Emergency Response. Washington, DC 20460. EPA 600-4-79-020 - EPA Methods for Water and Waste. EPA 1979 - USEPA, Methods of Chemical Analysis of Water and Wastes EPA 600/4-79-020, March 1979 and EPA 600/4-82-055, Dec. 1982. Environmental Monitoring and Support Laboratory, Cincinnati, OH 45268. 
APPENDIX A:

IN SITU IMPLEMENTATION CASE STUDIES 


\title{
SUMMARY OF IN SITU BIOREMEDIATION CASE STUDIES
}

by

Robert D. Sproull

Department of Chemical Engineering

Oregon State University

Corvallis, OR 97331-2702

for

\author{
Environmental Laboratory \\ Waterways Experiment Station \\ Corps of Engineers \\ Department of the Army \\ Vicksburg, MS 39180-0631
}

November 30,1990

\author{
Contract No. DAA, 03-86-D-0001 \\ Delivery Order 2125 \\ Scientific Services Program
}

The views, opinions, and/or findings contained in this report are those of the author and should not be construed as an official Department of the Army position, policy, or decision, unless so designated by other documentation. 


\section{NOMENCLATURE AND ACRONYMS}

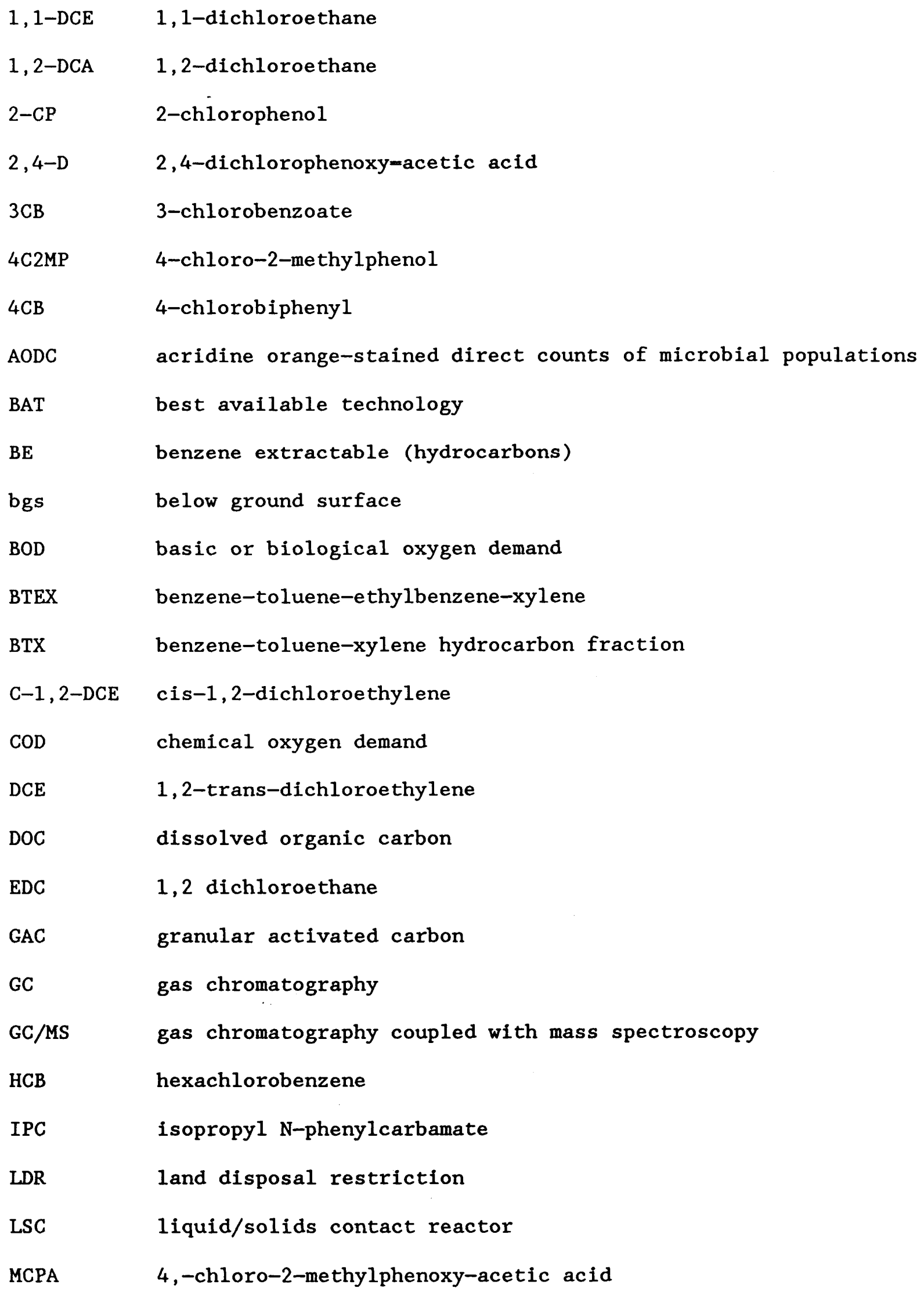




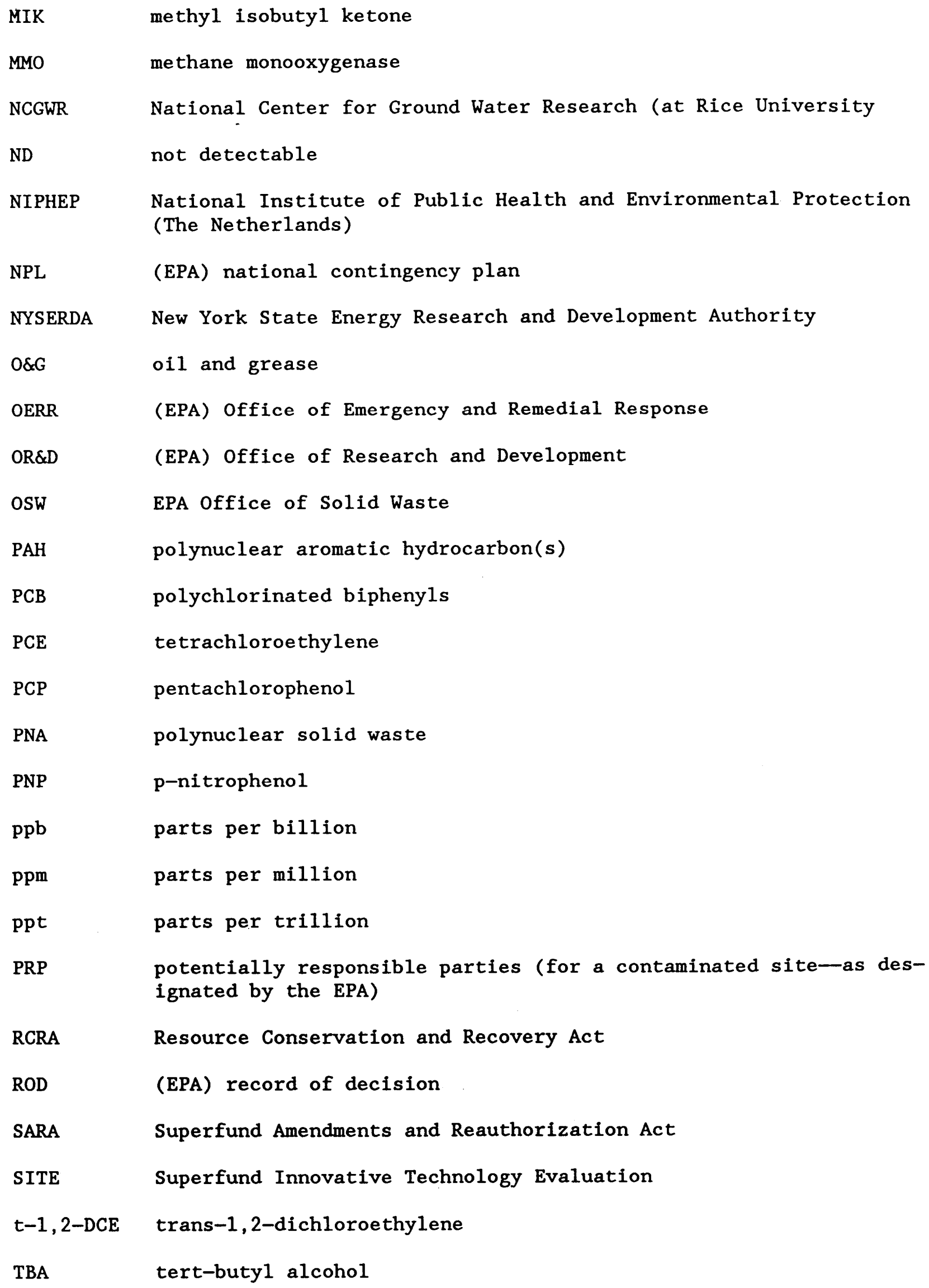




$\begin{array}{ll}\text { TCA } & 1,1,1-\text { trichloroethane } \\ \text { TCE } & 1,1,2 \text {-trichloroethylene } \\ \text { TCE } & \text { trichloroethylene } \\ \text { TDS } & \text { total dissolved solids } \\ \text { TLO } & \text { trace level organic contaminant } \\ \text { TPH } & \text { total petroleum hydrocarbons } \\ \text { TTO } & \text { total toxic organics } \\ \text { UHWS } & \text { uncontrolled hazardous waste sites } \\ \text { UOD } & \text { ultimate oxygen demand } \\ \text { VC } & \text { vinyl chloride }\end{array}$




\section{Inger Oil Refinery}

Location: The East bank of the Mississippi River near Darrow, LA.

Description of Site: An oil reclamation facility where waste oils brought to the site by barge and truck were processed in cracking towers with the final products transported from the facility by truck. As part of the plant operations between 1967 and 1978, sludges were stored in large, open lagoons and/or buried in shallow pits.

Date and Cause of Contamination: In March of 1978, when unloading a barge of used oil, a shut-off valve failure or human error led to overtopping a tank and its containment area. As a result, 16 acres of the surrounding area, including a 7.5-acre swamp, were contaminated with a mixture of refinery, motor, and lubricating oils.

Extent of the Contamination: As is typical of waste oils, pollutants such as benzene, toluene, and PAHs were present. However, no PCBs were found; and only low levels of chlorinated hydrocarbons and heavy metals were detected. Trace amounts of contaminants had migrated through the site soil to a depth of $20 \mathrm{ft}$ or more, and were also detected in the groundwater to a depth of $75 \mathrm{ft}$. Continued vertical and horizontal migration of contaminants is anticipated. GC and GC/MS were used to detect and quantify 24 different PNAs and 22 aliphatic hydrocarbons.

Hydrogeology of the Site: The soil at the site predominantly consists of silty and sandy clays, silts, and fine sands to a depth of about $120 \mathrm{ft}$. It has a permeability of about $10^{-5} \mathrm{~cm} / \mathrm{sec}$.

Government Action Taken: Failure by the owner of the refinery to clean up the site resulted in a June 1981 formal declaration by the Louisiana Environmental Control Commission that the site was abandoned. The state and regional environmental agencies designated the facility a CERCLA/SARA site. Then in April of 1982 , the site was targeted as a priority site for Superfund assistance, scoring the highest of five qualifying sites in Louisiana with a 48.98 on the EPA Superfund list.

Cleanup Treatment Methods Employed: Field studies suggested the use of a modified biotreatment approach, which was approved by state and federal environmental agencies.

Microorganisms Used for Bioremediation: A commercial inoculum obtained from Microbe Masters, Inc., in Baton Rouge, LA, with the ability to biodegrade PNAs was compared with the indigenous microflora in field studies. The commercial inoculum used in this study is technically viable in achieving acceptable rates of toxicant biotransformation and had a significant kinetic advantage in degrading toxicants over the adapted indigenous microflora. The indigenous populations were effective in toxicant biotransformation but required a significant acclimation period - toxicant half-lives were about double for the indigenous populations as compared with the commercial bacterial blend.

Results of Field Study: Based on the successful field trials, a decision was made to continue with site remediation using a modified biotreatment approach per approval of state and federal environmental agencies. In situ bioremediation is to be used in conjunction with pump-and-treat. 
Future Outlook: No estimate on how long it will take to clean up the site is available. However, postclosure monitoring of soils and leachate collected from the site was recommended for a period of 30 years following the completion of soil bioremediation.

\section{Reference:}

Portier, R. J. 1989. "Bioremediation Using Adapted Bacterial CulturesTopic 1: Examination of Site Data and Discussion of Microbial Physiology with Regard to Site Remediation," Superfund '89. Proceeds of the 10th National Conference. 
2. Gasoline Spill in Northern Michigan

(Groundwater Technology, Inc., 1985)

Location: Northern Michigan.

Description of Site: Contaminated aquifer.

Date and Cause of Contamination: In 1984, a dissolved hydrocarbon plume was discovered.

Extent of Contamination: One and a half acres were contaminated by the loss of approximately $500 \mathrm{gal}$ of gasoline.

Hydrogeology: Sorted sands to silty sands constitute most of the contaminated area. An aquitard is located $20 \mathrm{ft}$ below the aquifer surface. The groundwater flows at a rate of 2 to $4 \mathrm{ft}$ per day.

Action Taken: A 3-month pilot study was undertaken to examine the possibility of using bioremediation to clean up the site. Twelve monitoring wells, a recovery well, and an infiltration gallery were installed. Groundwater was tested on a weekly basis. Samples were measured for inorganic chemical nutrients, microbiological changes, and dissolved hydrocarbon concentrations.

Microorganisms Used: Indigenous only.

Nutrients Added: Between the pumping well and infiltration gallery, a nutrient mix tank was set up.

Oxygen Source: Because of a low background oxygen concentration ( $3.5 \mathrm{ppm}$ ), air sparging was initiated. After 4 weeks of sparging, a 100-percent increase in oxygen level was observed. Mixing of the nutrients also aided in increasing the dissolved oxygen level.

Other Treatment Methods: None.

Results: After the first month of the pilot study, aerobic bacteria had increased two orders in magnitude. By the end of the second month, the hydrocarbon plume had reduced from 65,000 to $25,000 \mathrm{ft}^{2}$. By the end of the pilot study, the hydrocarbon plume was reduced to half an acre, and the microbial count had multiplied 100 fold. The pilot study was deemed successful, approximately $100 \mathrm{gal}$ of contaminants were reduced. The biodegradation process is still continuing, and if the groundwater discharged application is approved, it is hoped the bioremediation process will fully clear up the site.

Reference: Rodecki, Edward A., Matson, Charlton, and Brenoel, Michael R., Enhanced Natural Degradation of a Shallow Hydrocarbon Contaminated Aquifer, Groundwater Technology, Inc., Michigan. 
Location: Unknown.

Description of the Site: A local municipal pump station in a rural area.

Date and Cause of the Contamination: Date unknown. The cause of the contamination was a ruptured pipeline carrying high octane gasoline.

Extent of the contamination: Approximately 134,000 gal of high octane gasoline were lost through the ruptured pipeline. The impacted area covered $270,000 \mathrm{ft}^{2}$, including a municipal water wel1. An estimated 46,000 gal of the gasoline were absorbed by the soil, and $\approx 10$ to 20 gal were dissolved in the groundwater; this resulted in a groundwater gasoline concentration of 5 to 8 ppm.

Hydrogeology: The site consisted of fractured dolomite with the groundwater at a depth of about $300 \mathrm{ft}$.

Action Taken: Approximately 88,000 gal of free gasoline were recovered over a 11-month period through 46 wells drilled at the site. In situ bioreclamation was selected as the method of remediation for the remaining 46,000 gal of gasoline trapped in the fractured dolomite at the site. Water was circulated through the site at an average rate of 70 to $100 \mathrm{gpm}$.

Microorganisms Used: Indigenous only.

Nutrients Added: Eighty-seven tons of unspecified inorganic nutrients were batch fed to the aquifer over a 12-month period.

Oxygen Source: Ten 2.5-CFM air sparger wells provided continuous aeration of the site during the addition of nutrients.

Other Treatment Methods: Apparently the circulated groundwater was treated above ground before reinjection.

Results: The production wells groundwater contamination only fell to $\approx 2.4 \mathrm{ppm}$ of gasoline during the nutrient addition period. However, biological activity continued after nutrient addition stopped. Six months later the hydrocarbon level dropped to nondetectable levels ( $<500 \mathrm{ppb}$, using $\mathrm{UV}$ analysis). Overall, free product recovery for 11 months, followed by 18 months of bioreclamation resulted in a successful cleanup of the site.

Reference: Brown, R. A., Norris, R. D., and Brubaker, G. R., Pollution Engineering, 25 (Nov. 1985). 
4. Gasoline Leakage from an Underground Storage Tank

(FMC, date unknown)

Location: Unknown.

Description of the Site: Residential area. Petroleun fumes were detected in four private residences.

Date and Cause of the contamination: Date unknown. The cause of the contamination was a leaking underground gasoline storage tank at a neighborhood service station.

Extent of the Contamination: Approximately 25,000 to 30,000 gal of gasoline were lost through the leaking underground storage tank. The impacted area covered 75,000 $\mathrm{ft}^{2}$ and included four homes, which had to be purchased by the company responsible for the contamination. An estimated 10,000 gal of gasoline were absorbed by the soil in the area around the service station, and $\approx 333$ gal were dissolved in the groundwater; this resulted in a groundwater gasoline concentration of 30 to $40 \mathrm{ppm}$. Soil analysis at the site indicated contaminant levels of 2,000 to $3,000 \mathrm{ppm}\left(0.04\right.$ to $\left.0.06 \mathrm{gal} / \mathrm{ft}^{3}\right)$ at 3 to $8 \mathrm{ft}$ above the water table.

Hydrogeology: The site consisted of $20 \mathrm{ft}$ of course grain sand/fine gravel underlaid with clay. The water table ranged from 8 to $15 \mathrm{ft}$ below the surface.

Action Taken: Over a 2-year period 18,500 gal of free gasoline were recovered through five pumping wells drilled at the site. The average pumping rates of the water were 18 to $40 \mathrm{gpm}$.

Microorganisms Used: Indigenous only.

Nutrients Added: Nutrients were periodically injected over a 10-month period through 14 wel1s.

Oxygen Source: During the 10 months of periodic nutrient injection, air was continuously sparged through the 14 injection wells.

Other Treatment Methods: Apparently the circulated groundwater was treated above ground before reinjection.

Results: During the 10-month period of nutrient and oxygen injection, the gasoline level was reduced to less than $50 \mathrm{ppm}$ in the soil and to less than 1 $\mathrm{ppm}$ in the groundwater. As a result of the successful in situ bioremediation program, the four residences, which had to be purchased because of the fume problem, were resold.

Air sparging was only able to maintain dissolved oxygen levels of 1 to 2 ppm in the area of the spill. However, during the course of the bioremediation program, a test was conducted to compare the efficiency of hydrogen peroxide injection with air sparging. During this test peroxide was dissolved and injected with the nutrients. The subsequent rise in dissolved oxygen levels to over $15 \mathrm{ppm}$ helps to establish the process of using peroxide to increase D.O. levels, and thereby enhance bioremediation. 
Reference: Brown, R. A., Norris, R. D., and Brubaker, G. R., Pollution Engineering, 25 (Nov. 1985). 
5. Oil Spill in the Upper Rhine Valley

Location: Upper Rhine Valley.

Description of Site: Industrial area of a city.

Date and Cause of Contamination: Date unknown. Aromatic hydrocarbons were detected at an oil spill site. These hydrocarbons had been used in the area 5 years prior to the spill.

Extent of Contamination: The hydrocarbons in the soil amounted to about 30 tons. They were detected up to $300 \mathrm{~m}$ from the sight, and also in a shallow aquifer $5 \mathrm{~m}$ below the surface.

Hydrology: The shallow aquifer, $5 \mathrm{~m}$ below the surface, contained silt and clay up to $10 \mathrm{~m}$ thick. Below the aquifer, a layer of clay around $20 \mathrm{~m}$ thick exists. The permeability of the aquifer is about $5 \times 10^{-4} \mathrm{~m} / \mathrm{sec}$.

Action Taken: Restoration of the site combined a 2-year process of flushing and microbial degradation in the subsurface. Twenty-one wells were used: twelve injection wells and nine intake wells. Four of the injection wells were used as protection wells. Uncontaminated water was injected through the protection wells and into the groundwater, thus preventing contaminated water from spreading. The other 12 injection wells were used as flush wells.

Water, withdrawn from the intake wells, was filtered, heated to $20^{\circ}$, enriched with nutrients and nitrate, and recirculated back into the subsurface through the flush wells. The infiltration rate was around $10 \mathrm{l} / \mathrm{sec}$ for the protection wells and $5 \mathrm{l} / \mathrm{sec}$ for the flush wells.

Microorganisms Used: Indigenous only.

Nutrients Added: Nutrients were added to the flush water. About $1 \mathrm{mg} / \ell$ of ammonia and $0.3 \mathrm{mg} / \ell$ of orthophosphate were added.

Oxygen Source: Aeration was used to saturate the flushwater with oxygen. Nitrate, an oxygen donor, was added to the flushwater at a maximum rate of $500 \mathrm{mg} / \mathrm{l}$.

Other Treatment Methods: The groundwater was treated above ground. The flushwater was filtered to remove iron manganese and dissolved hydrocarbons.

Results: Soil samples, analyzed every 4 months, indicated a reduction of hydrocarbon content in the soil. Heating the flushwater to $20^{\circ}$ doubled the nitrate degradation rate. After 10 months, the concentration of the aliphatic hydrocarbons was reduced to one-third its original value. The aromatic hydrocarbons, benzene and toluene, were completely removed within 8 months. After successfully completing the entire restoration project, 100 tons of nitrate had been used for the biodegradation of the 30 tons of hydrocarbons.

Reference: Batterman, G. 1986. "Decomposition of Polluted Aquifers by Biodegradation," Contaminated Soll, Martinus Nyhoff Pub., Boston, MA. 
6. Pipeline Leak in Amber, PA.

Location: Amber, PA.

Site Description: Unknown.

Date and Cause of Contamination: A pipeline leaked gasoline.

Extent of Contamination: Leakage of the pipeline resulted in over 100,000 gal of a high octane gasoline being spilled. A pump-and-treat method removed approximately 63,000 gal, and the remaining residual gasoline was degraded using a biostimulated process.

Hydrogeology: The contaminated site consisted of a highly fractured dolomite outcrop with quartzite lying below. The water table was around 9.2 to $30.5 \mathrm{~m}$ below the surface.

Action Taken: Forty-six monitoring wells were installed. A pump-and-treat method was undertaken to remove the bulk of the gasoline from the groundwater. Once the dissolved gasoline concentration in the groundwater was less than $5 \mathrm{ppm}$, a biostimulation process was initiated.

Biodegradation of the hydrocarbons was stimulated by adding nutrients on a batch basis to the aquifer. Oxygen was supplied to the groundwater by sparging air through the monitoring wells.

Microorganisms Used: Indigenous bacteria were used. The bacteria that were identified at the site include species of the genera: Nocardia, Microccus, Acinetobactus, Florobacterium, and Pseudomonas. It was believed the Nocardin were responsible for degradation of the aliphatic hydrocarbons, and Pseudomonas degraded the aromatic.

Nutrients Added: A 30-percent concentration of nutrients were batch fed into the aquifer. The nutrients included ammonium sulfate, disodium phosphate, and monosodium phosphate.

Oxygen Addition: Oxygen was sparged into the wells at a rate of $0.06 \mathrm{~m}^{3} / \mathrm{min}$.

Other Treatment Methods: A pump-and-treat method was first used to remove 63,000 gal of gasoline.

Results: The pump-and-treat method reduced the hydrocarbon levels in the groundwater to less than $5 \mathrm{ppm}$. Approximately 31,500 gal of gasoline remained.

For biostimulation, an estimated $44 \mathrm{~g}$ of nitrogen, $22 \mathrm{~g}$ of phosphorous, and $730 \mathrm{~g}$ of oxygen was needed to degrade $1 \ell$ of gasoline. Once nutrient addition was begun at the site, bacterial counts increased from $10^{3}$ to $10^{7} \mathrm{ce} 1 \mathrm{ls} / \mathrm{ml}$. The gasoline concentration remained the same in the groundwater during nutrient addition. However, after 10 months following the termination of the biostimulation process, no gasoline was detected in the groundwater and a 100-fold increase in bacteria was observed. An estimated 23,000 to $30,000 \mathrm{gal}$ of gasollne were degraded. 
Reference: Lee, M. D., Thomas, J. M., Borden, K. C., Bedient, P. B., Wilson, J. J., and Ward, C. H. 1988. "Biorestoration of Aquifers Contaminated with Organic Compounds," CRC Critical Reviews in Environmental Control, Vol 18, No. $1, \mathrm{p} 34$. 
7. Petrochemical Dumpsite Cleanup*

(Resource Engineering, date unknown)

Location: Near Houston, TX.

Description of Site: Residential area. A lagoon that was used as a petrochemical dumpsite.

Date and Cause of Contamination: Date unknown. The site was used as a petrochemical dump. After the contractor, French Ltd., went out of business, the site had to be cleaned up.

Extent of Contamination: The contaminated area was a 7.3-acre lagoon. Toxic petrochemical by-products and other compounds contaminated an estimated $70,000 \mathrm{cu}$ yd of soil and sand and $80,000 \mathrm{cu}$ yd of sludge. The water above the sludge layer had a hydrocarbon concentration of $200 \mathrm{ppm}$.

Action Taken: A group called the Potentially Responsible Parties (PRP) was formed to clean up the site. An initial bioremediation test was performed on one-third of an acre in the contaminated area. Six weeks after aeration of the area began, natural bacteria started growing. At this point, a sludge pump was installed to increase the rate of mixing. After 4 months of aeration, a suction dredge was also added to the site to further increase mixing.

Hydrogeology: Soil and sand.

Microorganisms Used: Indigenous only.

Nutrients Added: Liquid fertilizer was added during the initial weeks of the test.

Oxygen Source: Aeration of the water and sludge provided oxygen. Four compressors were used to pump $1,600 \mathrm{cu} \mathrm{ft} / \mathrm{min}$ of air through two separate sets of aerators.

Other Treatment Methods: Only bioremediation was used in the pilot study.

Results: During the first 4 months of aeration, the contaminated sludge had been consumed. Three months after the cutter dredge was operating, the hydrocarbon concentration in the water was $5 \mathrm{ppm}$. The test area was considered cleaned up. An estimated $31 / 2$ years of bioremediation would be needed to clear the entire contaminated lagoon.

Reference: Savage, Peter. 1987 (Nov). "Bacteria Pass a Houston Cleanup Test," Chemical Week, pp 55-56.

* Also summarized in The Netherlands NIPHEP Report No. 738708006, Case No. U8. 


\section{An Aquifer Contaminated With Hydrocarbons}

Location: Millville, NJ .

Hydrology: Highly permeable sand characterized the contaminated aquifer.

Action Taken: A biostimulation process was used to clean up the contaminated aquifer. Nutrients and aerated water were initially injected into the aquifer; however, the oxygen content was too low to meet microbial demand. Additional aeration was implemented by air sparging, and eventually the cleanup met state requirements.

Microorganisms Used: Indigenous.

Nutrients Added: Nutrients were added at rates of 2.4 to $4.2 \mathrm{ft} /$ day.

Oxygen Added: Aeration of the system was accomplished first by adding aerated water, then sparging with oxygen.

Other Treatment Methods: None specified.

Results: Initially the dissolved oxygen was consumed; however, analysis of materials from wells in the contaminated site indicated the gasoline concentration was relatively unchanged. The low oxygen levels resulted in microbial formation of phenols, which upon additional aeration, decreased. With increased aeration, the site eventually met cleanup requirements, and an estimated 10 to 1,000-fold increase in bacteria was observed.

Reference: Lee, M. D., Thomas, J. M., Borden, R. C., Bedient, P. B., Wilson, J. T., and Ward, C. H. 1988. "Biorestoration of Aquifers Contaminated with Organic Compounds," CRC Critical Reviews in Environmental Control, Vol 18, No. $1, \mathrm{p} 38$. 
9. Gasoline Spill in La Grange, OR

Location: La Grange, Oregon.

Description of Site: Vapors resulting from a gasoline spill were discovered in two restaurants.

Action Taken: In situ bioremediation and a vapor cleanup process were used to decontaminate the site. The biodegradation process involved recirculation of groundwater which was aerated and enriched in nutrients.

Results: Concentrations of gasoline after 7 months of enhanced bioremediation were around 100 to $500 \mathrm{ppm}$. The dissolved organic carbon was at $20 \mathrm{ppm}$ in the groundwater. The level of dissolved organic carbon was less than $5 \mathrm{ppm}$ after an additional 3 months.

Reference: Lee, M. D., Thomas, J. M., Borden, R. C., Bedient, P. B., Wilson, J. T., and Ward, C. H. 1988. "Biorestoration of Aquifers Contaminated with Organic Compounds," CRC Critical Reviews in Environment Control, Vol 18, No. 1, p 36. 
10. Service Station Spill Above and Below Ground (Groundwater Technology, Inc.)

Location: Suburb of Montreal, Canada.

Description of Site:- Suburban area. Petroleum vapors were discovered in a warehouse located across the street from a service station.

Date and Cause of Contamination: Date unknown. Over a long period of time, petroleum leaked from service pumps and underground storage tanks at a service station.

Extent of Contamination: The spill site was divided into four different areas labeled A through D. Site A, the southern most pump island of the station, had a volatile organic chemicals (VOC) concentration of $15,000 \mathrm{ppb}$. The initial concentration level of VOC around the tank pit, site B, was $130 \mathrm{ppb}$. Area $C$, the land between the station and warehouse, had a VOC concentration around 30 to $35 \mathrm{ppb}$. The basement of the warehouse, where the vapors were discovered, area D, had a VOC level of 15,000 ppb.

Hydrogeology: Information not given.

Action Taken: Two separate bioremediation processes were set up: one at the service station, and the other at the warehouse across the street. The cleanup of the service station involved using the recharge galleries and an inceptor trench. One of the recharge galleries was located by the pump island, and the other by the ask pit. Groundwater was extracted from the trench, which swept across the front of the station, and treated above ground. The water, prior to reinjection back into the subsurface, was enriched with a 10-percent peroxide solution and nutrients. The nutrients were added on a batch basis. Groundwater was redirected into a 300-gal tank, nutrients added, then pumped back to the galleries. The amended water entered the subsurface around 3 to $5 \mathrm{gpm}$. The total oxygen delivered to the contaminated soil was approximately $22 \mathrm{~kg}$ per day.

The warehouse cleanup involved six injection wells, a recovery well, and a French drain sump. Hydrogen peroxide was added to the groundwater at a concentration of $2,000 \mathrm{ppm}$. The groundwater and nutrients were circulated at approximately $4 \mathrm{gpm}$.

Microorganisms Used: Indigenous only.

Nutrients Added: Ammonia and phosphate salts were added to the groundwater on a batch basis. A 300-gal tank was used to dilute the nutrients. The nutrient-enriched water was then metered into the recharge galleries.

Oxygen Source: A dilute 10-percent peroxide solution was used. Approximately $22 \mathrm{~kg}$ of oxygen a day was added to the soil at the station. An oxygen concentration of $2,000 \mathrm{ppm}$ was added to the soil under the warehouse.

Other Treatment Methods: Groundwater was treated above.

Results: At site A, treatment of the groundwater resulted in a reduced VOC leve1 of $1,300 \mathrm{ppb}$. Injection of oxygen and nutrients further reduced the VOC level, after 6 months, to less than $100 \mathrm{ppb}$. After this period, the 
bioremediation process was terminated. Post sampling exhibited a VOC level ranging between 100 and $250 \mathrm{ppb}$.

The pump-and-treat method at site B reduced VOC levels to less than $10 \mathrm{ppm}$. Bioremediation helped to maintain the low VOC level; however, there were two sharp spikes in the concentration levels. One occurred at 16 to 18 months, and the other at 28 to 30 months. These dates correspond to low-water tables which resulted in exposed contaminants that had been imported in bedrock. Eventually groundwater levels stabilized, and the process was terminated.

Area $C$ had results comparable to those at area B. Area $C$ had spikes of high contamination, which again corresponded to low tables. The results of both areas $B$ and $C$ indicate that bioremediation effectively removes trapped contamination from bedrock.

For area D, initial vapor extraction resulted in a reduced voC level to $3,700 \mathrm{ppb}$. Upon implementation of a bioremediation process, VOC levels, after 10 months, dropped to $200 \mathrm{ppb}$. After termination of the process, the exposed VOC level was under $500 \mathrm{ppb}$, and the dissolved VOC level was less than $200 \mathrm{ppb}$.

Reference: Brown, Richard, and Tube, Richard. 1990 (May). "In Situ Physical and Biological Treatment of Volatile Organic Contamination: A Case Study Through Closure," Second Form in Innovate Hazardous Waste Treatment Technologies: Domestic and International. 
11. Leaking Tanks of Waste Fuels and Solvents (FMC)

Location: Unknown.

Description of the Site: A tank vault adjacent to an industrial facility.

Date and Cause of Contamination: Date unknown. The cause of the contamination was two leaking waste fuels/solvents storage tanks. The impacted area was confined to a $25-$ by $70-$ by $12-\mathrm{ft}$ tank vault. The leakage of an estimated 700 to $1,300 \mathrm{gal}$ of a mixture of fuels and solvents produced fumes that were detected in a nearby laboratory building. The leaked mixture consisted of approximately 45 percent aromatics and 55 percent alkanes. About half of the leaked waste was absorbed by the soil. But only a gallon or two entered the groundwater, giving it a contaminant concentration of $\approx 22$ to $45 \mathrm{ppm}$.

Hydrogeology: The tank vault was backfilled with medium pea gravel cut into clay strata (glacial till), fractured shale, and silt stone. The water table was only $4 \mathrm{ft}$ below ground level.

Action Taken: After recovery of approximately 700 gal of free product, in situ bioremediation was used to reduce soil and groundwater contamination. Water was circulated through the site at an average rate of $25 \mathrm{gpm}$.

Microorganisms Used: Indigenous only.

Nutrients Added: Sufficient nutrients were added to degrade $\approx 150$ to 400 gal of hydrocarbons in the soil.

Oxygen Source: Apparently the enhanced microbial nutrient formulation contained hydrogen peroxide.

Other Treatment Methods: No information was given about the aboveground treatment of circulated water-apparently, it was not treated before nutrient addition, and reinjection activated carbon was used in the final stage of the treatment program to remove residual hydrocarbons in the groundwater.

Results: The groundwater levels of contaminants fell from 22 to $45 \mathrm{ppm}$ to $<550 \mathrm{ppb}$ during the $21 / 2$ months of in situ bioremediation. Then, after $31 / 2$ months of treatment with activated carbon, the groundwater contaminant level fell to less than $10 \mathrm{ppb}$, and the project was terminated.

Activated carbon was used as the final stage of the remediation process for two reasons. First, the water injection rate into the vault decreased significantly because of an improper screen size and the migration of fines and silicates. This had a detrimental effect on the transport of nutrients. Second, the successful degradation of hydrocarbons absorbed to the soil left only a very small amount of residual contaminant to be treated.

Reference: Brown, R. A., Morris, R. D., and Brubaker, G. R. 1985 (Nov). Pollution Engineering, p 25. 
12. Gasoline Leakage from Aboveground Storage Tanks in Granger, Indiana (Rice University)

Location: Granger, IN.

Description of Site: Storage facility.

Date and Cause of Contamination: The leak occurred in 1977. Gasoline leaked from an aboveground service station into a shallow aquifer.

Extent of Contamination: Initial contamination levels are unknown.

Action Taken: A study was performed on a site used previously for biodegradation of contaminating hydrocarbons. The study, performed 2 years after the biostimulation process was terminated, involved analysis of three different core samples. One sample was from a contaminated area, another from an uncontaminated area, and the last from the biostimulated zone. The microbial numbers, the heterotrophic potential, and the biodegradation potential of benzene toluene, ethylbenzene, o-xylene, and m-xylene (BTEX) were determined from the core samples.

Hydrology: The contaminated aquifer was $25 \mathrm{ft}$ below the surface. The surface above the aquifer consisted of fine-grained, silty sand. The velocity of the groundwater was $0.0068 \mathrm{ft} /$ day with a hydraulic gradient of $0.002 \mathrm{ft} / \mathrm{ft}$.

Microorganisms Used: Indigenous only.

Nutrients Added: None during course of study.

Oxygen Source: In the original field demonstration, hydrogen peroxide was used.

Other Treatment Methods: Analysis of core samples was performed in a commercial laboratory.

Results: Analysis of core samples indicated that microbial counts were higher in the biostimulated zone than either of the other two areas. This, however, may have been caused by the addition of nutrients during the biostimulation treatment. Attempts were made to enhance mineralization of benzene and toluene by changing environmental conditions and nutrient status; however they were unsuccessful except for the addition of acetate. Acetate enhanced the mineralization of toluene in the contaminated core samples. Peroxide was also used for amendment of samples. It was found that for peroxide-amended samples in the biostimulated core, toluene mineralization was substantially greater than for unamended samples. The results of the laboratory investigation performed on the core samples indicate that microbial numbers and activity still remained enhanced for at least 2 years after the biostimulation process had been terminated.

Reference: Thomas, J. M. , Gordy, V. R., Forenza, S., and Ward, C. H. 1990. "Biodegradation of BTEX in Subsurface Materials Contaminated with Gasoline: Granger, Indiana," Wat. Sci., Vol 22, No. 6, pp 53-62. 
13. Jet Fuel Contamination at Eglin Air Force Base*

(Air Force Engineers, November 1986-1988)

Location: Eglin, Air Force Base, FL.

Date and Cause of Contamination: In 1984, damage to vegetation near the Eglin Air Force Base was discovered. Investigation of the problem revealed a jet fuel pipeline leak. The underground pipeline was leaking JP-4 jet fuel at a rate of about $0.3 \mathrm{gal} / \mathrm{min}$.

Extent of Contamination: An estimated 20,000 gallons of jet fuel leaked. The contamination zone encompassed about 4,000 $\mathrm{cu}$ yd of soil and a shallow aquifer. Total hydrocarbon levels in the saturated zone were around $200 \mathrm{mg} / \ell$. The jet fuel contamination consisted of aromatic hydrocarbons.

Hydrogeology: The contaminated aquifer was located just below the surface. Sand, clay, and limestone characterized the site. The aquifer was unconfined on top, yet an impermeable clay preventing spreading of the contaminants below the aquifer. The aquifer was around $40 \mathrm{ft}$ deep and had a permeability of $2.3 \times 10^{-8} \mathrm{~cm} / \mathrm{sec}$.

Initial Treatment: Both a passive skimmer recovery method and hand bailing were undertaken to remove free product contaminants from the site. Approximately 7,400 gal of hydrocarbons were removed initially.

Preliminary Laboratory Studies:

Action Taken: Initially, soil and groundwater samples were collected and tested for microbial population, contaminants, and nutrient availability. Chemical analysis, field analysis, and microbial analysis were performed to obtain the required pretesting information.

Results: Hydrogen peroxide stability was checked. First, $290 \mathrm{mg} / \ell$ of peroxide was added to a slurry containing nutrients and groundwater. Approximately 50 percent of the peroxide decomposed within $6 \mathrm{hr}$ of initial exposure. A second addition of peroxide to the sample resulted in only a 5-percent reduction. Results indicated the most efficient means of adding peroxide in the field would be a low initial start-up concentration of peroxide, then gradually building the concentration level to its maximum.

Restore 375 nutrient was used in both the laboratory studies and field demonstration. During the laboratory study, the samples receiving the nutrients displayed evidence of biodegrading capabilities on extracted fractions of the jet fuel. However, there was no discernible difference in the extent or rate of biodegradation as a result of nutrient addition. Thus, the background nutrient levels were sufficient for biodegradation at the site. The nutrient Restore 375 was also tested in the laboratory for sorptive characteristics and permeability of surface material. The chloride in the nutrient traveled fastest, while both ammonia and phosphate were retained quite significantly in the soil. Precipitation of metals in the soil with the nutrient was also tested.

* Also summarized in The Netherlands NIPHEP Report No. 738708006, Case No. U6. 


\section{An Aquifer Contaminated With Hydrocarbons}

Location: Millville, NJ .

Hydrology: Highly permeable sand characterized the contaminated aquifer.

Action Taken: A biostimulation process was used to clean up the contaminated aquifer. Nutrients and aerated water were initially injected into the aquifer; however, the oxygen content was too low to meet microbial demand. Additional aeration was implemented by air sparging, and eventually the cleanup met state requirements.

Microorganisms Used: Indigenous.

Nutrients Added: Nutrients were added at rates of 2.4 to $4.2 \mathrm{ft} /$ day.

Oxygen Added: Aeration of the system was accomplished first by adding aerated water, then sparging with oxygen.

Other Treatment Methods: None specified.

Results: Initially the dissolved oxygen was consumed; however, analysis of materials from wells in the contaminated site indicated the gasoline concentration was relatively unchanged. The low oxygen levels resulted in microbial formation of phenols, which upon additional aeration, decreased. With increased aeration, the site eventually met cleanup requirements, and an estimated 10 to 1,000-fold increase in bacteria was observed.

Reference: Lee, M. D., Thomas, J. M., Borden, R. C., Bedient, P. B., Wilson, J. T., and Ward, C. H. 1988. "Biorestoration of Aquifers Contaminated with Organic Compounds," CRC Critical Reviews in Environmental Control, Vol 18, No. $1, \mathrm{p} 38$. 
9. Gasoline Spill in La Grange, OR

Location: La Grange, Oregon.

Description of Site: Vapors resulting from a gasoline spill were discovered in two restaurants.

Action Taken: In situ bioremediation and a vapor cleanup process were used to decontaminate the site. The biodegradation process involved recirculation of groundwater which was aerated and enriched in nutrients.

Results: Concentrations of gasoline after 7 months of enhanced bioremediation were around 100 to $500 \mathrm{ppm}$. The dissolved organic carbon was at $20 \mathrm{ppm}$ in the groundwater. The level of dissolved organic carbon was less than $5 \mathrm{ppm}$ after an additiona1 3 months.

Reference: Lee, M. D., Thomas, J. M. , Borden, R. C., Bedient, P. B., Wilson, J. T., and Ward, C. H. 1988. "Biorestoration of Aquifers Contaminated with Organic Compounds," CRC Critical Reviews in Environment Control, Vol 18, No. $1, \mathrm{p} 36$. 
10. Service Station Spill Above and Below Ground (Groundwater Technology, Inc.)

Location: Suburb of Montreal, Canada.

Description of Site:- Suburban area. Petroleum vapors were discovered in a warehouse located across the street from a service station.

Date and Cause of Contamination: Date unknown. Over a long period of time, petroleum leaked from service pumps and underground storage tanks at a service station.

Extent of Contamination: The spill site was divided into four different areas labeled A through D. Site A, the southern most pump island of the station, had a volatile organic chemicals (VOC) concentration of $15,000 \mathrm{ppb}$. The initial concentration level of VOC around the tank pit, site B, was $130 \mathrm{ppb}$. Area $C$, the land between the station and warehouse, had a VOC concentration around 30 to $35 \mathrm{ppb}$. The basement of the warehouse, where the vapors were discovered, area D, had a VOC level of 15,000 ppb.

Hydrogeology: Information not given.

Action Taken: Two separate bioremediation processes were set up: one at the service station, and the other at the warehouse across the street. The cleanup of the service station involved using the recharge galleries and an inceptor trench. One of the recharge galleries was located by the pump island, and the other by the ask pit. Groundwater was extracted from the trench, which swept across the front of the station, and treated above ground. The water, prior to reinjection back into the subsurface, was enriched with a 10-percent peroxide solution and nutrients. The nutrients were added on a batch basis. Groundwater was redirected into a 300-gal tank, nutrients added, then pumped back to the galleries. The amended water entered the subsurface around 3 to $5 \mathrm{gpm}$. The total oxygen delivered to the contaminated soil was approximately $22 \mathrm{~kg}$ per day.

The warehouse cleanup involved six injection wells, a recovery well, and a French drain sump. Hydrogen peroxide was added to the groundwater at a concentration of $2,000 \mathrm{ppm}$. The groundwater and nutrients were circulated at approximately $4 \mathrm{gpm}$.

Microorganisms Used: Indigenous only.

Nutrients Added: Ammonia and phosphate salts were added to the groundwater on a batch basis. A 300-gal tank was used to dilute the nutrients. The nutrient-enriched water was then metered into the recharge galleries.

Oxygen Source: A dilute 10-percent peroxide solution was used. Approximately $22 \mathrm{~kg}$ of oxygen a day was added to the soil at the station. An oxygen concentration of $2,000 \mathrm{ppm}$ was added to the soil under the warehouse.

Other Treatment Methods: Groundwater was treated above.

Results: At site A, treatment of the groundwater resulted in a reduced VOC leve1 of $1,300 \mathrm{ppb}$. Injection of oxygen and nutrients further reduced the VOC level, after 6 months, to less than $100 \mathrm{ppb}$. After this period, the 
bioremediation process was terminated. Post sampling exhibited a VOC level ranging between 100 and $250 \mathrm{ppb}$.

The pump-and-treat method at site $B$ reduced VOC levels to less than $10 \mathrm{ppm}$. Bioremediation helped to maintain the low VOC level; however, there were two sharp spikes in the concentration levels. One occurred at 16 to 18 months, and the other at 28 to 30 months. These dates correspond to low-water tables which resulted in exposed contaminants that had been imported in bedrock.

Eventually groundwater levels stabilized, and the process was terminated.

Area $C$ had results comparable to those at area $B$. Area $C$ had spikes of high contamination, which again corresponded to low tables. The results of both areas $B$ and $C$ indicate that bioremediation effectively removes trapped contamination from bedrock.

For area D, initial vapor extraction resulted in a reduced VoC level to $3,700 \mathrm{ppb}$. Upon implementation of a bioremediation process, VOC levels, after 10 months, dropped to $200 \mathrm{ppb}$. After termination of the process, the exposed VOC level was under $500 \mathrm{ppb}$, and the dissolved VOC level was less than $200 \mathrm{ppb}$.

Reference: Brown, Richard, and Tube, Richard. 1990 (May). "In Situ Physical and Biological Treatment of Volatile Organic Contamination: A Case Study Through Closure," Second Form in Innovate Hazardous Waste Treatment Technologies: Domestic and International. 
11. Leaking Tanks of Waste Fuels and Solvents (FMC)

Location: Unknown.

Description of the Site: A tank vault adjacent to an industrial facility.

Date and Cause of Contamination: Date unknown. The cause of the contamination was two leaking waste fuels/solvents storage tanks. The impacted area was confined to a $25-$ by $70-$ by $12-\mathrm{ft}$ tank vault. The leakage of an estimated 700 to $1,300 \mathrm{gal}$ of a mixture of fuels and solvents produced fumes that were detected in a nearby laboratory building. The leaked mixture consisted of approximately 45 percent aromatics and 55 percent alkanes. About half of the leaked waste was absorbed by the soil. But only a gallon or two entered the groundwater, giving it a contaminant concentration of $\approx 22$ to $45 \mathrm{ppm}$.

Hydrogeology: The tank vault was backfilled with medium pea gravel cut into clay strata (glacial till), fractured shale, and silt stone. The water table was only $4 \mathrm{ft}$ below ground level.

Action Taken: After recovery of approximately 700 gal of free product, in situ bioremediation was used to reduce soil and groundwater contamination. Water was circulated through the site at an average rate of $25 \mathrm{gpm}$.

Microorganisms Used: Indigenous only.

Nutrients Added: Sufficient nutrients were added to degrade $\approx 150$ to 400 gal of hydrocarbons in the soil.

Oxygen Source: Apparently the enhanced microbial nutrient formulation contained hydrogen peroxide.

Other Treatment Methods: No information was given about the aboveground treatment of circulated water-apparently, it was not treated before nutrient addition, and reinjection activated carbon was used in the final stage of the treatment program to remove residual hydrocarbons in the groundwater.

Results: The groundwater levels of contaminants fell from 22 to $45 \mathrm{ppm}$ to $<550 \mathrm{ppb}$ during the $21 / 2$ months of in situ bioremediation. Then, after $31 / 2$ months of treatment with activated carbon, the groundwater contaminant level fell to less than $10 \mathrm{ppb}$, and the project was terminated.

Activated carbon was used as the final stage of the remediation process for two reasons. First, the water injection rate into the vault decreased significantly because of an improper screen size and the migration of fines and silicates. This had a detrimental effect on the transport of nutrients. Second, the successful degradation of hydrocarbons absorbed to the soil left only a very small amount of residual contaminant to be treated.

Reference: Brown, R. A., Morris, R. D., and Brubaker, G. R. 1985 (Nov). Pollution Engineering, p 25. 
12. Gasoline Leakage from Aboveground Storage Tanks in Granger, Indiana (Rice University)

Location: Granger, IN.

Description of Site: Storage facility.

Date and Cause of Contamination: The leak occurred in 1977. Gasoline leaked from an aboveground service station into a shallow aquifer.

Extent of Contamination: Initial contamination levels are unknown.

Action Taken: A study was performed on a site used previously for biodegradation of contaminating hydrocarbons. The study, performed 2 years after the biostimulation process was terminated, involved analysis of three different core samples. One sample was from a contaminated area, another from an uncontaminated area, and the last from the biostimulated zone. The microbial numbers, the heterotrophic potential, and the biodegradation potential of benzene toluene, ethylbenzene, o-xylene, and m-xylene (BTEX) were determined from the core samples.

Hydrology: The contaminated aquifer was $25 \mathrm{ft}$ below the surface. The surface above the aquifer consisted of fine-grained, silty sand. The velocity of the groundwater was $0.0068 \mathrm{ft} /$ day with a hydraulic gradient of $0.002 \mathrm{ft} / \mathrm{ft}$.

Microorganisms Used: Indigenous only.

Nutrients Added: None during course of study.

Oxygen Source: In the original field demonstration, hydrogen peroxide was used.

Other Treatment Methods: Analysis of core samples was performed in a commercial laboratory.

Results: Analysis of core samples indicated that microbial counts were higher in the biostimulated zone than either of the other two areas. This, however, may have been caused by the addition of nutrients during the biostimulation treatment. Attempts were made to enhance mineralization of benzene and toluene by changing environmental conditions and nutrient status; however they were unsuccessful except for the addition of acetate. Acetate enhanced the mineralization of toluene in the contaminated core samples. Peroxide was also used for amendment of samples. It was found that for peroxide-amended samples in the biostimulated core, toluene mineralization was substantially greater than for unamended samples. The results of the laboratory investigation performed on the core samples indicate that microbial numbers and activity still remained enhanced for at least 2 years after the biostimulation process had been terminated.

Reference: Thomas, J. M. , Gordy, V. R., Forenza, S., and Ward, C. H. 1990. "Biodegradation of BTEX in Subsurface Materials Contaminated with Gasoline: Granger, Indiana," Wat. Sci., Vol 22, No. 6, pp 53-62. 
13. Jet Fuel Contamination at Eglin Air Force Base*

(Air Force Engineers, November 1986-1988)

Location: Eglin, Air Force Base, FL.

Date and Cause of Contamination: In 1984, damage to vegetation near the Eglin Air Force Base was discovered. Investigation of the problem revealed a jet fuel pipeline leak. The underground pipeline was leaking JP-4 jet fuel at a rate of about $0.3 \mathrm{gal} / \mathrm{min}$.

Extent of Contamination: An estimated 20,000 gallons of jet fuel leaked. The contamination zone encompassed about 4,000 cu yd of soil and a shallow aquifer. Total hydrocarbon levels in the saturated zone were around $200 \mathrm{mg} / \ell$. The jet fuel contamination consisted of aromatic hydrocarbons.

Hydrogeology: The contaminated aquifer was located just below the surface. Sand, clay, and limestone characterized the site. The aquifer was unconfined on top, yet an impermeable clay preventing spreading of the contaminants below the aquifer. The aquifer was around $40 \mathrm{ft}$ deep and had a permeability of $2.3 \times 10^{-8} \mathrm{~cm} / \mathrm{sec}$.

Initial Treatment: Both a passive skimmer recovery method and hand bailing were undertaken to remove free product contaminants from the site. Approximately 7,400 gal of hydrocarbons were removed initially.

Preliminary Laboratory Studies:

Action Taken: Initially, soil and groundwater samples were collected and tested for microbial population, contaminants, and nutrient availability. Chemical analysis, field analysis, and microbial analysis were performed to obtain the required pretesting information.

Results: Hydrogen peroxide stability was checked. First, $290 \mathrm{mg} / \ell$ of peroxide was added to a slurry containing nutrients and groundwater. Approximately 50 percent of the peroxide decomposed within $6 \mathrm{hr}$ of initial exposure. A second addition of peroxide to the sample resulted in only a 5-percent reduction. Results indicated the most efficient means of adding peroxide in the field would be a low initial start-up concentration of peroxide, then gradually building the concentration level to its maximum.

Restore 375 nutrient was used in both the laboratory studies and field demonstration. During the laboratory study, the samples receiving the nutrients displayed evidence of biodegrading capabilities on extracted fractions of the jet fuel. However, there was no discernible difference in the extent or rate of biodegradation as a result of nutrient addition. Thus, the background nutrient levels were sufficient for biodegradation at the site. The nutrient Restore 375 was also tested in the laboratory for sorptive characteristics and permeability of surface material. The chloride in the nutrient traveled fastest, while both ammonia and phosphate were retained quite significantly in the soil. Precipitation of metals in the soil with the nutrient was also tested.

* Also summarized in The Netherlands NIPHEP Report No. 738708006, Case No. U6. 
All of the studies of the nutrients indicated no significant problems should arise when used in the field test.

Field Study:

Action Taken: An in situ bioremediation study was undertaken at the site. The study lasted 576 days. An extraction/injection system was set up to recirculate and enrich the groundwater. Initially, two injection wells were built with screens 3 and $11 \mathrm{ft}$ below the ground. Four recovery wells were built and estimated to operate at a rate of $2.5 \mathrm{gpm}$. The recovery wells pumped groundwater into infiltration galleries; four galleries were initially set up. A spray irrigation system was also used to recirculate the groundwater. Two tanks were set up to receive water from the recovery wells. One tank aerated the water, and the other served as a settling basin. The air supplied to the water in the aeration tank was set at a 25:1 air to water ratio. Nutrients were also injected into the tanks on a batch basis. Although laboratory studies indicated a sufficient supply of background nutrients, other reasons existed for adding excess: to stabilize precipitation of orthophosphate, and to ensure an excess nutrient supply so that oxygen alone was limiting biodegradation. Peroxide was also added to the tanks. A 35-percent solution (Restore 105) was to be injected. An estimated addition rate of $230 \mathrm{mg} / \ell$ of peroxide would be needed to sustain hydrocarbon decomposition.

Microorganisms Used: Indigenous only. A prelaboratory study indicated the presence of hydrocarbon-degrading microbials.

Nutrients Added: Restore 375 was chosen. The nutrient contained by weight 50 percent ammonium chloride, 20 percent disodium phosphate, 17.5 percent trisodium tripolyphosphate, and 17.5 percent monosodium phosphate. Nutrients were added on a batch basis; a total of 7,800 lb were used for the study.

Oxygen Addition: Restore 105, a 35-percent hydrogen peroxide solution, was continually injected into the system.

Problems that Occurred: After the first few weeks, the infiltration galleries capacity dropped to below design levels. Thus, three more galleries were built. The rate of the injection wells also declined because of clogging by iron phosphate precipitates. An attempt to remove the precipitate by washing with 50-percent $\mathrm{HCl}$ solution was attempted and failed. As a result, the maximum pumping rate was around $1 \mathrm{gpm}$. While the spray irrigation process did not clog, there were difficulties with vegetation growth. The problem was overcome by rototilling the soil frequently. Bubbles were seen through the soil which indicated the peroxide was decomposing too rapidly. The increased decomposition rate was credited to microbial produced enzyme catalysts. A phosphate pretreatment of peroxide solution, clean injection water, and peroxide stocking of the tanks were implemented to attempt to expand the peroxide life, yet the attempts failed. Thus, it appears the peroxide had no measurable effect on oxygen levels beyond the immediate injection points.

Results: The microbial counts remained fairly constant throughout the experiment, ranging from $10^{4}$ to $10^{5}$ colony forming units (CFU). Analysis of contamination revealed a slight decrease in hydrocarbon concentrations in the groundwater when compared with the contaminated control. There were no significant changes in hydrocarbon levels in the soil samples. The lack of hydrocarbon degradation indicates that both soil flushing and biodegradation 
were not successful at cleaning up the site. An analysis was conducted to estimate the amount of hydrocarbons reduced by volatilization and that degraded by biostimulation. The results are an estimated $3,600 \mathrm{lb}$ were removed by volatilization, and only $1,400 \mathrm{lb}$ by biodegradation. The combined total indicates only about 30 percent of the hydrocarbons were removed. The results of this study are that simple biodegradation techniques can encounter serious engineering complications; and because of peroxide delivery problems, no real conclusive results could be attained to determine the efficiency of bioremediation.

Reference: Hinchee, R. E., Downey, D. C., Slaughter, J. K., Seby, D. A., Westray, M. S., and Long, G. M. 1989 (Sep). Enhanced Bioreclamation of Jet Fuels: A Full-Scale Test at Eglin AFB FL, Air Force Engineering and Service Center, FL. 
14. Bioremediation Study at Kelly Air Force Base

US Army Engineers, 1985

Location: Kelly Air Force Base, San Antonio, TX.

Date and Cause of Contamination: From 1940-1955, chromium containing electroplating wastes were disposed of at the site. During the early 1960's, the site was used as a chemical evaporation pit for waste solvents and other organic compounds.

Extent of Contamination: Hydrocarbon contamination, electroplating wastes, chlorinated solvents, aerosols, and chlorobenzene polluted the site. Initial contamination levels measured in May of 1985 are: oil and grease around 140 to $535 \mathrm{ppm}$, antimony around 70.7 to $249 \mathrm{ppm}$, chromium around 9.34 to $233 \mathrm{ppm}$, and zinc around 24.9 to $249 \mathrm{ppm}$. The entire contamination site consisted of 1.7 acres.

Hydrogeology: The first $30 \mathrm{ft}$ of subsurface soil consisted of gravels and sands in a silt and clay matrix. The next 30 feet was predominantly clay and marl. A hydropic conductivity ranging from $0.11 \mathrm{ft} /$ day to $9.26 \mathrm{ft} /$ day and low permeability characterized the gravelly clay loams.

\section{Preliminary Laboratory Studies:}

Action Taken: A test pumping well and two monitoring wells were used to take soil and groundwater samples. The samples were measured for contamination levels, biodegradation possibilities, and permeability characteristics.

Results: Laboratory test indicate in situ microbial population is capable of degrading aliphatic hydrocarbons and aromatic compounds under aerobic conditions and chlorinated hydrocarbons under anaerobic conditions. The soil samples were also tested to determine the effect the nutrient (Restore 375X) and peroxide (Restore 105) addition would have on soil permeability. Results indicate a chloride breakthrough first, followed by phosphate, then calcium phosphate precipitates out, and a permeability reduction of soils following nutrient/peroxide addition.

\section{Feasibility Study:}

Action Taken: A circular area, $60 \mathrm{ft}$ in diameter, was designated at which to perform preliminary biodegradation studies. Nine pumping wells and four injection wells were built. Two monitoring wells were also built, one upgradient from the site, the other down gradient. The pumping wells pumped water into a centralized surge tank, then into pipes where nutrients and peroxide were added. The enriched water was then recirculated back to the subsurface by way of the injection wells. A total flow rate of around $1 \mathrm{gal} / \mathrm{min}$ was established to keep the system at a steady state. The study started in May 1985, and ended in February 1986. During the initial 2 weeks of the study, only nutrients were added. Following this time period, $100 \mathrm{ppm}$ of hydrogen peroxide were added. Every 2 weeks the concentration was increased $100 \mathrm{ppm}$, up to a maximum of $500 \mathrm{ppm}$. Samples were collected only twice during the first 3 months, and more frequently from December to February. Groundwater and soil samples were chemically analyzed and measured for $\mathrm{pH}$ and various chemical levels. 
Microorganisms Used: Indigenous only. Laboratory studies indicated oxygen treated indigenous microorganisms successfully and degraded aliphatic, aromatic, and polar hydrocarbons. The chlorinated species investigated were degraded under anaerobic conditions.

Nutrients Added: The nutrient mix added to the groundwater was purchased from the FNC Corporation (Bentone 375K). The nutrients served to: adjust the $\mathrm{pH}$, supply excess phosphate for $\mathrm{H}_{2} \mathrm{O}_{2}$ stabilization, increase emulsification of insoluble inorganics, and fortify the microbial population. During nutrient addition, the average nitrogen source was $812 \mathrm{ppm}$, phosphorous was 1,598 ppm, and chloride was $1,598 \mathrm{ppm}$.

Oxygen Addition: A 35-percent hydrogen peroxide, purchased from FMC Corporation (Bentone 105), was diluted and added to the groundwater. During the first 3 months, the average peroxide level was $1,328 \mathrm{ppm}$. From December on, the level was decreased to $355 \mathrm{ppm}$. Only a sufficient amount of peroxide was added to import saturated zones and localized pockets of hydrocarbons, not the groundwater.

Problems Encountered: The low hydropic conductivities led to a decline in injection rates, insufficient pumping, and noncontinuous circulation rates. A cyclic circulation process was implemented to overcome these difficulties. A white precipitate was found in tanks and wells following nutrient addition. The precipitate was eliminated by increasing the nutrient concentration and switching to a batch method for adding the nutrients to the groundwater. Also, following nutrient and peroxide addition, was a 93-percent drop in groundwater circulation rate, of which only 21 percent was restored.

Results: The sample group collected after the first 3 months of the study indicates no significant change in either total bacteria counts or hydrocarbon degrading bacteria counts. After the process was shut down in February, various observations were made. One, the TCE and PLE levels had reduced from around $4 \mathrm{ppm}$ to $0.96 \mathrm{ppm}$. Another was a strong correlation between laboratory and field investigations. A few examples are: a reduced permeability, metal immobilization from the site, phosphate lay of chloride breakthrough, and a PCE and TCE decline. Other observations from the site include: sufficient circulation of groundwater, a large uptake of ammonia and phosphate salts by the soil, a reduction in the microbial counts for soil samples, and no significant reduction in microorganisms in the groundwater. Conclusion of the laboratory test on the samples indicates successful degradation of chlorinated aliphatics and petroleum hydrocarbons in the same treatment zone. The study performed at the base shows that bioremediation is possible in low permeable aquifers.

Reference: Wetze1, R. S., Durst, C. M., Davidson, D. H., and Sarno, D. J. 1987 (Jul). In Situ Biological Treatment Test at Kelly Air Force Base, Volume II. Field Test Results and Cost Model. Engineering and Services Laboratory, FL.

Wetzel, Roger S., Durst, Connie M., Spooner, Philip A., Ellis, William D., Sarno, Douglas J., Vickers, Brian C., Payne, Jane R., Floyd, Mark S., and Saleem, Zubair A. 1986 (Apr). In Situ Biological Degradation Test at Kelly Air Force Base, Volume I: Site Characteristics, Laboratory Studies and Treatment System Design and Installation, Engineering Services Laboratory, FL. 


\section{Waste Solvents and Fuel Spill at a Laboratory Facility}

Location: Unknown.

Date and Cause of Contamination: Date unknown. Leaking tanks around an excavated area of a laboratory facility was the cause of the contamination.

Extent of Contamination: The contamination level of hydrocarbons was around $22,700 \mathrm{ppb}$. Xylenes, benzene, toluene, ethylbenzene, and $\mathrm{C}_{4}-\mathrm{C}_{12}$ alkanes, ranging in concentrations from about 1,000 to $3,000 \mathrm{ppm}$, composed the hydrocarbon contamination.

Hydrology: Sand and sandy clay made up the subsurface of the site.

Action Taken: Biorestoration, pump-and-treat, and granular activated carbon (GAC) treatment processes were used to clean up the site. A pump-and-treat method was initially used, followed by the biorestoration process. The restoration project consisted of four injection wells and a pump well with a flow rate of 57 to $95 \mathrm{l} / \mathrm{min}$. Both nutrients and hydrogen peroxide were added to the groundwater to enhance biodegradation of the hydrocarbons. After 72 days of biorestoration, the system became clogged, possibly because of silt and degradation of cement that lined the storage tank. Because of the clay, activated charcoal was then used to finish the decontamination at the site.

Microorganisms Used: Indigenous. The total number of bacteria in the water ranged from 300,000 to $410,000 \mathrm{cells} / \mathrm{ml}$. Hydrocarbon degrading bacteria were approximately $5,800 \mathrm{cells} / \ell$ in the groundwater.

Nutrients Added: Nutrient solution consisting of ammonium chloride and sodium phosphate. Nutrients were added on a batch basis.

Oxygen Addition: Dissolved oxygen levels were low, $0.8 \mathrm{ppm}$, initially, so hydrogen peroxide was continually added to the system. Peroxide addition began after a brief period of nutrients-only additions.

Other Treatment Methods: Free product was initially recovered using a sump pump after biorestoration; GAC was used to finish the cleanup.

Results: The pump-and-treat method removed about $200 \mathrm{cu}$ ft of free product. Following 72 days of nutrient and peroxide addition, the bacterial counts increased 130-fold, and the dissolved oxygen increased to $10.5 \mathrm{ppm}$. The total amount of nutrients used were $440 \mathrm{~kg}$ of nitrogen, and $945 \mathrm{l}$ of hydrogen peroxide. About halfway through the restoration process, hydrocarbon levels had dropped to about $580 \mathrm{ppb}$. Following the termination of biodegradation and GAC treatment, the hydrocarbon concentration was below $10 \mathrm{ppb}$ in the tank vault and soil.

Reference: Lee, M. D., Thomas, J. M., Borden, R. C., Bedient, P. B., Wilson, J. T., and Ward, C. H. 1988. "Biorestoration of Aquifers Contaminated with Organic Compounds," CRC Critical Reviews in Environmental Control, Vol 18, No. $1, \mathrm{p} 44$. 
16. Gasoline Spill

Location: Unknown.

Date and Cause of Contamination: Date unknown. Gasoline spilled.

Extent of Contamination: The spill consisted of $303,000 \quad l$ of unleaded gasoline. After recovery of free product, hydrocarbon concentrations in the soil ranged between 300 and $10,000 \mathrm{ppm}$, and between 50 and $60 \mathrm{ppm}$ in the groundwater.

Hydrology: A zone consisting of fine quartz sand with limestone and dolomite grains, along with ferromagnesium minerals was contaminated. Another subsurface zone contaminated consisted of fine quartz sand, yet had large amounts of fires and salt which caused slow groundwater flow.

Action Taken: A free-product recovery method was initially undertaken. After 5 years, a biorestoration process was tested on a section of the spill. The pilot study area consisted of two triangular patterns of monitoring wells and an inner infiltration gallery. Both nutrients and peroxide were added to the subsurface by way of the gallery.

Microorganisms Used: Indigenous.

Nutrients Added: Nutrients were added on a batch basis through the infiltration gallery. The nutrients added were phosphorous and nitrogen.

Oxygen Addition: Hydrogen peroxide was added through the infiltration gallery. Concentrations were slowly increased from $0 \mathrm{ppm}$ up to $500 \mathrm{ppm}$.

Other Treatment Methods: Free product recovery was initially undertaken.

Results: After 5 years of using a free-product recovery method, concentrations of hydrocarbons in the soil and water were still high. The section of the spill used for the biorestoration pilot study contained about $6,100 \pm$ 2,500 (SD) $\mathrm{kg}$ of gasoline. During the study, total bacteria counts were around $10^{6} \mathrm{cells} / \mathrm{ml}$ in the gallery, and $6 \times 10^{3} \mathrm{cells} / \mathrm{ml}$ outside the gallery. One hundred sixty-four days after starting the pilot study, the hydrocarbon level was reduced 65 percent inside the gallery, and 63 percent outside. The hydrocarbon concentration below the water table was reduced from 2,746 to $1,006 \mathrm{ppm}$; at the water table, the levels dropped from 6,087 to 4,058 ppm. Even after biorestoration, significant amounts of hydrocarbons remained absorbed onto the soil. Continued treatment may have improved the cleanup, however.

Reference: Lee, M. D., Thomas, J. M., Borden, R. C., Bedient, P. B., Wilson, J. T., Ward, and C. H. 1988. "Biorestoration of Aquifers Contaminated with Organic Compounds," CRC Critical Reviews in Environmental Control, Vol 18, No. 1, pp 44-45. 


\section{Gasoline Leakage from an Underground Storage Tank}

(FMC)

Location: Unknown.

Description of the site: Residential community. Domestic water wells were contaminated in 12 homes.

Date and Cause of the Contamination: Date unknown. The cause of the contamination was a long-term, low-level loss of gasoline from an underground storage tank at a local service station.

Extent of the Contamination: Approximately 700 to 1,000 gal of gasoline were lost through the slowly leaking underground storage tank. The impacted area covered $87,000 \mathrm{ft}^{2}$ and included the domestic water wells for 12 homes. Close to 99 percent of the gasoline leaked was absorbed by the surrounding soil. An estimated 8 to $10 \mathrm{gal}$ of gasoline were dissolved in the groundwater, resulting in a contaminant concentration of $\approx 15 \mathrm{ppm}$. The concentration of gasoline in the soil was not determined.

Hydrogeology: The site consisted of 6 to $8 \mathrm{ft}$ of red-brown silty loam over fractured shale and silt stone. The depth to the table was 20 to $25 \mathrm{ft}$.

Action Taken: The cleanup process employed a combination of air stripping to control volatile organic compounds and in situ bioremediation to degrade the gasoline in place. Groundwater was circulated at an average rate of $18 \mathrm{gpm}$.

Microorganisms Used: Indigenous only.

Nutrients Added: Nutrients were injected through a gallery located at the original leak.

Oxygen Source: For the first 25 months of operation, air was sparged through six monitoring wells. Next, to further stimulate bacterial activity, "microbial nutrient" (apparently hydrogen peroxide) was used in place of air sparging.

Other Treatment Methods: The pump-and-treat method of circulating groundwater involved the use of air stripping to remove volatile organic compounds.

Results: During the first 20 months of nutrient injection and air sparging, organic levels in the groundwater were reduced 50 to 85 percent. However, after this initial response, further reductions in the contaminant level were not obtained. The cause was identified as reduced indigenous bacterial activity because of an inadequate oxygen supply. Air sparging was discontinued after 25 months of operation and replaced with 10 months of hydrogen peroxide injection, which resulted in increased bacterial stimulation and continued degradation.

Six months after the implementation of peroxide injection, 8 of 12 wells were decontaminated. Hydrocarbon concentrations in groundwater from the other four wells fell to 200 to $1,200 \mathrm{ppb}$.

Reference: Brown, R. A., Morris, R.D., and Brubaker, G. R. 1985 (Nov). Pollution Engineering, p 25. 
18. Gasoline Leakage from a Belowground Storage Tank

(Groundwater Technology, Inc., date unknown)

Location: Believed to be in Oaks, PA.

Description of Site: Residential area. Increased iron and manganese along with hydrocarbon levels were detected in 10 domestic water supply wells.

Date and Cause of Contamination: Date unknown. A belowground storage tank leaked gasoline.

Extent of Contamination: Hydrocarbon contaminants spread approximately $225 \mathrm{ft}$ in a north-south direction, and $325 \mathrm{ft}$ in the east-west direction. Hydrocarbon levels ranged from 0.01 to $15 \mathrm{mg} / \ell$, whereas iron was 0.1 to $6.7 \mathrm{mg} / \ell$ and manganese was 0.2 to $12.0 \mathrm{mg} / \ell$.

Hydrogeology: A 6- to 7-ft red-brown, silt loam covered the surface of the contaminated area. Below this, fractured shale and silt stone lay. The groundwater was located 20 to $25 \mathrm{ft}$ below the surface.

Action Taken: First, the highly contaminated soil around the storage vessels was removed. The excavated area was then used as an infiltration gallery. Water was treated above ground, enriched with nutrients using a batch feed tank, saturated with oxygen, and then reinjected back into the subsurface around the periphery of the hydrocarbon plume. A central pumping well was used to pull water through the contaminated zone, into the gallery for treatment.

Microorganisms Used: Indigenous only.

Nutrients Added: The 12 observation wells and the infiltration gallery were used for nutrient addition ports. The bulk of the nutrients were batch fed to the tank pit area.

Oxygen Source: Air was sparged into the groundwater through the observation wells. After 11 months of air sparging, hydrogen peroxide was added, $100 \mathrm{mg} / \ell$, through the wells and gallery.

Other Treatment Methods: The highly contaminated soil was removed, and the groundwater was treated above ground.

Results: During the 11 months of air sparging, a 50-to 85-percent reduction in organic contaminants resulted. Some of the monitoring we1ls had no contaminants. The use of hydrogen peroxide increased the dissolved oxygen content from 0.5 to $8.0 \mathrm{mg} / \ell$ in the first $24-\mathrm{hr}$ period. The net result is a $70-$ to 80-percent reduction in total hazardous waste within the aquifer. The combined treatment methods proved to be a most applicable and cost-effective technique.

Reference: Taniya, Paul M., and Smith, William. 1986 (Dec). Aquifer Restoration via Accelerated In Situ Biodegradation of Organic Contaminants, Groundwater Technology, Inc., PA. 
19. Aviation Fuel Leak at a Coast Guard Station*

(Traverse Group, March 1988)

Location: Travis City, MI.

Date and Cause of-Contamination: In 1980, contaminated domestic wells were discovered in a residential area in Travis City. Investigation into the contaminated wells revealed a 10,000-gal spill of aviation fuel. The spill occurred in 1969 at a Coast Guard Station.

Extent of Contamination: The major contaminants were benzene, toluene, ethylbenzene, and xylene (BTEX). The highest level of contamination, found at around $13 \mathrm{ft}$ below the surface, was about $5,590 \mathrm{mg} / \mathrm{kg}$ wet solids. The spill site was about 50 to $120 \mathrm{~m}$ wide and $1,300 \mathrm{~m}$ long.

Hydrogeology: The subsurface was mostly sand and gravel. The groundwater, found about $5 \mathrm{~m}$ below the surface, had a velocity ranging from 1 to $2 \mathrm{~m}$ per day. Hydropic conductivity was measured to be between 28 and $50 \mathrm{~m}$ per day.

Action Taken: An extensive monitoring project was undertaken prior to site cleanup. Nine monitoring wells and 12 cluster wells were used to gather soil samples from depths ranging between 13 and $25 \mathrm{ft}$. The data collected was used to determine the extent of contamination and an approximate cleanup design. A two-dimensional model, BIOPLUME II, was used to design the biorestoration project. The BIOPLUME II was developed at Rice University to simulate hydrocarbon transport under the influence of biodegradation.

A pilot study was undertaken to investigate the use of bioremediation and also access the use of BIOPLUME II. Ten injection wells were built. Clean groundwater was pumped from a well $600 \mathrm{ft}$ from the site into the injection wells.

Clean groundwater was pumped into five injection wells which had screens at 25 and $29 \mathrm{ft}$ at a rate of $29 \mathrm{gpm}$. Water enriched with nutrients and either oxygen or peroxide was injected into the five remaining wells which had screens at $14 \mathrm{ft}$ and $19 \mathrm{ft}$ at a rate of $11 \mathrm{gpm}$. The study began in March 1988 when oxygen was added to acclimate the system. Nutrient addition began in May 1988, and in June 1988, hydrogen peroxide was used instead of oxygen.

Microorganisms Used: Indigenous.

Nutrients: Nutrients were first diluted with clean water, then injected into the subsurface at a rate of $11 \mathrm{GPM}$. The nutrient levels were about $140 \mathrm{mg} / \mathrm{l}$ of ammonia and $100 \mathrm{mg} / \mathrm{l}$ of phosphate.

Oxygen Addition: Pure oxygen was originally added to the subsurface at about $40 \mathrm{mg} / \ell$. After 3 months from initial start-up, hydrogen peroxide was used instead of oxygen. The first week of peroxide addition, the level was $50 \mathrm{mg} / \mathrm{l}$. The second week it was $100 \mathrm{mg} / \mathrm{l}$, the following week $250 \mathrm{mg} / \mathrm{l}$. The peroxide level was kept at $250 \mathrm{mg} / \ell$ for 9 more weeks, from up which the level was increased to $500 \mathrm{mg} / \ell$ for another 9 weeks.

* Also summarized in the Netherlands NIPHEP Report No. 738708006, Case No. U3. 
Results: Oxygen breakthrough occurred at the $31 \mathrm{ft}$ monitoring well 1 month from start-up, and at the 50-ft well it took 2 months. The BTEX concentration began to decrease 5 days after system start-up. At the $31-\mathrm{ft}$ well, BTEX levels had declined to an acceptable limit after 6 months. At the 50-ft well, BTEX concentrations were around $530 \mathrm{mg} / \ell$, and after 7 months, had dropped to $50 \mathrm{mg} / \ell$. The elevation in dissolved oxygen levels corresponded to the decrease in contaminants. The pilot study was successful for both degrading contaminants and also matching BIOPLUME II's predict results with an actual field test. The test showed that oxygen and nutrients were consumed, and contamination levels dropped. The entire site had not been completely cleaned up as of January 1990, yet bioremediation was occurring.

References: Ward, C. H., Thomas, J. M., Florenza, S., Rifai, H. S., and Bedient, P. B. 1989. "In Situ Bioremediation of Subsurface Material and Ground Water Contaminated with Aviation Fuel: Traverse City, Michigan," Proceedings of the 1989 A \& WMA/EPA International Symposium, pp 83-96.

Ward, C. H., Thomas, J. M., Florenza, S., Rifai, H. S., and Bedient, P. B. 1988 (Nov). "A Quantitative Demonstration of the Raymond Process for In Situ Biorestoration of Contaminated Aquifers," Proceedings of Petroleum Hydrocarbons and Organic Chemicals in Ground Water. Prevention. Detection and Restoration, Vol II, Houston, pp 723-743.

Rifai, Hanadi S., Bedient, Philip B., Wilson, John T., Miller, Karen M., and Armstrong, John M. 1988 (Oct). "Biodegradation Modeling at Aviation Fuel Spill Site," Journal of Environmental Engineering, Vol 114, No. 5, pp 1007-1029.

Thomas, J. M., and Ward, C. H. 1990 (Mar). "Bioremediation of Contaminated Aquifers," The Third Annual Hazardous Materials Management Conference/Central, pp 388-393.

Armstrong, John M. 1989 (Jan). National Demonstration Sites: In-Situ Bioremediation of Contaminated Aquifer, The Traverse Group, Inc.

Rifai, Hanadi S., and Bedient, P. B. 1987 (Nov). "BIOPLUME II-Two Dimensional Modeling for Hydrocarbon Biodegradation and In Situ Restoration," Proceedings of: Petroleum Hydrocarbons and Organic Chemicals in Ground Water: Prevention. Detection and Restoration, pp 431-439. 
20. Spillage of Organic Chemicals Near a Shallow Aquifer

(OH Materials, 1980)

Location: Unknown.

Description of the Site: Grazing land.

Date and Cause of Contamination: Date unknown. Accidental leakage from storage vessels resulted in a large organic chemical spill.

Extent of Contamination: One hundred and thirty thousand gallons of organic chemicals were spilled. Groundwater contamination levels were as high as $10,000 \mathrm{ppm}$ of organic chemicals. The spill penetrated through a permeable vallose zone into a shallow aquifer, about $15 \mathrm{ft}$ thick, directly below the site.

Action Taken: Two types of processes were used: a recovery injection process and biodegradation. The recovery injection process entailed pressure injections to flush contaminants and recovery of groundwater. Recovered groundwater was decontaminated by a filtration method involving activated carbon. The air was also purified using carbon filtration. Monitoring wells were installed in the recovery area so that cleanup progress could be measured. Samples were taken from the wells at $1.5,3,6,9$, and $12 \mathrm{ft}$. Once the contaminants were below $1,000 \mathrm{ppm}$, the biological treatment was started.

Water, previously treated, was enriched with nutrients and hydrocarbon degrading bacteria to initialize biodegradation. The enriched water was reinjected back into contaminated zone where the dissolved oxygen concentrations were maintained.

Hydrogeology: The contaminated aquifer is $15 \mathrm{ft}$ thick and located under a permeable vadose zone. The rate of groundwater trave1 is around $0.8 \mathrm{ft} /$ day. Fifty to sixty feet of silty clay underlay the aquifer.

Microorganisms used: Indigenous and also added hydrocarbon degrading bacteria were used. The bacteria was added to treated water prior to reinjection.

Nutrients Added: Nutrients were added to previously treated groundwater prior to reinjection back into the aquifer.

Oxygen Source: A high dissolved oxygen level was maintained. The method used was not described.

Other Treatment: The groundwater was treated above ground initially by filtering contaminants through activated carbon.

Results: The recovery/injection process resulted in a decrease of organic chemicals to around $1,000 \mathrm{ppm}$. The biodegradation process, in approximately 2 months, reduced the chemical concentration to around $150 \mathrm{mg} / \mathrm{l}$. Post sampling indicated the chemical contamination was below $1 \mathrm{ppm}$, thus completing the cleanup.

Reference: Ahrech, Robert J., and Gardner, Gary L. 1982. "Restoration of an Aquifer Contaminated by an Accidental Spill of Organic Chemicals," GWMR, pp 50-53. 


\section{Clean up of a Methylene Chloride Spill}

Extent of Contamination: The concentration of methylene chloride in the groundwater was $192 \mathrm{ppm}$ in samples taken from the monitoring wells. The source wells had an initial methylene chloride concentration of $20,000 \mathrm{ppm}$.

Action Taken: Both aboveground and in situ biorestoration treatments were used. Sand filtration, air stripping, and a pump-and-treat method for the groundwater were implemented. The treated water was amended with nutrients and bacteria, then used to flush the contaminated soil.

Microorganisms Used: Organisms from a wastewater treatment plant receiving methylene chloride were used.

Nutrients Added: Ammonia and phosphate were added to contaminated soil.

Other Treatment Methods: Both physical and biological aboveground treatments were used. Air stripping, filtration, and an activated sludge unit were used to decontaminate the groundwater.

Results: Air stripping reduced approximately 99 percent of the contaminants in the water. After 43 days from the start of the biorestoration process, the methylene chloride was reduced to $6 \mathrm{ppm}$, and $156 \mathrm{ppm}$ chloride was released. The methylene chloride in the source wells had reduced to less than $1 \mathrm{ppm}$.

Reference: Lee, M. D., Thomas, J. M., Borden, R. C., Bedient, P. B., Wilson, J. L., and Ward, C.H. 1988. "Biorestoration of Aquifers Contaminated with Organic Compounds," CRC Critical Reviews in Environmental Control, Vol 18, No. $1, \mathrm{p} 40$. 
22. Leak in Underground Piping at Biocraft Labs

(Groundwater Decontamination System)

Location: Waldwick, NJ .

Description of Site: Industrial park.

Date and Cause of Contamination: In August 1975, contamination was observed in a small creek. The source of the contamination was an underground leak in a piping system used to transport solvents.

Extent of Contamination: Twelve hundred cubic yards of soil in a 1.75-acre area was contaminated. Approximate amounts of contamination were 18,150 lb of methylene chloride, 66,825 $\mathrm{lb}$ of N-butyl alcohol, 26,300 lb of dimethylanilene, and 10,890 $\mathrm{lb}$ of acetone.

Hydrogeology: Specific details not given.

Action taken: Three trenches were used to recirculate the contaminated plume. A pipe collection trench, located down gradient from the contaminated area, collected water. The water was then treated above ground using an aerobic biological process, then reinjected upgradient from the spill through the other two trenches. In situ biodegradation was enhanced by injecting air through equally spaced wells into the subsurface contaminated groundwater. Twenty-two monitoring wells were used to continuously record the groundwater activities.

Microorganisms Used: No information given.

Nutrients Added: No information given.

Oxygen Source: Wells supplied oxygen to the groundwater.

other Treatment: The contaminated plume was treated above ground.

Results: Approximately 60 percent of the contamination removal occurs above ground, and the remaining 40 percent below ground,. The removal efficiency for methylene chloride, an N-butyl alcohol, using aerobic treatment, is 98 percent. Removal efficiency is 97 percent for acetone and 93 percent for dimethylaniline. An estimated 5 years will be needed to decontaminate the site. Conclusive results were not given.

Reference: Amdurer, Michael, Fellman, Robert, and Abdelhamid, Salah. 1985 (Sep). "In Situ Treatment Technologies and Superfund," International Conference on New Frontiers for Hazardous Waste Management, Environmental Protection Agency, pp 415-425. 
23. Spillage of Four Different Solvents

Location: New Jersey

Date and Cause of Contamination: Date unknown. Four solvents-methylene chloride, N-butanol, acetone, and dimethylaniline-were spilled into a glacial till aquifer.

Extent of Contamination: The concentration of the solvents in the aquifer were $19.1 \mathrm{mg} / \ell$ for $\mathrm{N}$-butanol, $58.5 \mathrm{mg} / \ell$ for methylene chloride, $2.9 \mathrm{mg} / \ell$ for dimethylaniline, and $38.8 \mathrm{mg} / \ell$ for acetone.

Action Taken: A pump-and-treat method was used to clean up the groundwater. Once treated, the groundwater was aerated and enriched in nutrients, then recirculated back into the sublayer. Oxygen was supplied to the aquifer through a series of injection wells.

Microorganisms Used: Organisms acclimated to the solvents were added to treated groundwater.

Nutrients Added: Nutrients were added to the treated groundwater. The nutrients were nitrogen, phosphate, magnesium, sulfate, carbonate, manganese, and iron.

Oxygen Added: Oxygen was added through a series of injection wells.

Other Treatment Methods: The groundwater was treated above ground.

Results: Aboveground treatment removed 97 percent of the methylene chloride, $\mathrm{N}$-butanol, and acetone, and 93 percent of the dimethylaniline from the groundwater. The solvents in the effluent were decreased to: $0.04 \mathrm{mg} / \ell$ of $\mathrm{N}$-butanol, $0.92 \mathrm{mg} / \ell$ of methylene chloride, $0.18 \mathrm{mg} / \ell$ of dimethylaniline, and $1.12 \mathrm{mg} / \ell$ of acetone. After 3 years of treatment, the plume was reduced in size by 90 percent, the chemical oxygen demand was reduced from 300 to $20 \mathrm{mg} / \ell$, yet solvents were still detected in the groundwater. Projected cleanup was to be completed within the 3-year period; however, New Jersey Department of Environmental Protection standards had not been achieved at the 3-year mark.

References: Lee, M. D., Thomas, J. M., Borden, R. C., Bedient, P. B., Wilson, J. T., and Ward, C. H. 1988. "Biorestoration of Aquifers Contaminated with Organic Compounds," CRC Critical Reviews in Environmental Control, Vol 18, No. 1, pp 34-35. 
24. Ethylene Glycol Contamination at the Naval Air Engineering Center in Lakehurst, New Jersey

Location: Lakehurst, NJ .

Date and Cause of Contamination: Date unknown. Cooling water leaked from a storage lagoon at the Naval Air Engineering Center in Lakehurst.

Extent of Contamination: Approximately 4,000 gal of cooling water spilled. The soil contamination level was around $4,960 \mathrm{mg} / \mathrm{kg}$ of soil, while the groundwater was around $2,100 \mathrm{mg} / \ell$.

Action Taken: The groundwater was treated above ground in an activated sludge unit. The treated water was enriched with bacteria, nutrients, and oxygen, then reinjected into the subsurface through wells. After termination of the biorestoration process, lime and diammonium phosphate were added to the soil surface.

Microorganisms Used: Indigenous and ethylene glycol-degrading bacteria were used.

Nutrients Added: Nitrogen and phosphorous were added to treated groundwater, then reinjected back into the contaminated soil using injection wells.

Oxygen Source: Not specified; however, treated water was amended with oxygen.

Other Treatment Methods: The groundwater was treated above ground. A final passive treatment, after biorestoration, was implemented. The treatment consisted of adding lime and diammonium phosphate to the soil.

Results: After 26 days, the ethylene glycol level in the groundwater had reduced to less than $50 \mathrm{ppm}$ from around an initial $550 \mathrm{ppm}$. However, the unsaturated zone still contained pockets of ethylene glycol. After both the biorestoration and passive processes were terminated, ethylene glycol could not be detected in collected groundwater samples.

References: Lee, M. D., Thomas, J. M., Borden, R. C., Bedient, P. B., Wilson, J. T., and Ward, C. H. 1988. "Biorestoration of Aquifers Contaminated with Organic Compounds," CRC Critical Reviews in Environmental Control, Vol 18, No. 1 , pp 40-41. 
25. Lumber Mill Pilot Study Involving Biodegradation to Remove WoodTreating Chemicals

(Woodward-Clyde Consultants, 1987)

Location: Libby, MT.

Description of Site: A lumber mill.

Date and Cause of Contamination: Residents drilling irrigation wells first detected the contamination in the late 1970's. The spill of pentachlorophenol (PCP) and creosote occurred between 1946 and 1969.

Extent of Contamination: The soil and groundwater were contaminated by the wastes, sludge, and spillage of the PCP and creosote. Three monitoring wells were set up, a control well and two others labeled A and B. The control well had a PCP concentration of $200 \mathrm{mg} / \ell$, A well had a concentration of $1,500 \mathrm{mg} / \ell$, and $B$ well was at $2,250 \mathrm{mg} / \ell$.

Action Taken: A pilot study was undertaken to investigate the possibility of using bioremediation to clean up the spill site. Five wells were used in the study: 2 injection wells located within the plume, wells A and B placed down gradient from the injection wells, and a control well placed upgradient from the injection wells. The injection wells were used to supply nutrients and hydrogen peroxide to the indigenous microorganisms.

Hydrogeology: Material around the lumber mill ranged from clays to cobbles and were distributed in a mosaic fashion. Two aquifers, one 15 to $70 \mathrm{ft}$ below the surface, the other 100 to $105 \mathrm{ft}$ below the surface, were in the contaminated area.

Microorganisms Used: Indigenous only.

Nutrients Added: Nutrients were added beginning approximately in January of 1988 on a monthly basis. Nutrients addition began 7 months prior to the peroxide injections.

Oxygen Source: $100 \mathrm{mg} / \ell$ of peroxide was added 15 and $40 \mathrm{ft}$ below the surface at a rate of $20 \mathrm{gpm}$.

Other Treatment Methods: None for the pilot study.

Results: The natural dissolved oxygen levels at the start of the study ranged between 1 and $5 \mathrm{mg} / \ell$ in wells $\mathrm{A}, \mathrm{B}$, and the control well. After 148 days of peroxide addition, samples from the control well and well $B$, which was located furthest downgradient from the injection wells, had not changed significantly. Samples collected from well A had oxygen concentrations of 15 to $20 \mathrm{mg} / \ell$.

Sixteen months after the peroxide addition began, well A had an oxygen concentration of $1 \mathrm{mg} / \ell$, well B of 0.3 to $1.0 \mathrm{mg} / \ell$, and the control well was 0.4 to $2.5 \mathrm{mg} / \ell$. The decrease in dissolved oxygen indicates a reduction in organic contaminants.

Measurements of contaminants indicated a modest reduction in levels for well B. For well A, a below detectable limit after 125 days of the initial peroxide addition was reported. The dissolved contamination levels in the pilot study area remained high, with exception of well A. Thus, the large volumes 
of groundwater flowing through the area failed to significantly remove absorbed contamination.

Data from the study supports using a biodegradation process to clean up the spill. Evidence that the process will work includes changes in dissolved oxygen level, variable rates of nutrient removal and transformation, changes in microbial content, and a reduction in contaminants around well $\mathrm{A}$.

References: Doyle, Robert, and Piotrowski, Michael. 1989. "In Situ Bioremediation of Wood-Treating Compounds in Groundwater," 1989 RCRA/Superfund Conference, pp 308-314.

Piotrowski, Michael R. 1989 (Aug). "Bioremediation: Testing the Waters," Civil Engineering, pp 51-53. 
26. Contamination of a Shallow Aquifer in the Southeastern United States (Martin A. Rowland, 1984)

Location: Southeastern United States

Description of Site:- Storage facility in a plant.

Date and Cause of Contamination: A large spill of trichloroethylene (TCE) occurred in 1966. In addition, small leakage of TCE had been occurring for over a 20-year period.

Extent of Contamination: The contamination area is around $500 \mathrm{ft}$ by $500 \mathrm{ft}$. TCE concentrations in two aquifers, one at $20 \mathrm{ft}$ depth, the other at $40 \mathrm{ft}$, ranged from 11,000 to $670,000 \mathrm{mg} / \ell$ during the period from 1984 to 1988 . The solubility of TCE in water is around $1,200 \mathrm{mg} / \ell$.

Action Taken: Measurements were taken during 1984 to 1988 of TCE and two biodegradation byproducts: 1,2-dichloroethylene (DCE) and vinyl chloride (VC). Eight groundwater monitoring wells were used to facilitate sample collection and groundwater monitoring. Two of the eight wells were used to remove contaminated groundwater for treatment.

Microorganisms used: Indigenous only.

Nutrients Added: None.

Other Treatment Methods: Wells were monitored to determine the biodegradation extent of TCE. A comparison of the rate of TCE degradation from the measurements to a separate field study and a rate determined under laboratory conditions was performed. Both field studies were in agreement; however, these rates were slightly slower than the laboratory study. The field studies showed a 50-percent degradation of TCE in 36 weeks, whereas the laboratory's results indicated a 40-percent degradation of TCE in 8 weeks. The difference in rates can be attributed to environmental conditions. Thus, biodegradation was occurring at the site where TCE concentrations were low, the hundreds of $\mathrm{mg} / \ell$ range.

Reference: Rowland, Martin A., and Eisenbury, Tolbert N. 1989. "Anaerobic Biodegradation of Trichloroethylene in a Shallow Aquifer, " RCRA/Superfund Conference, pp 188-191. 
27. Formaldehyde Spill near Ukiah, California

(IT Corporation)

Location: Near Ukiah, CA.

Description of Site: Railroad station located near drainage and irrigation ditches.

Date and Cause of Contamination: On March 25, 1982, a vandalized railroad car leaked formaldehyde.

Extent of Contamination: Approximately 20,000 gal of a 50-percent formaldehyde solution leaked from a tank car into a drainage system. Because of heavy rains, a large runoff of diluted formaldehyde occurred. The runoff consisted of approximately three million gallons of contaminated water with a formaldehyde concentration ranging from 1,000 to $5,000 \mathrm{ppm}$. The contaminated soil, located as far as $100 \mathrm{ft}$ from the spill, had a formaldehyde concentration ranging from 20,000 to $50,000 \mathrm{ppm}$.

Hydrogeology: Specific details not given.

Action Taken: To prevent spreading of the formaldehyde, drainage ditches were dammed and the water pumped out and hauled away. The contaminated water was treated at a disposal facility in Martinez, CA, using an oxidation process.

The contaminated soil was treated both chemically and through biodegradation. Oxidation of the formaldehyde by chemicals was first performed. Initially, sodium hydroxide and soda ash were added to the site, then an alkaline solution and a 5-percent hydrogen peroxide solution were alternately sprayed onto the site. Approximately 6,000 gal of alkaline solution and 12,000 gal of 5 percent peroxide were used. After 2 days of chemical treatment, the biological process was implemented.

The biological process consisted of spraying chemicals and inoculum periodically onto the contaminated site. Liquid materials at the site were recirculated by using an excavated ballast as a sump, pumping materials from the sump, through an aeration tank, onto the contaminated soil, and then collecting the runoff back into the sump. After stopping the process, peroxide was sprayed one last time onto the site.

Microorganisms Used: An inoculum, Hydrobac from Polyboc, Inc., was sprayed periodically onto the site.

Nutrients Added: Along with the inoculum, chemicals were added to the site.

Oxygen Source: The recirculated liquid was first aerated before being sprayed onto the site.

Other Treatment: The contaminated water was treated at a facility located approximately 100 miles from the site. Prior to and after biodegradation, the formaldehyde was oxidized using hydrogen peroxide.

Results: The formaldehyde concentration in the contaminated water was brought down to below $1 \mathrm{ppm}$ at the Martinez plant. Initial peroxide treatments on the contaminated soil resulted in a formaldehyde concentration of 5,000 ppm after 
4 to $6 \mathrm{hr}$. Following 2 days of treatment, concentrations were ranging from 500 to $1,000 \mathrm{ppm}$.

The biological process resulted in a further drop of the formaldehyde concentration. After a 5-day acclimation period, and 17 days more, the formaldehyde concentration was below $1 \mathrm{ppm}$. After this, the final peroxide treatment was performed, which resulted in a final formaldehyde concentration of less than $42 \mathrm{ppb}$. The spill was then considered detoxified and the cleanup process complete.

Reference: Spikes, D. J., McCullock, M. N., and Blackburn, J. W. 1984. "The Containment and Mitigation of a Formaldehyde Rail Car Spill Using Novel Chemical and Biological In-Situ Treatment Techniques," 1984 Hazardous Material Spill Conference, pp 38-44. 
APPENDIX B:

LIST OF ATTENDEES TO THE ADVISORY GROUP MEETING AUGUST 1990 


\section{ATTENDEES LIST FOR IN SITU BIOTREATMENT STUDY \\ TECHNICAL WORKING GROUP \\ US ARMY ENGINEER WATERWAYS EXPERIMENT STATION}

AUGUST 14 AND 15, 1990

Working Group Members:

Dr. Martin Alexander

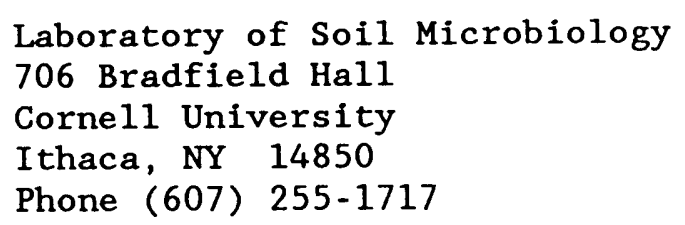

Dr. Perry McCarty

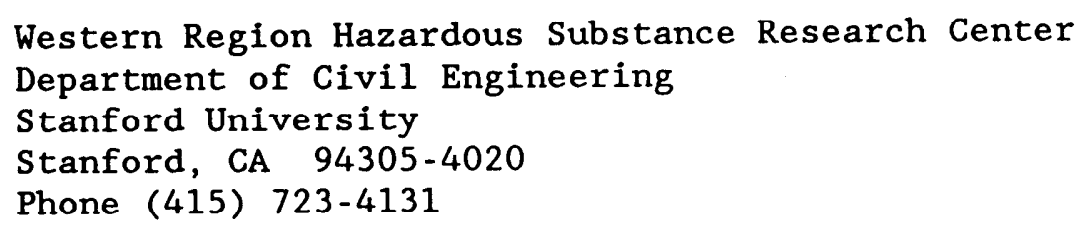

Dr. John Wilson

Technical Support Division

Robert S. Kerr Environmental Research Laboratory

Box 1198

Ada, OK 74820

Phone (405) 332-8800, Ext. 256

Dr. James Spaine

USAF Tyndall AFB

AFESC/RDVC

Tynda11, FL 32403

Phone (904) 283-2982

Dr. C. H. Ward

Department of Environmental Science and Engineering Rice University 6100 South Main

ML 102

Houston, TX 77005

Phone (713) 527-4086

WES Attendees:

USACE -WES

3909 Halls Ferry Road

Vicksburg, MS 39180 
Mr. Norman Francingues

Environmental Laboratory

Environmental Engineering Division

Phone (601) 634-3703

Mr. Mark Zappi

Environmental Laboratory

Environmental Engineering Division

Phone (601) 634-2856

Dr. Doug Gunnison

Environmental Laboratory

Ecosystem Research and Simulation Division

Phone (601) 634-3873

Dr. Judy Pennington

Environmental Laboratory

Ecosystem Research and Simulation Division

Phone (601) 634-2802

Dr. John Cullinane

Environmental Laboratory

Environmental Engineering Division

Phone (601) 634-3723

Ms. Cindy Teeter

Environmental Laboratory

Environmental Engineering Division

Phone (601) 634-4018

Dr. James May

Geotechnical Laboratory

Earthquake Engineering and Geophysics Division

Phone (601) 634-3395

Mr. Tommy Myers

Environmental Laboratory

Environmental Engineering Division

Phone (601) 634-3939

Dr. James Brannon

Environmental Laboratory

Ecosystem Research and Simulation Division

Phone (601) 634-3725 
Dr, Carlos Ruiz

Environmental Laboratory

Ecosystem Research and Simulation Division

Phone (601) 634-3784

Dr. Stafford Cooper

Geotechnical Laboratory

Earthquake Engineering and Geophysics Division

Phone (601) 634-2477

Dr. Jerry Pabst

Environmental Laboratory

Ecosystem Research and Simulation Division

Contractor

Phone (601) 634-2435

Non-WES Attendees:

Dr. Fran Kramer

EPA

401 M Street SW

os 110

Washington, DC 20460

Phone (202) 475-6647

Dr. Shanka Banerji

Dept. of Civil Engineering

University of Missouri-Columbia

107 Engineering

Columbia, MO 65211

Phone (314) 882-6269

Dr. Robert Sproul1

Department of Chemical Engineering

Oregon State University

Corvallis, OR 97331-2702

Phone (503) 737-2408

CPT Craig Myler

USATHAMA

US Army Toxic and Hazardous Materials Agency

Attn: CETHA-TS-D

Aberdeen Proving Ground, MD 21010-5401

Phone (301) 671-2054 
Mr. Wayne Sisk

USATHAMA

US Army Toxic and Hazardous Materials Agency

Attn: CETHA-TS-D

Aberdeen Proving Ground, MD 21010-5401

Phone (301) 671-2054 
Work is underway at the US Army Engineer Waterways Experiment Station (WES) in Vicksburg, MS, in support of the Army's research on toxic and hazardous waste. This work is part of the Army's Installation Restoration Research program, which is managed by the US Army Corps of Engineer Toxic and Hazardous Materials Agency (USATHAMA) at Aberdeen Proving Ground, Maryland. Recent advances in the ability of microorganisms to degrade toxic chemicals in situ in soils looks promising to the Army researchers; therefore, USATHAMA has tasked WES to develop in situ biological treatment as another technology to assist in the Army's efforts to clean up toxic waste at chemical and munitions plants across the nation. To further the development and field implementation of in situ biological treatment, a group of experts met recently for critical review of the WES research. Attendees included representatives from the Army, Air Force, US Environmental Protection Agency (EPA), and academia. The group endorsed the overall plan of development outlined and agreed that the technical approach presented by WES researchers appeared to be scientifically and procedurally strong. Future meetings of experts and specialists in field implementation of systems will be scheduled as results of the research become available. 


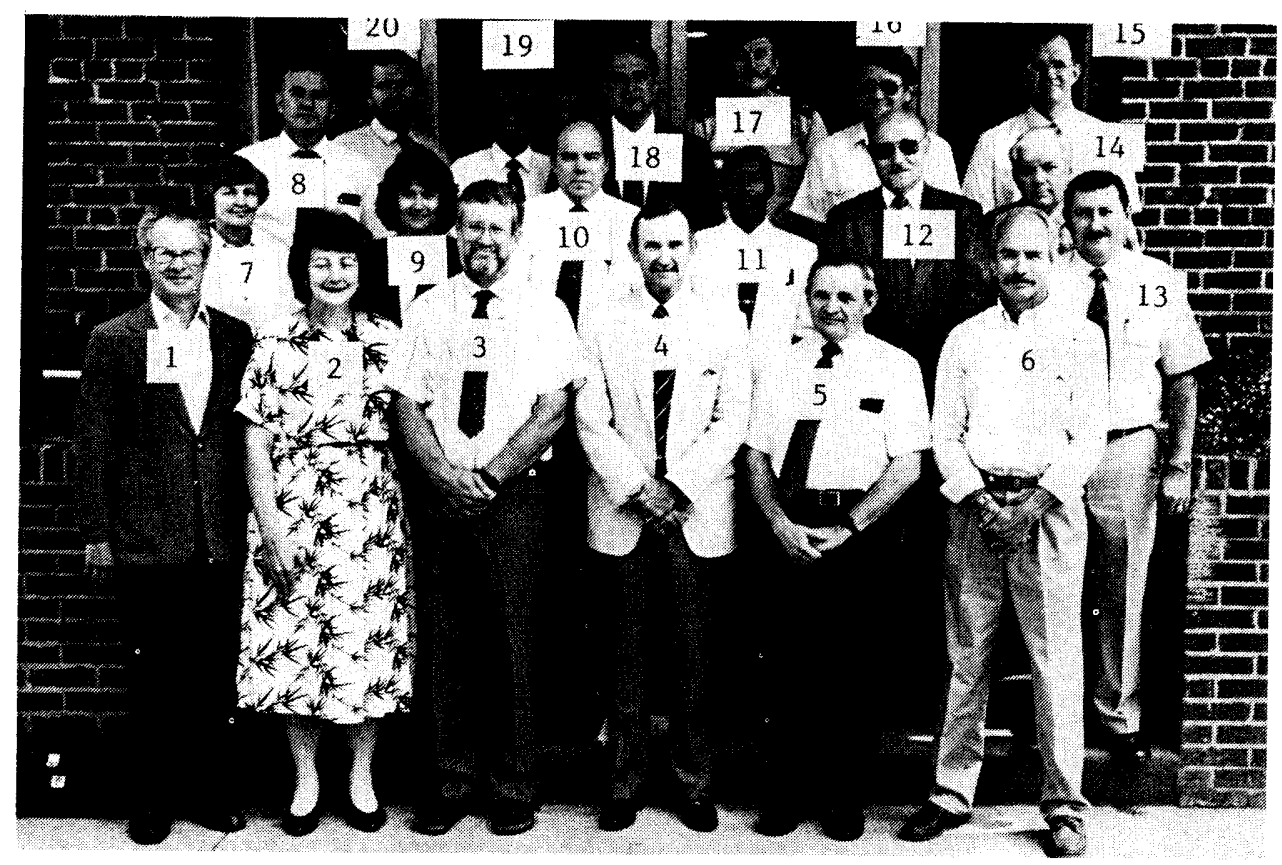

(1) Dr. Perry McCarty

Stanford University

(2) Dr. Fran Kramer EPA

(3) Dr. John Wilson

Robert S. Kerr Environmental Research Laboratory

(4) Dr. Herbert Ward Rice University

(5) Dr. Martin Alexander Cornell University

(6) Dr. James Spaine US Air Force

(7) Dr. Judy Pennington WES

(8) Mr. Wayne Sisk USATHAMA

(9) Ms. Cindy Teeter WES

(10) Dr. John Cullinane WES
(11) Mr. Roy Wade

WES

(12) Dr. Doug Gunnison

WES

(13) Mr. Norman Francingues WES

(14) Dr. Jerry Pabst WES

(15) Dr. Jim Brannon WES

(16) Mr. Tom Myers WES

(17) Capt. Craig Myler USATHAMA

(18) Mr. Mark Zappi WES

(19) Dr. Shanka Banerji University of Missouri Columbia

(20) Dr. Robert Sproull Oregon State University 
APPENDIX $C$ :

SUMMARY OF ADVISORY COMMITTEE MEETING AUGUST 1990 


\section{MEMORANDUM FOR RECORD}

SUBJECT: Meeting of the In situ Biological Treatment Technical Working Group

1. A meeting of the Technical Working Group on the In Situ Biological Treatment Program (Project AF25) was held from Tuesday, August 14 through Wednes day, August 15, 1990, at the US Army Engineer Waterways Experiment Station (WES). Members of the Working Group are: Dr. Martin Alexander, Cornell University; Dr. Perry McCarty, Stanford University; Dr. John Wilson, Robert S. Kerr (RSK) Environmental Research Laboratory (USEPA); Dr. James Spaine, USAF Tyndall Air Force Base; and Dr. C. Herbert Ward, Rice University. Addresses and phone numbers of other attendees are listed in Appendix B.

2. Mr. Mark Zappi of the Environmental Engineering Division (EED), WES, opened the meeting by introducing Dr. John Harrison, Chief of the Environmental Laboratory (EL), who presented welcoming remarks on behalf of WES. This was followed by the introduction of attendees by Mr. Zappi. CPT Craig Myler, of the US Army Toxic and Hazardous Materials Agency (USATHAMA), presented a brief description of the historical development of hazardous waste cleanup programs and goals of USATHAMA. In the area of explosive remediation, the past technologies have concentrated on capping, incineration, and composting. By 1995 , USATHAMA projects that involve chemical/physical technologies will be implemented at a cost of approximately $\$ 30$ to $\$ 40$ a ton. In addition, bioremediation will be pursued as a means of treating explosive contaminated soils. Remediation of solvent contaminated soils from 1975 to 1990 have been dig and haul, in-place volatilization, and thermal stripping. By 1995, USATHAMA projects that involve in situ biotreatment of solvent contaminated soils will be implemented at a cost of $\$ 40$ to $\$ 60$ a ton. From 1975 to 1990 , the technologies for the remediation of groundwater have concentrated on no treatment, air stripping (with controlled emissions), carbon adsorption, ion exchange, and pump-and-treat technologies. It is hoped that in situ treatment technologies will be a treatment option for groundwater by 1995 . Some discussion then followed about Rocky Mountain Arsenal and its relation to problems of military significance and the role of USATHAMA and its status within the Corps of Engineers.

3. The Working Group members had the following questions regarding the research program proposal: (a) funding for this research program, (b) level of research to be pursued, and (c) duration, scheduling, and implementation of the research program. Mr. Wayne Sisk, USATHAMA, indicated that $\$ 3$ to 5 millon is planned for in situ biodegradation over the next 5 to 6 years.

4. The working portion of the meeting opened with an overview presented by $\mathrm{Mr}$. Zappi on the in situ program and its objectives. The program objectives are to develop in situ technologies based on biological processes that can be used to remediate contaminated sites at Department of Army installations. The objectives of the working group meeting were to:

(a) Identify deficiencies in the proposed technical approach for the bench studies; 
(b) Identify strong points of the proposed technical approach;

(c) Obtain input and advice from the Working Group; and

(d) Provide a forum for "technical brainstorming."

Mr. Zappi then outlined the progression of the research program as follows:

(a) Identify technology knowledge gaps;

(b) Develop the technical approach and conduct research from the bench scale;

(c) Develop technical approach, conduct research, and obtain results from the pilot scale; and

(d) Conduct field evaluations of various biological processes at the contaminated sites.

5. Following Mr. Zappi's presentation, a lengthy discussion was initiated by Dr. McCarty who expressed his concerns that the objectives of the research program are too broad and should be more focused. Dr. Alexander felt that much of the work is already being pursued and is highly chemical specific. suggested WES determine, for any given chemical contaminant, what is known about the chemical, what sites are contaminated with it, and then pursue research using soils from those sites all the way through to a field demonstration. Dr. Alexander suggested that 5 contaminated soils and 5 classes of compounds made 25 issues for each part of the research program. He suggested that WES focus on reducing the number of media or the number of contaminants, or both, i.e., concentrate on army-specific compounds (e.g., TNT, RDX). This reduction in scope would reduce the number of media and chemicals that have to be examined and allow real depth study. Dr. Ward agreed with Dr. Alexander's comments and stated that the technology approach drafted by WES was a good first effort, but that WES could not conduct the proposed program for $\$ 3$ to $\$ 5$ million. Dr. Wilson suggested that WES use a $5 \times 5$ soil and chemical group matrix to identify the areas of concern and eliminate areas being covered by other research groups.

6. The topic of discussion then changed to concerns with financial and research efficiency. CPT Myler asked Dr. Kramer (EPA Biosystems Development Technologies Program) about funding levels for EPA research programs and the efforts EPA was supporting with its funds. Dr. Kramer indicated that in keeping with the mission of the EPA Office of Program Development, the bulk of the work in her office was focusing on in situ and bioreactor work. EPA is spending approximately $\$ 8$ million a year on work at the equivalent of 6.1 , 6.2 level, and between $\$ 4$ and $\$ 5$ million a year is being spent on biotreatment research at the 6.1 level. CPT Myler indicated that the Department of Defense (DOD) has a 5-year time frame to get things going in the in situ program; he further stated that remediation, rather than study, is the DOD approach, and the DOD wants everything cleaned up in 10 years. Dr. Wilson offered the use of equipment he had purchased and used for 6 months and wondered if it could be shared to help amortize the costs. Dr. Wilson estimated that his program at EPA's RSK Lab uses $\$ 8$ million a year total 6.1, 6.2 funding for long-term basic research, $\$ 5$ millon a year to contractors, and $\$ 1$ millon a year 6.3 funding for field work. 
7. Dr. Alexander returned to the question of the research needs that can be investigated while other field-ready methods are being actively applied. His concern was with the focus of the proposed technical approach. Dr. McCarty indicated that enough has been done in the bioremediation area to get something in the field now, while research is being conducted in the laboratory. $\mathrm{Dr}$. Alexander suggested using available literature to further refine the focus on the compounds to be investigated and that it is important to determine why compounds that are degradable in the laboratory still persist in the field. Dr. McCarty suggested that an easily remediated site be chosen for a field demonstration--an example being a site with soil characteristics that are desirable for the purpose. Dr. Wilson cautioned against using a synthetic soil or groundwater. $\mathrm{Dr}$. Cullinane suggested that the research program focus on one or two sites, one soil type, and one contaminant, depending on what the budget will allow.

8. Mr. Zappi then presented the study approach. The contaminated media WES intends to investigate in the research program include: saturated soils, unsaturated soils, groundwater, spent activated carbon, and gas streams. The groups of contaminants to be included in this study are the chlorinated solvents TCE and methylene chloride, BTEX compounds, petroleum hydrocarbons, methyl ethyl ketone, and the explosives TNT and RDX. Soil types with low and high clay content and high organic content will be evaluated. Sources of soil and water (actual samples versus synthetically formulated), sources of spent activated carbon (laboratory-generated versus samples from operating fullscale systems), and sources of gas streams (air stripped from aqueous solutions versus vapors from pure solutions) will also be evaluated.

9. Dr. Robert Sproull, a visiting Professor from Oregon State University, participating in the WES-sponsored Summer Faculty Research and Engineering Program, addressed the topic of lessons learned. He presented an overview of in situ bioremediation case studies reported in the literature. Dr. Sproull discussed the type of microorganisms present, oxygen sources at sites, delivery systems, and the use of surfactants or other specialty chemicals. There are many successes for bioremediation reported in the literature. However, bioremediation was usually used at small sites with little detail presented and in conjunction with other treatment technologies. Dr. Sproull found no bioremediation of explosives in his case study search. He suggested that future work be directed toward enlisting the help of oil companies and the American Petroleum Institute in obtaining information regarding their research. He also suggested contacting environmental engineering firms about their case studies, including failures.

10. Dr. Alexander again suggested that the research program determine why contaminants persist at a particular site even though the contaminants are quite biodegradable at other sites or in the lab. Dr. Ward pointed out that there are few quantitative data sets in the literature. He feels that this study should be a quantitative demonstration and site selection is very critical. He also cautioned against relying on the data presented in some of the specific case studies mentioned. Documentation is critical for the creditability of the study. He recommends that the study be documented very carefully and mass balances be performed. Dr. Sproull brought up the question of what constitutes a failure. At this point, there was a discussion about what USATHAMA considers a failure and what are steps in the development of technology. The point was made that failure to successfully demonstrate biotreatment would likely adversely affect the Army's image of biotreatment. There was a 
general consensus among the members that getting specific information from private companies about failures of their technologies will be difficult to accomplish. The confidentiality between client and contractor will most likely prohibit obtaining any specific facts.

11. After lunch; Dr. Gunnison and Mr. Zappi presented the proposed saturated soils research. Dr. Gunnison described a tiered screening approach. Tier I involves the verification of contaminant degradation and assessment of a wide variety of treatment conditions to determine which one will support/enhance degradation in pump-and-treat systems. Tier II combines the successful treatment conditions observed in Tier I to optimize treatment. Tier III confirms the optimum treatment condition determined in Tier II using bench-scale column systems. Tier IV involves the evaluation of the optimal treatment condition from Tier III using large scale soil column systems. Dr. Alexander began the discussion of this subject area by suggesting that the screening system should start at Tier III. He indicated that a four-tiered system involves too many steps. WES should focus on determining the limiting factors and why biodegradation does or does not work. He once again indicated that if WES finds that a contaminant is biodegradable, the question to be asked is "Why isn't the contaminant degrading in the field?" He also pointed out that there is a difference between soil and slurry systems. It was his opinion that slurries are too far removed from the real world to be of any use in predicting biodegradation in field situations. Slurries, because they are primed for degradation, may give us a large number of false positives that will only be thrown out at the Tier III level. The environmental problem will be a major factor, and he suggested that during Phase III, a very small column, such as a test tube, be used instead of a large column. Dr. McCarty suggested that the screening process should be done on paper first, before going to the laboratory, and that if a lot of information is known about the contaminant of concern, then the screening process could start at Tier III.

12. Dr. Alexander interjected that characterizing the processes carried out by the organisms present will be of more value than enumerating microorganisms. Essentially, he suggests that WES measure the degradation rate and not population levels. It is not important to know the number of organisms present or which organism is carrying out the degradation; what is important is verifying that degradation is occurring. It will be important to have a laboratory configuration duplicating conditions in the field to determine what prevents organisms from mineralizing the contaminants in the field.

$\mathrm{Dr}$. Wilson indicated agreement with $\mathrm{Dr}$. Alexander. It is also important to be sure that degradation is not being caused by "introduced (contaminating) microorganisms." Dr. Alexander reiterated that it is not necessary to know what organisms are present; monitor the degradation rates and the resulting products, not the number of organisms. He suggested that WES develop a test apparatus that provides the proper environment. For example, if there is only $25 \mathrm{mg}$ of oxygen per gram of soil present, it may be necessary to supply additional oxygen. Dr. Wilson mentioned a column in situ study being conducted by Dr. Gilliam, a Canadian researcher. He indicated that Gilliam was injecting ${ }^{14} \mathrm{C}$ in the field and in columns for evaluating mineralization. Dr. McCarty suggested that the organisms are selected by the biodegradation process that is actively occurring, while $\mathrm{Dr}$. Alexander said that the pathway determination needs to be conducted at the petri dish level. Dr. McCarty also suggested using a dehydrogenase assay in situ to indicate biological activity at the site. Other parameters that can be monitored include tetrazolium dyes, $\mathrm{CO}^{2}$ evolution, and glucose loss in columns. 
13. Mr. Zappi then presented the objectives of pretreatment technologies. The candidate technologies include: (a) chemical oxidation, (b) ultraviolet oxidation, (c) reductive dehalogenation, and (d) KPEG (potassium polyethlyene glycolate). Dr. Alexander mentioned that a denitrogenation system is available. Dr. Spaine brought up the point that mobility through the soil system will be a problem. Then there was a general discussion regarding state regulations that may prohibit the injection of compounds in the ground. Dr. Alexander indicated that abiotic treatment systems are too expensive (e.g., lithium aluminum hydride). He also indicated that ethylene glycol systems (like KPEG) are good above ground, but not for in situ because glycols are too viscous and immobile for circulation.

14. Dr. Judy Pennington presented the proposed approach on the role of sorption and mass-transfer limitations in bioremediation of contaminated saturated soils and groundwater in aquifers. The objectives of this work are to define and quantify these items and minimize adverse effects of sorption processes on in situ bioremediation of saturated soils and groundwater. The proposed approach involves batch and column testing. Batch tests would be used to determine the reversibility of the adsorption/desorption process, sorption kinetics, intraparticle sorption, extraction enhancement, and the relationship between sorption and oil properties. The column tests would be used to validate batch-based concepts and determine film and immobile water effects, operational strategies, and extraction enhancement.

15. Following this, Dr. Pennington considered the role of sorption and mass transfer in bioremediation of unsaturated soils. The objectives for unsaturated soils and saturated soils are the same, with the added objective of evaluating the significance of the gas phase in the vadose zone.

16. Discussion on sorption in saturated soils was initiated by Dr. Alexander who questioned whether the word "sorption" referred to adsorption or desorption. His main concern, however, was whether or not sorption is a limiting factor. Some contaminants may be unavilable in the sorbed state, while some recent research suggests that microorganisms may be able to use the compound in the sorbed state. CPT Myler asked whether hydraulic desorption would be used to decrease the treatment time. The Working Group members were in agreement that it is difficult to release adsorbed contaminants from natural site sediments, meaning that because of the kinetics of contaminant desorption, the cleanup of a site could take years. Dr. Alexander's opinion was that getting the contaminant of interest into solution should not be a big concern. A discussion then followed on the use of surfactants. It was brought out that the University of Michigan is doing research on surfactants at the present time. The Institute for Gas Technology (Chicago, IL) is also conducting research in this area and has organisms able to produce their own surfactants. A discussion followed on the merits of using surfactants in the environment. The Working Group seemed to be fairly universal in their agreement that sorption is a major issue that should be addressed with research. However, they also generally agreed that adding surfactants to the environment for the purpose of removing contaminants from soils does not seem feasible. The concern is that a large volume of surfactant will be required to remove the sorbed contaminants from the soil. The resulting demand for nutrients, oxygen, etc., required to degrade the surfactant will outweigh all the advantages obtained by using the surfactant. 
17. Dr. Spaine initiated a discussion on unsaturated soils by stating that the sorption work should concentrate on RDX and TNT, because the other compounds are water soluble. Mr. Wayne Sisk indicated that concentrations of explosive contaminants are so high in the soil that there may be aggregates of contaminants present. Mr. Zappi suggested that where this is true, the upper layers of soil should be scraped off and incinerated. Bioremediation can then be applied to the residuals in the soil. Then Mr. Sisk indicated that we need to bioremediate everything that is present, even the large lumps of TNT. A discussion then followed on the limitations of bioremediation and the most appropriate location to conduct bioremediation. Dr. McCarty suggested that the evaluation should be conducted at the site. He cited a recent study where it was found that TCE was sorbed more on the surface of sand than would be expected and little TCE was found in the solution phase.

18. Dr. Stafford Cooper of the Geotechnical Laboratory presented the Tri Services Cone Penetrometer research and development efforts currently being conducted at WES. Mr. Zappi discussed the potential of the cone penetrometer to be used as an injection system for the addition of oxygen sources, cometabolites, nutrients, and inoculum into soil or aquifers and for collection of water and soil samples. The advantages of the cone penetrometer are significantly reduced installation costs, reduced effects of biofouling, and in situ contaminant detection. Dr. Wilson's concern was a monetary one. He questioned the cost factor between drilling wells and the cone penetrometer. He also stated that a cone penetrometer is a soil tool and cannot be used where the ground consists of boulders or stone. There followed a general discussion concerning the type of grout that would be used to fill the injection ports. Also of concern was the cone penetrometer's pipe casing, which cannot be left in place for subsequent application of nutrients, etc. The pressure around the pipe casing exerts a force which makes the extraction of the pipe impossible. Dr. Wilson did not feel that the cone penetrometer was a feasible injection method, but that it could be useful in the detection of contaminant plumes.

19. After the discussions concluded, the Working Group members were given a tour of the analytical laboratory, the Ecosystem Research and Simulation Division laboratories, and the Hazardous Waste Research and Development Center.

20. The working group meeting continued on 15 August 1990. Dr. Shanka Banerji, Professor of Civil Engineering at the University of MissouriColumbia, also participating in the Summer Faculty Research and Engineering Program, and Mr. Zappi presented the work to be conducted on the area of bioregeneration of spent activated carbon. The objective of this research topic is to determine the feasibility of regeneration of spent carbon. The focus is to first evaluate the regeneration of carbon contaminated with phenol as a model compound and then determine regeneration of carbon contaminated with the target compounds. Several application scenarios are to be evaluated including (a) in-bed regeneration, (b) slurry reactor regeneration, (c) fluidized column regeneration, and (d) in-bed contaminant extraction.

21. Discussion on the carbon regeneration work was initiated by Dr. McCarty, who spoke of the work being conducted in Sweden. His suggestion was that a combination of regeneration and adsorption be conducted. Dr. Alexander cited the example of diisopropyl methylphosphonate (DIMP), a contaminant of concern at Rocky Mountain Arsenal. There is no reason for organisms to utilize DIMP when phenol is present because DIMP must be degraded via cometabolism since it 
cannot be used as a carbon source. Dr. Alexander stated that it is not necessary to desorb the compounds, and that if given a chance, the population of organisms will be sufficient to physically come into contact with the contaminant. Also, he suggested that cometabolism be used to initiate degradation of the contaminants. He gave the example of using mono and dinitro toluenes and benzene to develop populations of microorganisms active against TNT and RDX. He indicated that quite a bit of time may be required to obtain active populations. Dr. McCarty considered the use of simple compounds to stimulate degradation. He referenced current work at his laboratory where small columns with activated carbon and dihalogenated benzenes at trace levels yielded rapid removal of contaminants at dilute levels. He also mentioned use of trace levels of acetate $(1 \mathrm{mg} / \ell)$ to support active degradation requiring very short detention times. Dr. Wilson suggested the regeneration alternate between an anaerobic and aerobic mode by occasionally passing several pore volumes of aerated clean oxygenated water through the columns.

22. Mr. Zappi began an additional discussion on the details of regeneration by asking the group how they would suggest removing microorganisms from the carbon once regeneration has been completed (with regard to the contaminant) and how they would control the particle size of the regenerated carbon. CPT Myler suggested using toluene, rather than phenol, as a model compound for carbon regeneration since toluene is a real problem for the Army. Dr. Alexander indicated that the phosphonate, as in DIMP, is a problem. Microorganisms cannot disrupt the carbon-phosphorus bond in phosphonate when phosphonate is present, because phosphate inhibits phosphonate cleavage. Dr. McCarty mentioned that the water to be used in the columns might be pretreated to remove compounds from the water that might support growth of populations of biofouling organisms. He gave the example of using biological pretreatment to remove acetone before use of air stripping to remove TCE. He further suggested that in looking at sorption and degradation, an ideal situation is to use carbon plus a fixed bed with dilute waste. CPT Myler also asked about the desirability of encouraging the growth of thermophiles (up to $55^{\circ} \mathrm{C}$ ). Dr. Alexander indicated that this would decrease the species diversity (of the degrading microorganisms), but that it might be worth attempting. Dr. Wilson mentioned that the concern CPT Myler expressed is really a third or fourth order question. It is really too early to get into such novel ideas. Dr. Alexander suggested that WES look for organisms active on sorbed compounds. He asked which is faster in soils--the rate of mineralization or the rate of desorption. Then he indicated that the rate of mineralization would probably be faster.

23. Mr. Zappi and Dr. Gunnison presented the proposed approach for evaluating biotreatment of unsaturated soils. This approach follows the same treatment approach presented on the saturated soils. Two systems are being considered for the Tier III Level - an annular ring system and a column system. The requirements for the system are: (a) adjustable water content, (b) adjustable air flow rate, (c) nutrient injection, and (d) inoculum injection. The Working Group questioned the residence time and water gradient. The Working Group also questioned how this system could be applied to the field in an effective way. Dr. Alexander suggested that a soil physicist be consulted when the Tier III units are being set up. Dr. Wilson felt that measuring the matrix potential is of more value than measuring the water content, because the air and water in the field can be measured by the matrix potential. 
24. Dr. Gunnison presented the proposed work for the vapor phase research. The vapor phase in microbiology of biotreatment systems includes the development of a cometabolic population and the design and construction of benchscale biofilters. Dr. Wilson cited work being conducted by Dr. Barbara Wilson, EPA RSK lab. She is currently using cometabolic populations developed on propane and/or-butane in air to treat TCE vapor and getting 95 percent removal in $15 \mathrm{~min}$. She is also working at the pilot scale wherein cometabolic populations supported on celite or diatomaceous earth are capable of removing 65 to 75 percent of TCE at a concentration of $300 \mu \mathrm{g} / \ell$ in 30 to $40 \mathrm{~min}$. Dennis Miller and Don Kampbe11, associates of $\mathrm{Dr}$. B. Wilson, are able to achieve 95 to 98 percent removal of TCE in one pass with cometabolic populations supported on $2 \mathrm{mg} / \ell$ butane. Dr. Gunnison asked whether the units would develop statisfactory cometabolic populations if the filters were transported to the field and set up, or was inoculation required? Dr. Wilson stated that the existing system would be too large to practically treat the flow rate from an air stripping unit. A discussion followed on the merits of using propane rather than methane to support development of cometabolic populations. Propane was found to be better at Ada, OK, but not at a site in California. Therefore, inoculation of the filters may be required to develop suitable populations on propane in the field. In addition, propane supports the growth of Nocardia and other actinomycetes, as opposed to varieties of the bacterium Pseudomonas supported on methane. Actinomycetes are more desirable than bacteria for use in vapor treatment, particularly where the treatment system is likely to be shut down periodically. The actinos resist desiccation, whereas the bacteria are very susceptible to it. Brian Folsom's work on vapor phase systems at EPA Gulf Breeze was mentioned.

25. Mr. Zappi then presented proposed work on use of colloidal gas aphrons (CGAs) and electrokinetics (EK). Colloidal gas amphrons are small microbubbles ( 5 to $10 \mu \mathrm{m}$ in diameter) that have the potential to be used as an oxygen source for microbial populations and to transport additives through soils having low hydraulic conductivities. Electrokinetics is a process in which water and small colloids may be transported through porous media at velocities much faster than is achievable using advective forces. Colloidal gas aphrons can be used as an oxygen source for microbes in biologically active zones and as a means of transporting nutrients, microbial inocula, alternate electron acceptors, and surfactants. Mr. Zappi also discussed the desirability of producing CGAs containing oxygen and methane to support cometabolic populations degrading halogenated alkanes. Electrokinetics has potential use for inducing transport of CGAs, transporting additives through soil, transporting inocula through soils, controlling the velocity and direction of groundwater flow, and flushing the soil of contaminants for aboveground treatment. $\mathrm{Dr}$. Ward questioned the ability of microorganisms to get to the CGAs. $\mathrm{Dr}$. Alexander mentioned that the mobility of microorganisms in an electric field is species specific. Therefore, electrokinetics is likely to work for some species, but not for others. Dr. McCarty indicated that investigation of CGAs should start with a field level effort.

26. The proposed work on the fate of additives in soil systems was presented by Mr. Zappi. Among the additives discussed were oxygenated water, hydrogen peroxide, air sparging, and surfactants. The objective of the work on additives is to determine the effect of additives on hydraulic conductivity and to assess the fate of additives. The experimental approach will involve use of permeameter and column testing. At Traverse City, MI, investigators have been able to inject $20 \mathrm{mg} / \ell \mathrm{O}_{2}$ without producing bubbles in fine material, and the 
oxygen has moved up to 50 to $60 \mathrm{ft}$ from the injection site in porous medium. A brief discussion on the merits of using hydrogen peroxide then followed. $\mathrm{H}_{2} \mathrm{O}_{2}$ is toxic to many organisms, particularly those lacking catalase (an $\mathrm{H}_{2} \mathrm{O}_{2}$ decomposing enzyme); and, therefore, peroxide is not always desirable. CPT Myler mentioned studies conducted at Eglin Air Force Base and Virginia Polytechnic Institute. Dr. Spaine indicated that the report on Eglin should be interpreted with caution. Dr. Wilson suggested that polyphosphate be used as a stabilizer for peroxide. There was a short discussion on whether polyphosphate acts as a catalyst, chelator, or just complexes with contaminants.

27. Another discussion followed on the merits of using surfactants to desorb contaminants from soil surfaces. Dr. Alexander indicated that cationic surfactants are undesirable because they are toxic and tend to sorb to soil systems. Dr. Ward indicated that Mr. Zappi's list of additives is long, and use of additives requires a lot of basic laboratory research, 3 to 5 years for each.

28. A group picture (Appendix B) was taken on the steps outside the Environmental Laboratory, after which the group left for lunch. Drs. Ward, Wilson, and Spaine were unable to return after lunch because of their respective flight schedules.

29. Mr. Zappi presented the task objectives for an artificial aquifer system. An artificial aquifer system will be developed as an experimental apparatus that can be used to evaluate in situ treatment technologies on a large scale, but under controlled conditions. The system will be approximately 50 to $70 \mathrm{ft}$ in length and a width greater than $5 \mathrm{ft}$; the depth will be at least $5 \mathrm{ft}$, and the hydraulic gradient will be adjustable via a change in pumping rate and an adjustable slope. The system will be described using a numerical model. The aquifer material can be changed, and there will be rainfall simulation for vadose zone modeling. The system will be able to investigate the effects of aquifer heterogeneity in a controlled 3-D system of appreciable size.

30. A lengthy discussion then ensued regarding the advisability of using an artificial aquifer. Dr. McCarty mentioned work being conducted by Garrison Sposito at Berkley. He advised that the objectives for use of an artificial aquifer system need to be clear. He indicated that WES needs to be aware of the artificial aquifer's limitations, including destruction of the soil structure and permeability, effects on distribution of organisms, and paths for water. Dr. Alexander asked "What is the virtue of going to something that large that also lacks the structure of the field?" He feels a good core system may be more representative of the natural site. Dr. McCarty further indicated that dispersion, sorption, and microbiology are not the same as in the field, where there are cracks, roots, etc. Dr. Alexander continued by suggesting the use of experimental units smaller than the artificial aquifer. For example, experimental units consisting of $10 \mathrm{qt}$ of soil are better than one large batch. Even 10 qt won't work well because the physical characteristics of the field are variable. Bringing large blocks of the actual soil of concern to the lab is more feasible than using a 60 - by 6 - by 5 -ft artificial aquifer. The initial reaction to the artificial aquifer is no. Also, rather than using actual contaminants in the field, WES should use simulants (compounds that represent the contaminant, but are not noxious to the environment) --i.e., permitted pesticides or unpermitted, 2,4-D for toluene or esters of 2,4-D for toluene. Dr. Alexander suggested that WES talk with soil physicists or others who have tried this, such as the people at Riverside and/or Davis, 
to develop an understanding of what disruption of soil structure does to soil properties--Bill Drury's work at Riverside and Sposito's efforts at Berkley. Dr. Alexander stressed that the importance of field-scale evaluation should also be examined. For example, inoculation of soil with microorganisms rarely works in the field because of physical and environmental considerations. Dr. McCarty referenced work done at Bordon Air Force Base in Canada on sorption, degradation, and dispersion.

31. A slide was presented by Mr. Zappi describing two physical models built to verify the HELP computer model developed by Dr. Paul Schroder at WES. Mr. Myers stated that the HELP computer model was used for a land disposal study where the fill material was known. It is considered doubtful that the Help computer model would be capable of predicting the contaminant flow in a disturbed, unknown soil matrix. Once a soil sample is physically disturbed, the natural integrity of the sample is destroyed and cannot be used to replicate a natural environment. Dr. Alexander stated that research should be done on a small scale in the field, with some testing in the laboratory. He suggests finding a site where success will be high and using simulants instead of target contaminants in the field to simulate the effects of the contaminants of concern. He also feels that an artificial aquifer would be useful in $a{ }^{14} \mathrm{C}$ labeled study.

32. Mr. Zappi then asked for a closing discussion and comments from the Working Group. Both Dr. Alexander and Dr. McCarty agreed the overall general approach was good, but that the scope of the work was too large. Dr. Alexander stated that his major concern was the lack of chemistry in the draft approach document they had received. Dr. McCarty indicated that the study needed extensive work on focusing the objectives, especially on what is applied and what is research. Dr. McCarty suggested that more focus on the chemistry of the Army compounds of interest be included in the study. Both Dr. Alexander and Dr. McCarty agreed that WES needed a senior analytical chemist on its in situ biotreatment staff.

33. Mr. Sisk stated that the study will be prioritized on the amount of money available. The budget is not known yet for next year or for the total project. The approach will not be fully funded and must be prioritized based on available funds.

34. Mr. Zappi concluded by thanking the Working Group for attending the meeting and sharing their expertise. There was some discussion about a possible future meeting. Future meetings of the technical Working Group will be scheduled as results of the research program become available.

Cynthia Teeter

Physical Scientist 
Public reporting burden for this collection of information is estimated to average 1 hour per response, including the time for reviewing instructions, searching existing data sources. gathering and maintaining the data needed, and completing and reviewing the collection of information. Send comments Dovis Highway, Suite 1204. Arlington, VA 22202-4302, and to the Office of Management and Budget, Paperwork Reduction Project (0704-0 188), Washington, DC 20503.

\begin{tabular}{|l|c|c|}
\hline 1. AGENCY USE ONLY (Leave blank) & $\begin{array}{c}\text { 2. REPORT DATE } \\
\text { August } 1993\end{array}$ & $\begin{array}{c}\text { 3. REPORT TYPE AND DATES COVERED } \\
\text { Final report }\end{array}$ \\
\hline
\end{tabular}

\section{TITLE AND SUBTITLE}

5. FUNDING NUMBERS

Technical Approach for In Situ Biological Treatment

Research: Bench-Scale Experiments

\section{AUTHOR(5)}

Mark E. Zappi, Douglas Gunnison, Judith Pennington, M. John Cullinane, Cynthia L. Teeter, James M. Brannon, Tommy E. Myers, Shankha Banerji, Robert Sproull

\section{PERFORMING ORGANIZATION NAME(S) AND ADDRESS(ES)}

US Army Engineer Waterways Experiment Station Environmental Laboratory, 3909 Halls Ferry Road Vicksburg, MS 39180-6199

8. PERFORMING ORGANIZATION REPORT NUMBER

Technical Report IRRP - 93 - 3

10. SPONSORING/MONITORING AGENCY REPORT NUMBER

US Army Corps of Engineers

Washington, DC 20314-1000

\section{SUPPLEMENTARY NOTES}

Available from National Technical Information Service, 5285 Port Royal Road, Springfield, VA 22161.

12a. DISTRIBUTION/AVAILABILITY STATEMENT

12b. DISTRIBUTION CODE

Approved for public release; distribution

is unlimited

\section{ABSTRACT (Maximum 200 words)}

The US Army Engineer Waterways Experiment Station has assessed the potential of in situ biotreatment for remediation of contaminated aquifers. The objectives of this effort were to determine the knowledge gaps associated with in situ biotreatment, identify knowledge gaps in applications methodology, and to develop a research program that will improve the field applicability of the technology. The assessment involved review of pertinent literature, discussions with key authorities in the field, interviews with individuals associated with companies having experience with the application of in situ biotreatment at contaminated sites, and review of 27 case studies.

A description of pertinent design components required for proper implementation is presented. The successes and failures of past attempts at implementing in situ biotreatment are discussed. Finally, a bench-scale research approach is presented that will bridge many of the knowledge gaps identified. This approach will result in conversion of in situ biotreatment to a viable remediation technology applicable to numerous contaminated military sites.

\begin{tabular}{|c|c|c|c|}
\hline \multicolumn{3}{|l|}{ 14. SUBJECT TERMS } & 15. NUMBER OF PAGES \\
\hline $\begin{array}{l}\text { Bench studies } \\
\text { In situ biotreatment }\end{array}$ & \multicolumn{2}{|c|}{$\begin{array}{l}\text { Microbes } \\
\text { Site remediation }\end{array}$} & $\frac{231}{\text { 16. PRICE CODE }}$ \\
\hline $\begin{array}{l}\text { 17. SECURITY CLASSIFICATION } \\
\text { OF REPORT }\end{array}$ & $\begin{array}{l}\text { 18. SECURITY CLASSIFICATION } \\
\text { OF THIS PAGE }\end{array}$ & $\begin{array}{l}\text { 19. SECURITY CLASSIFICATION } \\
\text { OF ABSTRACT }\end{array}$ & 20. LIMITATION OF ABSTRACT \\
\hline UNCLASS IFIED & UNCLASSIFIED & & \\
\hline
\end{tabular}

